\title{
DAS WECHSELVERHALTEN VON KONSUMENTEN IM STROMMARKT
}

\section{Eine empirische Untersuchung direkter und moderierender Effekte}

\author{
Jörg Henseler \\ Kleve
}

Inauguraldissertation

zur Erlangung der Doktorwürde

(Doctor rerum politicarum)

am Fachbereich Wirtschaftswissenschaften der

Technischen Universität Kaiserslautern 

Die Veröffentlichung dieser Dissertation erfolgt im

Deutschen Universitätsverlag (DUV), Wiesbaden. 
Diese Seite ist für den Schmutztitel reserviert. 
Für Katrin 


\section{Geleitwort}

Ende der 1990-er Jahre wurde der deutsche Strommarkt liberalisiert. Seitdem konkurrieren Stromanbieter um Privatkunden. Stromanbieter, die am Markt bestehen wollen, müssen die Fähigkeit entwickeln, Kunden zu gewinnen und zu binden. Hierzu ist eine profunde Kenntnis des Konsumentenverhaltens notwendig. Allerdings gibt es bisher weder in der Marketingwissenschaft noch in der Marketingpraxis schlüssige Modelle, die das Wechselverhalten von Konsumenten zu erklären vermögen. Hier setzt die vorliegende Arbeit an: Erstmalig wird das Wechselverhalten von Konsumenten im Strommarkt modelliert und analysiert.

Ausgehend von wissenschaftlichen Untersuchungen in vergleichbaren Märkten sowie konzeptionellen Überlegungen modelliert der Autor vier Determinanten des Wechselverhaltens:

- die Kundenzufriedenheit mit seinem bestehenden Anbieter, d. h. inwieweit die Erwartungen an den Stromanbieter erfüllt werden,

- die Attraktivität der Alternativen, d.h. wieviel Nutzenzugewinn sich durch einen Anbieterwechsel erzielen ließe,

- die wahrgenommenen Wechselkosten, d.h. die Konsumenteneinschätzung der mit einem Anbieterwechsel verbundenen Mühen und Risiken, sowie

- das Involvement, d.h. der Grad des sich Interessierens für und sich Befassens mit der Stromversorgung.

Aus bestehenden Theorien wie bspw. der von Thibaut und Kelley (1959) entwickelten Austauschtheorie leitet der Autor Hypothesen zu direkten und moderierenden Effekten dieser Konstrukte auf das Wechselverhalten von Konsumenten im Strommarkt ab. Das so generierte konzeptionelle Modell wird einer empirischen Überprüfung unterzogen.

Die vorliegende Arbeit leistet neue und wesentliche Beiträge zum wissenschaftlichen Fortschritt in der Marketingforschung. Sie bietet eine Erweiterung des Wissensstands zum Wechselverhalten von Konsumenten in Versorgungsbeziehungen. Sie enthält darüber hinaus eine ausführliche Abhandlung zur Strukturmodellierung und -analyse mit der Methode der PLS-Pfadmodellierung, die dem empirischen Teil der Arbeit zugrunde liegt. 
Die Interpretation der empirischen Befunde für die praktische Marketingtätigkeit ist schlüssig und Nutzen stiftend: Energieversorgungsunternehmen erhalten Empfehlungen, wie sie Anreize zum Verbleiben bzw. Wechsel von Privatkunden geben können: Unternehmen in der Verteidigerposition, wie sie das Wechseln der eigenen Kunden reduzieren; Unternehmen in der Angreiferposition, wie sie das Wechseln von Konkurrenzkunden fördern können. Auch für andere Institutionen, die den Strommarkt direkt oder indirekt beeinflussen, so wie Gesetzgeber, Regulierer oder Fachverbände, sind die in dieser Arbeit gewonnenen Erkenntnisse von Nutzen. Dieses Buch ist nicht nur für Wissenschaftler, sondern besonders auch für Praktiker ein Gewinn.

Prof. Dr. Friedhelm W. Bliemel 


\section{Vorwort}

Die Liberalisierung des Strommarkts Ende der 1990-er Jahre bescherte Konsumenten erstmals die Möglichkeit, zwischen verschiedenen Stromanbietern zu wechseln. Neue Stromanbieter sahen hierdurch die Chance, Stromkunden zu gewinnen, und etablierte Stromanbieter sahen die Notwendigkeit, ihren Kundenstamm zu verteidigen. Um im Markt Erfolg zu haben, benötigen Stromanbieter Wissen über das Wechselverhalten von Konsumenten. Bislang verfügen jedoch weder Marketingwissenschaft noch -praxis ausreichend über dieses Wissen. Hinzu kommt, dass Meinungsumfragen zwar teilweise eine Wechselbereitschaft von mehr als 30 Prozent der deutschen Haushalte ermittelten, jedoch weniger als fünf Prozent der Haushalte ihre Wechselabsicht in die Tat umsetzten. Dies lässt die folgenden beiden Fragen in den Vordergrund treten: Welche Faktoren beeinflussen das Wechselverhalten von Konsumenten im Strommarkt? Warum unterscheidet sich die bekundete Wechselabsicht in so großem Maße vom realisierten Wechselverhalten?

Diese Fragen bildeten den Ausgangspunkt der vorliegenden Arbeit, die im Dezember 2005 vom Fachbereich Wirtschaftswissenschaften der Technischen Universität Kaiserslautern als Dissertation genehmigt wurde. Diese Arbeit entstand während meiner Zeit als wissenschaftlicher Mitarbeiter am Lehrstuhl für Betriebswirtschaftslehre insbesondere Marketing der Technischen Universität Kaiserslautern sowie zu Beginn meiner Zeit als Universitair Docent an der Faculteit der Managementwetenschappen, Radboud Universiteit Nijmegen, Niederlande. Großer Dank gebührt meinem akademischen Lehrer Prof. Dr. Friedhelm W. Bliemel für die Betreuung dieser Arbeit sowie die vertrauensvolle Zusammenarbeit während meiner Zeit als Doktorand und Mitarbeiter an seinem Lehrstuhl. Auch gilt ihm mein Dank für die konstruktive Kritik und die wertvollen Hinweise sowie für den Freiraum, den er mir bei der Bearbeitung des Themas und meiner übrigen Tätigkeiten am Lehrstuhl ließ. Schließlich möchte ich ihm für die erfahrungs- und lehrreiche Zeit danken. Was ich von ihm lernte, ging weit über das Akademische hinaus. Weitere wertvolle Rückmeldungen erhielt ich von der Faculty und den Teilnehmern des $17^{\text {th }}$ EMAC Colloquium for Doctoral Students in Marketing. Für die Übernahme des Zweitgutachtens, aber auch die wissenschaftliche Zusammenarbeit, danke ich Herrn Prof. Dr. Volker Lingnau. Mein Dank gilt weiterhin Herrn Prof. (em.) Dr. Heiner Müller-Merbach als Fachprüfer im Promotionsverfahren sowie Herrn Prof. Dr. Reinhold Hölscher als Vorsitzendem der Promotionskommission. 
Für die tiefgehenden Diskussionen und die hervorragende Zusammenarbeit möchte ich meinen Kollegen Herrn Dr. Kai Adolphs, Frau Prof. Dr. José M. M. Bloemer, Herrn Universitätsdozent Dr. Paul H. Driessen, Herrn Prof. Dr. Andreas Eggert, Herrn Privatdozent Dr. Georg Fassott, Herrn Hauptdozent Dr. Bas Hillebrand und Herrn Prof. Dr. Edwin J. Nijssen danken. Für die kritische Durchsicht des Manuskripts danke ich Frau Stefanie Jung und Frau OStR Rosemarie García Lozano. Für die Assistenz bei der Literaturbeschaffung und -administration und für die Erstellung von Grafiken und Tabellen sei Herrn cand. Wirtsch.-Ing. Jochen Karls, Frau cand. math. tech. Zahra Lakdawala, Herrn cand. Wirtsch.-Ing. Christian Schröder sowie Frau cand. Wirtsch.-Ing. Sonja Worm mein Dank ausgesprochen. Den Teilnehmern des Faches „Marktforschung und Marketinginformationssysteme“ im Wintersemester 2003/04 danke ich für die tatkräftige Mitwirkung an der Datenerhebung.

Ganz besonderer Dank gebührt meinen Eltern für den Rückhalt und die Unterstützung, die sie mir während meiner wissenschaftlichen Ausbildung zukommen ließen. Schließlich möchte ich an dieser Stelle ganz herzlich meiner Frau Katrin García Boy danken, die mich in der schwierigen Zeit der Erstellung dieser Arbeit mit ganzer Kraft unterstützt hat. Ihr widme ich diese Arbeit.

Universitätsdozent Dr. Jörg Henseler 


\section{Inhaltsverzeichnis}

Geleitwort vii

Vorwort $\quad$ ix

Tabellenverzeichnis $\quad$ Xv

Abbildungsverzeichnis $\quad$ xvii

Abkürzungsverzeichnis $\quad$ xix

Symbolverzeichnis $\quad$ xxi

1 Einführung 1

1.1 Relevanz des Themas . . . . . . . . . . . . . . . . . . . . . 3

1.2 Problemstellung . . . . . . . . . . . . . . . . . . . . . 5

1.3 Aufbau der Arbeit . . . . . . . . . . . . . . . . . . . 6

2 Untersuchungsobjekt und bestehende Erklärungsansätze 9

2.1 Der Strommarkt für Haushaltsstromkunden . . . . . . . . . . . . . . . . . 9

2.2 Wechselverhalten bei Versorgungsbeziehungen . . . . . . . . . . . . . 14

2.2.1 Versorgungsbeziehungen . . . . . . . . . . . . . 15

2.2.2 Empirische Studien mit explorativem Charakter zu Versorgungsbeziehungen . . . . . . . . . . . . . . . . . 18

2.2.3 Empirische Studien mit konfirmatorischem Charakter zu Versorgungsbeziehungen . . . . . . . . . . . . . . . . . 20

2.3 Ursachen für die Diskrepanz zwischen Wechselabsicht und -vollzug . . . . . 23

2.4 Sind Konsumenten zu träge, um den Anbieter zu wechseln? . . . . . . . . . 27

3 Theoretische Grundlagen $\quad 31$

3.1 Theorien zur Fortsetzung von Versorgungsbeziehungen . . . . . . . . . . . 31

3.1.1 Die Lerntheorie der instrumentellen Konditionierung . . . . . . . . 31

3.1.2 Wille vs. Zwang zur Fortsetzung einer Geschäftsbeziehung . . . . . 32 
3.2 Theorien zum Anbieterwechsel bei Versorgungsbeziehungen . . . . . . . . . 34

3.2.1 Die Theorie der kognitiven Dissonanz . . . . . . . . . . . . . . . . 35

3.2.2 Die Theorie überlegten Handelns . . . . . . . . . . . . . . 36

3.2.3 Die Theorie des geplanten Verhaltens . . . . . . . . . . . . 38

3.2.4 Die Austauschtheorie . . . . . . . . . . . . . . . . . . . . . . . . 40

4 Determinanten des Wechselverhaltens 45

4.1 Kundenzufriedenheit als Determinante des Wechselverhaltens . . . . . . . . 46

4.1.1 Das Konstrukt Kundenzufriedenheit ． . . . . . . . . . . . . . . . 47

4.1.2 Hypothesen zum Einfluss der Kundenzufriedenheit bei Versorgungsbeziehungen . . . . . . . . . . . . . . 53

4.2 Attraktivität der Alternativen als Determinante des Wechselverhaltens . . 55

4.2.1 Konzeptualisierung der Attraktivität der Alternativen . . . . . . . . 56

4.2.2 Hypothesen zum Einfluss der Attraktivität der Alternativen bei Versorgungsbeziehungen . . . . . . . . . . . . . 58

4.2.3 Attraktivität der Alternativen als Moderator der Wirkung der Kundenzufriedenheit . . . . . . . . . . . . . . 60

4.3 Wechselkosten als Determinante des Wechselverhaltens . . . . . . . . . . 61

4.3.1 Wechselkosten vs. Wechselbarrieren . . . . . . . . . . . . . . . 62

4.3.2 Arten von Wechselkosten . . . . . . . . . . . . . . . . . . . 65

4.3.3 Hypothesen zum Einfluss der Wechselkosten bei Versorgungsbeziehungen . . . . . . . . . . . . . . . . . . 71

4.3.4 Wechselkosten als Moderator der Wirkung der Kundenzufriedenheit 72

4.4 Involvement als Determinante des Wechselverhaltens . . . . . . . . . . . . 74

4.4.1 Forschungslinien und Definition . . . . . . . . . . . . 76

4.4.2 Hypothesen zum direkten Einfluss des Involvements innerhalb des Wechselverhaltens . . . . . . . . . . . . . . . . 80

4.4.3 Hypothesen zu Interaktionseffekten des Involvements innerhalb des Wechselverhaltens . . . . . . . . . . . . . . . . . 82

4.5 Modell des Wechselverhaltens . . . . . . . . . . . . . . . . . . . 86

5 Strukturgleichungsmodellierung mit latenten Variablen $\quad 91$

5.1 Aufbau von Strukturgleichungsmodellen . . . . . . . . . . . . . . . . 92

5.1 .1 Strukturmodell . . . . . . . . . . . . . . . . . . . . 93

5.1 .2 Messmodell ... . . . . . . . . . . . . . . . . . . 94

5.1.3 Modellierung von Konstrukten zweiter Ordnung . . . . . . . . . . . 99

5.1.4 Modellierung von moderierenden Effekten . . . . . . . . . . . . . . 101

5.2 Der Partial-Least-Squares-Ansatz zur Modellschätzung . . . . . . . . . . . 102 
5.2.1 Entwicklung der PLS-Pfadmodellierung . . . . . . . . . . . . . . 102

5.2.2 Schätzung von Modellen mit zwei latenten Variablen . . . . . . . . 104

5.2.3 Schätzung von Modellen mit mehreren latenten Variablen . . . . . . 104

5.2.4 Schätzung von Modellen mit Konstrukten zweiter Ordnung . . . . . 109

5.2.5 Schätzung von Modellen mit moderierenden Effekten . . . . . . . . 110

5.3 Beurteilung und Validierung von PLS-Pfadmodellen . . . . . . . . . . . . . 113

5.3.1 Bestimmtheitsmaß und Effektgröße . . . . . . . . . . . . 113

5.3.2 Pfadkoeffizienten . . . . . . . . . . . . . . . . . . . . 114

5.3.3 Bootstrapping . . . . . . . . . . . . . . . . . 115

5.4 Evaluierung und Validierung von Messmodellen . . . . . . . . . . . . 115

5.4.1 Überprüfung der Reliabilität von Messmodellen . . . . . . . . . . . 116

5.4 .2 Überprüfung der Validität von Messmodellen . . . . . . . . . . . . . 118

6 Empirische Untersuchung des Wechselverhaltens von Konsumenten 123

6.1 Ziel der Untersuchung und Abgrenzung des Untersuchungsobjekts . . . . . 123

6.2 Erhebungsdesign . . . . . . . . . . . . . . . . . . . . . . 124

6.3 Operationalisierung der Konstrukte . . . . . . . . . . . . . . . 125

6.3.1 Operationalisierung der Kundenzufriedenheit . . . . . . . . . 126

6.3.2 Operationalisierung der Attraktivität der Alternativen . . . . . . . 129

6.3.3 Operationalisierung der Wechselkosten . . . . . . . . . . . . 131

6.3.4 Operationalisierung des Involvements . . . . . . . . . . . . . 135

6.3.5 Operationalisierung der endogenen Variablen . . . . . . . . . . . . 136

6.4 Beurteilung des Messmodells . . . . . . . . . . . . . . . . . . . . 137

6.4.1 Beurteilung der Indikatorladungen . . . . . . . . . . . . . . . . 138

6.4 .2 Beurteilung der Indikatorgewichte . . . . . . . . . . . . . . 138

6.4.3 Beurteilung der Reliabilität . . . . . . . . . . . . . . . . . . . 140

6.4.4 Beurteilung der Validität . . . . . . . . . . . . . . . . . . . . . . . 142

6.5 Beurteilung des Strukturmodells . . . . . . . . . . . . . . . . . . . . . 144

6.5.1 Beurteilung der Modellgüte . . . . . . . . . . . . . . . . 144

6.5.2 Beurteilung der direkten Effekte . . . . . . . . . . . . . . 147

6.5.3 Beurteilung der Interaktionseffekte . . . . . . . . . . . . . . . . . . 149

6.6 Differenzen der Parameter von Wechselabsicht und Wechselvollzug . . . . . 154

7 Schlussbetrachtung $\quad 159$

7.1 Implikationen für die Marketingwissenschaft . . . . . . . . . . . . . . . . . 159

7.2 Implikationen für die Marketingpraxis . . . . . . . . . . . . . . . . . . . . . 162

7.2.1 Implikationen für Unternehmen in der Verteidigerposition . . . . . . 163

7.2.2 Implikationen für Unternehmen in der Angreiferposition . . . . . . 167 
7.3 Grenzen der Arbeit und Anknüpfungspunkte für weitere Forschung . . . . 170

8 Zusammenfassung $\quad 175$

$\begin{array}{ll}\text { Literaturverzeichnis } & 179\end{array}$

$\begin{array}{ll}\text { Anhang } & 207\end{array}$

A.1 Fragebogen . . . . . . . . . . . . . . . . 207

A.2 Korrelationsmatrix . . . . . . . . . . . . . . . . 211

A.3 Textquellen zur Trägheit . . . . . . . . . . . . . . . . . . . 213

A.3.1 Einfluss von Trägheit aus Sicht etablierter Anbieter . . . . . . . . . 213

A.3.2 Einfluss von Trägheit aus Sicht neuer Anbieter . . . . . . . . . . . . 213

A.3.3 Einfluss von Trägheit aus Verbrauchersicht . . . . . . . . . . . . . . 214 


\section{Tabellenverzeichnis}

2.1 Auswirkungen der Deregulierung der Energiewirtschaft . . . . . . . . . . . 11

2.2 Arten von Gütern und Austauschbeziehungen . . . . . . . . . . . . . 17

3.1 Warum Kunden ihre Geschäftsbeziehung fortsetzen . . . . . . . . . . . . 34

4.1 Vergleich der Konzepte Dienstleistungsqualität und Kundenzufriedenheit . 50

4.2 Vergleich der Sicht von hoch und gering involvierten Konsumenten . . . . . 77

6.1 Übersicht über Vor- und Nachteile verschiedener Befragungsarten . . . . . 125

6.2 Kundenzufriedenheitsmessung . . . . . . . . . . . . . . . . . 127

6.3 Beurteilung der Ladungen der reflektiven Indikatoren . . . . . . . . . . . . 139

6.4 Beurteilung der Gewichte der formativen Indikatoren . . . . . . . . . . . . 140

6.5 Beurteilung der Güte der reflektiven Messmodelle . . . . . . . . . . . . . . 141

6.6 Komponentenmatrix . . . . . . . . . . . . . . . . . . . . . . 142

6.7 Überprüfung der Diskriminanzvalidität . . . . . . . . . . . . . . . . . . . 143

6.8 Klassifikationstabelle für den Wechselvollzug . . . . . . . . . . . . . . . . 146

6.9 Pfadkoeffizienten der direkten Pfade zur Wechselabsicht . . . . . . . . . . . 147

6.10 Pfadkoeffizienten der direkten Pfade zum Wechselvollzug . . . . . . . . . . 148

6.11 Interaktionseffekte im Zusammenhang mit der Wechselabsicht . . . . . . . 151

6.12 Interaktionseffekte im Zusammenhang mit dem Wechselvollzug . . . . . . . 153

6.13 Vergleich der Regressionskoeffizienten der direkten Effekte bei Wechselabsicht und Wechselvollzug . . . . . . . . . . . . . . . . . . . . . 156

6.14 Vergleich der Regressionskoeffizienten der moderierenden Effekte bei Wechselabsicht und Wechselvollzug . . . . . . . . . . . . . . 157 


\section{Abbildungsverzeichnis}

2.1 Zusammenhang zwischen Energieträgern und Nutz-Energien . . . . . . . . 12

2.2 Informiertheit der bundesdeutschen Konsumenten über den Wettbewerb in der Stromwirtschaft im Zeitverlauf . . . . . . . . . . . . . . . . . 13

2.3 Zeitliche Indizierung von Einstellungen . . . . . . . . . . . . . . . 26

3.1 Kernmodell der Theorie des überlegten Handelns . . . . . . . . . . . . . . 37

3.2 Kernmodell der Theorie des geplanten Verhaltens . . . . . . . . . . . . . . 39

3.3 Ein Modell zur Austauschtheorie . . . . . . . . . . . . . . . . . . . . . . 42

4.1 Teilaspekte der Kundenzufriedenheit mit einem Energieversorger . . . . . . 49

4.2 Komponenten globaler Evaluierungen . . . . . . . . . . . . . . . . 52

4.3 Wirkzusammenhang zwischen Dienstleistungsqualität, Kundenzufriedenheit und Verhaltensabsicht . . . . . . . . . . . . . . . . . 54

4.4 Wechselbarrieren und Wechselkosten . . . . . . . . . . . . . . . . 64

4.5 Hypothesensystem der direkten Wirkbeziehungen beim Wechselverhalten von Konsumenten im Markt für Haushaltsstrom . . . . . . . . . . . . . . . 87

4.6 Hypothesensystem der direkten und moderierenden Effekte beim Wechselverhalten von Konsumenten im Markt für Haushaltsstrom . . . . . . . . . 89

5.1 Modell mit zwei latenten Variablen . . . . . . . . . . . . . . . . . . . 93

5.2 Reflektives Messmodell mit drei Indikatoren . . . . . . . . . . . . . . . . 95

5.3 Formatives Messmodell mit drei Indikatoren . . . . . . . . . . . . . . . 96

5.4 Formative und reflektive Indikatoren am Beispiel des Konstrukts „Trunkenheit" . . . . . . . . . . . . . . . . . . . . . 97

5.5 Alternative Spezifikationen von Konstrukten zweiter Ordnung . . . . . . . 100

5.6 Beispiel für ein Modell mit moderierendem Effekt . . . . . . . . . . . . . . 102

5.7 Transkription eines Modells mit moderierendem Effekt . . . . . . . . . . 103

5.8 Der PLS-Algorithmus . . . . . . . . . . . . . . . . . . . . . 105

5.9 Beispiel für ein Modell mit drei latenten Variablen . . . . . . . . . . . . . . 106

5.10 Beispiel für die Modellierung von Konstrukten zweiter Ordnung mittels PLS-Pfadmodellierung . . . . . . . . . . . . . . . . . . . . . 110 
6.1 Zusammensetzung der Stichprobe nach Alter und Geschlecht . . . . . . . . 126

6.2 Messmodell der Kundenzufriedenheit . . . . . . . . . . . . . . . . . . . . . 129

6.3 Potenzielle Ersparnis durch einen Anbieterwechsel in Abhängigkeit vom Stromverbrauch . . . . . . . . . . . . . . . . . . . . . 131

6.4 Index der Attraktivität der Alternativen . . . . . . . . . . . . . . . . . 132

6.5 Messmodell des direkten Aufwands eines Wechsels . . . . . . . . . . . . . . 133

6.6 Messmodell der Such- und Informationskosten . . . . . . . . . . . . . . . . 134

6.7 Messmodell des wahrgenommenen Risikos . . . . . . . . . . . . . . . . . . 134

6.8 Operationalisierung der Wechselkosten als Konstrukt zweiter Ordnung . . . 135

6.9 Messmodell des Involvements . . . . . . . . . . . . . . . . . . . . . . 136

6.10 Messmodell der Wechselabsicht . . . . . . . . . . . . . . . . . . . 137

6.11 Überprüfung der externen Validität der formativen Messung der Attraktivität der Alternativen mittels eines Pseudo-MIMIC-Modells . . . . . . . . 145

6.12 Stärke der direkten Wirkbeziehungen beim Wechselverhalten von Konsumenten im Markt für Haushaltsstrom . . . . . . . . . . . . . . . . . . . . 150

6.13 Signifikante direkte und moderierende Effekte beim Wechselverhalten von Konsumenten im Markt für Haushaltsstrom . . . . . . . . . . . . . . . . . 155

7.1 Ein modifiziertes Modell zur Austauschtheorie . . . . . . . . . . . . . . . 161

7.2 Strategieoptionen eines Anbieters . . . . . . . . . . . . . . . . . 162

7.3 Strategieoptionen eines Anbieters in der Verteidigerposition . . . . . . . . . 168

7.4 Strategieoptionen eines Anbieters in der Angreiferposition . . . . . . . . . 170

7.5 Auswirkung von Marketinginstrumenten auf die Kundenzufriedenheit . . . 173 


\section{Abkürzungsverzeichnis}

\begin{tabular}{|c|c|}
\hline Abb. & Abbildung \\
\hline Abk. & Abkürzung \\
\hline AVE & average variance extracted \\
\hline bspw. & beispielsweise \\
\hline bzgl. & bezüglich \\
\hline bzw. & beziehungsweise \\
\hline ca. & circa \\
\hline CL & Comparison Level \\
\hline $\mathrm{df}$ & degrees of freedom \\
\hline d. h. & das heißt \\
\hline d. Verf. & der Verfasser \\
\hline EMAC & European Marketing Academy \\
\hline engl. & englisch \\
\hline EnWG & Energiewirtschaftsgesetz \\
\hline etc. & et cetera \\
\hline e. V. & eingetragener Verein \\
\hline EVU & Energieversorgungsunternehmen \\
\hline f. & folgende \\
\hline ff. & fortfolgende \\
\hline gew. & gewichtet \\
\hline ggf. & gegebenenfalls \\
\hline i. A. & im Allgemeinen \\
\hline i. d. R. & in der Regel \\
\hline i. e. & that means \\
\hline i.e.S. & im engeren Sinne \\
\hline inkl. & inklusive \\
\hline LV & latente Variable \\
\hline MIMIC & Multiple Indicators and Multiple Causes \\
\hline Nr. & Nummer \\
\hline n.s. & nicht signifikant \\
\hline n. v. & nicht verfügbar \\
\hline
\end{tabular}


OFGEM Office of Gas and Electricity Markets

o. S.

ohne Seitenangabe

PLS

Partial Least Squares

S.

Seite

S.

siehe

Sig.

Signifikanz

sog.

so genannt

Sp.

Spalte

StdErr

Standardfehler

Tab.

Tabelle

taz

die tageszeitung

u. a.

unter anderen $(\mathrm{m})$

USA

United States of America

VDEW

Verband der Elektrizitätswirtschaft

Verf.

Verfasser

vgl.

vergleiche

vS.

versus

z. B.

zum Beispiel

z. T.

zum Teil 


\section{Symbolverzeichnis}

\begin{tabular}{|c|c|}
\hline$\alpha$ & Cronbach's Alpha (Maß der internen Konsistenzreliabilität) \\
\hline$b_{j i}$ & vorläufiger Pfadkoeffizient des Pfads von $\xi_{i}$ nach $\xi_{j}$ \\
\hline$\beta_{j i}$ & Pfadkoeffizient des Pfads von $\xi_{i}$ nach $\xi_{j}$ \\
\hline$C L$ & Vergleichsstandard \\
\hline$C L_{\text {alt }}$ & Vergleichsstandard für Alternativen \\
\hline$D$ & Designmatrix \\
\hline$d_{i j}$ & binäres Element der Designmatrix \\
\hline$\delta_{j}$ & Fehlerterm der formativen latenten Variable $\xi_{j}$ \\
\hline$D Q_{t}$ & transaktionsbezogene Dienstleistungsqualitätsevaluation \\
\hline$e_{j i}$ & Gewicht des Pfads von $\xi_{i}$ nach $\xi_{j}$ für die innere Schätzung \\
\hline$f_{j}$ & Faktoren zur Standardisierung der inneren Schätzer $Z_{j}$ \\
\hline$f^{2}$ & Efektgröße \\
\hline$\varepsilon_{j h}$ & Fehlerterm des $h$-ten Indikators der latenten Variable $\xi_{j}$ \\
\hline$\varphi_{j}$ & Faktoren zur Standardisierung der äußeren Schätzer $Y_{j}$ \\
\hline$g, h=1, \ldots, H_{j}$ & Indizes der Indikatoren der latenten Variable $\xi_{j}$ \\
\hline$H_{j}$ & Anzahl der Indikatoren der latenten Variable $\xi_{j}$ \\
\hline$i, j=1, \ldots, J$ & Indizes der latenten Variablen \\
\hline$J$ & Anzahl der latenten Variablen \\
\hline$k$ & Anzahl der Bootstrapstichproben \\
\hline$\lambda_{j h}$ & Ladung des $h$-ten Indikators der latenten Variable $\xi_{j}$ \\
\hline$\lambda^{2}$ & Indikatorreliabilität \\
\hline$n$ & Anzahl der Versuchspersonen \\
\hline$P_{t}$ & transaktionsbezogene Preisevaluation \\
\hline$P Q_{t}$ & transaktionsbezogene Evaluation der Produktqualität \\
\hline$\pi_{j h}$ & Gewicht des $h$-ten Indikators der latenten Variable $\xi_{j}$ \\
\hline$r_{j g h}=r_{j h g}$ & Korrelation zwischen den Indikatoren $x_{j g}$ und $x_{j h}$ \\
\hline$R^{2}$ & Bestimmtheitsmaß \\
\hline$\rho_{c}$ & Faktorreliabilität \\
\hline$t$ & Index über Transaktionen \\
\hline$x_{j h}$ & $h$-ter Indikator der latenten Variable $\xi_{j}$ \\
\hline$X_{j}=\left(x_{j 1} \cdots x_{j H_{j}}\right)$ & Matrix aus den Indikatoren der latenten Variable $\xi_{j}$ \\
\hline
\end{tabular}




$\begin{array}{ll}\xi_{j} & \text { latente Variable } \\ Y_{j} & \text { äußerer Schätzer für die latente Variable } \xi_{j} \\ v_{j} & \text { Fehlerterm der latenten Variable } \xi_{j} \text { beim Strukturmodell } \\ z_{i}^{W e c} & \text { kontinuierlicher Schätzwert für den Wechselvollzug } \\ Z_{j} & \text { innerer Schätzer für die latente Variable } \xi_{j} \\ Z_{t} & \text { transaktionsbezogene Zufriedenheit } \\ \zeta_{1}, \zeta_{2} & \text { Schwellen zur Dichotomisierung der } z_{i}^{W e c}\end{array}$




\section{Einführung}

Durch die Liberalisierung des Strommarkts für Haushaltsstromkunden erhielten deutsche Konsumenten Ende der 1990-er Jahre erstmals die Möglichkeit, ihren Stromanbieter zu wechseln. Viele neu gegründete Unternehmen witterten die Chance, die Kunden der noch nicht an Wettbewerb gewöhnten Ex-Monopolisten auf einfache Weise abzuwerben. Aufwendige Werbekampagnen verhalfen einigen neuen Anbietern zu hohem Bekanntheitsgrad, und die große Mehrheit der Konsumenten war sich der Möglichkeit bewusst, den Stromanbieter zu wechseln. Doch trotz der objektiv günstigeren Angebote - für viele Konsumenten ließen sich Einsparungen von deutlich über zehn Prozent erzielen - wechselte nur ein Bruchteil der deutschen Haushalte seinen Stromanbieter. Dies überraschte umso mehr, als Umfragen zuvor eine vielfach höhere latente Wechselbereitschaft erfasst hatten. Vor diesem Hintergrund stellen sich zwei Fragen:

- Wie lässt sich allgemein das Wechselverhalten der Konsumenten im Strommarkt erklären?

- Warum setzt speziell von denjenigen Konsumenten, die die Absicht zum Wechseln des Stromanbieters haben, nur ein geringer Anteil seine Absicht um?

Erste Erklärungsansätze aus der Praxis beriefen sich vor allem auf die Trägheit der Konsumenten. Eine tiefergehende wissenschaftliche Auseinandersetzung mit dem Wechselverhalten von Konsumenten im deutschen Strommarkt gibt es bis dato jedoch noch nicht. Diese Lücke möchte die vorliegende Arbeit schließen.

Die Entscheidungsumgebung, in der sich Haushaltsstromkunden befinden, ist nicht nur für den Strommarkt typisch, sondern findet sich auch an anderer Stelle wieder: Konsumenten stehen in einer Versorgungsbeziehung mit einem Versorger, d. h. einem Anbieter eines bestimmten Produkts oder einer Produktgruppe, und sie haben die Möglichkeit, diesen Anbieter zu wechseln. Konsumenten unterhalten eine Vielzahl solcher Versorgungsbeziehungen mit unterschiedlichen Anbietern. Versorgungsbeziehungen werden häufig auf unbestimmte Zeit eingegangen oder verlängern sich selbstständig, sofern nicht einer der Austauschpartner bewusst eine Beendigung der Versorgungsbeziehung herbeiführt. Um diese Versorgungsbeziehungen aufzulösen bzw. den Anbieter zu wechseln, bedarf es einer 
expliziten Entscheidung und Handlung des Konsumenten. Typisch für Versorgungsbeziehungen ist, dass die Wahl des Anbieters und das Abrufen bzw. Nutzen des in dieser Beziehung ausgetauschten Produkts zwei getrennte Entscheidungssituationen darstellen.

Als Beispiele für Versorgungsbeziehungen sind neben der Energieversorgung Personenund Sachversicherungen, Telekommunikationsanschlüsse, Mietwohnungen, Zeitschriftenabonnements und Bankkonten zu nennen. Einerseits lassen sich Erkenntnisse, die in der Marketingforschung bereits über Versorgungsbeziehungen gewonnen wurden, möglicherweise auch auf den Strommarkt für Haushaltsstromkunden übertragen. Hierzu ist eine empirische Überprüfung unerlässlich. Andererseits können hinzugewonnene Erkenntnisse über das Konsumentenverhalten im Strommarkt für Haushaltsstromkunden wiederum hilfreich für die Forschung über andere Versorgungsbeziehungen sein.

Für Anbieterunternehmen stellt das Wechseln von Kunden bei Versorgungsbeziehungen eine Herausforderung dar: Für etablierte Anbieter, die ihre Kunden halten wollen, ist es von großer Bedeutung, die Abwanderung von Kunden gering zu halten oder zu reduzieren. Schließlich ist der bestehende Kundenstamm die Quelle von Unternehmensumsatz und gewinn. Um Kunden halten zu können, ist es für etablierte Anbieter wichtig, ein profundes Verständnis über das Wechselverhalten von Kunden zu erlangen. Hingegen benötigen Anbieter, die Kunden von anderen Anbietern abwerben möchten, ein profundes Verständnis davon, auf welche Weise sie Konsumenten zum Wechseln des Anbieters bewegen können. Wissen über das Wechselverhalten von Kunden und dessen Ursachen ist also für die Marketingplanung unmittelbar nützlich und kann Unternehmen zu Wettbewerbsvorteilen verhelfen.

Zwar zeigte sich in den vergangenen zehn Jahren großes Interesse an der Erforschung andauernder Beziehungen zwischen Unternehmen und ihren Kunden, wie zahlreiche grundlegende Forschungsbeiträge zu Kundenbeziehungsmarketing und Kundenbindung in Konsumgütermärkten bezeugen (vgl. z. B. BerRY, 1995; Gwinner ET AL., 1998; SHeth und Parvatiyar, 1995). Trotz der Bedeutung des Wechselverhaltens von Konsumenten für die Anbieter hat sich jedoch die Marketingwissenschaft erst in sehr geringem Umfang explizit mit Wechselverhalten bei Versorgungsbeziehungen auseinander gesetzt.

Die vorliegende Arbeit soll dazu beitragen, neue Erkenntnisse über das Wechselverhalten von Konsumenten bei Versorgungsbeziehungen zu gewinnen. Als Anschauungsobjekt wird hierbei der Strommarkt für Haushaltsstromkunden dienen.

Der Strommarkt für Haushaltsstromkunden kann als typisch für eine Versorgungsbeziehung angesehen werden: Zwischen einem Konsumenten und einem Anbieter besteht ein Versorgungsvertrag, mit dem sich der Konsument für unbestimmte Zeit auf den Anbieter festlegt. Der eigentliche Bezug erfolgt automatisiert, und der Konsum des Produktes 
Strom ist losgelöst von einer Entscheidung über die Wahl eines Anbieters. Dies wird anhand des Beispiels Haushaltsgeräte deutlich: Einige Geräte sind permanent an das Stromnetz angeschlossen (z.B. Kühlschränke oder Durchlauferhitzer), andere Geräte werden aktiv vom Stromkunden eingeschaltet (z. B. Licht oder Staubsauger). In beiden Fällen ist mit dem Stromkonsum keine explizite Auswahlentscheidung verbunden, da der zu diesem Zeitpunkt liefernde Stromanbieter feststeht.

Abschnitt 1.1 arbeitet heraus, warum das Thema „Wechselverhalten von Konsumenten im Strommarkt" Relevanz besitzt. Abschnitt 1.2 sammelt die aufgeworfenen Fragen und formuliert die Problemstellung der vorliegenden Arbeit. Abschnitt 1.3 erläutert den Aufbau der Arbeit.

\subsection{Relevanz des Themas}

Beim Wechselverhalten von Konsumenten im Strommarkt handelt es sich um ein Phänomen, das für viele Adressaten eine hohe Relevanz besitzt: Stromanbieter benötigen ein profundes Verständnis vom Verhalten ihrer aktuellen und potenziellen Kunden; dies gilt für die systematische Kundenbindung ebenso wie für die Akquisition von Neukunden. Auch staatliche oder überstaatliche Institutionen, die die wirtschaftlichen Rahmenbedingungen gestalten, brauchen Information über das Wechselverhalten der Konsumenten. Insbesondere für Unternehmen, die mit ihren Kunden in Versorgungsbeziehungen stehen, ist der Kundenstamm von essenzieller Bedeutung. So weist u. a. Plinke (1997, S. 115) auf die herausragende Bedeutung der Geschäftsbeziehung für den langfristigen Unternehmenserfolg hin: „Geschäftsbeziehungen mit Kunden sind von allem, was ein Unternehmen ,hat', mit das Wichtigste." Der Kundenstamm eines Unternehmens bestimmt wesentlich dessen Ertragsstärke (vgl. z. B. Heskett et AL., 1991, S. 30ff.; Heskett et AL., 1994, S. 166f.; Belz, 1994, S. 15-19) und Unternehmenswert (Henseler und Hoffmann, 2003). BerRY (1980, S. 25) betont, dass es für die meisten Unternehmen wichtiger sei, Kunden zu haben als Kunden zu gewinnen.

Für einen Anbieter kann die Bindung von Kunden vorteilhafte Motivations-, Wahrnehmungs- und Verhaltenskonsequenzen mit sich bringen (BANSAL und TAYLOR, 1999, S. 200). Darüber hinaus ist es für Anbieter von großer Bedeutung, Kunden an sich zu binden, da die Abwanderung von Kunden mit negativen Konsequenzen wie reduziertem Marktanteil, geschmälerter Profitabilität ${ }^{1}$ und erhöhten Kosten einhergeht (vgl. REICHHeld und Sasser, 1990, S. 105-108; Reichheld, 1993, S. 64; Rust und Zahorik, 1993, S. 211; Rust ET AL., 1995, S. 59). Der Wert eines Kundenstamms hängt nicht nur von

\footnotetext{
1 Vorausgesetzt, die abwandernden Kunden lieferten einen positiven Deckungsbeitrag.
} 
der Anzahl der Kunden ab, sondern auch von der Profitabilität der einzelnen Kunden. Dabei spielen insbesondere auch nicht-monetäre Faktoren eine wichtige Rolle (vgl. PLINKE, 1997, S. 126f.; Homburg und Schnurr, 1998, S. 171ff.; Zezelu, 2000, S. 13f.; CorNELSEN, 2000, S. 30). Unternehmen, die diesen Wert von Kunden vor Augen haben und daher nach der Bindung oder Akquisition von Kunden streben, müssen zur Verwirklichung eines effektiven Marketing-Managements das Wechselverhalten von Kunden analysieren und begreifen.

Viele Produkte, die üblicherweise in Form von Versorgungsbeziehungen vertrieben werden, weisen eine Gemeinsamkeit hinsichtlich ihrer Märkte auf: Ihre Märkte waren in der Vergangenheit sehr stark reglementiert und sind erst vor kurzer Zeit liberalisiert worden. Dies gilt bspw. für Haushaltsstrom ebenso wie für Telefonanschlüsse oder gesetzliche Krankenkassen. Verständlicherweise hatten sich daher in der Vergangenheit weder die Anbieter noch die Kunden mit dem Phänomen des Anbieterwechsels beschäftigt. Gleiches gilt für wissenschaftliche Arbeiten zu diesem Thema: Da sich die Frage nach dem Anbieterwechsel und dessen Konsequenzen bei den meisten Versorgungsbeziehungen (noch) nicht stellte, war auch das Interesse der Marketingwissenschaft gering, eine rein hypothetische Fragestellung zu bearbeiten. Speziell bezogen auf den Strommarkt lassen sich in der Literatur nur wenige Publikationen finden, die sich explizit mit Marketing und Energiewirtschaft auseinandersetzen (KLOUBERT, 2001, S. 3). Zudem basieren diese auf den Rahmenbedingungen eines regulierten, monopolähnlichen Marktumfelds, das in dieser Form nicht mehr der Realität entspricht. So erschöpfen sich die deutschsprachigen Werke zu diesem Themenfeld nahezu in den Arbeiten von Silzer (1959) aus dem Bereich Business-to-Business-Marketing, der verhaltenswissenschaftlich ausgerichteten Arbeit von Clemens (1983) sowie den allgemeineren Arbeiten von Mahlert (1967), HaAs (1975), CAdosch (1982), Brachmann (1985) und LöBbe (1993). Zwar verdeutlichte bereits MAhlert (1967, S. 31), dass „[... ] Marketing auch in der Energiewirtschaft kein Modewort ist, sondern eine marktwirtschaftliche Notwendigkeit." Dies darf aber nicht darüber hinwegtäuschen, dass die vormals existierenden regionalen Monopole nicht dem Leitbild des Marketing entsprachen, d.h. dem „[... ] Austausch in freier Wahl bei fairen Bedingungen unter geschäftsfähigen Partnern zum Wertgewinn beider" (KoTLER und BLIEMEL, 2001, S. 5). Auch die von HaAs (1975, S. 104) propagierte Erweiterung der Marketingfunktion in der Elektrizitätswirtschaft - weg von der allein zeitlichen Verlagerung des Elektrizitätsverbrauchs und der Absatzsteigerung hin zu umfassender Absatzlenkung genügt diesem Leitbild nicht. Erst die Liberalisierung des Strommarkts mit der neuen Freiheit der Konsumenten, ihren Anbieter zu wechseln, implizierte die Notwendigkeit von Marketing - sowohl für die etablierten Stromanbieter, die sich seit der Liberalisierung des Strommarkts in einem stagnierenden Markt mit wachsendem Wettbewerb wiederfin- 
den, als auch für die neu in den Markt eingetretenen Anbieter. Die Liberalisierung führte auch $\mathrm{zu}$ einem wachsenden wissenschaftlichen Interesse an der Energiebranche; davon zeugen die Dissertationen von BAKAY (2003) und KLOUBERT (2001). Im Vergleich mit anderen Branchen, die seit längerer Zeit liberalisiert sind, tritt aber der wissenschaftliche Nachholbedarf deutlich zu Tage. Insbesondere das Konsumentenverhalten von Haushaltsstromkunden mit seinem Kernelement, dem Wechselverhalten, bedarf einer umfassenden wissenschaftlichen Analyse.

\subsection{Problemstellung}

Der vorhergehende Abschnitt demonstrierte die Relevanz, die das Wechselverhalten von Konsumenten im Strommarkt besitzt. Um so mehr erstaunt es, wie wenig Beachtung das Wechselverhalten bislang in der Praktiker-, aber auch in der wissenschaftlichen Literatur gefunden hat. Speziell im Strommarkt streift lediglich die Arbeit von BAKAY (2003), die sich mit dem Phänomen der Kundenbindung beschäftigt, den Themenkomplex Wechselverhalten.

Im Hinblick auf das Defizit in der Marketingforschung zum Wechselverhalten von Konsumenten im Strommarkt stellt sich die zentrale Frage nach der Ursache für das Wechselverhalten. Die erste Forschungsfrage, der sich die vorliegende Arbeit widmet, lautet daher: Was veranlasst Kunden dazu, bei ihrem Stromanbieter zu bleiben bzw. was veranlasst Kunden dazu, ihren Stromanbieter zu wechseln?

In einem ersten Schritt ist zu ermitteln, welche Faktoren das Wechselverhalten von Konsumenten beeinflussen. In einem zweiten Schritt ist $\mathrm{zu}$ untersuchen, auf welche Weise diese Faktoren die Entscheidung beeinflussen, den Anbieter zu wechseln.

Ziel dieser Arbeit ist es also, das Phänomen „Wechselverhalten von Konsumenten im Strommarkt" zu erklären. Zu beachten ist hierbei, dass die Begriffe „Wechselverhalten“, „Kundenbindung“ und „Kundenloyalität“ eng miteinander verwandt sind. Während Kundenbindung und Kundenloyalität sich für einen Anbieter positiv auswirken, hat der Wechsel eines Kunden zu einem anderen Anbieter negative Folgen (BANSAL und TAYLOR, 1999, S. 200) - beides unter der Prämisse, dass der betrachtete Kunde für den Anbieter profitabel ist.

Diese Arbeit beschränkt sich auf die Untersuchung des Wechselverhaltens von Konsumenten, d. h. privaten Haushaltsstromkunden, die Strom konsumieren und weder mit ihm handeln noch ihn als Faktor in einen Produktionsprozess einfließen lassen. Bei diesen Kunden kann man das Phänomen beobachten, dass sie trotz einer bestehenden Absicht, ihren 
Stromanbieter zu wechseln, ihre Absicht häufig nicht in die Tat umsetzen. Verschiedene Studien schätzen, dass bei ca. 15 bis $20 \%$ der deutschen Haushalte eine latente Bereitschaft zum Wechseln des Stromanbieters vorliegt (vgl. MEYer und Dornach, 2000, S. 135; O. V., 2000, S. 25; Datamonitor, 1999, S. 100-104). Demgegenüber liegt der Anteil derjenigen Haushalte, die ihren Anbieter gewechselt haben, bei weniger als fünf Prozent (VDEW, 2002b, S. 4). Warum setzt also nur ein geringer Teil derjenigen, die den Willen zum Wechsel haben, ihren Willen um? Hierfür haben bisher weder die Marketingwissenschaft noch Praktiker empirisch gestützte Erklärungen vorgelegt. Ein maßgeblicher Grund für dieses Defizit ist, dass die Mehrzahl der bestehenden Studien zum Kundenverhalten in Versorgungsbeziehungen, so auch die Arbeit von BAKAY (2003) zur Kundenbindung von Haushaltsstromkunden, allein Verhaltensintentionen oder die Verbundenheit mit dem Anbieter als relevante endogene Größe untersucht. Das realisierte Verhalten der Konsumenten bleibt jedoch unberücksichtigt. Im Rahmen dieser Arbeit soll daher eine weitere Forschungsfrage beantwortet werden: Inwieweit unterscheiden sich die Einflussgrößen der Absicht von Konsumenten, den Stromanbieter zu wechseln, von den Einflussgrößen des Wechselvollzugs?

Um beide Forschungsfragen zu beantworten, soll in dieser Arbeit ein Modell entwickelt werden, das nicht nur aufzeigt, welche Variablen in welcher Form auf die Absicht von Kunden zum Wechseln ihres Anbieters einwirken, sondern auch Erkenntnisse darüber liefert, welche Variablen den tatsächlichen Vollzug eines Wechsels auf welche Weise beeinflussen. Der empirische Test dieses Modells soll zum einen Rückschlüsse auf die Wirkstärke und Form der Wirkung unterschiedlicher Variablen ermöglichen. Zum anderen kann ein Vergleich der Determinanten von Wechselabsicht und Wechselvollzug möglicherweise Aufschluss darüber geben, warum Konsumenten, die eine Wechselabsicht besitzen, ihren Anbieter doch nicht wechseln. Das zu entwickelnde Modell spiegelt die Sichtweise des Kunden wider. Aus dem empirisch untermauerten Modell sollen sich Handlungsempfehlungen für die verschiedenen Marktteilnehmer ableiten lassen: Etablierte Anbieter erhalten damit einen Fingerzeig, auf welche Stellgrößen sie sich konzentrieren sollten, um der Abwanderung von profitablen Kunden entgegenzuwirken; neue Anbieter erhalten Unterstützung bei der Neukundenakquise.

\subsection{Aufbau der Arbeit}

Das nachfolgende Kapitel 2 stellt zunächst den Strommarkt für Haushaltsstromkunden und Versorgungsbeziehungen als Untersuchungsobjekte vor. Dann liefert eine Literaturauswertung einen Überblick über die bisher bekannten Erkenntnisse über Wechselverhal- 
ten bei Versorgungsbeziehungen. Der letzte Abschnitt des zweiten Kapitels widmet sich gesondert der Trägheit von Konsumenten als beliebtem Erklärungsansatz.

Kapitel 3 führt in Theorien und Konzepte ein, die einen Erklärungsbeitrag zum Wechselverhalten von Konsumenten in Versorgungsbeziehungen leisten können. Den Anfang bilden die Lerntheorie der instrumentellen Konditionierung und ein Konzept von BLIEMEL und EGGERT (1998b), nach dem Motivationen zur Fortsetzung von Beziehungen anhand der beiden Dimensionen „Wille zur Fortsetzung“ und „Zwang zur Fortsetzung“ kategorisiert werden. Weiterhin werden die Theorie der kognitiven Dissonanz, die Theorie überlegten Handelns und die auf ihr aufbauende Theorie geplanten Verhaltens sowie die Austauschtheorie vorgestellt.

Kapitel 4 greift sowohl auf die vorgestellten Theorien als auch die bestehende empirische Forschung zurück und identifiziert vier Konstrukte, die für die Erklärung des Wechselverhaltens bei Versorgungsbeziehungen maßgeblich erscheinen: Die Kundenzufriedenheit mit dem aktuellen Anbieter, die Attraktivität der Alternativen, die wahrgenommenen Wechselkosten und das Involvement. Die vier Konstrukte werden jeweils konzeptualisiert. Für jedes der Konstrukte werden Hypothesen über die Wirkbeziehungen im Rahmen des Wechselverhaltens von Konsumenten bei Versorgungsbeziehungen aufgestellt. Die gewonnenen Hypothesen werden zu einem Hypothesensystem von direkten und moderierenden Effekten zusammengefügt.

Kapitel 5 beschreibt das methodische Handwerkszeug für die spätere quantitative Analyse des Hypothesensystems. Nach Ausführungen über den Aufbau von Strukturgleichungsmodellen im Allgemeinen wendet sich das Kapitel der Schätzung und Validierung von PLSPfadmodellen zu. In diesem Zusammenhang wird auch eine neue Methode zur Schätzung von Interaktionseffekten vorgestellt.

Kapitel 6 unterzieht das in Kapitel 4 entwickelte Hypothesensystem einer empirischen Überprüfung. Hierfür erfahren die maßgeblichen Konstrukte zunächst eine geeignete Operationalisierung, bevor die direkten und moderierenden Effekte zwischen ihnen analysiert werden.

Kapitel 7 behandelt zunächst die Implikationen für die Marketingtheorie. Sodann leitet es aus den gewonnenen Erkenntnissen Handlungsempfehlungen für die Marketingpraxis ab. Anschließend werden die Grenzen dieser Arbeit und Vorschläge für darauf aufbauende Forschung diskutiert.

Zum Abschluss fasst Kapitel 8 die vorliegende Arbeit zusammen. 


\section{Untersuchungsobjekt und bestehende Erklärungsansätze}

Ziel dieses Kapitels ist es, ein Fundament für die weitere Untersuchung zu legen. Hierzu bedarf es einer Fixierung des Forschungsobjekts und der Aufbereitung des aktuellen Forschungsstands. Den Anfang bildet eine Darstellung des Strommarkts für Haushaltsstromkunden (Abschnitt 2.1). Auf einen Abriss der geschichtlichen Entwicklung folgt die Analyse der gegenwärtigen Situation. Abschnitt 2.2 trägt die bestehenden Erkenntnisse zu Wechselverhalten bei Versorgungsbeziehungen zusammen. Hierzu wird zunächst auf das Wesen von Versorgungsbeziehungen eingegangen, bevor dann der Stand der Marketingwissenschaft zu Wechselverhalten bei Versorgungsbeziehungen anhand der bisher durchgeführten qualitativen und quantitativen empirischen Studien aufgezeigt wird. Abschnitt 2.3 durchleuchtet die bestehende Literatur nach Anhaltspunkten für die Unterschiede zwischen Wechselabsicht und Wechselvollzug.

Als eine mögliche Ursache der geringen Wechselrate im deutschen Strommarkt für Haushaltskunden wird in der Öffentlichkeit die Trägheit der Konsumenten diskutiert. Abschnitt 2.4 arbeitet heraus, dass es sich bei dieser Argumentation entweder um eine Tautologie oder ein Synomym für den Verbleib in Versorgungsbeziehungen aufgrund hoher wahrgenommener Wechselkosten oder eines zu niedrigen Involvements handelt.

\subsection{Der Strommarkt für Haushaltsstromkunden}

Am 19. Februar 1882 wurde mit der Versorgung eines Stadtteils von Berlin erstmals elektrischer Strom in nennenswertem Umfang Gegenstand unternehmerischen Handelns (MANN und LAKER, 1997, S. 463). In der Folge begannen die zahlreich entstehenden kommunalen und regionalen Stromanbieter, abgesteckte Gebiete mit Strom zu versorgen. Bis Ende der 1990er Jahre besaßen die etwa 1000 deutschen Stromversorger für Konkurrenten geschlossene Versorgungsgebiete, so dass Stromkunden einer monopolistischen Marktsituation ausgesetzt waren. Zwischen den Stromanbietern und den jeweiligen Gebietskörperschaften bestanden sog. Demarkationsverträge. Darin räumten die Gebietskörperschaften gegen Zahlung einer Konzessionsabgabe einem Stromanbieter das alleinige Recht der Stromlieferung innerhalb eines festgelegten Gebiets ein (KLOPfer und Schulz, 1993, 
S. 4). Wettbewerb herrschte lediglich in Form eines Substitutionswettbewerbs zwischen Energieträgern wie z. B. Gas, Öl und Kohle (FrITZ und KÖNIG, 2000, S. 3). Diese regionalen Monopole erklären sich nicht nur aus der Wirtschaftsgeschichte, sondern lassen sich auch theoretisch begründen: Bei Strom handelt es sich um einen leitungsgebundenen Energieträger, d. h. die Energie gelangt mit Hilfe einer Transportinfrastruktur zu den Kunden. Strom aus Batterien oder mobilen Generatoren findet im Rahmen dieser Arbeit keine Beachtung. Wied-Nebbeling (1993, S. 37) sowie Borrmann und Finsinger (1999, S. 101ff.) zeigen, dass sich aus der Notwendigkeit der Transportinfrastruktur die Eigenschaft eines natürlichen Monopols ableitet. Hierbei ist eine etwaige doppelte Verlegung von Leitungen durch einen Wettbewerber ineffizient, solange die bestehenden Leitungen noch freie Kapazität aufweisen.

Die seit mehr als 100 Jahren bestehende Wettbewerbssituation im Strommarkt hat sich seit 1998 zunächst für Industrie- und später auch für Haushaltsstromkunden grundlegend geändert: der Strommarkt wurde liberalisiert. Die Liberalisierung des Strommarkts ist auf die Initiative der Europäischen Union zurückzuführen, die schließlich in die EURichtlinie für Elektrizität mündete. Zur Umsetzung der EU-Richtlinie beschloss der Bundestag 1997 das Gesetz zur Neuregelung des Energiewirtschaftsrechts, das am 29. April 1998 in Kraft trat. Dieses Gesetz beendete die bis dahin existierenden Wettbewerbsbeschränkungen durch Demarkations- und Konzessionsverträge der Energieversorger (vgl. Eickhof, 1998, S. 437; Fritz und KöNIG, 2000, S. 7-9).

KlOpfer und Schulz (1993, S. 9, 53-57) postulierten als grundlegende Elemente der Liberalisierung die Beseitigung des Gebietsschutzes, den Zugang Dritter zu den Netzen, die Entflechtung vertikal integrierter Unternehmen hinsichtlich Organisation, Eigentum und Buchungskreis sowie die Liberalisierung von Kraftwerks- und Leitungsbau. Diese Elemente der Liberalisierung fanden zum großen Teil ihre Umsetzung. Sie wirkten sich auf unterschiedliche Weise auf das Marketing im Strommarkt aus. Tab. 2.1 zeigt eine zusammenfassende Darstellung der marketingspezifischen Auswirkungen der Deregulierung. Dies sind im Einzelnen (KLOUBERT, 2001, S. 30f.):

- Aus vormals geschlossenen Versorgungsgebieten wurde ein Markt mit Wettbewerb. Ausgehend von den Regelungen des Energiewirtschaftsgesetzes (vgl. §§ 5-8 EnWG) wurde in Deutschland das Wettbewerbsmodell des verhandelten Netzzugangs (engl.: negotiated third party access) etabliert. Hierbei verhandeln verschiedene Verbände der Elektrizitätswirtschaft über Durchleitungsgebühren und Zählermieten. Dieses Wettbewerbsmodell ermöglicht es zum einen Stromanbietern, elektrische Energie in ein Fremdnetz einzuspeisen, und zum anderen Kunden, Strom von einem anderen als dem regionalen Stromanbieter zu beziehen. 
Regulierte Energiewirtschaft

Geschlossene Versorgungsgebiete

Kostenorientierter Strompreis

Abnehmer-Versorger-Beziehung

Reaktion

Energieträger

\section{Deregulierte Energiewirtschaft}

Markt

Marktpreise

Kundenbeziehung

Aktion

Energieträger und Dienstleistung

Tabelle 2.1: Auswirkungen der Deregulierung der Energiewirtschaft (in Anlehnung an Kloubert, 2001, S. 30)

- Der Austausch von Strom in Kundenbeziehungen wird mehr und mehr durch Marktpreise anstatt durch kostenorientierte Strompreise geprägt.

- Sprachen Stromanbieter vor der Liberalisierung noch von Abnehmern oder Versorgungsfällen, so sehen sie sich nun Kunden gegenüber.

- Während ehemals die passiv orientierte Versorgung von Abnehmern mit Strom im Vordergrund stand, müssen nun kundenorientierte Angebote über aktives, unternehmerisches Handeln zu Erlösen führen.

- Das Produkt Strom wird zunehmend mit Dienstleistungen augmentiert. ZinnBauer (2001, S. 249) nennt als Möglichkeiten der Produktaugmentation bspw. Festnetzund Mobiltelefonie, Sicherheitsdienste und Dienstleistungen rund um den Hausbau.

Der Wandel der Rahmenbedingungen hat weitreichende Konsequenzen für die etablierten Versorger (Beutin ET AL., 2001, S. 171). Für sie stellt sich in zunehmendem Maße die Frage nach Kundenorientierung, Kundenzufriedenheit und Kundenbindung (PAYNE und Frow, 1997, S. 463). Solange der Wettbewerb um Kunden noch durch Demarkationsverträge ausgeschlossen wurde, ließen sich Kundenbeziehungen als Konstante ansehen (KLOuBerT, 2001, S. 2); durch die Liberalisierung wurden Kundenbeziehungen zu Variablen. Viele andere Charakteristika des Strommarkts blieben jedoch unverändert: Konsumenten verstehen die Versorgung mit Energie als lebensnotwendige Grundversorgung, die von ihren bisherigen Versorgern normalerweise mit großer Zuverlässigkeit erfüllt wurde (Wagner und Kristof, 2002, S. 439). Die Geschäftsbeziehungen sind daher durch Aspekte wie Vertrauen, Schutz, aber auch Abhängigkeit geprägt. Das zentrale Produkt Strom ist aufgrund seiner Normierung ein homogenes Gut (HEGER, 2003, S. 246). Laut 
Wagner und Kristof (2002, S. 426) sind „Energieträger [..] für den Nutzer immer nur Mittel zum Zweck." Menschliche Bedürfnisse sind nicht unmittelbar auf Energie wie Strom oder Erdgas gerichtet. Die Nachfrage nach Energie leitet sich erst ab aus Bedürfnissen wie hellen und warmen Räumen, gekühlten Lebensmitteln oder Mobilität. WAGNER und Kristof (2002, S. 426) zeigen, dass die Bedürfnisbefriedigung durch Energieträger in einem mehrstufigen Prozess geschieht (vgl. Abb. 2.1). Dass Strom nur indirekt zur Bedürfnisbefriedigung beiträgt, hat Konsequenzen: Viele Autoren sehen Strom allgemein als ein Produkt an, mit dem Kunden sich nicht befassen wollen, also ein Low-Interestbzw. Low-Involvement-Produkt (vgl. z. B. Keller und Matzke, 2000, S. 55; SchoenHeit und Niedergesëss, 1999, S. 465; Seiferth, 1999, S. 388; Krick, 1999, S. 58). Zinnbauer (2001, S. 243) bringt es auf den Punkt: „Strom stellt [.. ein typisches LowInterest-Produkt dar." Eine solche Betrachtung unterstellt, dass der Grad des Involvements zwar für verschiedene Produkte bzw. Produktkategorien unterschiedlich ist, jedoch über die Konsumenten hinweg nahezu homogen verteilt ist.

Eng mit dem Involvement verwandt ist die Informiertheit der Konsumenten über das Produkt Strom und über die Möglichkeit, den Stromanbieter zu wechseln. Eine Betrachtung wichtiger Kundeneinstellungen im Zeitablauf, u. a. der Informiertheit der Konsumenten in Deutschland über den Wettbewerb in der Stromwirtschaft, ermöglichen die seit 1999 jährlich durchgeführten Kundenfokus-Studien des Verbands der Elektrizitätswirtschaft (VDEW). Die durchschnittliche Informiertheit über den Wettbewerb in der Stromwirt-

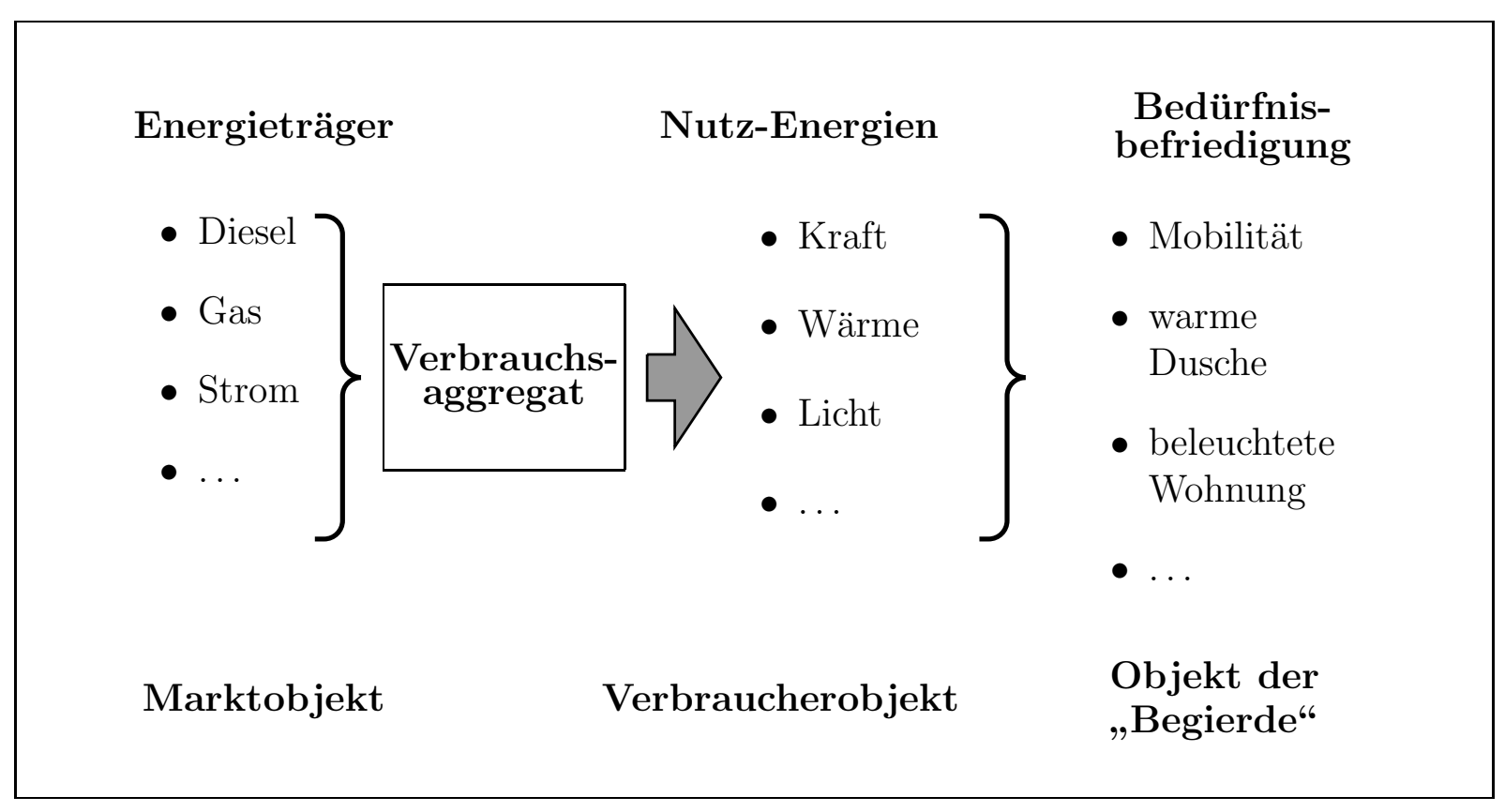

Abbildung 2.1: Zusammenhang zwischen Energieträgern und Nutz-Energien. Angelehnt an Wagner und Kristof (2002, S. 427) 
Durchschnittliches Niveau der Antworten der befragten Stromkunden auf die Frage: „Wie gut fühlen Sie sich über den Wettbewerb in der Stromwirtschaft informiert?"

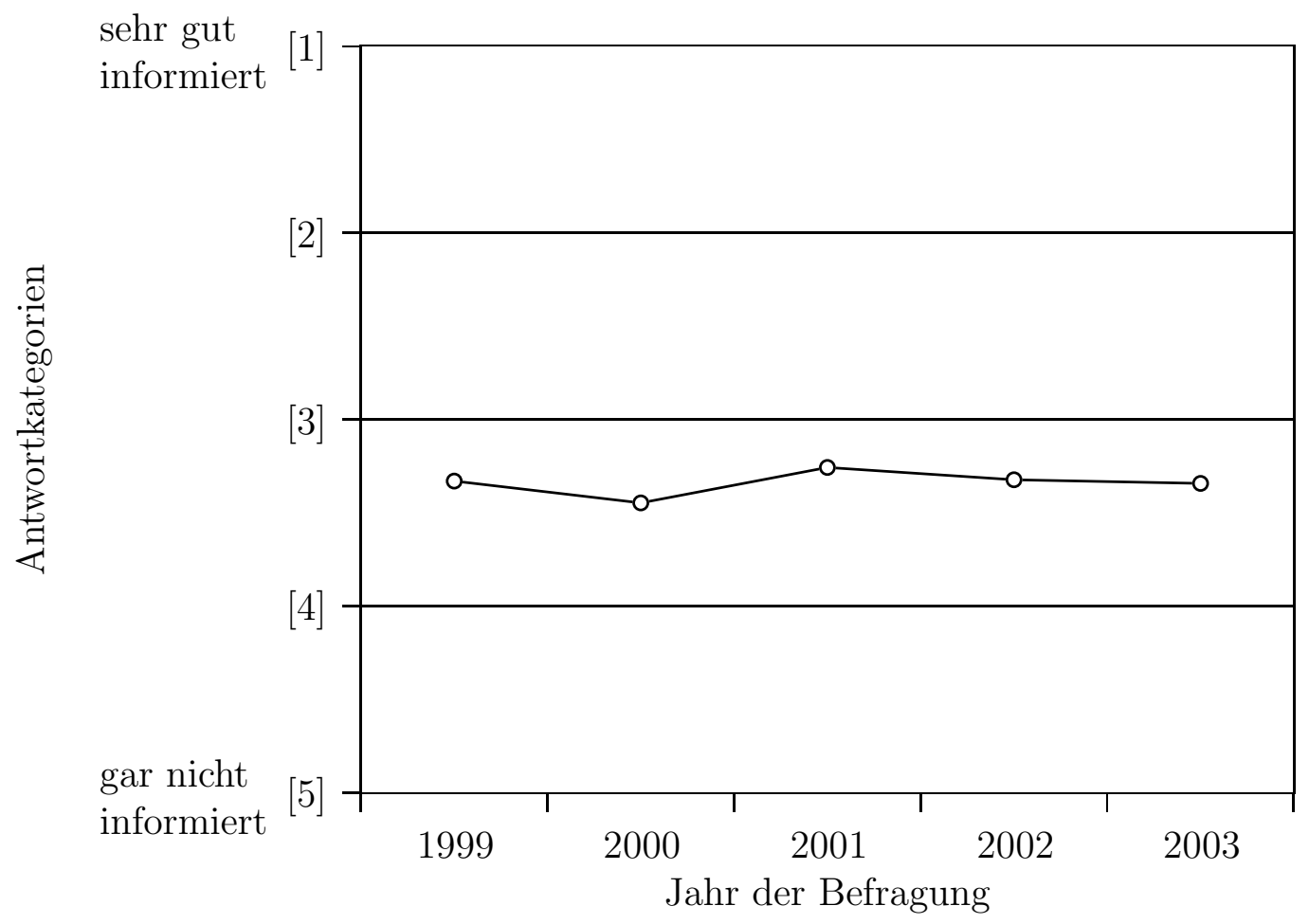

Abbildung 2.2: Informiertheit der bundesdeutschen Konsumenten über den Wettbewerb in der Stromwirtschaft im Zeitverlauf. Durchschnittliche Bewertung der Versuchspersonen. Eigene Darstellung aus Daten der VDEWKundenfokus-Studien 2000-2003

schaft hat sich in den Jahren 1999 bis 2003 nur in geringem Umfang verändert, wie aus Abb. 2.2 hervorgeht. Nach einer leichten Abnahme der Informiertheit zwischen den Jahren 1999 und 2000 stieg die Informiertheit zum Jahr 2001 an und bewegte sich in den Folgejahren wieder langsam auf das Ausgangsniveau von 1999 zu. Die Änderung der durchschnittlichen Informiertheit war nur zwischen den Jahren 2000 und 2001 signifikant (Scheffé-Test), dort allerdings mit einer Irrtumswahrscheinlichkeit von weniger als einem Promille.

Vielfach wurde angenommen, dass sich der Wettbewerb in den Energieversorgungsmärkten ähnlich entwickeln würde wie im Telekommunikationsmarkt (LAKER ET AL., 1998, S. 50). So erwarteten bspw. LAKER und Herr (2000, S. 18ff.), dass die Liberalisierung des Energieversorgungsmarkts nicht nur zu grundlegenden Veränderungen in der Wettbe- 
werbsstruktur und bei den angebotenen Versorgungsleistungen, sondern auch - zumindest mittelfristig - zu bedeutenden Änderungen der Kundenbedürfnisse führen würde. Laut Pirovsky (1999, S. 36) rechnete man zu Beginn der Liberalisierung mit einer raschen Marktentwicklung mit Wechselraten zwischen 10 und $20 \%$.

Die Konsumenten nahmen die Angebote neuer Anbieter jedoch in weit geringerem Umfang an als dies die Unternehmen und die Regulierungsbehörde erwartet hatten (KLOUBERT, 2001, S. 1). Obwohl Verbraucher ihren Stromanbieter frei wählen können, und trotz massiver Werbung in Fernsehen und Hörfunk, auf Plakaten und in Printmedien, hat die Wechselbereitschaft der Verbraucher nicht signifikant zugenommen (PIMPL, 1999, S. 32). Laut VDEW (2002b) wechselten zwischen dem Beginn der Liberalisierung im Jahr 1998 und Ende 2001 etwa 1,4 Millionen Haushalte den Stromanbieter (ohne Umzüge), das entsprach 3,7\% der 39 Millionen deutschen Haushalte. Die Wechselbereitschaft an sich ist relativ hoch: So haben ca. $27 \%$ der privaten Haushalte darüber nachgedacht, den Stromanbieter zu wechseln (VDEW, 2000, S. 33). Nicht nur absolut, sondern auch im europäischen Vergleich sind die deutschen Wechselraten niedrig (KOMMISSION DER EUROPÄISCHEN Union, 2001). Einen anderen Verlauf nahm die Marktentwicklung bspw. in Großbritannien: Dort wechselten seit der Liberalisierung des Energieversorgungsmarktes $38 \%$ aller Haushalte ihren Stromanbieter (OFGEM, 2002, S. 19).

Als möglichen Grund für die niedrige Wechselrate identifizieren GRAEHL ET AL. (2001, S. 227) Probleme beim Anbieterwechsel; darunter fallen insbesondere Versuche der etablierten Anbieter, durch langwierige Wechselverfahren Konkurrenzangebote für die Kunden unattraktiv zu machen. Wiedmann und Walsh (2003, S. 225) nennen des Weiteren Trägheit der Kunden, Suchkosten, Risikoaversion sowie mangelnde Markttransparenz aus der Sicht der Endkunden als potenzielle Ursachen für die niedrige Wechselrate.

Die bislang geringe Wechselrate bringt ein generell niedriges Erfahrungsniveau hinsichtlich der Versorgungssicherheit unterschiedlicher Anbieter mit sich (BAKAY, 2003, S. 22). Konsumenten können daher kaum auf Erfahrungen anderer Konsumenten als Entscheidungshilfe beim Anbieterwechsel zurückgreifen (KLOUBERT, 2001, S. 99).

\subsection{Wechselverhalten bei Versorgungsbeziehungen}

Aufgrund der spärlichen empirischen Befunde zum Wechselverhalten von Konsumenten im Strommarkt - allein die Arbeit von BAKAY (2003) liefert Erkenntnisse zur Kundenbindung von Haushaltsstromkunden - betrachtet dieser Abschnitt die vorliegenden Erkentnisse zu Wechselverhalten in anderen Branchen. Die Betrachtung beschränkt sich auf 
Versorgungsbeziehungen, d.h. Austauschbeziehungen, die vom Charakter her den Kundenbeziehungen im Strommarkt nah verwandt sind. Es ist zu erwarten, dass Erkenntnisse zum Wechselverhalten bei anderen Versorgungsbeziehungen sich als hilfreich für die Modellierung des Wechselverhaltens von Konsumenten im Strommarkt erweisen.

Unterabschnitt 2.2.1 stellt Versorgungsbeziehungen als einen Spezialfall von Kundenbeziehungen vor und überprüft, für welche Produkte Versorgungsbeziehungen typisch sind. In den darauffolgenden Unterabschnitten wird ein Blick auf empirische Studien geworfen, die sich mit den Determinanten von Wechselverhalten, Wechselabsicht, Kundenbindung oder Kundenloyalität bei Versorgungsbeziehungen auseinander setzen: Unterabschnitt 2.2.2 gibt zunächst einen Überblick über die explorativen Studien, die nach Determinanten des Wechselverhaltens bei Versorgungsbeziehungen suchten. Im Anschluss befasst sich Unterabschnitt 2.2.3 mit den eher konfirmatorischen Studien, die sich vorwiegend auf die Überprüfung von Hypothesen zu Wechselverhalten bei Versorgungsbeziehungen konzentrieren.

\subsubsection{Versorgungsbeziehungen}

Was sind Versorgungsbeziehungen und wo findet man sie vor? Um sich dieser Frage zu nähern, beschäftigt sich dieser Unterabschnitt zunächst mit Geschäftsbeziehungen im Allgemeinen, bevor Versorgungsbeziehungen als Spezialfall beschrieben werden.

Beziehungen werden in der Literatur auf verschiedene Weise definiert. Einige Wissenschaftler nehmen eine sehr weite Perspektive ein, indem sie sämtliche wiederkehrenden Interaktionen zwischen Käufer und Verkäufer ohne Berücksichtigung der Wiederkaufmotive als Beziehungen bezeichnen (vgl. stellvertretend für viele DAY, 2000, S. 24f.). Diese Definition knüpft an die sozialpsychologische Sichtweise von Beziehungen als einer Sequenz von Interaktionen an (HINDE, 1979, S. 15). Laut Hinde (1979, S. vi ff.) zeichnet sich eine Beziehung durch drei Eigenschaften aus (vgl. auch LudwIG, 2000, S. 124-126): Interaktion, Dauerhaftigkeit und Integrativität.

Beispielsweise enthalten typische Geschäftsbeziehungen zwischen einem Stromanbieter und einem Konsumenten eine Reihe von unterschiedlichen Interaktionen: KLOUBERT (2001, S. 16) nennt als Beispiele für Interaktionen die Marktkommunikation eines Stromanbieters, die Rechnungstellung, die kontinuierliche Leistungserstellung, die Bearbeitung von Kundenanfragen zu Serviceleistungen oder die Mitteilungen von Leistungsstörungen. Diesen Aktionen von Anbietern steht jeweils eine Aktion aus Kundensicht gegenüber, bspw. die Perzeption von Werbung, die Kontrolle der Periodenabrechnung, der Abruf der angebotenen Leistung etc. Die Dauerhaftigkeit findet sich im abgeschlossenen Versor- 
gungsvertrag wieder, die Verbindung über das Stromnetz macht die Integrativität deutlich.

Allgemein sind Beziehungen in erster Linie zwischen Familienmitgliedern und Freunden zu finden, erst in zweiter Linie knüpfen Konsumenten kommerzielle Beziehungen zu Organisationen und deren Mitarbeitern (IAcobucci und Ostrom, 1996) oder auch zu Marken (Fournier, 1998). Im Zentrum der Untersuchung stehen dyadische Beziehungen, d. h. Beziehungen zwischen genau zwei Elementen. Im konkret vorliegenden Fall von dyadischen Geschäftsbeziehungen handelt es sich um die Relation zweier Marktsubjekte: Kunde und Anbieter. DAY (2000, S. 24) weist darauf hin, dass im Mittelpunkt einer jeden Geschäftsbeziehung ein Austauschprozess steht, in dem Wert gegeben und empfangen wird. Austausch umfasst hierbei „[...] die Menge der Aktivitäten, die auf die Anbahnung, Durchführung und Kontrolle eines wechselseitig bedingten Transfers von Verfügungsrechten zwischen zwei oder mehr Parteien gerichtet sind" (Plinke, 2000, S. 9). Ein wechselseitig bedingter Transfer von Verfügungsrechten kommt zu Stande, wenn eine Seite etwas leistet oder anbietet (Verfügungsrechte über ein Produkt) in der Erwartung, dafür im Gegenzug etwas von der anderen Seite zu erhalten. Die Untersuchung von Geschäftsbeziehungen fügt sich auf diese Weise in die wissenschaftliche Tradition der Marketingforschung ein, in der der Austausch das zentrale Explanandum darstellt (s. z. B. BAGOzZI, 1975).

Von der Vielzahl von Austauschbeziehungen, die Konsumenten mit unterschiedlichen Anbietern unterhalten, zeichnen sich einige dadurch aus, dass das Abrufen oder Nutzen der in diesen Beziehungen ausgetauschten Produkte losgelöst von der Wahl des Anbieters erfolgt. Werden solche Austauschbeziehungen auf unbestimmte Zeit eingegangen oder verlängern sie sich selbstständig, sofern nicht einer der Austauschpartner bewusst eine Beendigung der Austauschbeziehung herbeiführt, lässt sich von Versorgungsbeziehungen sprechen. Um diese Versorgungsbeziehungen aufzulösen bzw. den Anbieter zu wechseln, bedarf es einer expliziten Entscheidung und Handlung des Konsumenten.

In der deutschsprachigen betriebswirtschaftlichen Literatur wurden Versorgungsbeziehungen als solche weder definiert noch eingehend erörtert. Für die vorliegende Arbeit soll die folgende Definition für Versorgungsbeziehungen gelten: Versorgungsbeziehungen sind auf unbestimmte Zeit eingegangene Beziehungen zwischen Kunden und Anbietern, in denen sich der Kunde verpflichtet, ein Produkt immer bei einem bestimmten Anbieter zu festgelegten Konditionen zu beziehen, und der Anbieter sich verpflichtet, das Produkt dem Kunden bereitzustellen, ohne dass es dafür einer Kontaktaufnahme oder Auswahlentscheidung durch den Kunden bedarf.

Dieser Definition liegt der weit gefasste Produktbegriff von KotLer und Bliemel (2001, S. 14) zu Grunde, d. h. „[e]in Produkt ist alles, was einer Person angeboten werden kann, 
um ein Bedürfnis oder einen Wunsch zu befriedigen." Es stellt sich die Frage, welche Produkte typischerweise das Austauschobjekt in Versorgungsbeziehungen sind.

Sucht man in der marketingwissenschaftlichen Literatur nach Arbeiten zu Versorgungsbeziehungen, so stößt man schnell auf den von BoLton (1998) geprägten Begriff „continuously provided services", also kontinuierlich erbrachte Dienstleistungen. Sie subsummiert darunter bestimmte Gesundheitsdienstleistungen, Versicherungen, Telekommunikation, Finanzdienstleistungen sowie Ver- und Entsorgungsleistungen (BoLTon, 1998, S. 46). Versorgungsbeziehungen haben sich jedoch nicht nur bei Dienstleistungen entwickelt; man findet sie auch - wenngleich seltener - bei Sachgütern. Eine der wenigen Formen von Versorgungsbeziehungen bei Sachgütern stellen Abonnements dar: Abonnements sind eine traditionelle Vertriebsform, die bei Zeitungen und Zeitschriften dominiert, aber auch bei anderen Produkten zu beobachten ist. Versorgungsbeziehungen findet man auch gelegentlich bei Heimlieferungsdiensten, die Kunden in regelmäßigen Abständen mit bestimmten Sachprodukten wie bspw. Milch oder Brötchen beliefern. Als weiterer Gütertyp sind Rechte zu nennen, bei denen es ebenfalls Beispiele für Versorgungsbeziehungen gibt. So stellt z. B. ein unbefristetes Mietverhältnis über Wohnraum, also das Recht zum Gebrauch des Wohnraums, eine Versorgungsbeziehung dar. Ähnlich wie Sachgüter spielen auch Rechte eine eher untergeordnete Rolle gemessen an der Gesamtzahl von Versorgungsbeziehungen, die Konsumenten im Durchschnitt unterhalten.

Zusammenfassend lässt sich festhalten, dass es über alle Gütertypen hinweg Produkte gibt, die über Versorgungsbeziehungen ausgetauscht werden, wobei Dienstleistungen eine dominante Stellung einnehmen. Bei manchen Produkten besteht für den Anbieter und/

\begin{tabular}{|c|c|c|c|c|}
\hline \multirow{2}{*}{$\begin{array}{l}\text { Art des } \\
\text { Austauschs }\end{array}$} & \multicolumn{4}{|c|}{ Güterarten } \\
\hline & $\begin{array}{l}\text { Sach- } \\
\text { leistungen }\end{array}$ & $\begin{array}{l}\text { Dienst- } \\
\text { leistungen }\end{array}$ & $\begin{array}{l}\text { Energie- } \\
\text { leistungen }\end{array}$ & Rechte \\
\hline $\begin{array}{l}\text { diskrete } \\
\text { Transaktionen }\end{array}$ & $\begin{array}{l}\text { z. B. } \\
\text { Zeitung } \\
\text { am Kiosk }\end{array}$ & $\begin{array}{l}\text { z. B. } \\
\text { Devisen- } \\
\text { umtausch }\end{array}$ & $\begin{array}{l}\text { z. B. } \\
\text { Strom in } \\
\text { Batterien }\end{array}$ & $\begin{array}{l}\text { z. B. } \\
\text { Park- } \\
\text { gebühr }\end{array}$ \\
\hline $\begin{array}{l}\text { Versorgungs- } \\
\text { beziehung }\end{array}$ & $\begin{array}{l}\text { z. B. } \\
\text { Zeitung } \\
\text { im Abo }\end{array}$ & $\begin{array}{l}\text { z. B. } \\
\text { Giro- } \\
\text { konto }\end{array}$ & $\begin{array}{l}\text { z. B. } \\
\text { Strom aus } \\
\text { dem Netz }\end{array}$ & $\begin{array}{l}\text { z. B. } \\
\text { Stellplatz- } \\
\text { miete }\end{array}$ \\
\hline
\end{tabular}

Tabelle 2.2: Arten von Gütern und Austauschbeziehungen 
oder den Nachfrager die Alternative, das Produkt entweder in Form von einzelnen Transaktionen oder über Versorgungsbeziehungen auszutauschen. Tab. 2.2 zeigt eine Gütertypologie nach Koppelmann $(1969,1997)$ in Verbindung mit diskreten Transaktionen oder Versorgungsbeziehungen und führt Beispiele für die verschiedenen Kombinationen von Gütertypen und Austauscharten an. Diese Tabelle verdeutlicht, dass Versorgungsbeziehungen quer über alle Gütertypen hinweg vorkommen.

Die folgende Aufzählung trägt Produkte zusammen, die gewöhnlich über Versorgungsbeziehungen ausgetauscht werden. Sie soll zur Illustration dienen und erhebt keinen Anspruch auf Vollständigkeit:

- Der (leitungsgebundene) Bezug von Strom, Gas, Wasser oder Fernwärme,

- Versicherungen (sofern nicht zeitlich beschränkt wie z. B. für eine Reise),

- zahlreiche Finanzdienstleistungen wie Bankkonten, Vermögensverwaltung, Kredite und Kreditkarten,

- die Entsorgung von Müll und Abwasser,

- Telekommunikationsanschlüsse für Telefon oder Internet,

- Kabelfernsehen,

- gemieteter (Wohn-)Raum sowie

- Abonnementzeitungen und -zeitschriften.

Die folgenden beiden Unterabschnitte tragen die empirischen Studien zusammen, die sich mit Wechselverhalten von Kunden bei Versorgungsbeziehungen befassen. Als Selektionskriterium dienen die Produktkategorien, die soeben als für Versorgungsbeziehungen typisch identifiziert wurden.

\subsubsection{Empirische Studien mit explorativem Charakter zu Versorgungsbeziehungen}

KeAveney (1995) berichtet über die Ergebnisse einer Critical-Incident-Studie unter Dienstleistungskunden zu den Gründen für Wechselverhalten bei Versorgungsbeziehungen. Sie identifiziert acht Hauptursachen für Wechselverhalten: Fehler bei der Kerndienstleistungserbringung, Fehler beim Kundenkontakt, Fehler im Zusammenhang mit dem Preis, Reaktionen auf Dienstleistungsfehler, Unbequemlichkeiten, Angebote von Wettbewerbern, ethische Probleme sowie unfreiwilliges Wechselverhalten (KEAVENEY, 1995, S. 74-78): 
1. Fehler bei der Kerndienstleistungserbringung spielen in der Untersuchung von KEAVENEY die größte Rolle. Es kann sich dabei um einfache Fehler, Abrechnungsfehler oder auch Fehler mit katastrophalen Folgeauswirkungen handeln.

2. Nah verwandt damit sind Fehler beim Kundenkontakt, wenn die Mitarbeiter des Anbieters Kunden vernachlässigen, oder ihr Verhalten durch Unhöflichkeit, Unempfänglichkeit für Kundenwünsche oder Unvermögen gekennzeichnet ist.

3. Eine dritte wichtige Ursache für Wechselverhalten ist der Preis. Kunden wechseln, wenn der letztlich zu zahlende Preis Referenzpreise übersteigt. Referenzpreise können interne normative Preise, der mit der Leistungserbringung erhaltene Wert, Konkurrenzpreise, ehemalige Preise beim gleichen Anbieter oder im Vorhinein abgegebene Kostenvoranschläge sein.

4. Unangemessene Reaktionen auf Dienstleistungsfehler sind eine weitere Wechselursache. In solchen Fällen gingen Mitarbeiter nur widerwillig auf Kunden ein oder ignorierten Kundenbeschwerden völlig. Im schlimmsten Fall bezichtigten Mitarbeiter die Kunden der Verursachung der Probleme.

5. Unbequemlichkeiten nehmen Kunden in Form von unpassenden Öffnungszeiten, schlechten Unternehmensstandorten oder langen Wartezeiten wahr.

6. Angebote von Wettbewerbern, die durch eine höhere Qualität oder einen erweiterten Leistungsumfang hervorstechen, bewegen Konsumenten dazu, den Anbieter zu wechseln.

7. Ethische Probleme beziehen sich auf illegales, unmoralisches, unehrliches, opportunistisches, unsicheres, ungesundes, beschämendes oder sonstiges Verhalten, das weit von der sozialen Norm abweicht.

8. Unfreiwilliges Wechselverhalten beruht vorwiegend auf Faktoren, die Konsumenten nicht oder kaum zu beeinflussen vermögen, wie bspw. Unternehmenszusammenschlüsse und -konkurse oder ein Umzug des Konsumenten oder des Anbieters.

Gerrard und Cunningham (2004) untersuchen, inwieweit einzelne Vorkommnisse im Rahmen der Dienstleistungserbringung das Wechselverhalten von Bankkunden beeinflussen. Dabei gelangen sie zu dem Ergebnis, dass sich das Wechselverhalten von mehr als $90 \%$ der Kunden auf die folgenden Ursachen zurückführen lässt (GERRARD und CUNNINGHAM, 2004, S. 221): 
- Die wichtigste Rolle spielt die Preisgestaltung, die sich aufgrund des komplexen Leistungsangebots von Banken sowohl in der Höhe der Bankgebühren für die verschiedenen Dienstleistungen als auch in den Zinssätzen für Guthaben und Kredite niederschlägt.

- An zweiter Stelle stehen Fehler bei der Dienstleistung, die sich auf das Servicepersonal oder das Produkt an sich zurückführen lassen.

- Als dritte wichtige Ursache für Wechselverhalten sind Unbequemlichkeiten zu nennen. Speziell bei Banken handelt es sich dabei um die Verfügbarkeit der Dienstleistung in zeitlicher und geographischer Hinsicht.

STEWART (1998) betrachtet das Wechselverhalten von Kunden in Form des „exit process in banking“. Sie identifiziert dabei vier Typen von Vorkommnissen als maßgebliche Ursachen des Wechsels der Bank (STEWART, 1998, S. 8):

- „charges and their implementation“,

- „facilities and their availability“,

- „provision of information and confidentiality“ sowie

- „services issues relating to how customers are treated“.

Lubin (1992, S. 25f.) erklärt die Bindung von Kunden zu einem der wichtigsten Marketingziele von Banken. Er zitiert eine Studie, nach der Kunden vor allem aus zwei Gründen ihre Bank wechseln: Zum einen wegen des Personals der Bank, zum anderen wegen eines eingeschränkten Zugriffs auf die Dienstleistungen der Bank.

Bliemel und EGgert (1997) stellen das Konzept der „opportunity mindedness“ vor. Sie verstehen darunter „[d]as intrinsisch motivierte Bestreben[,] neue oder bessere Möglichkeiten der Bedürfnisbefriedigung zu finden und zu nutzen" (BLIEMEL und EGGERT, 1997, o. S.). Opportunity Mindedness ist ein kognitiver Mechanismus, der angesichts einer limitierten geistigen Verarbeitungskapazität die Aufmerksamkeit auf neue und/oder bessere Möglichkeiten zur Bedürfnisbefriedigung lenkt. BLIEMEL und EGGERT sehen die Opportunity Mindedness als verwandt oder gar identisch mit hohem Involvement an.

\subsubsection{Empirische Studien mit konfirmatorischem Charakter zu Versorgungsbeziehungen}

Dieser Unterabschnitt widmet sich empirischen Studien, die die Überprüfung von Hypothesen über Wechselverhalten oder Kundenloyalität bei Versorgungsbeziehungen zum 
Ziel haben. Eine Rekapitulation dieser Studien kann Aufschluss darüber geben, welche Konstrukte sich in anderen Branchen als signifikante Antezedenzen von Wechselverhalten oder Kundenloyalität erwiesen haben. Bei den Untersuchungen zur Kundenloyalität ist zu beachten, dass nicht immer zwischen einer einstellungs- und einer verhaltensorientierten Konzeptualisierung von Kundenloyalität unterschieden wird. Während verhaltensorientierte Konzeptualisierungen von Kundenloyalität nichts anderes als Wechselverhalten darstellen, beziehen sich einstellungsorientierte Konzeptualisierungen auf „, [..] a disposition to continue in a particular exchange relationship based solely on the experiences of the past relationship; that is, regardless of the consequences to the actor" (CzEPIEL und Gilmore, 1987, S. 91).

BeErli ET AL. (2004, S. 265) stellen ein Modell zur Erklärung der Kundenloyalität im Privatkundenbankgeschäft auf. Als wichtigster Einflussfaktor erweist sich die Kundenzufriedenheit, gefolgt mit großem Abstand von wahrgenommenen Wechselkosten. Ein weiteres Modell zur Erklärung der Loyalität von Bankkunden entwickelte LOHMANN (1997). In diesem Modell spielt die Kundenzufriedenheit die größte Rolle, gefolgt von der Bequemlichkeit als einer gewohnheitsnahen Handlungsdisposition. Weiterhin umfasst sein Modell die Konstrukte Vertrauen und Commitment als Erklärungsgrößen (LoHmann, 1997, S. 155). Hallowell (1996, S. 33) kann anhand einer großen Stichprobe im Privatkundenbereich in der Bankindustrie aufzeigen, dass Kundenzufriedenheit mit größerer Kundenloyalität einhergeht. Darüber hinaus korrelieren Kundenzufriedenheit und Kundenloyalität deutlich mit der Profitabilität (HALlowell, 1996, S. 37).

Chakravarty ET AL. (2004) untersuchen, inwieweit neben demographischen Variablen unterschiedliche Dimensionen von Dienstleistungsqualität die Wahrscheinlichkeit beeinflussen, dass Konsumenten die Bank wechseln wollen. Dabei gelangen sie - möglicherweise aufgrund von Multikollinearitätsproblemen - zu folgenden, in Teilen widersprüchlichen Ergebnissen: Einerseits zeichnen sich wechselwillige Kunden im Vergleich zu wechselunwilligen Kunden dadurch aus, dass sie ihre Bank als weniger zuverlässig, weniger entgegenkommend, weniger einfühlend und schlechter ausgestattet ansehen (CHAKRAVARTY ET AL., 2004, S. 518). Andererseits ziehen die Autoren aus einem Regressionsmodell den Schluss, dass lediglich Zuverlässigkeit, Entgegenkommen und Einfühlungsvermögen die Wechselneigung reduzieren, während eine höhere Qualität der Ausstattung tendenziell (allerdings nicht signifikant) sogar die Wechselneigung erhöht (CHAKRAVARTY ET AL., 2004, S. 521f.).

Aus einer industrieökonomischen Sicht zeigt Stango (2002), wie unterschiedliche Formen von Wechselkosten dazu beitragen, dass Konsumenten ihren Kreditkartenanbieter nicht wechseln. Anderson und Sullivan (1993) entdeckten eine negative Beziehung zwischen 
Wechselkosten und der Empfindsamkeit von Konsumenten auf Kundenzufriedenheitsniveaus. Eine Untersuchung von Sharma und PATterson (2000) im Bereich der privaten Vermögensberatung deutet darauf hin, dass Wechselkosten die Wirkbeziehungen von Kundenvertrauen und Kundenzufriedenheit auf das Kundencommitment positiv moderieren.

FREYLAND ET AL. (1999) finden als Einflussgrößen der Loyalität von Versicherungskunden psychische und persönlichkeitsbezogene Determinanten. Psychische Determinanten umfassen hierbei Kundenzufriedenheit sowie psychische Wechselbarrieren im Sinne von Vertrauen und Verbundenheit. Unter persönlichkeitsbezogenen Determinanten subsummieren sie die Ausbildung, das Alter, das Geschlecht, die Nationalität, die Bequemlichkeit, das Wissen und das Interesse. Eine weitere Studie zur Erklärung der Kundenbindung in der Versicherungsbranche unternahmen HeRrmann ET AL. (2000). Als dominierende Determinante erweist sich demnach die Kundenzufriedenheit. Weitere Einflussgrößen sind Wechselbarrieren - von den Autoren als vertrauensvolle, innere Verbundenheit operationalisiert (HERRMANN ET AL., 2000, S. 305) - und die relative Qualität der angebotenen Leistung. Herrmann ET AL. (2000) entwickeln ihr Modell ausgehend von der Theorie des geplanten Verhaltens. Einen ebenfalls auf der Theorie des geplanten Verhaltens basierenden Ansatz wählen BAnsal und TAYLor (1999). Sie entwickeln ein Modell des Wechselverhaltens von Konsumenten bei Dienstleistungen, das sie in der kanadischen Hypothekenbranche überprüfen. Schlesinger und Schulenburg (1995) zeigen anhand des deutschen Markts für Automobilversicherungen, dass die Preissuche, die Produktqualität und die Wechselkosten die Entscheidungen der Kunden über einen Anbieterwechsel prägen.

Gerpott und RAms (2000) stellen ein kausalanalytisches Modell zur Erklärung der Kundenbindung und Kundenloyalität im Mobilfunkmarkt auf. Sie können damit einen direkten Einfluss der Kundenzufriedenheit, der Bewertung von Preisen, des persönlichen Nutzens, der Rufnummernkonstanz und des Wettbewerberimages nachweisen. Hierbei ist zu beachten, dass die vorherrschenden Typen von Mobilkommunikationsverträgen eine Sonderform von Versorgungsbeziehungen darstellen. Sie unterscheiden sich von anderen Versorgungsbeziehungen insofern, als dass die Privatkunden bei vertraglichen Geschäftsbeziehungen nach einer im Vorhinein festgelegten Zeitspanne explizit über die Fortsetzung oder Beendigung der Geschäftsbeziehung entscheiden können. Als üblich hat sich eine Vertragsdauer von 24 Monaten durchgesetzt, während der Privatkunden ihren Mobilfunkvertrag nicht kündigen können. Diese Frist führt u. a. dazu, dass Konsumenten am Vertragsende bewusst über eine Verlängerung des bestehenden Vertrags nachdenken.

BAKAY (2003) untersuchte die Kundenbindung von Haushaltstromkunden in Deutschland. Dabei konnte er insbesondere einen großen Einfluss des individuellen Produktin- 
volvements auf die Kundenbindung (aus Kundensicht) nachweisen. Geringere Auswirkungen auf die Kundenbindung zeigten die Kundenzufriedenheit sowie das von ihm in die Verhaltenswissenschaft eingeführte Konstrukt der „Scheu kognitiver Aufwendungen“.

Es lässt sich das Fazit ziehen, dass die Kundenzufriedenheit und nah verwandte Konstrukte wie die Dienstleistungsqualität eine dominierende Rolle als Erklärungsansätze spielen. In zweiter Linie sind Wechselkosten in unterschiedlichen Erscheinungsformen zu nennen.

\subsection{Ursachen für die Diskrepanz zwischen Wechselabsicht und -vollzug}

Große Diskrepanzen zwischen der Verhaltensabsicht und dem tatsächlichen Verhalten sind nicht nur beim Wechselverhalten im Strommarkt für Haushaltskunden zu beobachten. So konstatieren AJZEN ET AL. (2004, S. 1108): „It is a common observation that people often fail to act in accordance with their stated intentions." Die Diskrepanz zwischen Verhaltensabsichten und Verhalten ist ein bekanntes Problem in der Konsumentenverhaltensforschung: „Although intentions are a significant predictor of behavior, they are far from being a perfect predictor" (BLACKWELL ET AL., 2001, S. 284).

Während die Handlung (im vorliegenden Fall ist dies der Wechselvollzug) sich als primitives Konstrukt ansehen lässt, verbleibt festzulegen, was genau unter einer Verhaltensabsicht zu verstehen ist. Nach Kroeber-RIEL und Weinberg (2003, S. 54) drückt die Verhaltensabsicht „die Absicht aus, in einer bestimmten Situation so oder so zu handeln.“ Eine ausführlichere Definition gibt TrIAndis (1980, S. 203). Er bezeichnet Verhaltenabsichten als „instructions people give to themselves to behave in certain ways. They involve ideas such as ,I must do $X^{6}$, ,I will do $X^{6}$, and ,I am going to do $X^{6}$." Im Rahmen der vorliegenden Arbeit interessiert besonders die Wechselabsicht. Die Wechselabsicht ist eine spezielle Verhaltensabsicht, nämlich die Absicht, den Anbieter zu wechseln.

Verhaltensabsichten lassen sich gut für die Prognose eines Verhaltens einsetzen, da sie „nahe" am Verhalten liegen (Kroeber-Riel und Weinberg, 2003, S. 176). Belk (1985, S. 1) erhebt Verhaltensabsichten zum besten verfügbaren Prädiktor für Verhalten. Die Logik dahinter ist einfach: Wie soll ein Außenstehender das Verhalten eines Konsumenten besser vorhersagen können als der Konsument selbst? Dennoch gibt es Diskrepanzen zwischen Verhaltensabsichten und realisiertem Verhalten. Das im Strommarkt für Haushaltsstromkunden beobachtete Phänomen der Diskrepanz zwischen Wechselabsicht und Wechselvollzug ist keine Ausnahme, sondern eher die Regel: RYAN und BonfIELD (1975) untersuchten 16 Studien, in denen sowohl die Verhaltensabsicht als auch das realisierte 
Verhalten erhoben wurde. Der Median der Korrelationen zwischen der Verhaltensabsicht dem realisierten Verhalten betrug 0,41, d. h. bei der Hälfte der betrachteten Studien erklärte die Verhaltensabsicht weniger als $17 \%$ der Varianz des Verhaltens. BELK (1985, S. 3) kommt zu dem Schluss, dass die Diskrepanz tendenziell sogar noch unterschätzt wird. Wo rührt die Diskrepanz her?

Zunächst ist es wichtig, die Diskrepanz zu konkretisieren: Genau genommen handelt es sich in allen Untersuchungen um Unterschiede zwischen der gemessenen Verhaltensabsicht und dem gemessenen Verhalten. Die Vorhersagekraft der Verhaltensabsicht variiert stark mit dem Verhalten, auf das sie sich bezieht: Wenn eine Person bspw. immer die gleiche Kaffeemarke kauft, so dürfte die Verhaltensabsicht, diesen Kaffee beim nächsten Einkauf wieder zu kaufen, ein zutreffendes Bild der Realität liefern. Allgemein lässt sich regelmäßig wiederholtes Verhalten mit größerer Präzision vorhersagen als sporadisches oder einmaliges Verhalten (BLACKWELL ET AL., 2001, S. 286). Zur letzteren Gattung ist das Wechselverhalten zu zählen.

Peter und Olson (1999, S. 143) identifizieren sieben teilweise miteinander verflochtene Gründe für Diskrepanzen zwischen Absichtsbekundungen und realisiertem Verhalten:

- unvorhergesehene Umwelteinflüsse,

- neue Information,

- die Zwischenzeit,

- Unterschiede in der Konkretheit,

- einen unvorhergesehenen situativen Kontext,

- den Grad der Willenskontrolle sowie

- die Stabilität der Absichten.

Diese sieben Gründe werden im Folgenden eingehender betrachtet.

Unvorhergesehene Umwelteinflüsse, d.h. Faktoren außerhalb des Gestaltungsspielraums des Konsumenten, können die Ausführung der beabsichtigten Handlung vereiteln. Unvorhergesehene Umstände können auch eine Absichtsänderung hervorrufen. CoTE ET AL. (1985) erklären hiermit durchschnittlich 13,6\% der Inkonsistenz zwischen Absicht und Verhalten.

Konsumenten erhalten möglicherweise neue Information über die Konsequenzen der Handlung. So merkt Manski (1990, S. 935) an, dass „even if individuals have rational expectations and stated intentions are best predictions of behavior, intentions and 
behavior need not coincide. The two may converge whenever the information available to respondents at the time of the survey is more limited than the information they possess at the later time when behavior is determined." Neue Information führt mitunter zu Veränderungen der Vorgrößen der Verhaltensabsicht, also insbesondere der Einstellung zur Handlung. Diese Veränderungen bewirken ihrerseits eine Modifikation der Absicht. Die ursprüngliche Absicht ist dann für die Handlung nicht mehr von Belang und stellt keine gute Vorhersage des Verhaltens mehr dar.

Des Weiteren hängt die Vorhersagegenauigkeit von Verhaltensabsichten von der Zwischenzeit ab: Je größer das Zeitintervall zwischen der Messung der Verhaltensabsicht und dem Eintritt des Verhaltens ist, desto mehr Gelegenheiten ergeben sich für ein Individuum, seine Absicht zu ändern (Blackwell ET AL., 2001, S. 286). Hinzu kommt: Je mehr Zeit zwischen der Messung von Absichten und der Beobachtung von realisiertem Verhalten liegt, desto mehr Faktoren erhalten die Gelegenheit, auf die Absicht einzuwirken und sie zu verändern. So können Personen bspw. in der Zwischenzeit etwas lernen, was sie ihre zuvor bekundete Absicht aufgeben lässt. Die Zwischenzeit beeinflusst also die Stärke der beiden zuvor genannten Ursachen.

Eine weitere Ursache für eine Diskrepanz zwischen Verhaltensabsicht und Verhalten können Unterschiede in der Konkretheit sein, d.h. die Messung der Verhaltensabsicht korrespondiert nicht vollständig mit dem zu prognostizierenden Verhalten (BLACKWELL ET AL., 2001, S. 286): So kann es sein, dass eine zeitlich nicht näher bestimmte Verhaltensabsicht gemessen und dann zusammen mit einem bis zu einem konkreten Zeitpunkt beobachteten Verhalten analysiert wird. Neben Unterschieden im Zeithorizont zwischen Absichts- und Verhaltensmessung können auch andere Unterschiede, bspw. in der Spezifität des Verhaltens, auftreten. Die Vorhersagekraft von Verhaltensabsichten wird umso schwächer, je weniger die Verhaltensabsichtsmessung mit dem zu prognostizierenden Verhalten korrespondiert.

Konsumenten können sich, wenn es um die Ausführung des Verhaltens geht, in einem unvorhergesehenen situativen Kontext befinden. Bisweilen unterscheidet sich der situative Kontext, den Konsumenten bei der Absichtsmessung im Hinterkopf haben, vom situativen Kontext der Verhaltensausübung. WARshaw (1980b) schreibt einen Teil der Diskrepanz zwischen Verhaltensabsicht und Verhalten der fehlenden Kontextspezifität der Verhaltensabsichtsmessungen zu. Am Beispiel von Softdrinks zeigt er auf, dass die bekundeten Verhaltensabsichten von Konsumenten eine präzisere Vorhersage des Verhaltens ermöglichen, wenn die Verhaltensabsicht in Abhängigkeit von unterschiedlichen Kontexten erfragt wird. BeLK (1985, S. 7) weist jedoch darauf hin, dass spätere Arbeiten im Allgemeinen keine oder nur leichte Verbesserungen der Vorhersage von Verhalten gezeigt haben. 
Es ist offensichtlich, dass der Grad der Willenskontrolle entscheidend dafür ist, inwieweit Individuen Verhaltensabsichten in Verhalten umsetzen können. Nicht alle Verhaltensarten unterliegen vollständig der Kontrolle durch den Willen. Dies ist insbesondere der Fall, wenn Konsumenten durch nicht beeinflussbare Faktoren an der Ausübung ihres Willens gehindert werden oder die Handlung nicht kognitionsgesteuert erfolgt wie bspw. bei Reflexen.

Die Stabilität der Absichten kann ebenfalls zu Diskrepanzen zwischen einer bekundeten Absicht und realisiertem Verhalten führen. Während einige Absichten sehr stabil sind und auf einer festen Einstellungsstruktur fußen, basieren andere Absichten auf eher schwachen Meinungen und sind daher leichter veränderbar. Genau genommen bestimmen die zu einem bestimmten Zeitpunkt gemessenen Einstellungen nicht direkt eine spätere Verhaltensabsicht oder ein Verhalten. Vielmehr sind zwei Schlüsse zu ziehen: erstens von den aktuellen Einstellungen auf zukünftige Einstellungen und zweitens von Einstellungen (über die Verhaltensabsicht) auf das Verhalten (Kroeber-RIEL und Weinberg, 2003, S. 178). Abb. 2.3 verdeutlicht diesen Zusammenhang.

Die vorangegangenen Erklärungsansätze für Diskrepanzen zwischen Verhaltensabsichten und Verhalten implizierten zum großen Teil intrapersonelle Diskrepanzen. Eine andere Erklärung gibt CAMPBELL (1963): Er unterscheidet dazu zwischen dem hypothetischen Kontext, in dem Personen ihre Absicht bekunden sollen, und dem realen Kontext, in dem es um die Realisierung des Verhaltens geht. Die Reaktionen in beiden Kontexten stellen nach seinem Vorschlag zwei Indikatoren für ein und dieselbe zu Grunde liegende

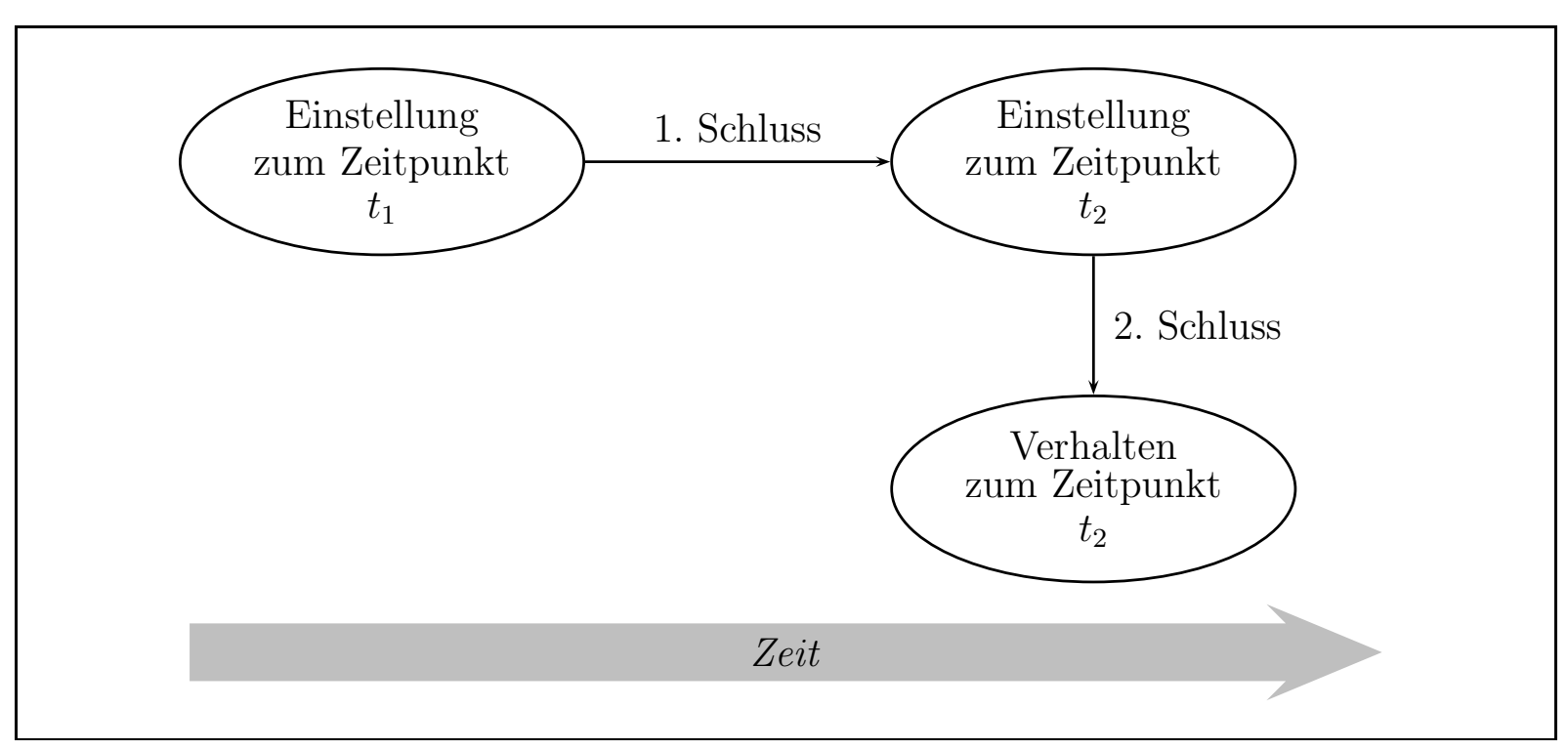

Abbildung 2.3: Zeitliche Indizierung von Einstellungen. Angelehnt an KroEBER-RIEL und WeinBerg (2003, S. 178) 
latente Disposition dar. Von Personen mit deutlich positiven Dispositionen ist zu erwarten, dass sie sowohl in hypothetischen als auch in realen Kontexten wie erwünscht reagieren, wohingegen Personen mit deutlich negativen Dispositionen sowohl in hypothetischen als auch in realen Kontexten zurückhaltend reagieren. Die Diskrepanz zwischen Verhaltensabsicht und Verhalten lässt sich laut CAMPBELL auf Personen mit moderaten Dispositionen zurückführen: Diese Personen reagieren positiv beim hypothetischen Kontext, jedoch negativ beim anspruchsvolleren realen Kontext. Bei der Diskrepanz zwischen Verhaltensabsicht und Verhalten handelt es sich eher um einen Scheineffekt als um eine wirkliche Verzerrung - oder, um mit den Worten CAMPBELls zu sprechen, um einen Fall von Pseudo-Inkonsistenz. Die von CAMPBELL entwickelte Hypothese der latenten Disposition impliziert, dass Individuen, deren Verhaltensabsicht und Verhalten nicht korrespondieren, sich hinsichtlich ihrer latenten Disposition von den übrigen Individuen unterscheiden. AJZEn ET AL. (2004) können die Hypothese der latenten Disposition empirisch belegen.

\subsection{Sind Konsumenten zu träge, um den Anbieter zu wechseln?}

Sowohl Vertreter von Energieversorgungsunternehmen als auch Verbraucherschützer argumentieren, dass die Trägheit von Konsumenten ursächlich dafür ist, dass Konsumenten ihren Anbieter nicht wechseln. ${ }^{1}$ Auch die betriebswirtschaftliche Anwendungsforschung identifiziert die Trägheit der Konsumenten als Ursache des zurückhaltenden Wechselverhaltens (z. B. WiEDMAnN und WALsh, 2003, S. 225). Die Trägheit als eine dem Kunden inhärente Größe stellt eine vermeintlich elegante Erklärung für die geringe Wechselrate im deutschen Strommarkt dar. Kann Trägheit also einen Erklärungsbeitrag zum Wechselverhalten von Konsumenten bei Versorgungsbeziehungen liefern?

Die Trägheit von Konsumenten wurde bereits 1935 von KALDOR thematisiert, der in diesem Kontext von „buyer's inertia“ sprach. SELTEN (1965) verwendet den Begriff „Nachfrageträgheit“. Dabei hat der Begriff der Nachfrageträgheit „nichts anderes zum Inhalt als die Ungültigkeit der in der herkömmlichen Theorie üblichen Annahme, daß die Reaktionsgeschwindigkeit der Nachfrager unendlich groß ist" (SELten, 1965, S. 203).

Um die Rolle der Trägheit im Zusammenhang mit dem Wechselverhalten von Konsumenten bei Versorgungsbeziehungen zu erforschen, bedarf es zunächst einer Festlegung, was genau unter Trägheit zu verstehen ist. In der Marketingwissenschaft und benachbarten Disziplinen haben sich unterschiedliche Definitionen herausgebildet. Sie lassen sich grob in drei Kategorien einteilen:

\footnotetext{
1 Im Abschnitt A.3 des Anhangs sind Quellenfragmente abgedruckt, die dies belegen.
} 
- Trägheit als ein Nicht-Handeln, ein Alles-beim-Alten-Lassen, obwohl ein Handeln oder Verändern für das Individuum vorteilhaft wäre,

- Trägheit als eine unmittelbare Reaktion auf den Aufwand, den eine Handlung mit sich bringt, sowie

- Trägheit als Handeln unter fehlendem Involvement.

Zur ersten Kategorie zählen die Definitionen von Chintagunta (1998), Hirshleifer und Welch (2001), Kruse (2000), Arkes et Al. (2002), Huff Et Al. (1992) sowie Cattell und Winder (1952). Chintagunta (1998, S. 253) definiert Trägheit als positives Abhängigkeitsverhältnis zu einer Marke, in dem Sinne, dass der frühere Kauf einer Marke $X$ die Wahrscheinlichkeit des Wiederholungskaufs erhöht. HiRshLEIFER und Welch (2001, S. 1ff.) definieren Trägheit als Reaktion auf fehlende oder verloren gegangene Information; als höhere Wahrscheinlichkeit, alten Verhaltensweisen zu folgen, als es bei vollem Gedächtnis der Fall wäre. Kruse (2000, S. 123) beschreibt die „emotionale Trägheit“ als Tendenz, im gleichen Zustand zu bleiben. Ähnlich wie Kruse (2000) setzen Arkes ET AL. (2002, S. 372) Trägheit in Form von „inaction inertia“" mit dem NichtErgreifen einer Chance gleich. Huff ET AL. (1992, S. 56) sehen in Trägheit „the tendency to remain with the status quo". Die Psychologen Cattell und Winder (1952, S. 23) sehen in Trägheit „a failure to 'adapt', by the use of a shorter behavioral route than the usual one to a given goal, when circumstances make the shorter route possible."

Unter die zweite Kategorie fällt die Definition von BeErli et AL. (2004). BeERLi ET AL. (2004, S. 255) stellen den Zusammenhang zwischen Trägheit und Suchkosten her: „Inertia means the consumer is buying the same brand, not because of true brand loyalty, but because it is not worth the time and trouble to search for an alternative."

Eine dritte Kategorie bildet die Definition von HuAng und Yu (1999): Wenn sie von Trägheit sprechen, verstehen sie darunter, dass „consumers' [sic] repeatedly purchase the same brand passively without much thought" (HuANG und YU, 1999, S. 525). Der Kerngedanke ist hierbei das Handeln, ohne sich mit der Sache zu befassen, d. h. ein Handeln unter niedrigem oder fehlendem Involvement.

Neben den in der Literatur vorgenommenen Definitionen bzw. Konzeptualisierungen der Trägheit sind auch und gerade die Operationalisierungen der Trägheit aufschlussreich, d. h. wie haben empirische Arbeiten die Trägheit gemessen? Zunächst ist zu beachten, dass der Begriff „Trägheit" im allgemeinen Sprachgebrauch eher negativ besetzt ist (RoEMER, 2004, S. 4). Daher könnte bei der Messung der Trägheit durch Selbstauskunft von Versuchspersonen der Effekt der sozialen Erwünschtheit auftreten, d. h. die Versuchspersonen 
geben keine wahrheitsgemäßen Auskünfte, sondern antworten, wie es als sozial erwünscht erscheint - in diesem Fall also, nicht träge zu sein. Möglicherweise auch aufgrund dieser Problematik reduzieren sich die Versuche, Trägheit durch Befragung von Versuchspersonen zu messen, auf die Arbeiten von Herrmann Et AL. (2000) und Bakay (2003). Herrmann ET AL. (2000, S. 304) operationalisieren das Konstrukt Handlungskontrolle mit einem Indikator des Inhalts „, [z] u bequem sein, um sich um Versicherung zu kümmern“. Offensichtlich misst dieser Indikator die Bequemlichkeit. Bequemlichkeit ist aber nichts anderes als die Präferenz der Option „Nicht-Handeln“, weil die Option „Handeln“ mit $\mathrm{zu}$ hohen Anstrengungen (im Kontext kontinuierlicher Austauschbeziehungen sind das Wechselkosten) verbunden ist. BAKAY (2003) vermeidet den Begriff „Trägheit“ und führt stattdessen das Konstrukt „Scheu kognitiver Aufwendungen“ neu in die Marketingwissenschaft ein, indem er es erstmals operationalisiert. BAKAY (2003, S. 99) entwickelt eine Skala zur Messung der Scheu kognitiver Aufwendungen, die u. a. die folgenden Indikatoren beinhaltet:

- „Vermutlich denkt man aus purer Bequemlichkeit nicht über einen Wechsel seines Stromanbieters nach."

- „Ich konnte mich bislang einfach noch nicht dazu durchringen, mich mit der Auswahl eines neuen Stromanbieters zu beschäftigen."

- „Bei einem Umzug in eine andere Gegend würde ich wohl wieder zum dort ansässigen Versorger gehen, da mir die Suche nach Alternativen zu mühsam ist."

- „Solange ich meinen Strom problemlos bekomme, gibt es für mich keinen Anlass, über einen neuen Anbieter nachzudenken."

Beim ersten Indikator handelt es sich um eine projektive Aussage, mittels derer das Problem der sozialen Erwünschtheit umgangen werden soll. Inhaltlich versucht er, die Bequemlichkeit der Versuchspersonen zu messen, so dass die Ausführungen zum Bequemlichkeits-Indikator von HerRmann ET AL. (2000, S. 304) hier analog gelten. Der zweite Indikator lässt sich ohne Sinnverstellung verkürzen: „Ich habe mich noch nicht mit der Auswahl eines neuen Stromanbieters beschäftigt." Diese Kurzform verdeutlicht, dass im Grunde nichts anderes als ein Spezialfall des Noch-nicht-gewechselt-Habens gemessen wird. Der dritte Indikator misst zumindest zu einem großen Teil die „Mühsamkeit der Suche nach Alternativen“, die den Suchkosten zuzurechnen ist. Der vierte Indikator lässt sich zum besseren Verständnis ebenfalls verkürzen, da die Kondition („solange ich meinen Strom problemlos bekomme“) rein rhetorischer Natur ist. Es ist offensichtlich, dass sich 
auch die Indikatoren der beiden Operationalisierungen auf die genannten drei Kategorien aufteilen lassen.

Welche Konsequenzen ergeben sich nun aus den drei unterschiedlichen Kategorien von Trägheit speziell für das Wechselverhalten bei Versorgungsbeziehungen? Kann Trägheit eine Ursache für ein zurückhaltendes Wechselverhalten sein?

Versteht man unter Trägheit das zögerliche Verhalten der Konsumenten (also im Sinne der ersten Kategorie von Definitionen), und sieht Trägheit zugleich als Erklärung dafür an, müsste Trägheit eine Ursache von Trägheit sein. Trägheit kann aber nicht Ursache ihrer selbst sein, weil sie sonst logisch sich selbst vorausgehen müsste. Trägheit in diesem Sinne führt also zu einer Tautologie und ist daher für die Modellierung des Wechselverhaltens nicht geeignet.

Häufig meinen die Befürworter von Trägheit als Ursache für die geringe Zahl von Anbieterwechseln mit dem Begriff „Trägheit“ ein Nicht-Wechseln aufgrund wahrgenommener Wechselkosten (also im Sinne der zweiten Kategorie von Trägheitsdefinitionen). Hier ist auch das Phänomen der Bequemlichkeit einzuordnen, nämlich als ein durch die Mühsamkeit der Handlung erklärtes Nicht-Handeln. Um diesen Sachverhalt konzeptionell zu durchdringen, bietet es sich an, die Trägheit in zwei Konstrukte aufzuteilen: erstens die wahrgenommenen Wechselkosten und zweitens den Anbieterwechsel als die relevante Handlung, auf die die wahrgenommenen Wechselkosten einwirken.

Analog lässt sich bei der dritten Auffassung von Trägheit als Handeln unter fehlendem Involvement vorgehen. Auch hier ist eine konzeptionelle Entflechtung ratsam: Es ist sinnvoll, die Konstrukte „Involvement" und „Anbieterwechsel“ separat zu betrachten und in ein komplexeres Modell des Wechselverhaltens einzubringen.

Auf eine weitere Verwendung des Begriffs „Trägheit" wird in der vorliegenden Arbeit verzichtet. 


\section{Theoretische Grundlagen}

Dieses Kapitel stellt eine Reihe von Theorien vor, die potenziell einen Erklärungsbeitrag zum Wechselverhalten von Konsumenten bei Versorgungsbeziehungen zu leisten vermögen. Zunächst beschäftigt sich Abschnitt 3.1 mit Theorien und Konzepten, die erklären, warum Konsumenten bei ihrem Anbieter bleiben. Abschnitt 3.2 geht dann auf Theorien ein, aus denen sich Aussagen darüber ableiten lassen, warum Konsumenten ihren Anbieter wechseln.

\subsection{Theorien zur Fortsetzung von Versorgungsbeziehungen}

Dieser Abschnitt beschäftigt sich mit Theorien, die den Verbleib von Kunden in Versorgungsbeziehungen zu erklären vermögen. Unterabschnitt 3.1.1 stellt die Lerntheorie der instrumentellen Konditionierung vor. Gemäß dieser Theorie bestätigt Kundenzufriedenheit Konsumenten darin, bei ihrem Anbieter zu bleiben. Die Fortsetzung von Versorgungsbeziehungen wird dabei auf den Willen der Konsumenten zurückführt. Unterabschnitt 3.1.2 ergänzt diese Sichtweise um weitere Ursachen für den Fall, dass kein Wille zur Fortsetzung besteht.

\subsubsection{Die Lerntheorie der instrumentellen Konditionierung}

Die Lerntheorie der instrumentellen Konditionierung spielt eine wichtige Rolle in der Beziehungsmarketingforschung: Sie bietet einen Erklärungsansatz für loyales Kundenverhalten.

In einer weiten Definition versteht man unter Lernen „[...] the process by which experience leads to changes in knowledge, attitudes and/or behavior" (ENGEL ET AL., 1986, S. 426). Die menschlichen Lernprozesse gestalten sich vielschichtig und komplex, daher entwickelte sich eine Vielzahl an Theorien, die auf die Erklärung des Lernverhaltens von Individuen abzielen. Einen Überblick über diese Lerntheorien geben BowER und HILGARD (1984).

Als eine der traditionellen Lerntheorien zählt die Lerntheorie der instrumentellen Konditionierung zu den Stimulus-Response-Theorien. Ihnen ist gemein, dass sie auf eine expli- 
zite Modellierung der Vorgänge, die innerhalb des Organismus vor sich gehen, verzichten. Sie sehen den Menschen als eine „Black Box“, die Stimuli aus der Umwelt empfängt, verarbeitet und mit einer bestimmten Reaktion beantwortet.

Die Lerntheorie der instrumentellen Konditionierung ist insbesondere für die Analyse der Wirkbeziehung zwischen Kundenzufriedenheit und Wechselabsicht bzw. Wechselvollzug von Bedeutung. Sie besagt, dass Lernen nach dem Verstärkungsprinzip funktioniert, d. h. aufgrund der Konsequenzen bestimmter Verhaltensweisen erfolgt. Die Stimuli entstehen dabei durch Umweltreaktionen auf das eigene Verhalten. Man spricht auch von operanter Konditionierung (engl.: operant conditioning, vgl. Nord und Peter, 1980, S. 38). Die instrumentelle Konditionierung basiert auf dem „Law of Effect“ des Lernpsychologen EDWARd Thorndike (1874-1949) und wurde von dem Psychologen Frederic Skinner (1904-1990) weiterentwickelt (GIERING, 2000, S. 57).

Die Lerntheorie der instrumentellen Konditionierung differenziert zwischen positiver und negativer Verhaltensverstärkung. Während negative Verhaltensverstärker als Bestrafung von Verhalten zu Verhaltensänderungen führen, fungieren positive Verhaltensverstärker als Belohnung und bewirken, dass ein bestimmtes Verhalten wiederholt oder beibehalten wird (WILKIE, 1994, S. 270).

Assael (1992, S. 100) bezieht die Lerntheorie der instrumentellen Konditionierung insbesondere auf Konsumentenverhalten, das einerseits durch ein hohes Involvement und andererseits durch Gewohnheiten geprägt ist.

Die Lerntheorie der instrumentellen Konditionierung liefert einen Erklärungsbeitrag dafür, warum Kunden, die mit ihrem Anbieter zufrieden sind, diesen nicht wechseln: Wenn ein Kunde mit seinem Anbieter zufrieden ist, wirkt seine Zufriedenheit als eine Art positive Verhaltensverstärkung und erhöht somit die Wahrscheinlichkeit, dass der Kunde seinem Anbieter treu bleibt. Als theoretische Fundierung der Wirkung von Kundenzufriedenheit auf Wechselabsicht und Wechselvollzug hat die Lerntheorie der instrumentellen Konditionierung in der Marketingforschung bereits breite Anwendung gefunden (HomBurG ET AL., 1999, S. 179).

\subsubsection{Wille vs. Zwang zur Fortsetzung einer Geschäftsbeziehung}

Es gibt eine Vielzahl von Faktoren, die Kunden dazu veranlassen, eine Geschäftsbeziehung mit einem bestimmten Anbieter fortzusetzen. Sie lassen sich mit den Worten von JoHnsON (1982, S. 52) auf zwei grundlegende Ursachen zurückführen: „People stay in relationships for two major reasons: because they want to; and because they have to." Mit anderen Worten (EGGERT, 2003, S. 53): 
- „Kunden setzen eine Geschäftsbeziehung fort, weil sie es wollen.“

- „Kunden setzen eine Geschäftsbeziehung fort, weil sie es müssen.“

$\mathrm{Zu}$ ähnlichen Ergebnissen gelangt man auch anderenorts in der sozial- und wirtschaftswissenschaftlichen Literatur. Die sozialwissenschaftliche Literatur zu zwischenmenschlichen Beziehungen unterscheidet bspw. zwischen „dedication-based relationship maintenance" und "constraint-based relationship maintenance" (STANLEY und MARKMAN, 1992, S. 595). „Dedication“ steht für den Wunsch eines Individuums, die Qualität der Beziehung zum Nutzen und Wohle beider Kontrahenten zu pflegen oder zu verbessern. Unter „constraints“ sind Zwänge zu verstehen, die ein Individuum ungeachtet seines Willens dazu veranlassen, eine Beziehung beizubehalten.

Eine ähnliche Unterscheidung findet sich auch in der Literatur zur Mitarbeiterbindung: Ein Mitarbeiter kann bei einem Unternehmen verbleiben, weil er es bevorzugt oder weil er einen Wechsel des Arbeitsplatzes nicht als eine realistische Option ansieht (Iverson und Roy, 1994, S. 17). Im genannten Schrifttum herrscht Konsens darüber, dass beide Motivationen untersucht werden müssen, um ein umfassendes Verständnis dafür zu entwickeln, warum Beziehungen beibehalten werden. BENDAPUdi und BERRY (1997, S. 17) kritisieren, dass in der Marketingwissenschaft meist nur eine Motivation betrachtet wird, also entweder der Wunsch, eine Beziehung fortzusetzen, oder die Abhängigkeit innerhalb einer Beziehung.

Ganesan (1994, S. 1) regt an, sowohl den Wunsch zur Fortsetzung einer Beziehung als auch den Zwang zum Verbleib in einer Beziehung zu betrachten, um Beziehungen auf den Grund zu gehen. Morgan und Hunt (1994, S. 33) ziehen einen Vergleich zur Medizin: So wie zur Erfassung einer speziellen Pathologie gesunde und kranke Personen untersucht werden müssen, sollten sich zukünftige Forschungsarbeiten zur Kundenbindung sowohl mit positiven als auch negativen Motivationen zum Verbleib in einer Beziehung befassen, um so das Phänomen besser zu begreifen.

In Anlehnung an Bliemel und Eggert (1998a, S. 3; 1998b, S. 57) lassen sich die unterschiedlichen Gründe zur Fortsetzung einer Beziehung mit Hilfe der Dimensionen Wille zur Fortsetzung und Zwang zur Fortsetzung in einer 2×2-Matrix darstellen (vgl. Tab. 3.1). Die vier Felder der Matrix können als Szenarien aufgefasst werden, unter denen Beziehungen fortgesetzt werden. Die vier Felder sind wie folgt zu verstehen:

- Im ersten Szenario zwingen hohe Wechselbarrieren den Kunden zur Fortsetzung der Beziehung, auch wenn dieser die Beziehung nicht fortsetzen will. 


\section{Kunden setzen eine Beziehung fort, weil ...}

... sie es wollen.

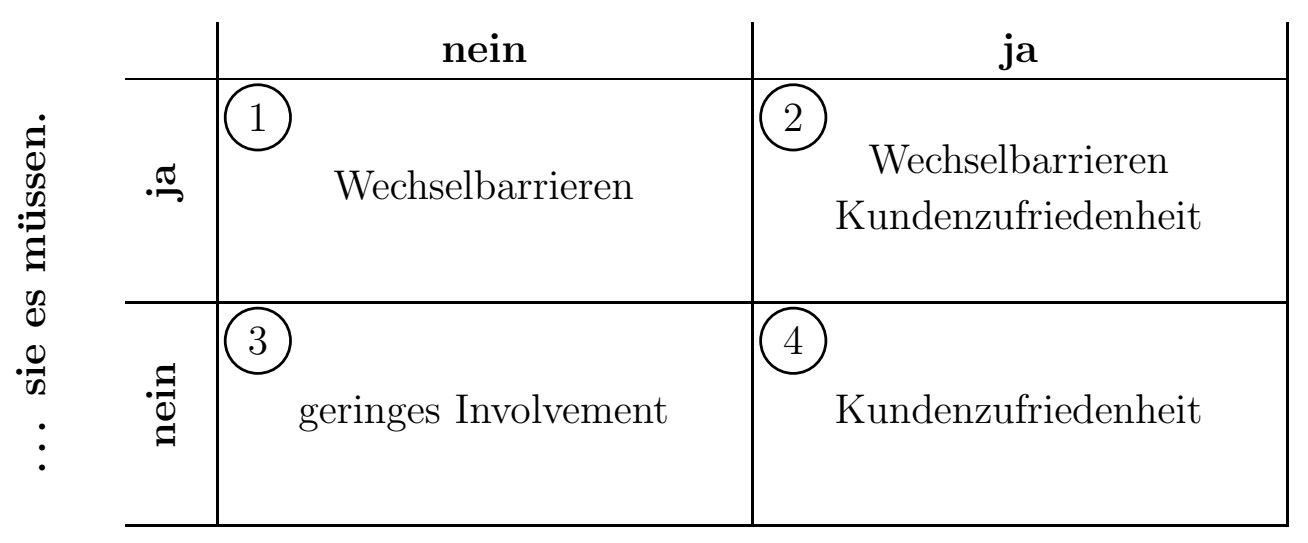

Tabelle 3.1: Warum Kunden ihre Geschäftsbeziehung fortsetzen. In Anlehnung an BLIEMEL und EGGERT (1998a, S. 3; 1998b, S. 57)

- Im zweiten Szenario will der Kunde die Beziehung fortsetzen, weil er mit dem Anbieter zufrieden ist. Zudem zwingen ihn hohe Wechselbarrieren zur Fortsetzung.

- Im dritten Szenario bestehen von Seiten des Kunden weder der Wille noch der Zwang zur Fortsetzung der Beziehung. Als Erklärung für die Fortsetzung eignet sich niedriges Involvement: Der Kunde ist nicht involviert genug, um einen Anbieterwechsel vorzunehmen.

- Im vierten Szenario ist der Kunde mit seinem Anbieter zufrieden und will daher gar nicht wechseln.

Das vorgestellte Konzept leistet einen wichtigen Beitrag zur Begründung, warum Versorgungsbeziehungen fortgesetzt werden. Ergänzend zur Lerntheorie der instrumentellen Konditionierung zeigt es auf, dass Versorgungsbeziehungen nicht nur willentlich, sondern auch basierend auf Zwang oder einem zu niedrigen Involvement fortgesetzt werden können.

\subsection{Theorien zum Anbieterwechsel bei Versorgungsbeziehungen}

Dieser Abschnitt beschäftigt sich mit Theorien, die nicht nur die Fortsetzung von Versorgungsbeziehungen, sondern auch deren Beendigung zu erklären vermögen. 
Unterabschnitt 3.2.1 stellt die Theorie der kognitiven Dissonanz vor. Gemäß dieser Theorie trachten Konsumenten danach, kognitive Dissonanzen zu vermeiden. Zufriedene Konsumenten bleiben demnach bei ihrem Anbieter, um ihr psychisches Gleichgewicht beizubehalten. Unzufriedene Konsumenten senken ihr Erwartungsniveau oder wechseln ihren Anbieter.

Die Theorie überlegten Handelns ist Thema von Unterabschnitt 3.2.2. Mit ihr lässt sich willentliches Verhalten, also auch Wechselverhalten, abbilden. Eine Weiterentwicklung der Theorie überlegten Handelns ist die Theorie des geplanten Verhaltens. Diese bildet den Inhalt von Unterabschnitt 3.2.3. Die Theorie des geplanten Verhaltens beschränkt sich nicht nur auf willentliches Verhalten, sondern erklärt auch das Zustandekommen anderer Verhaltensarten.

Zum Schluss des Kapitels wird in Unterabschnitt 3.2.4 die Austauschtheorie vorgestellt. Die Austauschtheorie führt die Fortsetzung oder Beendigung von Beziehungen auf die Erfüllung individueller Vergleichsstandards zurück. Als maßgebliche Determinanten des Wechselverhaltens lassen sich durch sie die Kundenzufriedenheit, die Attraktivität der Alternativen und die mit einem Anbieterwechsel verbundenen Wechselkosten identifizieren.

\subsubsection{Die Theorie der kognitiven Dissonanz}

Die Theorie der kognitiven Dissonanz wurde erstmals 1957 von Festinger aufgestellt. Sie gilt als die am besten erforschte Theorie der Sozialpsychologie (KREch, 1992, S. 52). Sie ist unter die verhaltenswissenschaftlichen Stimulus-Organism-Response-Theorien einzuordnen, „da sie ausgehend von einem bestimmten Auslöser oder Reiz die inneren Prozesse in einem Organismus (Wahrnehmen, Nachdenken etc.) abbildet und hierdurch die resultierende Reaktion des Organismus erklärt" (GIERING, 2000, S. 54).

Grundlage der Theorie der kognitiven Dissonanz ist die Annahme, dass Individuen ein dauerhaftes Gleichgewicht ihres kognitiven Systems anstreben. Unter einem kognitiven System sind die Summe von Wissen, Erfahrungen und Meinungen sowie die Beziehungen dazwischen zu verstehen. Dissonanz bezeichnet in diesem Kontext eine unangenehme psychologische Spannung, die dann empfunden wird, wenn eine Kognition konträr zu einer anderen ist (vgl. OshiKawa, 1968, S. 429). Überschreitet die kognitive Dissonanz eine individuell verschiedene Toleranzgrenze, versucht das Individuum, mittels eines geeigneten Verhaltens wieder in ein kognitives Gleichgewicht zu gelangen (KROEBER-RIEL und Weinberg, 2003, S. 182). Laut Loudon und Della Bitta (1993, S. 583f.) steigt die Wahrscheinlichkeit für das Entstehen von kognitiven Dissonanzen mit der Unwiderrufbarkeit der Entscheidung, der Attraktivität der nicht gewählten Alternativen, der Anzahl 
an Alternativen, der Varianz unter den Alternativen, der Bedeutung der Entscheidungssituation sowie geringerem Entscheidungsdruck.

Aus der Theorie der kognitiven Dissonanz lässt sich ableiten, dass Unzufriedenheit das Wechselverhalten fördert: Wenn der Konsument unzufrieden mit seiner gewählten Alternative ist, stellt er eine Diskrepanz zwischen erwarteter und wahrgenommener Leistung fest. Der Konsument wird versuchen, die hieraus entstehenden kognitiven Spannungen zu reduzieren - entweder durch einen Wechsel des Anbieters (PETER und Olson, 1994, S. 168) oder durch eine Absenkung des Anspruchsniveaus respektive der Erwartungen an die Leistung.

Des Weiteren lässt sich aufbauend auf der Theorie der kognitiven Dissonanz ein Zusammenhang zwischen Kundenzufriedenheit und treuem Kundenverhalten wie folgt konstruieren (WeinBerg, 1987, S. 165): Ein Kunde, der mit seinem Anbieter zufrieden ist, befindet sich diesbezüglich in einem psychischen Gleichgewicht. Indem er seine Geschäftsbeziehung beibehält und sich insofern loyal verhält, vermeidet er kognitive Dissonanzen. Die Theorie der kognitiven Dissonanz zeigt damit auf, dass Kundenzufriedenheit dafür entscheidend ist, wann Kunden bei ihrem Anbieter bleiben wollen.

Als theoretische Fundierung der Wirkung von Kundenzufriedenheit auf Wechselabsicht und Wechselvollzug hat die Theorie der kognitiven Dissonanz in der Marketingforschung bereits breite Anwendung gefunden (Homburg ET AL., 1999, S. 179).

\subsubsection{Die Theorie überlegten Handelns}

Die Theorie überlegten Handelns (engl.: Theory of Reasoned Action) geht auf FisHBEIN und AJzen (1975) zurück. Das zentrale Element der Theorie überlegten Handelns ist die These, dass menschliches Verhalten vor allem auf die Absicht eines Individuums zurückzuführen ist, eben dieses Verhalten an den Tag zu legen. Einstellungen beeinflussen das Verhalten nicht unmittelbar, sondern nur über die Verhaltensabsichten, d.h. Entscheidungen, auf eine bestimmte Art und Weise zu handeln. Die Verhaltensabsicht mediiert sozusagen den Einfluss von Einstellungen auf das Verhalten.

Durch die Einbindung des Konstrukts Verhaltensabsicht lösen FishBeIN und AJZEN einerseits die Frage, auf welche Weise der Geistesvorgang „Besitzen einer bestimmten Einstellung" letztendlich in beobachtbares Verhalten transformiert wird. Andererseits beschränkt sich die Aussagekraft der Theorie auf willentliches Verhalten, d.h. Individuen verhalten sich auf eine bestimmte Weise, weil sie sich dazu entschlossen haben.

Die Theorie überlegten Handelns sieht zwei Determinanten für die Verhaltensabsicht vor: Einstellungen und subjektive Normen. Über die Art der Einstellungen gibt es unterschied- 
liche Auffassungen. Ajzen und Fishbein (1980, S. 54) weisen darauf hin, dass sie ihre Theorie des überlegten Handelns auf die Einstellung gegenüber der betreffenden Handlung und nicht auf die Einstellung gegenüber einem Objekt anwenden. Neuere Arbeiten (vgl. z. B. Gotlieb ET AL., 1994, S. 876) sehen jedoch eine Erweiterung der Theorie überlegten Handelns um die Einstellung gegenüber einem Objekt als zulässig an. Den Einstellungen vorgelagert sind die sog. „beliefs“ als Eindrücke eines Individuums von einem Sachverhalt. Des Weiteren wird die Verhaltensabsicht durch subjektive Normen determiniert. Subjektive Normen spiegeln wider, inwieweit ein Individuum glaubt, dass relevante Andere denken, dass es sich auf eine bestimmte Weise verhalten solle. Unter relevanten Anderen sind Personen zu verstehen, deren Meinung über sein Verhalten dem Individuum wichtig ist. Einige Forscher stellen jedoch in Frage, ob die subjektive Norm als eigenständiges Konstrukt zu behandeln ist, da sie konzeptionelle Überschneidungen mit dem Konstrukt Verhaltenseinstellung aufweist (vgl. bspw. Oliver und BeARden, 1985, S. 324f.; Warshaw, 1980a, S. 158). Miniard und Cohen (1979, S. 103) machen darauf aufmerksam, dass diese Überschneidungen eine doppelte Erfassung bestimmter Einflussfaktoren implizieren können. Darüber hinaus zeigen manche empirischen Studien, dass sich die beiden Konstrukte Verhaltenseinstellung und subjektive Norm nicht empirisch unterscheiden lassen (RYAN, 1978).

Abb. 3.1 stellt schematisch den Aufbau der Theorie überlegten Handelns dar. Sie verdeutlicht die mediierende Rolle der Verhaltensabsicht: Einstellung und subjektive Norm wirken nicht direkt auf das Verhalten, sondern nur indirekt über die Verhaltensabsicht.

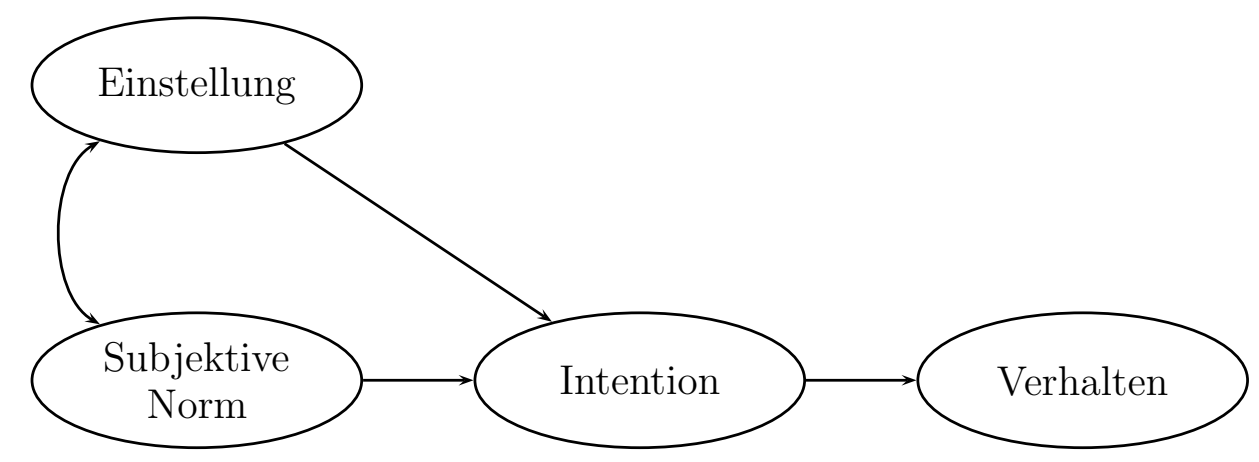

Abbildung 3.1: Kernmodell der Theorie des überlegten Handelns. Angelehnt an FisHBEIN (1980, S. 69) 
Trotz der durchaus zahlreichen Ansatzpunkte für Kritik bescheinigen SHEPPARD ET AL. (1988, S. 388ff.) - als Ergebnis einer Meta-Analyse über empirische Arbeiten zur Theorie überlegten Handelns - dem Modell von Fishbein und AJzen eine hohe Prognosegüte.

Die Theorie überlegten Handelns wurde bereits mehrfach zur Erklärung von Kundenloyalität adaptiert. Hierbei ist zu beachten, dass dem Konzept Kundenloyalität unterschiedliche Konzeptualisierungen und Operationalisierungen zu Grunde liegen. Marketingforscher haben sowohl einstellungs- als auch verhaltensorientierte Sichtweisen bei der Definition und der Untersuchung der Kundenloyalität eingenommen (vgl. Oliver, 1999; ZeithamL, 2000). Aus einstellungsorientierter Perpektive lässt sich Kundenloyalität auffassen als das spezifische Verlangen eines Kunden, eine Geschäftsbeziehung mit einem (Dienstleistungs-) Anbieter fortzuführen (Czepiel und Gilmore, 1987, S. 91). Aus verhaltensorientierter Perspektive wird unter Kundenloyalität Wiederholungskaufverhalten verstanden, das sich im Anteil eines bestimmten Produkts an den Gesamtkäufen eines Kunden innerhalb einer Produktkategorie manifestiert (NeAL, 1999, S. 21). YAng und Peterson (2004, S. 802) merken hierzu an, dass "[t]he dilemma lies in the fact that intention may not lead to action, and repeated buying behavior may not reflect intentions."

Die Theorie überlegten Handelns stößt an Grenzen, wenn das Verhalten nicht allein willensgesteuert erfolgt oder wenn das Verhalten nur zum Teil dem menschlichen Willen unterliegt. Will ein Paar bspw. innerhalb der nächsten drei Jahre ein Kind bekommen, hängt die Realisierung nicht allein von der entsprechenden Absicht ab. In einem viel beachteten Artikel kritisierte LiskA (1984, S. 63) die Beschränkung der Theorie geplanten Handelns auf gänzlich dem Willen unterliegende Handlungen, weil dadurch nicht nur habituelles Verhalten, sondern jegliches Verhalten, das „skills, abilities, opportunities and the cooperation of others" erfordert, ausschließt. Die Fortsetzung von Beziehungen lässt sich also insbesondere dann nicht durch die Theorie überlegten Handelns abbilden, wenn die Fortsetzung auf Zwängen beruht (EGGERT, 1999, S. 68).

\subsubsection{Die Theorie des geplanten Verhaltens}

Als Reaktion auf die Restriktionen der Theorie überlegten Handelns und insbesondere die Kritik von Liska (1984) entstand die Theorie des geplanten Verhaltens. AJzen (1985) stellte die Theorie des geplanten Verhaltens (engl.: Theory of Planned Behavior) auf, indem er die Theorie überlegten Handelns um eine zusätzliche Variable erweiterte: die wahrgenommene Verhaltenskontrolle. Sie dient vor allem zur besseren Vorhersage von Verhaltensabsicht und wirklichem Verhalten (siehe auch AJZEn und MAdDEn, 1986). In der Theorie des geplanten Verhaltens wird die wahrgenommene Verhaltenskontrolle definiert als die Wahrnehmung einer Person, wie einfach oder wie schwer es ihr fällt, ein 
bestimmtes Verhalten auszuführen (EAGLY und Chaiken, 1993, S. 186). Mit Hilfe dieses neuen Konstrukts überwindet die Theorie des geplanten Verhaltens die Randbedingung der Theorie überlegten Handelns, die in einer vollständigen Kontrolle durch den Willen besteht (MADDEN ET AL., 1992, S. 4).

Abb. 3.2 stellt das Kernmodell der Theorie des geplanten Verhaltens schematisch dar. Im Vergleich mit Abb. 3.1 werden die beiden neu hinzugekommenen Hypothesen deutlich: Je mehr Möglichkeiten und Ressourcen ein Individuum zu besitzen glaubt bzw. je weniger hindernisreich oder mühsam ein Individuum eine Handlung einschätzt, desto eher entwickelt es erstens die Absicht, eine bestimmte Handlung auszuführen, und desto eher führt es zweitens eine bestimmte Handlung tatsächlich durch. Des Weiteren ist festzuhalten, dass zahlreiche kausale Effekte nicht nur in eine Richtung wirken; insbesondere die Intentionsantezedenzien wirken auch wechselseitig aufeinander ein (AJZEN, 1989, S. 248).

Durch die Theorie des geplanten Verhaltens lässt sich im Gegensatz zur Theorie überlegten Handelns auch der Zwang zur Fortsetzung einer Versorgungsbeziehung abbilden. Die Theorie des geplanten Verhaltens bietet mit dem Konstrukt der Verhaltenskontrolle das Handwerkszeug, ökonomische oder vertragliche Wechselhemmnisse, die bislang nur unzureichende Berücksichtigung in der Kundenbeziehungsliteratur fanden (OEvERMAnN, 1996, S. 9), in die Verhaltensanalyse miteinzubeziehen.

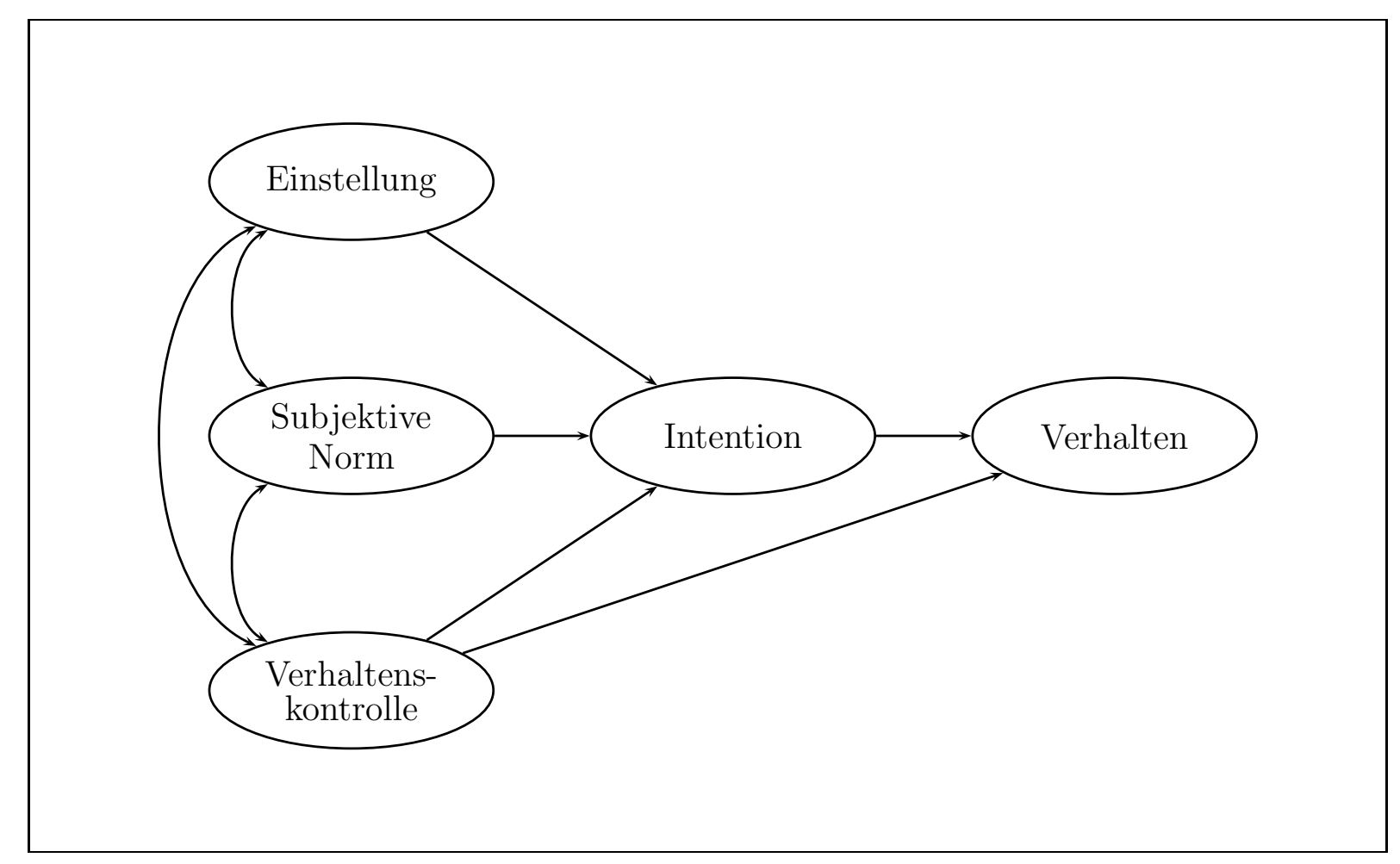

Abbildung 3.2: Kernmodell der Theorie des geplanten Verhaltens. Angelehnt an AJZEN (1985, S. 33; 1989, S. 252) 
Die Theorie des geplanten Verhaltens ist zwar nur eingeschränkt zur Erklärung der Fortsetzung von Versorgungsbeziehungen geeignet, schließlich müssen Konsumenten bei Versorgungsbeziehungen nicht explizit eine Absicht zum Bleiben bilden. Anders verhält es sich jedoch mit dem Wechselverhalten: Um einen Anbieter zu wechseln, bedarf es einer Absicht dazu, die sich spätestens in der Unterschrift unter einen neuen Vertrag manifestiert. Selbst wenn ein Anbieterwechsel durch ein Haustürgeschäft oder eine andere aggressive Vertriebsform zu Stande gekommen sein sollte, kann dem Konsumenten nicht der Wille abgesprochen werden, den Anbieter zu wechseln. Zudem basiert jedwedes dem freien Willen unterliegende Verhalten auf Verhaltensabsichten (PETER und Olson, 1999, S. 407).

BANSAL und TAYLOR (1999, S. 201) sehen in den wahrgenommenen Wechselkosten eine spezielle Form von Verhaltenskontrolle. Gerade das Konstrukt der wahrgenommenen Verhaltenskontrolle verdient besondere Beachtung: Da es sowohl auf die Verhaltensabsicht als auch auf das Verhalten wirkt, besitzt es das Potenzial, die Diskrepanz zwischen Wechselabsicht und Wechselverhalten zumindest ansatzweise zu erklären.

\subsubsection{Die Austauschtheorie}

Die Austauschtheorie (auch bezeichnet als „soziale Austauschtheorie“) vermag wie kaum eine andere interpersonelle Theorie zu erklären, wieso und wann Beziehungen begonnen, aufrechterhalten und beendet werden. Sie geht zurück auf die Arbeiten von Homans (1958, 1961) sowie Thibaut und Kelley (1959). Gegenstand der Analyse ist das Interaktionsverhaltens auf der individuellen Ebene, wenn es um den Austausch von Werten geht (BAgozzi, 1975; Houston und Gassenheimer, 1987).

Ursprünglich diente die Austauschtheorie allgemein zur Erklärung von sozialen Interaktionen. Später richtete sich das Augenmerk auch spezieller auf das Interaktionsverhalten von Anbieter und Kunde: Im Business-to-Business-Marketing schlugen DwYER ET AL. (1987) und Hallén ET AL. (1991) die Austauschtheorie zur Modellierung von Anbieter-Nachfrager-Beziehungen vor; Anderson und NARUs $(1984,1990)$ wendeten sie auf Marktbeziehungen an. Auch in der Konsumentenverhaltensforschung wird die Austauschtheorie zur Analyse von Anbieter-Nachfrager-Beziehungen herangezogen (vgl. z. B. Ganesh et AL., 2000; Brinberg und Wood, 1983). Weitere Anwendungsfelder der Austauschtheorie in der Marketingforschung sind bspw. die Verbreitung von Innovationen (Leung, 2001), die Analyse der Lebensqualität von Bewohnern im Rahmen des Tourismusmarketing (PERDUE ET AL., 1999), das Spenden an Wohltätigkeitsorganisationen (MAthur, 1996) oder der sog. „Service Performance Gap“ im Dienstleistungsmarketing (Chenet et AL., 1999, 2000). Die Austauschtheorie bildet eine Schnittstelle zwischen 
verhaltenswissenschaftlicher und ökonomischer Forschung. Als sozialpsychologische Theorie wird die Austauschtheorie dem neobehavioristischen Paradigma zugerechnet (BRUHN, 2002, S. 18).

In einer qualitativen Untersuchung gelangen SorCE und EDWARDS (2004) zu dem Ergebnis, dass Konsumenten die Konstrukte der Austauschtheorie als wichtige Bestandteile von Kundenbeziehungen erachten.

Vereinfacht gesprochen besagt die Austauschtheorie, dass Menschen danach trachten, Beziehungen zu entwickeln, innerhalb derer Nutzen maximiert wird. Nutzen ist hierbei als Nettonutzen aufzufassen, d.h. er bildet die Differenz aus Belohnungen und Kosten, die aus einer Beziehung resultieren. Diese Betrachtungsweise legt nahe, dass Beziehungen gewöhnlich so lange unterhalten werden, wie die Belohnungen die Kosten übersteigen, und anderenfalls beendet werden.

Die Austauschtheorie geht von der Annahme aus, dass Interaktion aus dem Empfangen und Abgeben physischer und psychischer Ressourcen besteht und sich als Austausch von Belohnungen und Kosten ansehen lässt: Belohnungen umfassen Freude, Zufriedenstellung oder Gratifikation, durch die Bedürfnisse gestillt oder Wünsche erfüllt werden. Unter Kosten sind solche Faktoren zu verstehen, die einer gewünschten Verhaltensabfolge im Wege stehen oder die Erfüllung von Wünschen verhindern. ThiBaut und Kelley (1959, S. 12f.) erklären: „Thus cost is high when great physical or mental effort is required, when embarrassment or anxiety accompany the action, or when there are conflicting forces or competing response tendencies of any sort."

Die Austauschtheorie ist kontextfrei (RodríGuez und Wilson, 2002, S. 54), d. h. sie trifft keine Annahmen über das, was die Akteure bewerten. Dementsprechend verhalten sich Akteure selbst dann ähnlich, wenn es um so unterschiedliche Dinge wie Geld oder soziale Wertschätzung geht (Molm, 1994). Die via Beziehungen ausgetauschten Objekte können vielerlei Gestalt haben: Ebenso wie Güter, Dienstleistungen und Geld können auch Status, Information, Freundschaft, soziale Akzeptanz, Sicherheit oder Liebe als Austauschobjekt dienen (KNAPP, 1984).

Die Austauschtheorie stellt ein ökonomisches Modell von Beziehungen dar. Es besagt, dass jede Person in einer Beziehung gewisse Erwartungen hat und einen bestimmten Maßstab an das Verhalten ihres Partners anlegt. Menschen gehen Beziehungen mit einer Vorstellung darüber ein, welche Art von Belohnung und Nutzen realistischerweise aus der Beziehung gezogen werden kann. Sie bilden einen Vergleichsstandard (Comparison Level: $C L)$, der sich durch frühere Ergebnisse sowie durch soziale Vergleichsprozesse bestimmt. Sofern dieser Vergleichsstandard erreicht oder gar übertroffen wird, ergibt sich Zufriedenheit mit dem Partner und der Beziehung. In diesen Fällen gleichen die wahrgenommenen 
Belohnungen die wahrgenommenen Kosten aus. Sowohl für die Qualität als auch die Dauerhaftigkeit der Beziehung ist es von Bedeutung, dass die Erwartungen an die Beziehung (über-)erfüllt werden. Dabei spielen nicht nur die gegenwärtigen Belohnungen und Kosten eine Rolle. Menschen antizipieren auch die zukünftigen Belohnungen und Kosten. Aus Studien von Taylor und Altman (1973) gelangt Littlejohn (1996, S. 264) zu der Erkenntnis, dass „relational partners not only assess the rewards and costs of the relationship at a given moment but also use the information they have gathered to predict the rewards and costs in the future."

Wie viele Kosten im Verhältnis zu den Belohnungen eine Person akzeptiert, ohne dass sie die Beziehung beendet, besagt ein weiterer Vergleichsstandard: der individuelle Vergleichsstandard für Alternativen $\left(C L_{\text {alt }}\right)$. Der Vergleichsstandard für Alternativen hängt hauptsächlich von der Attraktivität der unmittelbar verfügbaren Alternativen ab (Kelley und Thibaut, 1978, S. 9). Wenn einer Person gleich attraktive oder attraktivere Konkurrenzangebote vorliegen, ist die Toleranz gegenüber nicht zufriedenstellender Leistung geringer, d. h. die Person wechselt eher den Anbieter.

Die Austauschtheorie besagt, dass Menschen die Ergebnisse aus einer Beziehung als die Differenz aus den in der Beziehung erfahrenen Belohnungen und Kosten bewerten. Die Bewertung einer Beziehung kann sich im Zeitablauf verändern. Wie Abb. 3.3 illustriert, gibt es vier mögliche Szenerien für die Bewertung einer Beziehung:

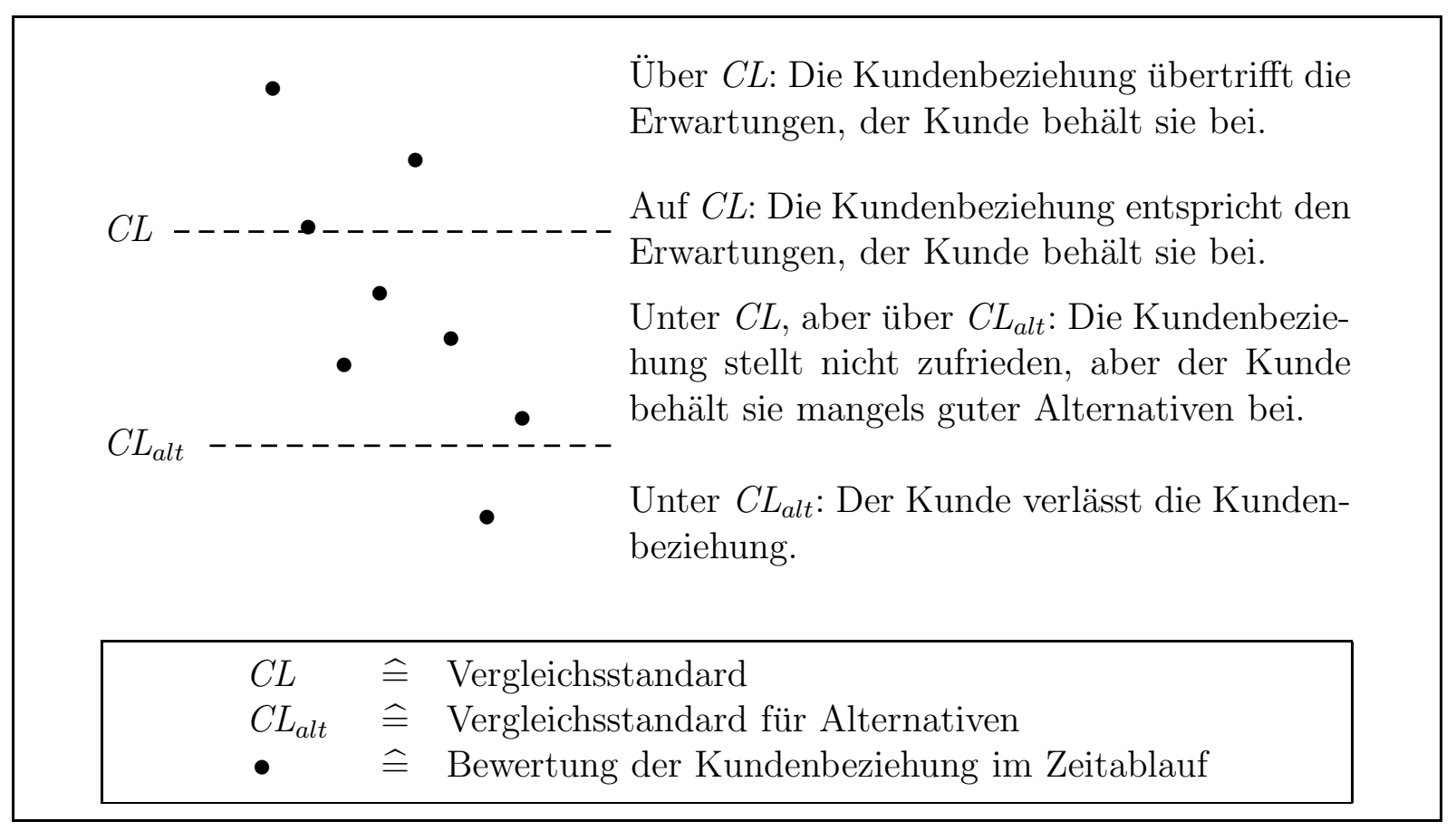

Abbildung 3.3: Ein Modell zur Austauschtheorie 
- Der Kunde bewertet die Beziehung als über dem Vergleichsstandard $C L$ liegend: Die Beziehung übertrifft dann die Erwartungen des Kunden, und der Kunde behält sie bei.

- Der Kunde bewertet die Beziehung als genau dem Vergleichsstandard $C L$ entsprechend: Die Kundenbeziehung entspricht dann den Erwartungen, und der Kunde behält sie bei.

- Der Kunde bewertet die Beziehung als zwischen dem Vergleichsstandard $C L$ und dem Vergleichsstandard für Alternativen $C L_{\text {alt }}$ liegend: Die Kundenbeziehung entspricht dann zwar nicht den Erwartungen, doch der Kunde behält sie bei, weil sich ein Anbieterwechsel nicht lohnt.

- Der Kunde bewertet die Beziehung als unter dem Vergleichsstandard für Alternativen $C L_{\text {alt }}$ liegend: Die Kundenbeziehung entspricht dann nicht den Erwartungen, und es lohnt sich, zu einem alternativen Anbieter zu wechseln. Der Kunde beendet daher die Beziehung.

Nach der Austauschtheorie können Kunden sich in Situationen befinden, in denen sie Geschäftsbeziehungen trotz geringer Belohnung aufrecht erhalten, nur weil die Kosten in Verbindung mit dem Wechsel zu einem anderen Anbieter zu hoch sind (ANDERSEN, 2002, S. 93; Kelley und Thibaut, 1978, S. 8f.). 


\section{Determinanten des Wechselverhaltens}

Welche Faktoren bestimmen, ob Konsumenten ihren Stromanbieter wechseln oder nicht? Diese Faktoren zusammenzustellen ist der Inhalt dieses Kapitels.

Kunden wollen eine Geschäftsbeziehung fortsetzen, wenn sie einen hohen Nettonutzen aus ihr erhalten. Der Nettonutzen kann dabei einerseits absolut sein, d. h. die Erwartungen an einen Anbieter werden erfüllt bzw. übererfüllt. Andererseits kann der Nettonutzen auch relativ sein, d.h. der derzeitige Anbieter erfüllt die Erwartungen besser als Konkurrenzanbieter. Ein zentrales Konstrukt, das sowohl die Erwartungen als auch den erhaltenen Nutzen von Kunden umfasst, ist die Kundenzufriedenheit. Mit dem Konstrukt Kundenzufriedenheit und seinem Einfluss auf die Wechselabsicht und den Wechselvollzug beschäftigt sich der Abschnitt 4.1.

Die Austauschtheorie besagt, dass die Beendigung von Beziehungen von der Attraktivität der Alternativen abhängt. Schließlich lässt sich durch einen Anbieterwechsel nur dann ein Wechselgewinn erzielen, wenn attraktive Alternativen zum bisherigen Anbieter verfügbar sind. Abschnitt 4.2 konzeptualisiert die Attraktivität der Alternativen und stellt Hypothesen zu ihrem Einfluss auf die Wechselabsicht und den Wechselvollzug auf.

Lange Zeit konzentrierte sich sowohl die wissenschaftliche Forschung als auch die Praxis bei den Wirkzusammenhängen im Kontext von Wechselverhalten und Kundenbindung auf den Begriff der Kundenzufriedenheit. So konnte z. B. zu Beginn der 1990er Jahre in Amerika und Europa eine starke Zunahme von Studien zum Thema Kundenzufriedenheit beobachtet werden (OLIVER, 1999, S. 33). Mittlerweile häufen sich jedoch Aussagen wie „a satisfied customer isn't enough“ (STEWART, 1997, S. 112) und „satisfying customers that have the freedom to make choices is not enough to keep them loyal" (Jones und SAsser, 1995, S. 91). Dies konnte auch durch eine Studie in der Automobilbranche bestätigt werden, die feststellte, dass zwar 85 bis $95 \%$ der Kunden Zufriedenheit bekunden, jedoch nur 30 bis $40 \%$ zu ihrem vorherigen Anbieter zurückkehren (OLIVER, 1999, S. 33). Während Kundenzufriedenheit hauptsächlich auf das „Nicht-Wechseln-Wollen“ abzielt, können Wechselbarrieren bzw. durch sie induzierte Wechselkosten erklären, inwiefern Konsumenten daran gehindert werden, ihren Anbieter zu wechseln. Wechselkosten sollten Kunden tendenziell dazu bewegen, eine Geschäftsbeziehung fortzusetzen. Mit Wechselbarrieren respektive wahrgenommenen Wechselkosten beschäftigt sich Abschnitt 4.3. 
Bisweilen setzen Kunden auch dann eine Geschäftsbeziehung fort, wenn weder ein Nettonutzen aus ihr erwächst, noch Wechselbarrieren bestehen. Als mögliche Ursachen für dieses Phänomen wird in Abschnitt 4.4 geringes Involvement diskutiert.

\subsection{Kundenzufriedenheit als Determinante des Wechselverhaltens}

Seit nahezu drei Jahrzehnten steht das Konzept der Kundenzufriedenheit an zentraler Stelle in der Marketingtheorie und -praxis. Dies bezeugen beispielhaft die viel zitierten Aufsätze von Anderson und Sullivan (1993), Fornell (1992), Fournier und Mick (1999) sowie Szymanski und Henard (2001). Allein in den 1970er und 1980er Jahren wurden mehr als 15.000 Artikel zum Thema Kundenzufriedenheit verfasst (PETERSON und Wilson, 1992, S. 61). Schon traditionell stellt das Marketing die Kundenzufriedenheit in den Mittelpunkt seines konzeptionellen Selbstverständnisses als marktorientierte Unternehmensführung (HERRMANN ET AL., 2000, S. 293), denn „[l]etztendlich trifft Kundenzufriedenheit den Kern des Marketing“ (Simon und Homburg, 1997, S. 19). Oliver (1997, S. 9) erachtet „[... ] generating customer satisfaction as the key to satisfying organizational goals [...]“. Peterson und Wilson (1992, S. 61) stellen fest, dass es aus einer normativen oder philosophischen Perspektive nicht möglich ist, gegen das Ziel Kundenzufriedenheit zu argumentieren. Sie schränken jedoch zugleich ein, dass Kundenzufriedenheit mit einem Profit für Unternehmen einhergehen sollte. Die gleiche Einschränkung thematisieren Simon und Homburg (1997, S. 19), indem sie anmerken, dass, wie alle anderen Aktivitäten des Unternehmens auch, das Streben nach Kundenzufriedenheit dem ökonomischen Imperativ zu unterwerfen sei - d. h. sie müsse mehr bringen als sie koste. Als wie bedeutsam die Kundenzufriedenheit angesehen wird, zeigt die Implementierung von sog. „Customer Satisfaction Indices“ in vielen europäischen Staaten, in Japan und in den USA (FORNELL, 1992, S. 6).

Die große Anzahl von Arbeiten, die sich mit Kundenzufriedenheit befassten, brachte auch eine Vielzahl unterschiedlicher Auffassungen des Konstrukts Kundenzufriedenheit mit sich. Aufbauend auf den vorherrschenden Auffassungen von Kundenzufriedenheit legt der Unterabschnitt 4.1.1 fest, was in der vorliegenden Arbeit unter Kundenzufriedenheit verstanden wird. Möglicherweise hat die Kundenzufriedenheit einen großen, wenn nicht gar den größten Einfluss auf das Wechselverhalten von Kunden. Die Rolle der Kundenzufriedenheit innerhalb des Wechselverhaltens von Konsumenten bei Versorgungsbeziehungen thematisiert der Unterabschnitt 4.1.2 anhand von Hypothesen zur Wirkung der Kundenzufriedenheit. 


\subsubsection{Das Konstrukt Kundenzufriedenheit}

Zur Kundenzufriedenheit gibt es eine Vielzahl von konkurrierenden Definitionen, Konzepten und Beschreibungen, doch fehlte es lange Zeit in der Forschung an einer allgemein anerkannten zugrunde liegenden Theorie (HAusknecht, 1990, S. 1). Inzwischen besteht weitgehende Einigkeit darin, dass Kundenzufriedenheit das Resultat eines psychischen Vergleichsprozesses darstellt. Das Zufriedenheitsurteil basiert auf mindestens zwei Stimuli: dem wahrgenommenen Nutzen des Konsums und einem Vergleichsmaß. Der am weitesten verbreitete Vergleichsstandard sind auf die Leistung bezogene Erwartungen. Diesen Vergleichsstandard verkörpert das u. a. von Oliver (1980), Boulding ET AL. (1993), Schütze (1992) sowie YI (1990) vertretene Disconfirmation-Paradigma, nach dem das Zufriedenheitsurteil das Ergebnis eines unbewusst erfolgenden Vergleichs zwischen der auf die Leistung bezogenen Erwartung und der tatsächlichen Erfahrung ist. Zusätzlich zum Vergleichsmaßstab Erwartungen nennen FourNIER und Mick (1999, S. 5f.) drei weitere Vergleichsstandards, die in der Literatur große Beachtung gefunden haben:

- Der Ansatz von Westbrook und Reilly (1983) verwendet Werte (bzw. Bedürfnisse, Wünsche) als Vergleichsmaßstab.

- Oliver und Swan (1989) schlagen „equity expectations“ vor, die Erwartung einer fairen Behandlung.

- Auf Cadotte et AL. (1987) geht der Vergleichsstandard der „experience-based norms" zurück.

Stauss (1999, S. 6) sieht es als gerechtfertigt an, sich auf das Disconfirmation-Paradigma zu beschränken, denn „,[z] um einen spielt es in der wissenschaftlichen Diskussion die weitaus bedeutendste Rolle, zum zweiten sind die anderen verhaltenswissenschaftlichen Konzepte in diesen Rahmen zu integrieren."

Die Gegenüberstellung der (nach dem Kauf) wahrgenommenen Produktleistung auf der einen Seite und der (vor dem Kauf) erwarteten Leistung auf der anderen Seite kann zu drei möglichen Kognitionen führen (Kotler und Bliemel, 2001, S. 362):

- Zufriedenheit, wenn die erhaltene Leistung genau den Erwartungen entspricht,

- Begeisterung, wenn die erhaltene Leistung die Erwartungen übertrifft, und

- Unzufriedenheit, wenn die erhaltene Leistung hinter den Erwartungen zurückbleibt. 
Kundenzufriedenheit kann sich auf zwei unterschiedliche Arten von Objekten beziehen: zum einen auf eine spezifische Transaktion oder ein Produkt, zum anderen kumulativ auf eine Geschäftsbeziehung mit einem Anbieter bzw. auf den Anbieter selbst. Die wiederholten Erfahrungen eines Kunden mit einem Anbieter und eine stets erneute Zufriedenheit mit der Leistung prägen und stabilisieren zukünftiges Verhalten, begründen also somit Kundenbindung (vgl. u. a. Anderson und Fornell, 1994, S. 254; Rust et Al., 1995, S. 64). Anderson Et AL. (1994, S. 54) stellen die These auf, dass „, [..] whereas transaction-specific satisfaction may provide diagnostic information about a particular product or service encounter, cumulative satisfaction is a more fundamental indicator of a firm's past, current and future performance." RUsT ET AL. (1995, S. 64) empfehlen, dass wenn „the [..] primary measurement objective is to predict behavior, then cumulative questions may be preferred."

Uneinigkeit herrscht in der marketingwissenschaftlichen Literatur darüber, ob es sich bei Kundenzufriedenheit um eine Einstellung handelt oder nicht. Während einige Autoren die Zufriedenheit ausschließlich als Ergebnis eines kognitiven Soll-Ist-Vergleichs konzeptualisieren (vgl. z. B. Giering, 2000, S. 14; Oliver, 1997, S. 13), verstehen andere Autoren die Kundenzufriedenheit als Einstellung (vgl. z. B. Churchill und Suprenant, 1982, S. 493; Westbrook und Reilly, 1983, S. 256). Bitner (1990, S. 70) erachtet als zentralen Unterschied zwischen Einstellungen und Kundenzufriedenheit, dass „, [.. ] satisfaction assessments relate to individual transactions whereas attitudes are more general." Dieser Unterschied löst sich auf, wenn man an Stelle der einzelnen Transaktionen die kumulative Kundenzufriedenheit betrachtet. In letzterem Fall lässt sich Kundenzufriedenheit als Einstellung auffassen.

Kundenzufriedenheit kann aus verschiedenen Komponenten bestehen. Speziell für den Strommarkt fordern Beutin und Werner (1999, S. 554), die Zufriedenheit der Kunden mit einzelnen Leistungsbestandteilen zu analysieren. Als Leistungsbestandteile betrachten sie die Berührungspunkte zwischen dem Unternehmen und seinen Kunden. Mit Hilfe qualitativer Interviews ermittelten sie sieben Leistungsbestandteile, die die Kundenzufriedenheit von Gewerbekunden mit einem Energieversorgungsunternehmen beeinflussen:

- Zufriedenheit mit dem Beschwerdemanagement,

- Zufriedenheit mit der Erstellung von Angeboten,

- Zufriedenheit mit der Rechnungsstellung,

- Zufriedenheit mit der Zusammenarbeit,

- Zufriedenheit mit dem Kundenzentrum, 
- Zufriedenheit mit den Dienstleistungen und

- Zufriedenheit mit der Energieversorgung.

Die relevanten Leistungsbestandteile im Haushaltskundenbereich unterscheiden sich leicht von denen im Gewerbekundenbereich. Da für Haushaltskunden gewöhnlich keine individuellen Angebote erstellt werden, entfällt der entsprechende Bestandteil. Dafür legen Haushaltskunden möglicherweise Wert auf ein lokales Engagement des Versorgers. Abb. 4.1 illustriert, aus welchen Teilkomponenten sich die Zufriedenheit von Haushaltsstromkunden mit ihren Energieversorgern zusammensetzen kann. Während die Analyse der einzelnen Kundenzufriedenheitskomponenten für das Marketingmanagement eine wertvolle Entscheidungsunterstützung bilden kann, liefert sie aufgrund der geringen Verallgemeinerbarkeit einen eher geringen Beitrag für die Marketingforschung.

Die Unterteilung der Kundenzufriedenheit in einzelne Komponenten ist inhaltlich identisch mit der zufriedenheitsorientierten Konzeptualisierung von Dienstleistungsqualität (vgl. hierzu Hentschel, 2000, S. 301-303). Ob ein Kunde seine Erwartungen als erfüllt ansieht und somit mit dem Produkt des Anbieters zufrieden ist oder nicht, hängt insbesondere von der wahrgenommenen Produktqualität ab (Vollmer, 2002, S. 19). Hinter dem

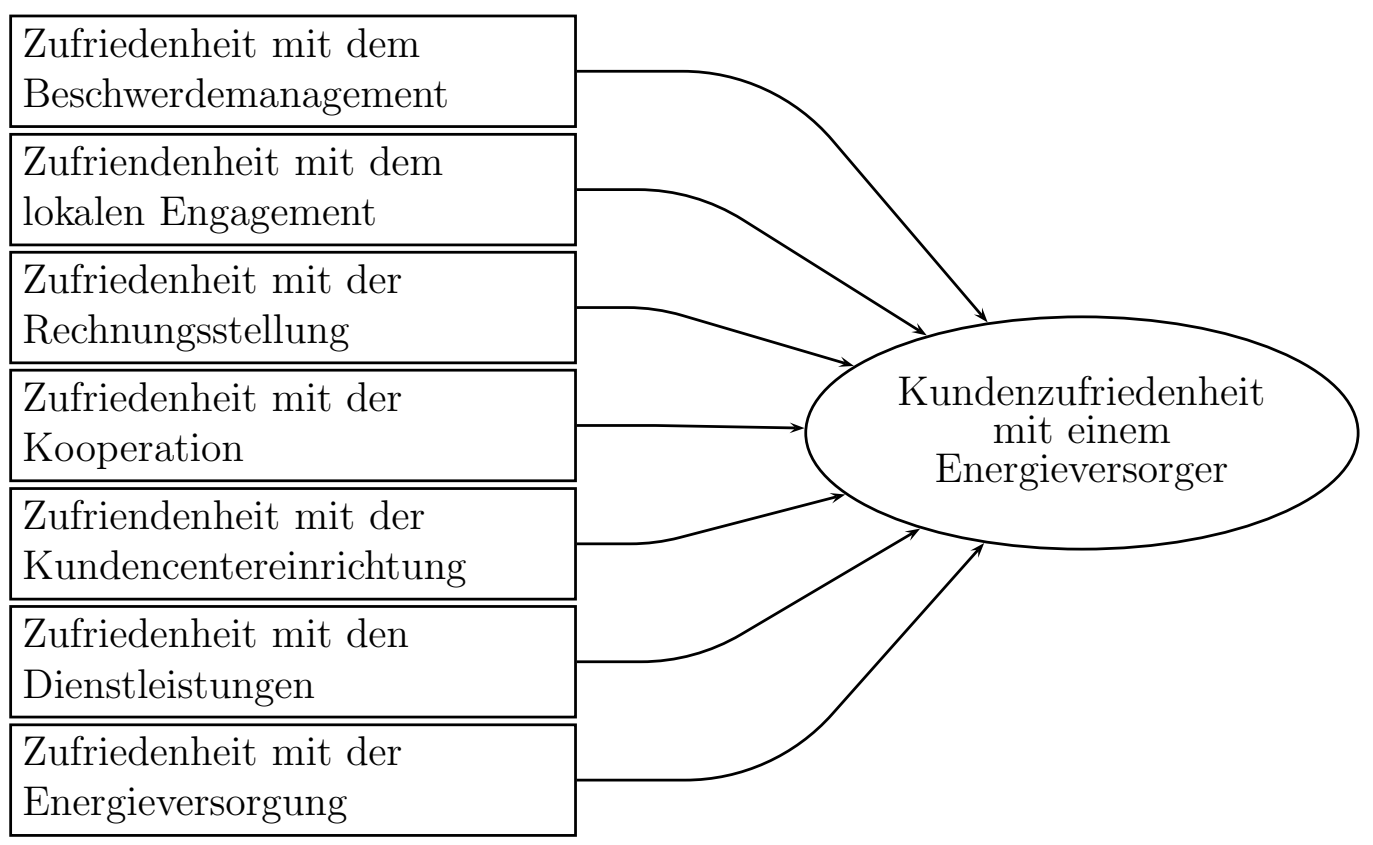

Abbildung 4.1: Teilaspekte der Kundenzufriedenheit mit einem Energieversorger. Angelehnt an BEutin und Werner (1999, S. 554) 
kundenbezogenen Qualitätsbegriff verbirgt sich nichts anderes als „, [.. ] die Kundenwahrnehmung der Produkteigenschaften bzw. Leistungen vom Kunden“ (BRUHN, 2000, S. 25). Daher wird die Kundenzufriedenheit oftmals mit der Dienstleistungsqualität gleichgesetzt (vgl. z. B. Stauss und Hentschel, 1992, S. 115).

Während zahlreiche Dienstleistungsforscher vehement bestreiten, dass es sich bei Kundenzufriedenheit und Dienstleistungsqualität um dasselbe Konstrukt handelt, hat sich die Praxis längst über den akademischen Disput hinweggesetzt: Boulding ET AL. (1993) stellen fest, dass in der Praxis und in der Populärliteratur zwischen Kundenzufriedenheit und Dienstleistungsqualität quasi nicht unterschieden wird. Eine Unterscheidung zwischen beiden Konstrukten ist jedoch möglich und in vielen Situationen auch zweckmäßig. Für eine Unterscheidung zwischen Kundenzufriedenheit und Dienstleistungsqualität lässt sich eine Reihe von Vergleichsdimensionen heranziehen (s. Tab. 4.1 für eine Zusammenfassung der Unterschiede zwischen Dienstleistungsqualität und Kundenzufriedenheit). Die Dimensionen von Qualitätsurteilen sind sehr speziell auf das spezifische Produkt bezogen, wie bspw. Attribute (Steenkamp, 1990, S. 314). Im Gegensatz dazu können Zufriedenheitsurteile auf den unterschiedlichsten Dimensionen beruhen, seien sie qualitätsbezogen oder nicht (vgl. z. B. Garland und Westbrook, 1989; Singh, 1991). Die Erwartungen an die Qualität basieren auf Idealen oder Vorstellungen von „Exzellenz“, wohingegen eine Reihe nicht-qualitativer Referenzen, wie bspw. Bedürfnisse (WEstBrook und REILly, 1983) oder Gerechtigkeitsauffassungen (Oliver und SwAN, 1989), in Kundenzufriedenheitsurteile einfließen. Während Qualitätswahrnehmungen keiner Erfahrung mit der Dienstleistung oder dem Anbieter bedürfen, fußt Kundenzufriedenheit rein auf Erfahrungen (OLIVER, 1993, S. 76). Dienstleistungsqualität hat eine eher geringere Zahl von

Vergleichsdimension

Attribute oder Dimensionen

Referenz der Erwartung

Abhängigkeit von

Erfahrungen

Andere konzeptionelle

Antezedenzen
Dienstleistungsqualität

bezogen auf Qualitätsurteile

Ideale, „Exzellenz“

Erfahrungen nicht erforderlich, können extern mediiert werden

Kommunikation

\section{Kundenzufriedenheit}

alle denkbaren Dimensionen möglich

Voraussagen, Normen, Bedürfnisse etc.

Erfahrungen notwendig

Attribution, Emotionen, Gerechtigkeit etc.

Tabelle 4.1: Vergleich der Konzepte Dienstleistungsqualität und Kundenzufriedenheit. Leicht modifiziert übernommen aus OLIVER (1993, S. 76) 
konzeptionellen Antezedenzen; lediglich persönliche und unpersönliche Kommunikation spielen eine größere Rolle (ZeithamL ET AL., 1991). Hingegen wird Kundenzufriedenheit bekanntermaßen durch zahlreiche kognitive und affektive Prozesse, u. a. Gerechtigkeit, Attribution und Emotion, beeinflusst.

Unterscheidet man zwischen Kundenzufriedenheit und Dienstleistungsqualität, so stellt sich die Frage nach dem Wirkzusammenhang und insbesondere der Wirkrichtung zwischen beiden Konstrukten. Ältere Quellen (vgl. z. B. PArasuraman ET AL., 1985, 1988; Bolton und Drew, 1991) vertreten die Auffassung, dass Kundenzufriedenheit zu wahrgenommener Dienstleistungsqualität führt. In der neueren Forschung (vgl. z. B. Oliver, 1993; Parasuraman Et Al., 1994; Rust und Oliver, 1994; Strandvik und LiluanDER, 1994; DE RUYTER ET AL., 1997) setzt sich jedoch mehr und mehr die Erkenntnis durch, dass die wahrgenommene Dienstleistungsqualität der Kundenzufriedenheit vorgeschaltet ist.

TEAS (1993) schlägt einen konzeptionellen Rahmen vor, der beide Wirkrichtungen zwischen Dienstleistungsqualität und Kundenzufriedenheit integriert: Hierzu unterscheidet er zwischen der transaktionsspezifischen wahrgenommenen Dienstleistungsqualität, die eine Leistungskomponente der transaktionsbezogenen Kundenzufriedenheit darstellt, und der Beziehungsqualität, die in ihrer langfristigen Ausprägung wesentlich durch die kumulierte transaktionsbezogene Kundenzufriedenheit beeinflusst wird. Abb. 4.2 illustriert, wie sich die Gesamtzufriedenheit aus der transaktionsbezogenen Zufriedenheit zusammensetzt. Die in gestrichelten Rahmen gezeichneten Teilmodelle in Abb. 4.2 verdeutlichen den konzeptionellen Ansatz, dass sich die transaktionsbezogene Zufriedenheit $\left(Z_{n}\right)$ aus den Evaluationen von Dienstleistungsqualität, Produktqualität (als Eigenschaft materieller Produkte) und Preis bei der jeweiligen Transaktion ergibt.

In den folgenden Ausführungen wird Kundenzufriedenheit in Anlehnung an BiTnER und Hubbert (1994) sowie Anderson und Fornell (1994) als die Gesamtbewertung erbrachter Leistung, basierend auf allen vorangegangenen Erfahrungen mit einem Unternehmen, aufgefasst. Die hier betrachtete Kundenzufriedenheit ist im Sinne von OLIVER (1997, S. 13) ein subjektives Urteil darüber, ob ein als angenehm empfundener Zustand in einer Konsumerfahrung erreicht wurde. Vereinfacht handelt es sich um die Kundenzufriedenheit mit einem Unternehmen.

Wie wirkt Kundenzufriedenheit auf die Wechselabsicht bzw. auf den Wechselvollzug von Konsumenten? Hierzu stellt Unterabschnitt 4.1.2 Hypothesen auf. 

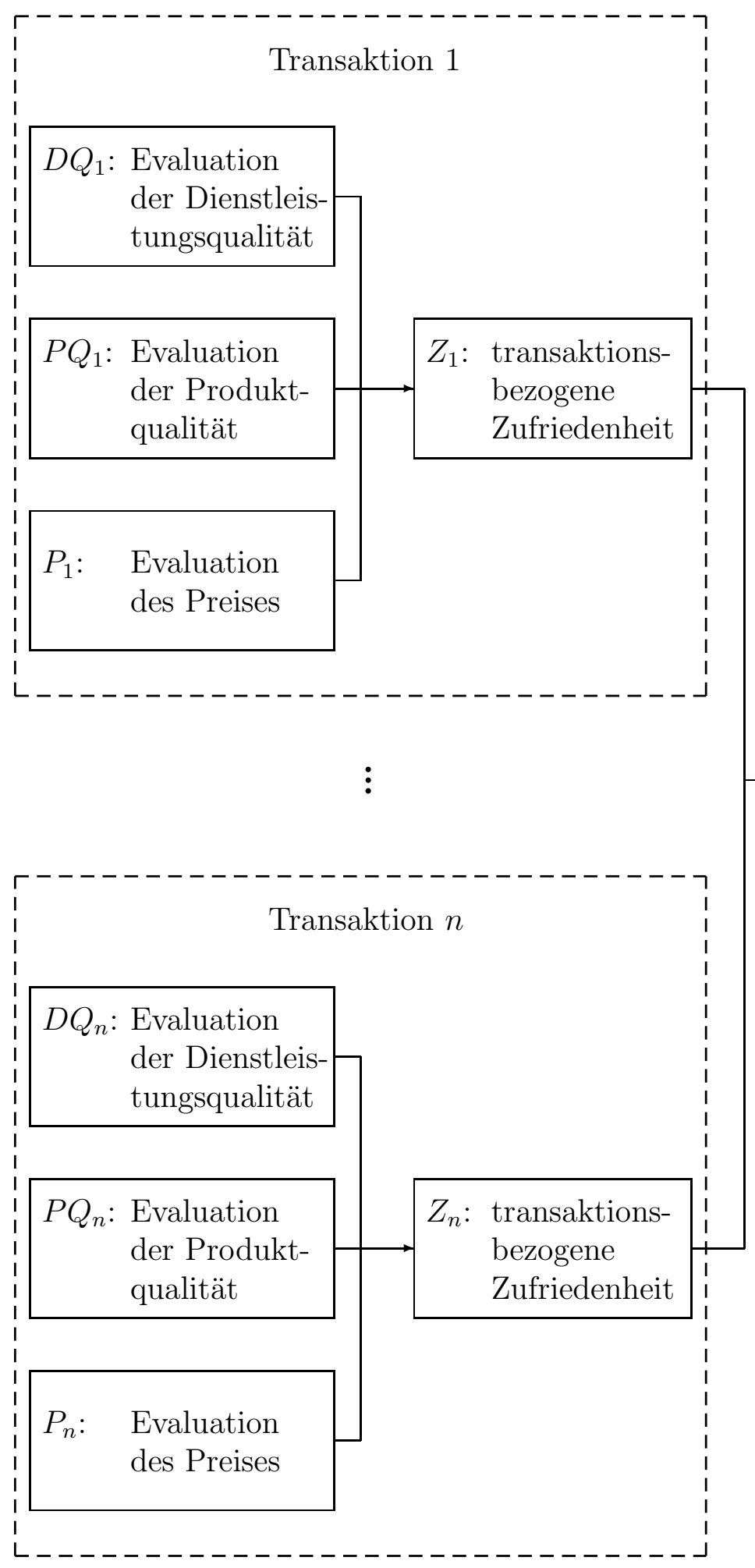

Gesamteindruck vom Unternehmen

- Zufriedenheit

- Beziehungsqualität

- Produktqualität

- Preis

Abbildung 4.2: Komponenten globaler Evaluierungen. In Anlehnung an PARASURAMAN ET AL. (1994, S. 121f.) 


\subsubsection{Hypothesen zum Einfluss der Kundenzufriedenheit bei Versorgungsbeziehungen}

Schon in früherer Forschung wurde die Kundenzufriedenheit als Antezedenz der Wechselabsicht vorgeschlagen (vgl. bspw. Oliver, 1981; BeArden und TEeL, 1983). Zwar unterzogen weder Oliver noch BeARDEN und TeEL den Zusammenhang zwischen Kundenzufriedenheit und Wechselabsicht einer empirischen Überprüfung, doch arbeiteten sie die Kausalbeziehung zwischen den beiden Konstrukten heraus. In einem Produktwahlkontext zeigten später OLIVER und Swan (1989), dass Kundenzufriedenheit in starker Verbindung zur Wiederkaufabsicht steht. Zahlreiche Studien stützen den aufgezeigten Zusammenhang zwischen Kundenzufriedenheit und Wechselverhalten, darunter die von Andreasen (1985), Fornell (1992), Fornell und Wernerfelt (1987) sowie Oliva ET AL. (1992). In der Dienstleistungsforschung ließ sich empirisch zeigen, dass Kundenzufriedenheitsurteile unmittelbare Ursachen von Wechselabsichten sind (vgl. z. B. CrONin und TAYlor, 1992; Gotlieb Et AL., 1994; Rust und ZAhorik, 1993; Woodside ET AL., 1989). Die Ergebnisse dieser Studien legen nahe, dass aufgrund von Unzufriedenheit die Wahrscheinlichkeit zunimmt, dass ein Kunde seinen Anbieter wechselt. Zahlreiche empirische Studien konnten belegen, dass die Kundenzufriedenheit einen positiven Einfluss auf die Kundenloyalität hat, darunter bspw. Bloemer et AL. (1999), Oliver (1999) und Zeithaml et AL. (1996).

Teilweise wird Kundenzufriedenheit nicht isoliert konzeptualisiert, sondern in das breitere Konstrukt Beziehungsqualität eingepasst. Auch die Beziehungsqualität - zusammengesetzt aus der Kundenzufriedenheit und dem Kundenvertrauen - erhöht die Absicht von Konsumenten, bei ihrem Anbieter zu verbleiben (Crosby ET AL., 1990, S. 75). Der Einfluss der Beziehungsqualität auf die Kundenbindung ist auch Thema der Untersuchungen von De Wulf et al. (2001) und Hennig-Thurau et al. (2002). De Wulf et al. (2001, S. 43) zeigen, dass Investitionen eines Anbieters in die Kundenbeziehungen zu einer verbesserten Beziehungsqualität führen und eine bessere Beziehungsqualität ein loyaleres Verhalten der Konsumenten impliziert.

Die Absicht von Konsumenten, bei ihrem Anbieter zu verbleiben, lässt sich insbesondere durch die dargebotene Qualität steigern (Boulding ET AL., 1993). Rust und ZAHORIK (1993) demonstrieren dies an einem Beispiel aus der Bankindustrie, Bitner (1990) bezieht sich auf Dienstleistungsschalter in der Tourismusbranche. Eine branchenübergreifende Studie von Cronin und TAYlor (1992) kommt allerdings zu dem Ergebnis, dass Dienstleistungsqualität alleine keinen signifikanten Einfluss auf die Verhaltensabsicht hat. Jedoch hat sie signifikante Auswirkungen auf die Kundenzufriedenheit, die wiederum die Verhaltensabsicht beeinflusst. Abb. 4.3 verdeutlicht, dass die kumulative Kundenzufrie- 
denheit als ein vollständiger Mediator der Kundenbindungswirkung von Dienstleistungsqualität angesehen werden kann.

Nicht nur in der Marketingwissenschaft, sondern auch in der Praktikerliteratur wird die Wirkung von Kundenzufriedenheit auf das Wechselverhalten diskutiert. So finden sich in der Praktikerliteratur zahlreiche Plädoyers für Kundenzufriedenheit als Schaffer von Kundenbindung (vgl. stellvertretend für viele Brachat, 1995; Müller und RIESEnBECK, 1991; TöPFER und WIEDER, 1996).

Ausgehend sowohl von vorhandenen Theorien - in diesem Fall der Theorie der kognitiven Dissonanz, der Lerntheorie der instrumentellen Konditionierung und der Austauschtheorie - als auch von der großen empirischen Evidenz der Bindungswirkung von Kundenzufriedenheit liegen die folgenden beiden Hypothesen nahe:

\section{Hypothese 1}

Die Gesamtzufriedenheit eines Kunden mit seinem Anbieter steht in gegensätzlichem Verhältnis zur Intention, den Anbieter zu wechseln.

\section{Hypothese 2}

Die Gesamtzufriedenheit eines Kunden mit seinem ursprünglichen Anbieter steht in gegensätzlichem Verhältnis zum Wechselvollzug.

Kundenzufriedenheit besitzt eine herausragende Bedeutung für eine Bindung des Kunden an das Unternehmen (PETER, 1999, S. 105). Zwar kann Kundenzufriedenheit als Grundvoraussetzung für eine dauerhafte Bindung angesehen werden, jedoch nicht als Garantie. Empirische Studien belegen, dass es trotz hoher Zufriedenheit mit dem Angebot eines Unternehmens immer wieder zu vermehrter Abwanderung der Kunden kommt (StAuss

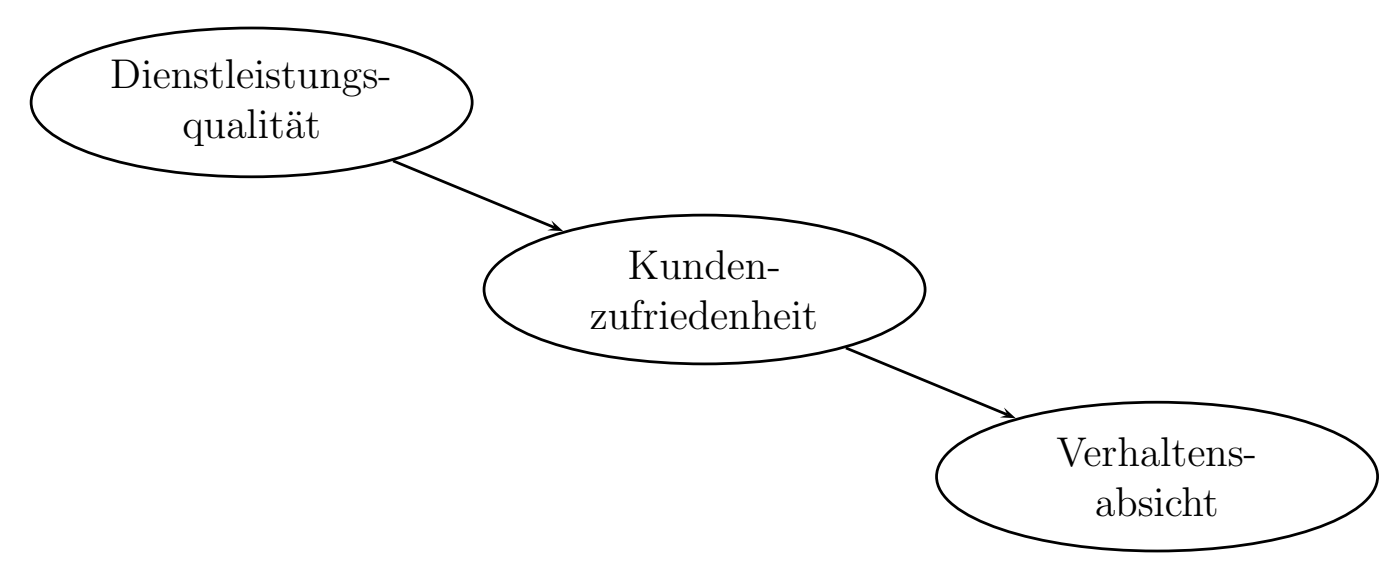

Abbildung 4.3: Wirkzusammenhang zwischen Dienstleistungsqualität, Kundenzufriedenheit und Verhaltensabsicht 
und Neuhaus, 1995, S. 4f.). „Die Erreichung von Kundenzufriedenheit ist vielmehr notwendige Voraussetzung, um eine emotionale Bindung der Kunden aufzubauen" (EGGERT und HeLm, 2000, S. 63).

Zweifel an der Allgemeingültigkeit des Zusammenhangs zwischen Kundenzufriedenheit und Kundenloyalität mehren sich jedoch (Homburg ET AL., 1999, S. 181). In der Literatur herrscht Einvernehmen darüber, dass auch bei zufriedenen Kunden Wechselbereitschaft vorhanden sein kann (vgl. beispielhaft für viele: MAZURSKY ET AL., 1987, S. 20-22; Weinberg, 1977, S. 43ff.). GierL (1993) berichtet, dass viele Unternehmen die Erfahrung gemacht haben, dass selbst zufriedene Kunden abwandern bzw. die Marke wechseln. Er macht daher in diesem Kontext darauf aufmerksam, dass „,selbst Zufriedenheit mit dem Produkt [..] nicht vor Untreue [schützt], denn gerade zufriedene Kunden gehören zu dauerhaften Markenwechslern" (GIERL, 1993, S. 90). Um mit den Worten von STURM und THIRY (1991, S. 34) zu sprechen: "A satisfied customer is a repeat customer-maybe." ReichHeld und Aspinall (1994, S. 25f.) räumen daher ein, dass Kundenzufriedenheit „[... n not a particularly reliable indicator of customer's future action" ist.

Zwar konnten zahlreiche empirische Studien belegen, dass Kundenzufriedenheit positiv die Fortsetzung von Beziehungen beeinflusst (vgl. z. B. Fornell, 1992; Oliver und SwAN, 1989 und TAYLOR und BAKER, 1994) bzw. dass sie sich negativ auf die Wechselabsicht von Kunden auswirkt (vgl. beispielhaft Mittal et Al., 1998 und Hennig-Thurau et Al., 2002). Allerdings ist der Einfluss der Kundenzufriedenheit keineswegs immer signifikant. Anderson und Sullivan (1993) finden im Rahmen einer Meta-Untersuchung für die Hypothese „die Kundenzufriedenheit wirkt positiv auf die Fortsetzung einer Beziehung“ Variationen des Student-t-Wertes zwischen 1,1 und 13,1 vor. Die Hypothese kann also nicht in allen Fällen empirisch gestützt werden. Diese großen Variationen der Signifikanz legen nahe, dass noch weitere Faktoren außer der Kundenzufriedenheit einen maßgeblichen Einfluss auf den Verbleib in einer Beziehung haben.

\subsection{Attraktivität der Alternativen als Determinante des Wechselverhaltens}

Geschäftsbeziehungen im Allgemeinen und Versorgungsbeziehungen im Besonderen bestehen aus einer Folge von Transaktionen. Unter Transaktionen ist zu verstehen, dass Anbieter und Nachfrager Dinge von Wert austauschen. Ein solcher Austausch kommt nur dann zu Stande, wenn beide Marktparteien sich durch ihn gegenüber der Ausgangssituation besser oder zumindest nicht schlechter stellen (KotLer und BliEmel, 2001, S. 5). Auch das Zustandekommen eines Anbieterwechsels lässt sich damit erklären. Dabei liegt 
die Besonderheit beim Anbieterwechsel in der Ausgangssituation: Der Nachfrager tauscht bereits konkrete Dinge von Wert mit einem bestimmten Anbieter aus, somit dient die aktuelle Geschäftsbeziehung dem Nachfrager als Vergleichsmaßstab für die Beurteilung des Nutzens potenzieller Alternativen (WEIBER und AdLER, 2003, S. 79). Entscheidend für einen Anbieterwechsel ist dann, dass sich der Nachfrager durch einen Austausch mit einem anderen als dem gegenwärtigen Anbieter besser oder zumindest nicht schlechter stellt. Existieren keine attraktiven Alternativen, so befinden sich Kunden in einer Abhängigkeit von ihrem bisherigen Anbieter (EMERson, 1976). Die Attraktivität der Alternativen ist eine notwendige Bedingung für das Zustandekommen eines Anbieterwechsels.

Zunächst nimmt Unterabschnitt 4.2.1 eine Konzeptualisierung der Attraktivität der Alternativen vor. Dann bildet Unterabschnitt 4.2.2 Hypothesen zu direkten Wirkbeziehungen der Attraktivität der Alternativen im Rahmen des Wechselverhaltens von Konsumenten bei Versorgungsbeziehungen. Abschließend stellt Unterabschnitt 4.2.3 Hypothesen zum moderierenden Einfluss der Attraktivität der Alternativen auf.

\subsubsection{Konzeptualisierung der Attraktivität der Alternativen}

Bei der Konzeptualisierung der Attraktivität der Alternativen sind drei Fragen zu beantworten: worauf sich Attraktivität bezieht, ob die Attraktivität der Alternativen zu den Wechselkosten gezählt wird und ob die Wechselkosten mit der Attraktivität der Alternativen verrechnet werden sollen.

Wann genau liegen attraktive Alternativen vor? Wenn es mindestens eine attraktive Alternative gibt, wenn es deren viele gibt, wenn sie im Durchschnitt attraktiv sind, oder wenn die beste verfügbare Alternative attraktiv ist? Hierüber herrscht in der Literatur keine einheitliche Auffassung. Für Ping (1993) sowie Patterson und Smith (2003) ist es entscheidend, dass mindestens eine attraktive Alternative verfügbar ist: „Alternative attractiveness is conceptualized as the client's estimate of the likely satisfaction available from an alternative relationship" (PATterson und Smith, 2003, S. 109). Jones ET AL. (2000) sowie JEN und Hu (2003) subsummieren unter der Attraktivität der Alternativen die Wahrnehmungen von Kunden über das Ausmaß, in dem brauchbare Wettbewerber am Markt verfügbar sind: „[..] the attractiveness of competing alternatives [..] i. e., the number of acceptable firms from which to choose [... " (Jones ET AL., 2000, S. 263). Für ThAu ET AL. (2004) kommt es darauf an, dass die Alternativen relativ attraktiv sind. In der Tradition der Austauschtheorie stimmt die Attraktivität der Alternativen mit der Belohnung überein, die sich aus dem Wechsel der Anbieter erhalten lässt. Entscheidend ist hierbei die attraktivste Alternative. So konstatiert Homans (1974, S. 31): „We have in mind the best alternative, the one that would have brought the most valuable reward." 
Unter der Attraktivität der Alternativen ist dann die Attraktivität derjenigen Alternative zu verstehen, durch die sich der maximale Wechselgewinn erzielen lässt.

Handelt es sich bei der Attraktivität der Alternativen um eine Form von Wechselkosten? Schließlich nennen zahlreiche Autoren die Attraktivität der Alternativen in einem Atemzug mit Wechselkosten oder bezeichnen sie explizit als eine Wechselbarriere (vgl. z.B. Jones et AL., 2000, S. 261; Kim et AL., 2004, S. 149). Colgate und Lang (2001, S. 338f.) zeigen anhand empirischer Studien, in denen sie Versicherungs- und Bankkunden befragten, dass die Attraktivität der Alternativen und Wechselkosten auf einen Faktor laden, d. h. statistisch stark miteinander verwandt sind. Trotzdem ist die Zusammenfassung der Wechselkosten und der Attraktivität der Alternativen inhaltlich wenig sinnvoll: Während die Wechselkosten, wie der Name bereits aussagt, die Kosten eines Anbieterwechsels umfassen, handelt es sich bei der Attraktivität der Alternativen um den Nutzen, der sich aus einem Anbieterwechsel ziehen lässt. Eine Zusammenfassung beider Größen, also eine Saldierung, geht mit einem Informationsverlust einher und widerspricht betriebswirtschaftlichen Prinzipien. Mit Wechselkosten und ihrer Rolle im Rahmen des Wechselverhaltens von Konsumenten beschäftigt sich daher separat der Abschnitt 4.3.

In Bezug auf die Attraktivität der Alternativen lassen sich zwei Perpektiven einnehmen: eine Bruttoperspektive, aus der die Attraktivität der Alternativen als der Nutzenzugewinn durch einen Anbieterwechsel anzusehen ist, sowie eine Nettoperspektive, bei der der Nutzenzugewinn mit den Wechselkosten saldiert wird. WeIBER und AdLER (2003) diskutieren die Attraktivität der Alternativen im Sinne einer Nettonutzendifferenz. Die Konzeptualisierung der Attraktivität der Alternativen als Nettonutzendifferenz erweist sich jedoch als nachteilig, wenn in einem Modell zusätzlich die wahrgenommenen Wechselkosten betrachtet werden. Schließlich hängen diese beiden Größen unmittelbar voneinander ab:

$$
\text { Nettonutzendifferenz }=\text { Bruttonutzendifferenz }- \text { Wechselkosten }
$$

Die Varianz der Nettonutzendifferenz würde zu einem großen Teil mit der Varianz der Wechselkosten übereinstimmen. Zwei negative Konsequenzen können sich dadurch ergeben: Erstens lässt sich der Einfluss der Wechselkosten auf das Wechselverhalten nicht mehr einzeln quantifizieren. Wie vom Problem der Multikollinearität allgemein bekannt, lassen sich dann die Modellparameter nicht mehr sinnvoll interpretieren. Zweitens kann die hohe Korrelation zwischen den Wechselkosten und der Attraktivität der Alternativen einer diskriminanzvaliden Messung beider Konstrukte entgegenstehen.

Aufgrund der genannten Nachteile der Nettoperspektive wird im Folgenden die Bruttoperspektive eingenommen, d.h. für die vorliegende Arbeit wird unter der Attraktivität der Alternativen der Wechselgewinn verstanden, der sich durch die Wahl der vorteilhaftesten 
Alternative erzielen lässt. Da angesichts der Homogeneität des Produkts Strom Qualitätsunterschiede als Differenzierungsmerkmal zwischen Anbietern nicht in Frage kommen, spiegelt sich die Attraktivität der Alternativen allein in der möglichen Ersparnis durch einen Anbieterwechsel wider.

\subsubsection{Hypothesen zum Einfluss der Attraktivität der Alternativen bei Versorgungsbeziehungen}

Ein neuer potenzieller Anbieter mit günstigeren Preisen ermöglicht einem Käufer sofortige Kostenersparnisse (WATHne ET AL., 2001, S. 55). Über eine bestimmte Zeitspanne hinweg können diese Ersparnisse beträchtlich werden (KRANTON, 1996). Daher erschließt sich intuitiv folgender Zusammenhang: Je niedriger die Preise neuer Anbieter relativ zum bisherigen Anbieter sind, desto größer ist der ökonomische Nutzen eines Anbieterwechsels und desto größer ist die Wahrscheinlichkeit, dass ein Kunde seinen Anbieter wechselt. WAthne ET AL. (2001, S. 55) weisen darauf hin, dass dieser intuitive Ansatz die Erkenntnisse existierender Theorien zu Preisreaktionen von Konsumenten außer Betracht lässt. $\mathrm{Zu}$ unterscheiden ist zwischen zwei Forschungslinien: der Informationsverarbeitung und der Informationsökonomie. Forschung in der Tradition der Informationsverarbeitung legt nahe, dass Kunden einen geringeren Preis mit geringerer Qualität in Verbindung bringen können (MonRoE und DodDS, 1988). Ein niedriger Preis von Konkurrenzanbietern würde insofern nicht zwingend einen Anreiz zum Wechseln liefern. Eine gegensätzliche Vorhersage bietet die Informationsökonomie an: Ein niedriger Einführungspreis dient hier als Signal für eine hohe Qualität. Schmalensee (1978) erklärt dies so: Ein Anbieter hochqualitativer Produkte kann versuchen, durch einen niedrigen Preis Konsumenten da$\mathrm{zu} \mathrm{zu}$ veranlassen, seine Produkte zu probieren. In der nicht unberechtigten Erwartung auf mehr Wiederholungskäufe opfert das Unternehmen Profit in der Gegenwart, um in der Zukunft einen höheren Gewinn zu erzielen. Ein Anbieter von Produkten niedriger Qualität kann nicht auf Wiederkäufe vertrauen, sodass es sich für ihn nicht lohnt, ein derartiges Signal auszusenden. Die beiden Forschungslinien unterscheiden sich auch in ihrem Verständnis vom Konsumenten (KIRMAnI und RAO, 2000): In der Tradition der Informationsverarbeitung steht der "faule" Konsument, der Preisinformation zur kognitiven Vereinfachung einsetzt. Hingegen verfolgt die Informationsökonomie das Bild eines rational handelnden Konsumenten, der auch die impliziten Bindungen bewertet, die einzelnen Signalen zu Grunde liegen. Da es sich bei Strom um ein extrem homogenes Produkt handelt, bietet die Produktqualität keine Differenzierungsmöglichkeit. Selbst wenn sich also die durch die beiden Forschungslinien beschriebenen Effekte nicht egalisieren, dürfte ihr zusätzlicher Erklärungsbeitrag gering sein. 
Aus der Perspektive der Austauschtheorie ist die Attraktivität der Alternativen neben der Kundenzufriedenheit der zweite große Bestimmungsfaktor für die Fortsetzung von Beziehungen (KNOESTER und BoоTh, 2000). Sie bestimmt maßgeblich die Lage des individuellen Vergleichsstandards für Alternativen, $C L_{\text {alt }}$. Der Vergleich der Differenz aus Belohnungen und Kosten der aktuellen Beziehung mit dem Vergleichsstandard der Alternativen entscheidet darüber, ob eine Person eine Beziehung fortsetzt oder beendet. Wenn Konsumenten nur wenig attraktive Alternativen zur Auswahl stehen, sollte der durch einen Wechsel erzielbare Nutzen gering sein. Dies dürfte dazu führen, dass Konsumenten ihren Anbieter eher nicht wechseln.

Empirische Unterstützung für ihren Einfluss auf die Fortsetzung von Beziehungen liefern z. B. Rusbult (1980) im Kontext zwischenmenschlicher Beziehungen, Hom und Kinicki (2001) sowie FARrell und Rusbult (1981) im Zusammenhang mit Mitarbeiterfluktuation. JEN und HU (2003, S. 319) entdecken einen signifikanten Einfluss der Attraktivität der Alternativen bei der (Wieder-)Wahl von Transportmitteln. Ping (1993) zeigt den Einfluss der Attraktivität der Alternativen auf die Stabilität von Beziehungen in Distributionskanälen. Ist die Attraktivität der Alternativen gering, oder gibt es kaum alternative Wettbewerber, bleiben Konsumenten tendenziell ihrem Anbieter treu (BENDAPUDI und Berry, 1997). ANDERson und NARus (1990) führen den Umstand, dass Konsumenten von ihrem Anbieter abhängig sind, auf den Mangel an stärkeren Wettbewerbern im Markt zurück. Unter diesen Gegebenheiten bleiben Kunden selbst dann bei ihrem Anbieter, wenn sie mit ihm nicht zufrieden sind, da sie sich mit einem anderen Anbieter nicht besser stellen würden.

Für die Wirkweise der Attraktivität der Alternativen im Rahmen des Wechselverhaltens von Konsumenten lässt sich die folgende Hypothese aufstellen:

\section{Hypothese 3}

Je attraktiver für einen Konsumenten die alternativen Anbieter sind, desto eher hat er die Intention, seinen Anbieter zu wechseln.

In Anlehnung an GierL (1993, S. 90) lassen sich die Konsumenten, die den Anbieter wechseln, in zwei Kategorien einteilen: Die Konsumenten der ersten Kategorie wechseln, weil sie mit ihrem alten Anbieter unzufrieden sind und durch Marketingmaßnahmen oder per Weiterempfehlung einen anderen Anbieter kennen lernen, der sie dann eher zufrieden stellt. Die Konsumenten der zweiten Kategorie wechseln ihren Anbieter, obwohl sie bislang mit ihm zufrieden waren. Im letzteren Fall müssen auf die Konsumenten starke Stimuli einwirken, die vorwiegend preislicher Natur sind (GIERL, 1993, S. 91). Bezogen auf den Wechselvollzug lässt sich somit die folgende Hypothese aufstellen: 


\section{Hypothese 4}

Eine höhere Attraktivität der Alternativen geht einher mit eher realisiertem Wechselvoll$z u g$.

Obwohl die beiden Hypothesen unmittelbar einsichtig, ja nahezu trivial anmuten, und sich - nimmt man die Perspektive des „homo oeconomicus“ ein - kaum gegen sie argumentieren lässt, finden sich doch empirische Studien, die die Hypothese nicht bestätigen können: Sharma und Patterson (2000, S. 490) finden bspw. keinen direkten Zusammenhang zwischen der Attraktivität der Alternativen und dem Commitment von Kunden in einer Geschäftsbeziehung, wobei Sharma und Patterson (2000, S. 489) Commitment u. a. auch als Absicht operationalisieren, die Geschäftsbeziehung beizubehalten. Auch KIM ET AL. (2004), die u. a. den Einfluss der Attraktivität der Alternativen auf die Kundenloyalität im koreanischen Mobilfunkmarkt prüften, konnten keinen signifikanten direkten Effekt feststellen. Allerdings wecken drei Indizien Zweifel an ihren Ergebnissen: erstens die reflektive Messung der Attraktivität der Alternativen mittels der Indikatoren Ruf, Image und Dienstleistungsqualität des Ersatzanbieters; zweitens die Vernachlässigung des Preisgefüges und drittens die schlechte Modellanpassung.

Nachdem nun die direkten Effekte der Attraktivität der Alternativen herausgearbeitet worden sind, geht der folgende Unterabschnitt auf mögliche Interaktionseffekte der Attraktivität der Alternativen ein.

\subsubsection{Attraktivität der Alternativen als Moderator der Wirkung der Kundenzufriedenheit}

Mittels qualitativer Interviews gelangten Sharma und Patterson (2000) zu der Erkenntnis, dass die Verfügbarkeit konkurrierender Anbieter Kunden dazu motivieren kann, die Beziehung mit ihrem Anbieter aufzulösen. Insbesondere die folgende Kundenaussage verdeutlicht dies: "I think another adviser will charge less fees and give better performance. They might take care of my queries and return important calls" (SHARma und Patterson, 2000, S. 477). Sharma und Patterson schließen daraus, dass ein Kunde, der lediglich mäßig (nicht seinen Erwartungen entprechend) mit seinem Anbieter zufrieden ist, einen Anbieterwechsel eher in Betracht zieht, wenn attraktive Alternativen verfügbar sind. Die Bereitschaft, eine nicht zufriedenstellende Geschäftsbeziehung beizubehalten, verringert sich durch das Vorhandensein attraktiver Alternativen.

Die obigen Betrachtungen zu einem hypothetischen moderierenden Effekt der Attraktivität der Alternativen münden in die folgenden beiden Hypothesen. Die Hypothese 5 
bezieht sich auf den moderierenden Einfluss, den die Attraktivität der Alternativen auf die Beziehung zwischen der Kundenzufriedenheit und der Wechselabsicht ausübt:

\section{Hypothese 5}

Je attraktiver die Alternativen sind, desto eher führt eine geringere Zufriedenheit eines Kunden mit seinem Anbieter zu der Absicht, den Anbieter zu wechseln.

Jones und SASser (1995, S. 91) merken an, dass in Situationen mit großer Konkurrenz unzufriedene Kunden ihren Anbieter schnell wechseln. Ist kaum Konkurrenz vorhanden oder sind die Alternativen den Kunden nicht bewusst, verbleiben auch Kunden mit geringer Kundenzufriedenheit in der Kundenbeziehung. Hier zeigt sich der moderierende Einfluss der Attraktivität der Alternativen auf die Beziehung zwischen der Kundenzufriedenheit und dem Wechselvollzug.

Die Hypothese 6 bezieht sich auf diesen moderierenden Einfluss der Attraktivität der Alternativen auf die Beziehung zwischen der Kundenzufriedenheit und dem Wechselvollzug:

\section{Hypothese 6}

Je attraktiver die Alternativen sind, desto eher führt eine geringere Zufriedenheit eines Kunden mit seinem Anbieter dazu, dass der Kunde den Anbieter wechselt.

\subsection{Wechselkosten als Determinante des Wechselverhaltens}

Konsumenten verbleiben nicht nur in Versorgungsbeziehungen, weil sie es wollen. Selbst wenn attraktive alternative Angebote verfügbar sind, muss ein Anbieterwechsel für einen Konsumenten nicht unbedingt nutzenstiftend sein: Wechselbarrieren bzw. die durch diese induzierten Wechselkosten können den Anbieterwechsel für Konsumenten unattraktiv machen, bspw. indem sie gemäß der Austauschtheorie den Vergleichsstandard für Alternativen $C L_{\text {alt }}$ herabsetzen. In ihrer extremsten Form können Wechselbarrieren einen Anbieterwechsel sogar gänzlich vereiteln.

Wechselkosten erhalten zunehmend Beachtung im Konsumgütermarketing. Beispiele sind u. a. die Arbeiten von Anderson (1994), Maute und Forrester (1993) und Jones ET AL. (2000), die den Einfluss von Wechselkosten auf Konsumgütermärkten untersuchten. Das Phänomen „Wechselkosten“ ist jedoch nicht nur Gegenstand der Konsumentenverhaltensforschung, sondern findet auch in anderen Feldern der Marketingwissenschaft oder der Wirtschaftswissenschaften allgemein Berücksichtigung. So liefern z. B. die Arbeiten von JACKSON (1985) und SöLLnER (1993) Erkenntnisse zum Einfluss von Wechselkosten im Business-to-Business-Marketing. WeIss und Anderson (1992) betrachten die von Managern wahrgenommenen Wechselkosten, die eine Änderung des Vertriebskanals 
mit sich bringen würde. Sie kommen dabei zu dem Ergebnis, dass hohe Wechselkosten einen Wechsel verhindern, auch wenn die aktuelle Situation nicht zufriedenstellend ist (vgl. S. 109). Untersuchungen zur Arbeitnehmerfluktuation zeigen, dass Mitarbeiter ihren Job mit geringerer Wahrscheinlichkeit wechseln, wenn die Kosten eines Jobwechsels steigen (FARRELl und Rusbult, 1981). Auch in der Volkswirtschaftslehre sehen Wissenschaftler das Ausmaß und die Bedeutung von Wechselkosten deutlich steigen (vgl. z. B. KLEMPERER, 1995, S. 536). Im Vergleich zu Konstrukten wie der Kundenzufriedenheit oder der Kundenloyalität hat das Konzept der Wechselkosten jedoch geringe Aufmerksamkeit erlangt. Jones ET AL. (2000, S. 260) kritisieren, dass Wechselbarrieren trotz ihres möglicherweise wichtigen Beitrags zum Kundenbindungsprozess bislang nur eine geringe Beachtung im Marketing gefunden haben, von wenigen Ausnahmen wie z. B. den Arbeiten von Anderson (1994) oder MaUte und Forrester (1993) abgesehen. Wechselkosten sind eine häufig erwähnte, aber nur selten untersuchte Einflussgröße auf das Wechselverhalten von Konsumenten (Burnham ET AL., 2003, S. 110). Dass Wechselkosten bei Versorgungsbeziehungen und speziell im Strommarkt für Haushaltsstromkunden eine wichtige Rolle spielen, dokumentieren die Ausführungen von Fusser (2002, o. S.): Er berichtet, dass ein Anbieterwechsel praktisch für die meisten Konsumenten mit einem unbekannten Aufwand verbunden ist und negative Assoziationen - bspw. mit Behördengängen - hervorruft.

Dieser Abschnitt widmet sich dem Konzept der Wechselkosten und seinem möglichen Beitrag zur Erklärung von Wechselverhalten von Konsumenten im Strommarkt. Zunächst sind die Konzepte „Wechselbarrieren“ und „Wechselkosten“ zu erläutern und voneinander abzugrenzen (Unterabschnitt 4.3.1). Im darauf folgenden Unterabschnitt (4.3.2) werden die Wechselkosten kategorisiert und die einzelnen Wechselkostenarten analysiert. Mit der Rolle von Wechselkosten innerhalb des Wechselverhaltens beschäftigen sich die letzten beiden Unterabschnitte: Zuerst stehen die direkten Effekte von Wechselkosten im Vordergrund (Unterabschnitt 4.3.3), anschließend werden moderierende Effekte betrachtet (Unterabschnitt 4.3.4).

\subsubsection{Wechselkosten vs. Wechselbarrieren}

In der Marketingwissenschaft werden sowohl Wechselkosten als auch Wechselbarrieren als potenzielle Antezedenzen von Wechselverhalten und Kundenloyalität diskutiert. Dabei herrscht Uneinigkeit über die Abgrenzung zwischen den beiden Begriffen. Während PETER (1999, S. 115) feststellt, dass die Begriffe „Wechselkosten“ und „Wechselbarrieren“ oftmals synonym verwendet werden, sehen bspw. MAUTE und ForRESTER (1993, S. 227) Wechselkosten und Wechselbarrieren als zwei verschiedene, aber interdependente Konstrukte an. Diese Arbeit folgt der Auffassung von Maute und Forrester. Demnach 
sind unter Wechselbarrieren all jene Mechanismen zu verstehen, die dem Kunden den Anbieterwechsel erschweren oder für ihn kostspielig machen (ähnlich auch JoNES ET AL., 2000, S. 261). Dem Kunden entstehen Wechselkosten, wenn er diese Wechselbarrieren überwinden möchte. Letzteres ist für einen Anbieterwechsel unerlässlich.

Maute und Forrester (1993, S. 227) beschreiben die Wechselwirkung zwischen Wechselkosten und Wechselbarrieren: Wechselbarrieren wirken nicht unmittelbar auf den Anbieterwechsel ein, sondern erhöhen die den Kunden entstehenden Wechselkosten. Die Wechselkosten wiederum vermindern den Nettonutzen, den Kunden möglicherweise aus einem Anbieterwechsel ziehen können, und tragen auf diese Weise zur Vermeidung von Anbieterwechseln bei. Wechselbarrieren sollten also nicht direkt, sondern erst über den Mediator Wechselkosten zu reduziertem Wechselverhalten führen.

Abb. 4.4 verdeutlicht den Zusammenhang von Wechselbarrieren und Wechselkosten und bettet die beiden Konstrukte in einen weiter gefassten Kontext.

Wechselbarrieren können u. a. von etablierten Anbietern errichtet werden. Je nach Branche haben sich unterschiedliche Formen von Wechselbarrieren entwickelt. Zur Illustration mögen die folgenden Beispiele dienen:

- die Aufblähung des Wechselaktes durch umständliche Formulare und zusätzliche Verwaltungsakte,

- Gebühren für den Anbieterwechsel,

- Beziehungsmarketingprogramme und

- die Betonung des Risikos eines Anbieterwechsels.

Der Staat gibt die Regeln des Marktes vor und entscheidet direkt oder indirekt über die Zulässigkeit bestimmter Wechselbarrieren - sei es als Gesetzgeber, Rechtsprecher, Wettbewerbshüter oder Regulator. Insbesondere bei der Gesetzgebung versuchen sowohl etablierte als auch neue Anbieter bzw. ihre jeweiligen Verbände, Einfluss auf die Gestaltung der Marktregeln zu nehmen. Wechselbarrieren führen dazu, dass ein Wechsel für den Kunden aufwendiger wird und sich mitunter nicht mehr lohnt. Dieser Aufwand spiegelt sich wider in den Wechselkosten, die ein Kunde wahrnimmt. Burnham ET AL. (2003, S. 110) definieren Wechselkosten als "the onetime costs that customers associate with the process of switching from one provider to another." In den oben genannten Beispielen für Wechselbarrieren entstehen Kunden Wechselkosten auf folgende Weise:

- dem Aufwand für die Wechselformalitäten, insbesondere dem Ausfüllen und Versenden von Formularen, 


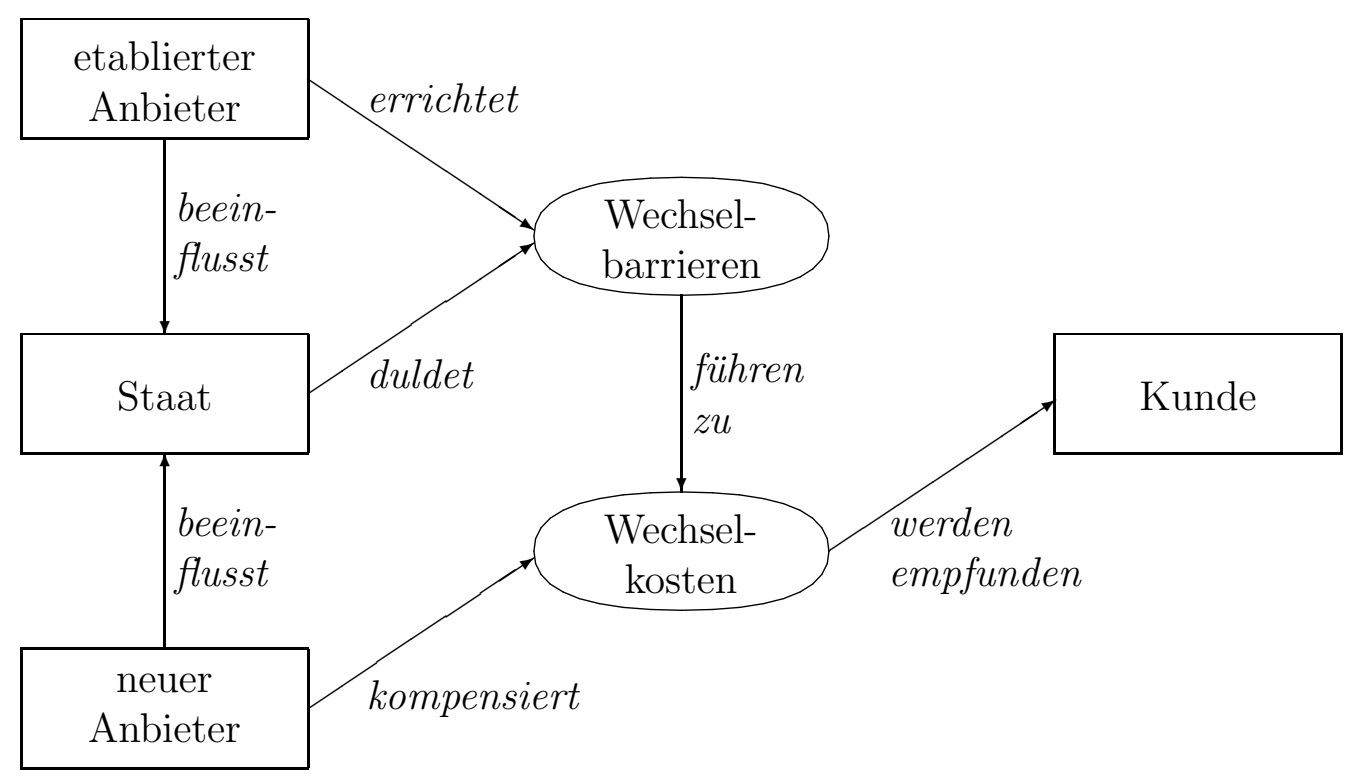

Abbildung 4.4: Wechselbarrieren und Wechselkosten: Vereinfachte Darstellung des weiteren Kontexts

- monetären Kosten für die Beendigung der alten Beziehung,

- dem Verlust der Ansprüche aus Beziehungsmarketingprogrammen und

- der Angst vor negativen Folgen eines Anbieterwechsels.

Neue Anbieter, die Kunden zum Wechseln motivieren wollen, müssen den Kunden helfen, die Wechselbarrieren zu überwinden, d.h. die Wechselkosten möglichst vollständig kompensieren. Sie besitzen verschiedene Möglichkeiten, die vom Kunden wahrgenommenen Wechselkosten zu senken. Sie können, um bei den obigen Beispielen zu bleiben:

- als ein offiziell bestellter Vertreter des Kunden die Wechselformalitäten übernehmen,

- die Kosten erstatten, die der alte Anbieter dem Kunden für den Wechsel in Rechnung stellt,

- dem Kunden in einem eigenen Beziehungsmarketingprogramm zu einem besonderen Status verhelfen und

- vertrauensbildende Maßnahmen durchführen.

Übereinstimmend mit den vorgebrachten Überlegungen argumentieren Wirtschaftswissenschaftler, dass der Einfluss der Wechselkosten auf die Kundenloyalität von zwei rivalisierenden Kräften bestimmt wird (VIARD, 2002): Die eine Kraft bilden die etablierten 
Anbieter, die Wechselbarrieren errichten, um ihre Kunden am Wechseln zu hindern. Die andere Kraft sind die Wettbewerber, die sich entsprechender Strategien bedienen, um die Wirkung der Wechselbarrieren zu neutralisieren. YANG und Peterson (2004, S. 806) nennen als Beispiel die beliebte Praxis von Online-Banken und -Brokern, neuen Kunden eine Geldprämie als Anreiz für einen Anbieterwechsel zuzusichern.

\subsubsection{Arten von Wechselkosten}

Kunden können unterschiedliche Formen von Wechselkosten wahrnehmen. Anders als der Begriff „Wechselkosten“ vielleicht vermuten lässt, sind Wechselkosten nicht allein ökonomischer Natur. Wechselkosten können bspw. auch psychologisch oder emotional sein (Sharma und Patterson, 2000, S. 474; Dick und Basu, 1994, S. 105). Jones et AL. (2002, S. 441) definieren Wechselkosten daher von vornherein als die wahrgenommenen ökonomischen und psychologischen Kosten, die ein Anbieterwechsel mit sich bringt.

Einige Arten von Wechselkosten spielen nur in bestimmten Märkten oder Branchen eine Rolle, während andere Arten allgemein Relevanz besitzen. Um einen Überblick über die unterschiedlichen Arten von Wechselkosten zu erlangen, bietet sich die Betrachtung von Kategorisierungen an. Kategorisierungen von Wechselkosten findet man an vielen Stellen der wirtschaftswissenschaftlichen Literatur. Sie gehen entweder auf konzeptionelle Überlegungen zurück oder entstammen empirischen Analysen.

Eine Reihe von Autoren liefert Aufzählungen von Wechselkostenarten. JACKSON (1985, S. 42-61) identifiziert bspw. zwei Kategorien von Wechselkosten: Die erste Kategorie stellen Investitionen („,investment actions“) in die Geschäftsbeziehungen dar. Hierunter fallen bspw. Anschaffungskosten und speziell für die Geschäftsbeziehung erlernte Prozesse. Die zweite Kategorie ist das Risiko, d.h. mögliche Gefährdungen, die ein Anbieterwechsel mit sich bringt. Eine feinere Unterteilung nimmt ForNelL (1992) vor, der unter Wechselkosten Suchkosten, Transaktionskosten, Lernkosten, Kundentreuerabatte, Kundengewohnheiten, emotionale Kosten und kognitive Anstrengungen zusammen mit finanziellen, sozialen und psychologischen Risiken auf Seiten der Kunden subsummiert. Nach dem Verständnis von KLEMPERER (1995, S. 517f.) setzen sich Wechselkosten aus mehreren Kategorien zusammen, zu denen z.B. die folgenden zählen können:

- der Verlust der Kompatibilität mit bestehender Ausrüstung, wie sie bspw. bei Rasierern und Klingen, Füllfederhaltern und Tintenpatronen oder unterschiedlichen Komponenten eines Computersystems erforderlich ist;

- die mit dem Anbieterwechsel verbundenen Transaktionskosten, die einem Konsumenten bspw. beim Schließen eines Bankkontos und dem Eröffnen eines neuen Kontos 
bei einer anderen Bank oder bei der Rückgabe eines langfristig gemieteten Gegenstands und der neuen Leihe bei einem anderen Anbieter entstehen;

- die Lernkosten durch die Verwendung einer neuen Marke, wenn bspw. aufgrund der neuen Marke Prozesse verändert werden müssen;

- das Risiko geringer Qualität bei ungetesteten Marken, die bspw. Konsumenten dazu veranlasst, bei Medikamenten auf solche zurückzugreifen, von deren Wirkung sie sich bereits überzeugen konnten, anstatt neue Medikamente auszuprobieren (vgl. auch SChmalensee, 1982, S. 354);

- der Verlust angesparter Guthaben bei Kundenbeziehungsmarketingprogrammen bspw. in Form von Rabattmarken oder Vielfliegerprogrammen sowie

- psychologische Wechselkosten, die bspw. auf Gewohnheiten, Verbundenheit gegenüber dem Anbieter oder erlerntem Verhalten beruhen.

PING (1990, S. 186-189) unterscheidet zwischen vier Kategorien von Wechselkosten, die mit einem Anbieterwechsel verbunden sind: Als Folge der Aufhebung einer KundeAnbieter-Beziehung entstehen Auflösungskosten. Bei der Suche nach adäquaten alternativen Anbietern und dem Aufbau einer neuen Geschäftsbeziehung fallen Such- und Setup-Kosten in Form von ökonomischen und psychischen Aufwendungen an. Mit der Beendigung einer Geschäftsbeziehung geht ein Verlust materiellen und immateriellen Nutzens einher. Eine neue Geschäftsbeziehung aufzunehmen ist mit Risiken verbunden.

Neben den vorgestellten Aufzählungen von Wechselkosten existieren Kategorisierungen, die mit statistischen Mitteln gewonnen wurden. Üblicherweise entstammen die gewonnenen Kategorien einer Analyse mit drei Schritten: Im ersten Schritt werden Indikatoren zur Messung von bereits bekannten Wechselkostenarten entwickelt und empirische Daten für diese Indikatoren gesammelt. Mit Hilfe von exploratorischen Faktorenanalysen werden die Indikatoren im zweiten Schritt zu Indikatorgruppen umgruppiert. Im dritten Schritt erhalten die Indikatorgruppen eine inhaltliche Interpretation. Der Ablauf dieser Analyse impliziert, dass keine zusätzlichen Arten von Wechselkosten entdeckt werden. Vielmehr werden bereits bekannte Arten von Wechselkosten neu gruppiert. Einen Schwachpunkt dieses Vorgehens stellt der dritte Schritt dar, der im Extremfall zu fragwürdigen Resultaten führen kann.

Beispiele für das empirisch gestützte Vorgehen bilden die Aufsätze von JONES ET AL. (2002) sowie BURNHAM ET AL. (2003), die nachfolgend kurz vorgestellt werden. Basierend auf einer Untersuchung zahlreicher Perspektiven aus Ökonomie, Psychologie und Marketing durch Guiltinan (1989) teilen Jones ET AL. (2002, S. 442) Wechselkosten grob in 
die drei Kategorien Kontinuitätskosten, Lernkosten und versunkene Kosten ein, die sich auf insgesamt sechs Dimensionen aufspalten: Nutzenverlustkosten, Unsicherheitskosten, vor dem Wechsel liegende Such- und Bewertungskosten, nach dem Wechsel entstehende Verhaltens- und kognitive Kosten, Einrichtungskosten sowie versunkene Kosten. BURNHAM ET AL. (2003, S. 111f.) identifizieren drei Wechselkostentypen mit insgesamt acht Wechselkostenfacetten: Den ersten Wechselkostentyp bezeichnen BuRnHAM ET AL. als prozedurale Wechselkosten. Dieser Typ umfasst als Facetten die Kosten wirtschaftlichen Risikos sowie Bewertungs-, Lern- und Einrichtungskosten. Der zweite Wechselkostentyp, die finanziellen Wechselkosten, besteht aus den Wechselkostenfacetten Nutzenverlustkosten und monetäre Kosten des Wechselvorgangs. Der dritte Wechselkostentyp sind die Beziehungswechselkosten, die sich aus den Facetten persönliche Beziehungsverluste und Markenverbundenheitswechselkosten zusammensetzen.

Nicht alle der aufgeführten Wechselkostenarten sind auch für den Strommarkt für Haushaltsstromkunden relevant: Da Strom ein homogenes Produkt ist, muss man nach einem Anbieterwechsel weder den Konsum von Strom neu lernen noch irgendwelche Prozesse ändern. Kompatibilitätsprobleme sind ebenfalls ausgeschlossen: nach einem Stromanbieterwechsel müssen keine neuen Geräte angeschafft werden. Folglich lassen sich drei Wechselkostenarten identifizieren, die für den Strommarkt für Haushaltsstromkunden offensichtlich von Bedeutung sind:

- der unmittelbar mit dem Wechsel verbundene Aufwand,

- die Such- und Informationskosten, die vor einem Anbieterwechsel anfallen sowie

- das wahrgenommene Risiko, das ein Konsument einem Anbieterwechsel zuschreibt.

Die Wahl der drei Komponenten „direkter Aufwand eines Wechsels“, „Such- und Informationskosten“ sowie „wahrgenommenes Risiko“ spiegelt zum einen die von KLEMPERER (1995) als relevant erachteten Dimensionen wider. Zum anderen steht sie in Einklang mit den von BEERLI ET AL. (2004) entwickelten Indikatoren für Wechselkosten im Kontext des Wechselverhaltens bei Banken: Die Indikatoren von BeERLI ET AL. (2004, S. 262) fragen nach dem Zeitaufwand für die Informationssuche, der Anstrengung für die Anbieterwahl und dem Risiko, dass man durch den neuen Anbieter nicht zufriedengestellt wird. Die drei als relevant identifizierten Wechselkostenarten werden nachfolgend eingehender beschrieben. 


\section{Direkter Aufwand eines Wechsels}

Der Aufwand bzw. die Kosten (beide Begriffe werden in diesem Kontext synonym verwendet), die sich unmittelbar dem Wechselvorgang zuordnen lassen, werden im Folgenden als der direkte Aufwand eines Wechsels bezeichnet. Sie entsprechen der von KLEMPERER (1987a, S. 138; 1995, S. 517) als Transaktionskosten des Anbieterwechsels definierten Facette der Wechselkosten. KLEMPERER versteht darunter bspw. den Aufwand, den der Wechsel eines Telefonanbieters mit sich bringt, oder der mit der Auflösung eines Bankkontos und Eröffnung eines anderen bei einer konkurrierenden Bank verbunden ist. Speziell auf den Wechsel eines Stromanbieters bezogen sind eine Reihe von Ausprägungsformen für direkten Aufwand eines Wechsels denkbar. Dazu zählen die folgenden Beispiele:

- Der emotionale Aufwand, sich mit dem Wechsel des Anbieters beschäftigen zu müssen,

- der Zeitaufwand, der im Rahmen des Anbieterwechsels anfällt, oder

- der finanzielle Aufwand bspw. für Porto und Büromaterial.

Dieser Aufwand bildet einen Teil der gesamten mit einem Anbieterwechsel verbundenen Kosten, kurz: der gesamten Wechselkosten. In der empirischen Untersuchung von JoNES ET AL. (2000, S. 266) kristallisiert sich der einem Anbieterwechsel zugeschriebene Aufwand als das am stärksten direkt wirkende Hemmnis für einen Anbieterwechsel heraus.

\section{Such- und Informationskosten}

Suchkosten sind ein schon seit längerer Zeit bekanntes Phänomen. So spricht CoASE (1937, S. 390) im Kontext von Transaktionskosten von den „costs [...] of discovering what the relevant prices are." Die Suche und der Informationsbedarf von Konsumenten sind jedoch nicht auf den Preis beschränkt; eine ebenfalls wichtige Information ist i. A. die Qualität des Angebots (wenn auch weniger im Strommarkt für Haushaltsstromkunden). Such- und Informationskosten umfassen jeglichen empfundenen Aufwand, der mit einer möglichen Suche, Beschaffung, Analyse und Bewertung von Information verbunden ist. Bei Such- und Informationskosten kann es sich tatsächlich um finanzielle Kosten handeln, die bspw. für den Kauf von entsprechender Fachliteratur oder eine Internetrecherche anfallen. Darüber hinaus spielt der zeitliche Aufwand für die Beschaffung und Bewertung von relevanter Information eine wichtige Rolle. Nicht zuletzt verursacht die Informationssuche, die Abwägung von Alternativen und ganz allgemein die geistige Hinwendung zum Thema Anbieterwechsel einen psychischen Aufwand. 
Wie Konsumenten sich angesichts von Suchkosten verhalten, ist nicht nur Thema der Konsumentenverhaltensforschung, sondern auch der Informationsökonomie. Die Informationsökonomie als ein Teilbereich der neoklassischen Mikroökonomie (BACKHAUs ET AL., 1994, S. 33-36) geht auf den im Jahre 1961 von STIGLER veröffentlichten Aufsatz „The Economics of Information" zurück. STIGLER analysiert darin die Suche von Konsumenten nach den Preisen für ein homogenes Gut, wenn Preisstreuung und Informationskosten vorliegen. Das Modell von STigler sowie dessen Weiterentwicklungen werden heute zu den sog. Suchkostenansätzen gezählt. Diese versuchen, „das Informationsbeschaffungsverhalten von Nachfragern in Bezug auf Preise und/oder die Qualität von Gütern bei existierender Preis- bzw. Qualitätsstreuung zu beschreiben" (BAYón, 1997, S. 17). Die Arbeit von STIGLER (1961) motivierte eine Reihe anderer Autoren, das ursprüngliche Verständnis von Märkten zu modifizieren und zu erweitern. Wichtige Weiterentwicklungen lieferten insbesondere McCall (1965), Diamond (1971), Phelps und Winter (1970), Sutton (1980), Butters (1977) sowie Ungern-Sternberg (1982, 1984). Forschung auf dem Gebiet der Informationsökonomie demonstriert, dass steigende Informationskosten den Umfang von Suchverhalten reduzieren (URBAny, 1986). BAYón (1997, S. 17) weist darauf hin, dass das Informationsbeschaffungsverhalten der Nachfrager nicht auf den Fall des Erstkaufs beschränkt ist. Es ist auch bei einem Anbieterwechsel bei Versorgungsbeziehungen vorzufinden.

\section{Wahrgenommenes Risiko}

In der Konsumentenverhaltensforschung ist das Konzept des wahrgenommenen Risikos etabliert, das die Konsumentenperzeption von Unsicherheit und nachteiligen Konsequenzen eines Handelns umfasst (vgl. Dowling und Staelin, 1994, S. 119). Möchte ein Konsument bspw. seinen Anbieter wechseln, weil er durch die günstigeren Preise alternativer Anbieter einen Preisvorteil realisieren könnte, besteht für ihn Unsicherheit darüber, ob das seiner Wechselentscheidung zu Grunde liegende Preisgefüge konstant bleibt oder ob sich durch Preisänderungen der Anbieter ein Anbieterwechsel im Nachhinein als unvorteilhaft erweist. Der Konsument bewertet das Eingehen von Unsicherheit in einer Anbieterwechselentscheidung als Belastung, die er zusammen mit seinen übrigen Wechselkosten zu tragen hat (ähnlich Plinke, 2000, S. 26).

Theoretische Untermauerung findet das Phänomen der Konsumentenunsicherheit in der Risikotheorie, die auf die Arbeit von BAuER (1960) zurückgeht. Die Risikotheorie basiert auf der Annahme, dass das Kaufverhalten von Konsumenten durch deren Versuch bestimmt wird, das subjektiv wahrgenommene Risiko beim Kauf zu reduzieren (BAUER, 1960, S. 390). Subjektiv wahrgenommenes Risiko ist in diesem Zusammenhang zu verste- 
hen als,$[\ldots]$ the extent to which the consumer is uncertain about the consequences of an action [...]" (Hoyer und MAcInnis, 1997, S. 45). Wahrgenommenes Risiko entsteht dadurch, dass der Konsument aufgrund der situativ verfügbaren Information Abweichungen zwischen seinen Standards und den voraussehbaren Folgen seines Handelns registriert (ähnlich Kroeber-Riel und Weinberg, 2003, S. 251). Das Ausmaß des wahrgenommenen Risikos lässt sich in zwei Komponenten zerlegen (Cox, 1967a, S. 38):

- die Schwere der negativen Konsequenzen („amount at stake“) sowie

- die Wahrscheinlichkeit des Auftretens der negativen Konsequenzen („,feeling of subjective certainty").

In Anlehnung an Stone und Mason (1995, S. 144f.) lassen sich fünf Arten von Risiken unterscheiden, die ein Kunde wahrnehmen kann:

- funktionale Risiken, d.h. ein anderer Anbieter stellt die Versorgung nicht auf die gewünschte Weise oder gar nicht sicher,

- finanzielle Risiken, d.h. ein Anbieterwechsel war nicht lohnend oder bringt gar finanzielle Nachteile mit sich,

- gesundheitliche Risiken, d. h. ein anderer Anbieter gefährdet die körperliche Unversehrtheit des Kunden,

- soziale Risiken, d.h. ein Anbieterwechsel schadet der sozialen Stellung des Kunden, und

- psychologische bzw. psychosoziale Risiken, d.h. ein anderer Anbieter stimmt nicht mit der Selbsteinschätzung des Kunden überein.

Diese fünf Komponenten werden als funktional unabhängig voneinander gesehen (JACOBY und Kaplan, 1972, S. 382): Sollte sich eine Risiko-Komponente erhöhen, hat dies keinen Einfluss darauf, ob die anderen Komponenten sinken, gleich bleiben oder sich ebenfalls erhöhen. In empirischen Studien über viele Produktkategorien hinweg erweisen sich die fünf Risiko-Komponenten allerdings als statistisch zusammenhängend (JACOBY und KAPLAN, 1972, S. 391).

Überschreitet das vom Konsumenten subjektiv wahrgenommene Risiko eine individuelle Toleranzschwelle, wird der Konsument versuchen, das Risiko durch geeignete Risikoreduktionsstrategien auf ein akzeptables Niveau zu senken (Kroeber-Riel und WeinberG, 2003, S. 251f.). Zur Risikoreduktion stehen dem Konsumenten zahlreiche Vorgehensweisen zur Verfügung: 
- Eine in der Literatur diskutierte Strategie zur Risikoreduktion ist die Informationssuche (zustimmend z. B. Dowling und Staelin, 1994; Beatty und Smith, 1987; ablehnend z. B. GEMÜNDEN, 1985). Zusätzliche Information kann Konsumenten helfen, sich über Sachverhalte zu vergewissern.

- Insbesondere in der Bewertungsphase des Entscheidungsprozesses versuchen Konsumenten, das wahrgenommene Risiko vor allem durch persönliche Kommunikation, nicht zuletzt durch Inanspruchnahme des Rats von Meinungsführern, zu reduzieren (Kroeber-Riel und Weinberg, 2003, S. 251).

- Eine schon seit längerer Zeit bekannte Möglichkeit der Risikoreduktion liegt in loyalem Kundenverhalten (vgl. WeinBerg, 1977; Hentschel, 1991).

Aus obigen Überlegungen wird deutlich, dass das wahrgenommene Risiko eines Anbieterwechsels eher dazu führt, dass Konsumenten ihren Anbieter nicht wechseln.

\subsubsection{Hypothesen zum Einfluss der Wechselkosten bei Versorgungsbeziehungen}

Direkte Effekte der Wechselkosten auf das Wechselverhalten werden auf zweifache Weise durch Theorien gestützt: erstens durch die Austauschtheorie und zweitens durch die Theorie geplanten Verhaltens.

Gemäß der Austauschtheorie wirken sich wahrgenommene Wechselkosten auf die Höhe des Vergleichsstandards für Alternativen $\left(C L_{\text {alt }}\right)$ aus (Kelley und ThiBAut, 1978, S. 9). Angelehnt an Homans (1974, S. 31) lassen sich Wechselkosten sogar als identisch mit $C L_{\text {alt }}$ auffassen. Wechselkosten können dafür sorgen, dass es für ein Individuum B unattraktiv wird, bestimmte Alternativen zu wählen. „Making certain relationships unavailable moves down B's $C L_{a l t}$ " (ThiBaut und Kelley, 1959, S. 170). Sinkt der Vergleichsstandard für Alternativen, so akzeptiert ein Individuum auch eine schlechtere Leistung des bisherigen Anbieters, d. h. die gegenwärtige Beziehung wird eher beibehalten.

Aufbauend auf der Theorie geplanten Verhaltens lassen sich wahrgenommene Wechselkosten als ein Spezialfall wahrgenommener Verhaltenskontrolle auffassen (BANSAL und TAYLOR, 1999): Die von einem Konsumenten wahrgenommenen Wechselkosten können diesen in seiner Handlungsfreiheit einschränken. Wenn seine Einstellungen für eine bestimmte Handlung wie z. B. einen Anbieterwechsel sprechen, kann er angesichts wahrgenommener Wechselkosten davon absehen, eine entsprechende Handlungsabsicht zu entwickeln. Selbst wenn der Konsument die Absicht zum Wechseln gebildet hat, können ihn Wechselkosten möglicherweise davon abhalten, den Anbieterwechsel wirklich zu vollziehen. 
Die starke theoretische Untermauerung geht einher mit empirischer Evidenz: ANDERSON (1994) belegt empirisch einen signifikanten, aber schwachen Einfluss der Wechselkosten auf die Wiederkaufabsicht: Je höher die Wechselkosten sind, desto größer ist die Wiederkaufabsicht. Julander und SöDERLund (2003) können mit ihrer empirischen Studie einen wesentlichen direkten Einfluss der Wechselkosten aufzeigen: Demnach haben Wechselkosten einen direkten positiven Einfluss auf die Wiederkaufabsicht.

Für die Wechselkosten als Summe der direkten Wechselkosten, der Informations- und Suchkosten sowie des wahrgenommenen Risikos lässt sich die folgende Hypothese aufstellen:

\section{Hypothese 7}

Die Höhe der von einem Kunden wahrgenommenen gesamten Wechselkosten steht in gegensätzlichem Verhältnis zur Intention, den Anbieter zu wechseln.

EGGERT (1999, S. 73) fasst Wechselkosten als eine Komponente auf, die nicht in der Handlungsmacht des Individuums liegt, d. h. als eine Art von Handlungskontrolle im Sinne der Theorie des geplanten Verhaltens. Ähnlich fassen BANSAL und TAYLOR (1999, S. 201) Wechselkosten als eine unabhängige Variable auf und integrieren sie als eine Form der wahrgenommenen Verhaltenskontrolle in ihr Modell zur Erklärung des Wechselverhaltens von Hypothekenkunden. Nach dieser Auffassung dürfte sich besonders ein Einfluss der Wechselkosten auf das tatsächliche Verhalten zeigen:

\section{Hypothese 8}

Die Höhe der von einem Kunden wahrgenommenen gesamten Wechselkosten steht in gegensätzlichem Verhältnis zum Wechselvollzug.

Nachdem nun die direkten Effekte von Wechselkosten herausgearbeitet worden sind, geht der folgende Unterabschnitt auf mögliche Interaktionseffekte der Wechselkosten ein.

\subsubsection{Wechselkosten als Moderator der Wirkung der Kundenzufriedenheit}

Ökonomische Modelle des Käuferverhaltens postulieren im Allgemeinen, dass Konsumenten bei Kaufentscheidungen die Kosten und den Nutzen gegeneinander abwägen (so bspw. Hauser und Wernerfelt, 1990; RatchFord, 1982; Stigler, 1961). In Anlehnung an Jones ET AL. (2000, S. 262) lässt sich folgern, dass bei steigenden wahrgenommenen Wechselkosten diese den wahrgenommenen Nutzen aus einem Anbieterwechsel aufwiegen, der aus Unzufriedenheit mit dem Anbieter herrührt.

In Bezug auf die Wirkungsweise der Wechselkosten im Zusammenhang mit der Kundenloyalität haben die meisten einschlägigen Arbeiten bisher die Wechselkosten als einen 
Moderator der Beziehung zwischen Kundenzufriedenheit und Kundenloyalität angesehen (Yang und Peterson, 2004, S. 805). So merken bspw. Jackson (1985) sowie Porter (1980) an, dass substanzielle Wechselkosten oder besonders mühsame Wechselprozesse selbst unzufriedene Kunden dazu bewegen, die Beziehung mit ihrem Anbieter beizubehalten und von einem Anbieterwechsel abzusehen.

Jones ET AL. (2000, S. 262) formulieren den moderierenden Einfluss der Wechselkosten auf die Wirkbeziehung zwischen Kundenzufriedenheit und Kundenbindung dergestalt, dass „[...] when perceived switching costs are low, dissatisfied consumers should be more likely to defect than are satisfied customers. Alternatively, when perceived switching costs are high, customers may remain despite their dissatisfaction due to perception that switching costs outweigh switching benefits." Viele Wissenschaftler schreiben Wechselkosten eine Schlüsselbedeutung als moderierende Variable zu, die die Kundenloyalität durch die Kundenzufriedenheit beeinflusst (vgl. Fornell, 1992; LeE ET AL., 2001; Oliver, 1999).

Empirische Studien aus der Marketingwissenschaft und der Mikroökonomie sind allerdings zu durchaus widersprüchlichen Ergebnissen hinsichtlich der Wirkweise von Wechselkosten auf die Kundenloyalität gelangt (VIARD, 2002): Einerseits konnte die Marketingwissenschaft bereits in vielen Bereichen signifikante Moderatoreffekte der Wechselkosten aufdecken. LEE ET AL. (2001), die bspw. den Effekt von Wechselkosten auf die Kundenzufriedenheits-Kundenloyalitäts-Wirkbeziehung im französischen Mobilfunkmarkt untersuchten, fanden den Moderatoreffekt signifikant bestätigt. HAUSER ET AL. (1994) haben entdeckt, dass substanzielle Wechselkosten Kunden weniger empfindsam für wahrgenommene Kundenzufriedenheitsniveaus werden lassen. Zum gleichen Ergebnis gelangten Anderson und Sullivan (1993) im Bankensektor. Auch eine Untersuchung von Sharma und PATterson (2000) im Bereich der privaten Vermögensberatung deutet darauf hin, dass Wechselkosten die Wirkbeziehungen von Kundenvertrauen und Kundenzufriedenheit auf das Kundencommitment positiv moderieren. Anderson (1994, S. 28) kann bei einer tiefer gehenden Analyse von Daten aus dem Swedish Customer Satisfaction Barometer empirisch stützen, dass bei hohen Wechselkosten die Wiederkaufabsicht von Konsumenten weniger sensibel auf Schwankungen der Kundenzufriedenheit reagiert. Jones ET AL. (2000, S. 262) können einen Interaktionseffekt empirisch belegen und gelangen dabei zu folgender Interpretation: Erst wenn die Kundenzufriedenheit unter ein bestimmtes Niveau sinkt, beginnen Konsumenten, Wechselkosten in Betracht zu ziehen oder allgemein von Wechselkosten beeinflusst zu werden. Julander und SöDERLund (2003) können mit ihrer Studie u. a. einen wesentlichen moderierenden Einfluss der Wechselkosten aufzeigen: Demnach verringert sich der Einfluss der Kundenzufriedenheit auf die Wiederkaufabsicht, wenn die Wechselkosten hoch sind. Andererseits zeigt sich der 
Interaktionseffekt nicht durchgängig: So können bspw. BuRnHAM ET AL. (2003) keinen signifikanten Interaktionseffekt der Wechselkosten auf die Wirkbeziehung zwischen der Kundenzufriedenheit und der Wechselabsicht finden. Eine mögliche Erklärung für diese divergierenden empirischen Ergebnisse liefert NiELSON (1996): Der moderierende Effekt der Wechselkosten auf die Wirkbeziehung zwischen Kundenzufriedenheit und Kundenloyalität hängt von situativen Variablen wie z. B. der Branche, den Produkten oder den Kunden ab und ist nicht in allen Fällen signifikant.

Die obigen Betrachtungen zu einem hypothetischen moderierenden Effekt der Wechselkosten münden in die folgenden beiden Hypothesen. Die Hypothese 9 bezieht sich auf den moderierenden Einfluss der Wechselkosten auf die Beziehung zwischen Kundenzufriedenheit und Wechselabsicht:

\section{Hypothese 9}

Je geringer die wahrgenommenen Wechselkosten sind, desto eher führt die Unzufriedenheit eines Kunden mit seinem Anbieter zur Absicht, den Anbieter zu wechseln.

Die Hypothese 10 bezieht sich auf den moderierenden Einfluss der Wechselkosten auf die Beziehung zwischen Kundenzufriedenheit und Wechselvollzug:

\section{Hypothese 10}

Je geringer die wahrgenommenen Wechselkosten sind, desto eher führt Unzufriedenheit eines Kunden mit seinem Anbieter dazu, dass der Kunde den Anbieter wechselt.

\subsection{Involvement als Determinante des Wechselverhaltens}

Das Involvementkonzept wird seit nahezu vier Jahrzehnten in der Konsumentenverhaltensforschung untersucht; in der Psychologie lassen sich die Ursprünge sogar noch weiter zurückverfolgen (MunCY und HunT, 1984): Mit der Theorie des Ego-Involvements brachten SHERIF und CANTRIL (1947) den Begriff „Involvement“ erstmals in die wissenschaftliche Diskussion. Krugman $(1965,1966)$ machte ihn in der Marketingwissenschaft populär, indem er das Fernsehen als „Low-Involvement-Medium“ identifizierte.

Kaum ein Konstrukt der Konsumentenverhaltensforschung hat in den vergangenen 25 Jahren so viel Aufmerksamkeit erhalten wie das Involvement. Es „hat sich inzwischen zu einem der bedeutendsten Erklärungsansätze der Konsumentenforschung entwickelt" (Kuss und Tomczak, 2000, S. 64). Involvement kann möglicherweise auch einen wichtigen Erklärungsbeitrag zum Wechselverhalten bei Versorgungsbeziehungen leisten. Gestützt wird diese These vor allem durch die empirischen Ergebnisse von BAKAY (2003). Dieser Ab- 
schnitt arbeitet heraus, was unter Involvement zu verstehen ist und auf welche Weise Involvement das Wechselverhalten bei Versorgungsbeziehungen beeinflusst.

Bis heute existiert weder Konsens darüber, was genau unter Involvement zu verstehen ist, noch wie dieses Konstrukt konzeptualisiert und operationalisiert werden soll (vgl. beispielhaft Muncy und Hunt, 1984; Park und Mittal, 1985; Ratchford, 1987; Zaichkowsky, 1985). Die unbefriedigende Situation in der Involvementforschung beschreibt MitTAL (1995, S. 664): „[R] esearchers have invoked somewhat divergent images of the construct." So existiert einerseits eine Vielzahl an teilweise in sich plausiblen Involvementdefinitionen, die aber aufgrund von fehlender oder unzureichender Abgrenzung ein Phänomen beschreiben, das verhaltens- und sozialpsychologisch synonym ist mit Begriffen wie „Interesse“, „wahrgenommener Relevanz" oder „Commitment" (MitTal, 1982, S. 15-22). Unabhängig von der Fragestellung, ob die gesamte Erforschung und Konzeptualisierung des Involvementkonstrukts grundsätzlich einen Zugewinn für die Marketingforschung darstellt, erscheint es generell unzweckmäßig und nur zur allgemeinen Verwirrung beitragend, bereits vorhandene Konzepte unter anderem Namen neu zu entwickeln. Zwar ist häufig intuitiv und unter Berücksichtigung des jeweiligen Kontextes ersichtlich, welches Phänomen durch Involvement beschrieben werden soll. Eine allgemein gültige Bedeutung hat das Involvement jedoch noch nicht erhalten. Insbesondere herrscht Uneinigkeit über das Bezugsobjekt des Involvements: Handelt es sich bei Involvement um eine Eigenschaft eines Mediums bzw. einer Produktart? Ist Involvement eine Eigenschaft eines Individuums, die sich in einem geistigen Zustand erhöhten Interesses für eine spezielle Produktart äußert? Oder bezeichnet Involvement das durch diesen Zustand ausgelöste Informationsverhalten für eine Produktart?

Marketer tendierten i. d. R. dazu, den Aspekt der kognitiven Auseinandersetzung des Konsumenten (im Sinne der Einstellungsbildung) mit dem Produkt bzw. der Werbebotschaft des Produktes hervorzuheben und ihm die größte Relevanz bezüglich zu ergreifender Marketingstrategien zuzugestehen. Grundsätzlich wurde von einem „hoch-involvierten“ Konsumenten ausgegangen, der sich uniform intensiv und rational mit wahrgenommenen Werbebotschaften auseinander setzt und diese daraufhin mit seinen persönlichen Einstellungen abgleicht, bevor er eine Kaufentscheidung trifft (Mittal, 1982, S. 5). Anders als der kognitiven Komponente wurde der affektiven Komponente typischerweise eine untergeordnete Rolle zugewiesen, möglicherweise aufgrund ihrer nur bedingt verallgemeinerbaren und kontrollierbaren Natur. Ursache für dieses Vorgehen ist mitunter auch die Bewahrung des traditionellen Schemas, das von einem grundsätzlich interessierten und engagierten Konsumenten ausgeht - unabhängig davon, wie viel Interesse der Konsument dem speziellen Produkt beimisst. Wilkie (1994, S. 224) merkt dazu an, dass „,...] traditional 
models such as the hierarchy of effects have built-in assumptions of high CI [Consumer Involvement]." Zwischenzeitlich hat sich jedoch die Einsicht entwickelt, dass die Höhe des Involvements einen entscheidenden Einfluss auf das Verhalten von Konsumenten hat. Dieser Verständniswandel hatte insbesondere einen signifikanten Einfluss auf den Bereich der Konsumentenforschung und führte zu einer Erweiterung der bestehenden Modelle um den Bereich des low-involvierten Konsumenten. Die sich daraus ergebenden typischen highund low-involvierten Konsumentenverhaltensweisen veranschaulicht die Tabelle 4.2.

Um das Involvement in ein Modell des Wechselverhaltens bei Versorgungsbeziehungen zu integrieren, ist zunächst festzulegen, welche der vorhandenen Konzeptualisierungen sich am besten für die weitergehende Analyse eignet. Daher beschäftigt sich Unterabschnitt 4.4.1 mit den bestehenden Forschungslinien und gelangt zu einer Definition, die für diese Arbeit zweckmäßig ist. Unterabschnitt 4.4 .2 arbeitet mögliche direkte Wirkungen des Involvements im Rahmen des Wechselverhaltens bei Versorgungsbeziehungen heraus, und Unterabschnitt 4.4.3 bringt mögliche Interaktionseffekte des Involvements zur Sprache.

\subsubsection{Forschungslinien und Definition}

Die Ziele der Involvementforschung für das Marketing liegen darin, das von Einstellungen beeinflusste kognitive Konsumentenverhalten komplexer und realistischer abzubilden und somit insbesondere auch die Werbeeffektivität und das Entscheidungsverhalten von gering-involvierten Konsumenten besser messen und verstehen zu können. Ein weiteres Feld der Involvementforschung sind die marketingstrategischen Implikationen, die man aus den Erkenntnissen über das Informationsverhalten bezüglich unterschiedlicher Produktarten gewinnt.

Eine gängige Praxis ist es, Konsumgüter mit der Eigenschaft des Involvementpotenzials zu belegen und in die beiden dichotomen Klassen der „High-“ und „Low-InvolvementProdukte" einzugliedern. Als klassische Produkte der High-Involvement-Kategorie gelten beispielsweise Autos, Mode und Elektrogeräte, wohingegen Zahnpasta, Salz oder Haushaltspapier in der Regel als „Low-Involvement-Produkte“ bezeichnet werden. Die Grenze zwischen High- und Low-Involvement-Produkten verläuft parallel zu den beiden Produktkategorien „specialty goods“ und „convenience goods“. Aus diesem Umstand geht implizit hervor, dass neben der individuell wahrgenommenen Relevanz des Konsumenten für ein Objekt (in diesem Falle ein Produkt) auch die objektive und subjektive Differenzierungsmöglichkeit der Produkte innerhalb einer Produktart direkten Einfluss auf das Involvement ausübt. Vorwiegend unterscheiden sich die bestehenden Involvementkonzeptualisierungen in viererlei Hinsicht: 


\section{Traditional, High Involvement View Newer, Low Involvement View of a of an Active Consumer Passive Consumer}

Consumers are information processors.

Consumers learn information at random.

Consumers are information seekers.

Consumers are information gatherers.

Consumers represent an active audience Consumers represent a passive audience for advertising. As a result, the effect of advertising on the consumer is weak. for advertising. As a result the effect of advertising on the consumer is strong.

Consumers evaluate brand beliefs before buying.

Consumers buy first. If they evaluate brands, it is done after the purchase.

Consumers seek to maximize expected satisfaction.

Personality and life-style are connected to consumer behavior because the product is closely tied to the consumer's identity and belief system.

Reference groups influence consumer behavior because of the importance of the product to group norms and values.

Consumers seek some acceptable level of satisfaction.

Personality and life-style are not connected to consumer behavior because the product is not closely tied to the consumer's identity and belief system.

Reference groups exert little influence on product choice because products are unlikely to be related to group norms and values.

Tabelle 4.2: Vergleich der Sicht von hoch und gering involvierten Konsumenten (vgl. ASSAEL, 1992, S. 104)

- Das Bezugsobjekt des Involvements kann variieren. Es kann eine Person, ein Medium, ein Produkt oder auch etwas ganz anderes sein.

- Die Ausprägungsformen des Involvements können kontinuierlich oder diskret sein.

- In Bezug auf Dynamik kann Involvement einen Prozess oder einen Zustand beschreiben.

- Das Involvement kann eine unterschiedliche Position im kausalen Wirkungsbund annehmen, d. h. man kann das Involvement als Ursache, Zustand oder Wirkung im Informations- und (Kauf-)Entscheidungsprozess des Konsumenten ansehen.

Das Konstrukt Involvement lässt sich weiter unterteilen in zwei Typen von Involvement: beständiges Involvement („enduring involvement“) und Situationsinvolvement („situational involvement“) (Houston und Rothschild, 1978, S. 184). Mittal und Lee (1988, 
S. 43) sprechen von „product involvement“ und „brand-choice involvement“, KANTHER (2001, S. 81) bevorzugt die Begriffe „Produktinvolvement“ und „Kaufinvolvement“. Unter beständigem Involvement ist die anhaltende persönliche Wichtigkeit eines Produkts bzw. das permanente Interesse an einem Produkt - unabhängig von situativen Einflüssen $\mathrm{zu}$ verstehen. Als situatives Involvement wird das kurzzeitig erhöhte Interesse an einem Produkt oder die temporäre persönliche Wichtigkeit eines Produkts bezeichnet, die auf vorübergehenden Umständen wie bspw. einem Kauf oder einer Kontaktierung durch einen Anbieter beruhen.

Das Involvement besitzt eine starke Verwandtschaft mit einer Einstellung zu einem Objekt, die in der Einstellungs- bzw. Verhaltensliteratur als unabhängige Variable angesehen wird (SNYDER, 1982). Die Involvementforschung ist sich im Großen und Ganzen einig, dass das Involvement verwandt oder sogar identisch mit der wahrgenommenen Wichtigkeit des Stimulus ist (MitTal, 1995, S. 664). Ein Stimulus kann hierbei das Produkt selbst oder die Kaufentscheidungsaufgabe sein. In einer Literaturdurchsicht gelangen GrEENWALD und LEAVITT (1984, S. 583) zu dem Schluss, dass „, [t]here is a consensus that high involvement means (approximately) personal relevance or importance." Ist ein Objekt für den Konsumenten unwichtig, so zeigt der Konsument kein Involvement - er steht dem Objekt gleichsam gefühllos oder gleichgültig gegenüber (MitTAL, 1995, S. 664).

Die empfundene Wichtigkeit eines Produkts oder einer Produktklasse entspricht jedoch in der in dieser Arbeit untersuchten Branche möglicherweise nicht dem diskutierten Involvementbegriff: Den meisten Kunden ist die Abhängigkeit von Strom und die daraus resultierende Wichtigkeit der Stromversorgung durchaus gegenwärtig; ein Leben ohne Strom ist in der heutigen Kulturgesellschaft undenkbar. Eine gedankliche Auseinandersetzung mit dem Bezug von Strom ist jedoch kaum anzutreffen, sodass BAKAY (2003) sogar allgemein von einem Low-Involvement-Produkt spricht. BAKAY schließt sich insofern der Auffassung einiger Autoren an, die Involvement nicht als verhaltenswissenschaftliches Konstrukt betrachten und somit auch nicht auf die einzelnen Kunden beziehen, sondern vielmehr als objektive Eigenschaft eines Produkts oder einer Produktkategorie interpretieren (vgl. u. a. Churchill und Suprenant, 1982, S. 493; Gotlieb et Al., 1992, S. 195f.; Patterson, 1993, S. 450f.). Gemäß dieser Sichtweise führen spezielle Produkteigenschaften zu hohem bzw. niedrigem Involvement bei allen Kunden. Für die vorliegende Arbeit ist diese Auffassung allerdings nicht hilfreich, da sie lediglich eine branchenübergreifende Varianz des Involvements unterstellt, und nicht die Varianz des Involvements zwischen den einzelnen Kunden berücksichtigt. Dass die Projektion von wahrgenommener Wichtigkeit auf das Involvement keine konstante Funktion und stark abhängig vom kognitiven Leistungsvermögen des Individuums ist, ist ein weiteres Indiz für die Notwendigkeit der 
Trennung der beiden Konzepte. Einerseits ist es denkbar, dass eine Person trotz einer hohen empfundenen Wichtigkeit für eine Produktart relativ gering involviert ist. Dies kann insbesondere vorkommen, wenn der Fokus der Relevanz auf dem funktionalen Wert liegt. Andererseits ist es eher unwahrscheinlich, dass eine Person ein hohes Involvement für eine bestimmte Produktkategorie besitzt, ohne diese zugleich für sich als relevant zu erachten. Als kausaler Zusammenhang lässt sich somit lediglich feststellen, dass eine hohe dauerhaft wahrgenommene Wichtigkeit ein notwendiges, aber nicht hinreichendes Kriterium für ein hohes dauerhaftes Involvement darstellt. Ähnlich sehen dies auch BLOCH und Richins (1983, S. 73): "The perception of product importance [..] represents a cognitive state of awareness or realization. Product Involvement [.. . ] may be thought of as the motivational state that results from the stimulus of product importance perceptions."

Die erwähnten Probleme treten nicht auf, wenn das Involvement gemäß der Definition von GLYNn und Goldsmith konzeptualisiert wird. Diese definieren Involvement als „[... ] feelings of interest, enthusiasm, and excitement that consumers have about specific product categories“ (Glynn und Goldsmith, 1993, S. 357). Hieraus ergibt sich, dass das Involvement als eine Eigenschaft jedes einzelnen Kunden anzusehen ist. Situationsabhängige Einflüsse im Sinne eines Situationsinvolvements werden nicht betrachtet. Vielmehr handelt es sich beim Involvement um ein stabiles Einstellungsmuster eines Kunden (vgl. auch Richins und BLOCH, 1991, S. 147). Dieses auch als dauerhaft (engl.: enduring) bezeichnete Involvement "[...] is the ongoing level of product concern independent of situational influences" (SHAfFer und SHerrell, 1997, S. 265). In diesem Sinne beinhaltet das Involvementkonstrukt die aktive innere Beteiligung des Konsumenten als „das innere Engagement, mit dem sich jemand einem Gegenstand oder einer Aktivität zuwendet" (Kroeber-Riel, 1991, S. 98), oder den „Aktivierungszustand, der den Nachfrager zu einer gedanklichen oder emotionalen Auseinandersetzung mit dem Gegenstand anregt" (Höser, 1998, S. 94). Heslin und Johnson (1992, S. 210) stellen heraus, dass Konsumenten , ,. .] spend time learning about a product because they are interested in, that is, involved with, it." In der Literatur wird daher übereinstimmend berichtet, dass ein hohes Produktinvolvement von Kunden mit einem hohem Wissen über das Produkt einhergeht (vgl. bspw. Celsi und Olson, 1988; Hoyer und Ridgway, 1984 sowie Mano, 1997).

Im Rahmen der vorliegenden Arbeit beschreibt Involvement in Anlehnung an die Definition von Kroeber-RIEL das innere Engagement, mit dem sich eine Person dauerhaft dem Bezug des Produkts Strom zuwendet. Es handelt sich hierbei um einen geistigen Zustand von Individuen, der kontinuierliche Werte annehmen kann. Im kausalen Wirkungskontext wird angenommen, dass das Involvement eine Ursache innerhalb des Informations- und (Kauf-)Entscheidungsprozesses von Konsumenten ist. 


\subsubsection{Hypothesen zum direkten Einfluss des Involvements innerhalb des Wechselverhaltens}

Das Involvement gilt als ein wichtiger Einflussfaktor auf das Wechselverhalten. So empfehlen Ganesh ET AL. (2000, S. 66), das Involvementkonstrukt bei der Analyse von Wechselverhalten zu berücksichtigen, denn „,..] involvement has been shown to play a key role in this area of research." Allerdings werden dem Involvement in der Literatur sich widersprechende direkte Wirkungen zugeschrieben: Einerseits führt ein höheres Involvement zu größerer Kundenloyalität (vgl. z. B. GIERING, 2000), andererseits fördert es jedoch das Wechselverhalten (vgl. z. B. BAKAY, 2003). Im Folgenden werden beide Forschungslinien vorgestellt, um aus ihnen jeweils Hypothesen zu den direkten Effekten des Involvements auf das Wechselverhalten von Konsumenten bei Versorgungsbeziehungen abzuleiten.

Die erste Forschungslinie vertreten z. B. Oliva ET AL. (1992). Sie halten allgemein einen Käufer für umso loyaler, je involvierter er ist (OLIVA ET AL., 1992, S. 85). Es bestehen diverse Ansätze, die Involvement mit Brand Commitment bzw. Markenloyalität in direktem Zusammenhang modellieren. BEATTY ET AL. (1988) zeigen, dass Involvement eine wichtige Rolle bei der Entwicklung des Commitments von Konsumenten spielt. MCQUARRIE und Munson (1987) betrachten z. B. Brand Commitment als Eigenschaft bzw. Folge des Involvements oder allgemein als positiv mit dem Involvement korrelierendes Konzept. Bloemer und Kasper (1995) entdecken bei einer Untersuchung der Markentreue bei schnelldrehenden Konsumgütern, dass das Involvement deutlich positiv mit der Kundenloyalität in Beziehung steht. GANEsh ET AL. (2000) kamen bei einer Untersuchung des Kundenstamms in der Bankbranche zu dem Ergebnis, dass Kunden, die der betrachteten Bank seit Anbeginn die Treue halten, ein höheres Involvement aufweisen als Kunden, die von einer anderen Bank zu dieser Bank gewechselt sind. Die herrschende Meinung in der Konsumentenverhaltensforschung geht maßgeblich auf BEATTY ET AL. (1988) sowie Crosby und Taylor (1983) zurück und lässt sich am besten mit den Worten von Burton und Netemeyer (1992, S. 145) wiedergeben: „Consumer behavior researchers argue that involvement with the product leads to commitment or brand loyalty in which the brand choice is stable across situations." In der deutschsprachigen Marketingliteratur ist es insbesondere GIERING (2000), die diese Forschungslinie aufgreift und später empirisch bestätigt, dass hoch involvierte Kunden eher ihrem Anbieter treu bleiben (ebenda, S. 176).

Bezogen auf das Wechselverhalten von Konsumenten in Versorgungsbeziehungen lassen sich unter Berücksichtigung der oben diskutierten Literatur die folgenden beiden Hypothesen aufstellen: 


\section{Hypothese 11}

Je höher das Involvement eines Konsumenten ist, desto niedriger ist seine Absicht, den Anbieter zu wechseln.

\section{Hypothese 12}

Je höher das Involvement eines Konsumenten ist, desto unwahrscheinlicher vollzieht er einen Anbieterwechsel.

Die zweite Forschungslinie vertritt eine gegensätzliche Auffassung zur direkten Wirkung des Involvements im Rahmen des Wechselverhaltens von Konsumenten, nämlich dass ein höheres Involvement mit ausgeprägterem Wechselverhalten einhergeht. Diese Forschungslinie erhält sowohl konzeptionelle als auch empirische Unterstützung.

Konzeptionell sprechen zwei Argumentationen gegen die oben aufgestellten Hypothesen $\mathrm{H}_{11}$ und $\mathrm{H}_{12}$ : Erstens mögen die Hypothesen zwar bei Konsumenten zutreffen, die aufgrund eines hohen Involvements einen von ihnen als optimal angesehenen Anbieter gefunden haben. Solche Konsumenten stellen jedoch bei der gegenwärtigen Situation im Strommarkt für Haushaltsstromkunden die Minderheit dar. Die Mehrheit der Kundenbeziehungen ist durch die geografische Zugehörigkeit $\mathrm{zu}$ einem vormals monopolistisch bedienten Versorgungsgebiet bedingt und beruht nicht auf der Auswahl des womöglich besten Stromanbieters. Zweitens ist ein gewisser Grad an Involvement Voraussetzung dafür, dass ein Konsument einen Anbieterwechsel vollziehen kann. Insofern dürfte sich ein positiver Zusammenhang zwischen Involvement und Wechselverhalten abzeichnen.

Empirische Unterstützung leistet die Arbeit von Goodman ET AL. (1995). Goodman ET AL. kommen zu dem Ergebnis, dass Kunden mit höherem Involvement eher kritisch gegenüber Fehlern eines Dienstleistungsanbieters sind und zu größerer Unzufriedenheit neigen als Kunden mit niedrigerem Involvement. Am einschlägigsten ist das empirische Ergebnis von BAKAY (2003, S. 175f.) zur Kundenbindung von Haushaltstromkunden: BAKAY kann einen starken positiven Zusammenhang zwischen Produktinvolvement und Wechselbereitschaft belegen.

Konkurrierend zu den oben aufgestellten Hypothesen $\mathrm{H}_{11}$ und $\mathrm{H}_{12}$ lassen sich darum auch die folgenden beiden Hypothesen bilden:

\section{Hypothese 13}

Je höher das Involvement eines Konsumenten ist, desto höher ist seine Absicht, den Anbieter zu wechseln.

\section{Hypothese 14}

Je höher das Involvement eines Konsumenten ist, desto eher vollzieht er einen Anbieterwechsel. 
In Anbetracht der konkurrierenden Hypothesen ist es im Falle des direkten Einflusses des Involvements auf das Wechselverhalten bzw. die Wechselabsicht besonders wichtig, durch eine empirische Untersuchung Klarheit über die Wirkweise zu erlangen. Aus der bestehenden Literatur lässt sich nicht eindeutig schließen, ob Involvement nun für das Wechselverhalten förderlich, hinderlich oder durch sich aufhebende Effekte letztlich unerheblich ist.

\subsubsection{Hypothesen zu Interaktionseffekten des Involvements innerhalb des Wechselverhaltens}

Dem Involvement werden neben den direkten Wirkungen auch zahlreiche moderierende Effekte zugeschrieben. Einen moderierenden Einfluss des Involvements auf die Bildung von Einstellungen konnten bspw. PetTy Et AL. (1981, S. 851f.) nachweisen. PetTy und Cacioppo (1979, S. 1923f.) leiten aus mehreren Experimenten ab, dass ein hohes Involvement mit einer Sache eine erhöhte Nachrichtenverarbeitung bewirkt und die Akzeptanz einer Nachricht entweder erhöht oder verringert. PETTY ET AL. (1983, S. 141) fanden heraus, dass Einstellungen - und dazu lässt sich nach der hier gewählten Konzeptualisierung auch die Kundenzufriedenheit zählen (s. S. 51 in Verbindung mit S. 48) Verhaltensabsichten besser vorhersagen, wenn das Involvement hoch ist. Involvement hat aber nicht nur Einfluss auf die Bildung und die Wirkung von Einstellungen, sondern beeinflusst den kompletten Kaufentscheidungsprozess von Konsumenten: „Depending on their level of involvement, consumers will differ greatly in the extensiveness of their purchase decision process (indicated by the extent of information search, the number of attributes used to compare brands, the length of the choice process, and the willingness to reach a maximum or a threshold level of satisfaction)" (LAURENT und KAPFERER, 1985, S. 42). Die Intensität des Entscheidungsprozesses ist in mehrfacher Hinsicht verhaltensrelevant (Coupey Et AL., 1998, S. 459-461). Kunden, die über ein umfangreiches Wissen über das Produkt bzw. die Produktkategorie verfügen, verlassen sich stärker auf ihr eigenes Zufriedenheitsurteil, so die Ergebnisse von Duhan et AL. (1997), Richins und Bloch (1991) sowie Spence und Brucks (1997). Schließlich gilt nach BloEmer und KASPER (1995, S. 136), dass „, [t] greater the amount of elaboration upon the brand choice, the more manifest the satisfaction."

Das Involvement beinflusst sowohl Vergleichsstandards vor einer Kaufentscheidung als auch Bewertungen nach dem Kauf. Bolfing und WoodrufF (1988, S. 21f.) entdeckten, dass Konsumenten mit hohem Involvement auch höhere Vergleichsstandards zu besitzen scheinen als Konsumenten mit geringem Involvement. Zu einem ähnlichen Ergebnis gelangten Oliver und BeARDEN (1983), die herausfanden, dass ein hohes Involvement 
bezogen auf Appetitzügler mit erhöhten Erwartungen vor der Produktverwendung einhergeht. Die Wirkung des Involvements auf Bewertungen nach dem Kauf untersuchten Gronhaug (1977) sowie Swan und Trawick (1979): Gronhaug (1977, S. 162) schloss aus empirischen Ergebnissen, dass, je höher das Involvement ist, desto extremer die Ergebnisse dieser Evaluierungen sind. SwAN und TrawiCK (1979, S. 58) verglichen, inwieweit Konsumenten nach dem Kauf eines bestimmten Produktes eine Produktevaluierung vornahmen. Während bei Kinotickets, also typischen High-Involvement-Produkten, $87 \%$ der befragten Personen angaben, eine Produktevalierung durchgeführt zu haben, bewerteten lediglich $40 \%$ bzw. $29 \%$ im Nachhinein die Produkte Fleisch bzw. Brot, die eher LowInvolvement-Produkte sind.

Manchmal kann es für Konsumenten kompliziert sein, Erwartungen zu bilden, die erhaltene Leistung zu bewerten und die Erwartungen mit der erhaltenen Leistung so zu vergleichen, als handele es sich um zwei unabhängige Elemente (BLOEMER und KASPER, 1995, S. 315). Wenn bspw. ein Konsument über ein Produkt kaum Bescheid weiß oder über keine Erfahrungen mit Alternativen (die ihm als Referenzpunkt dienen könnten) verfügt und ihm daher die Motivation und die Fähigkeit zu einer expliziten Berechnung des Unterschieds zwischen den Erwartungen und der erhaltenen Leistung fehlten, gestaltet sich ein Vergleich für ihn schwierig. Selbst wenn der Konsument dazu willens und in der Lage ist, können ihn fehlende wahrgenommene Differenzen zwischen den unterschiedlichen Angeboten von einem expliziten Vergleich abhalten (AssAEL, 1992).

Angelehnt an die Konzeptualisierung der Kundenzufriedenheit durch BLOEMER und DE RuYter (1998, S. 501) lässt sich in Abhängigkeit vom kognitiven Aufwand des Soll-/Ist-Vergleiches zwischen latenter und manifester Kundenzufriedenheit unterscheiden: Manifeste Kundenzufriedenheit liegt vor, wenn ein bewusster Vergleich zwischen der erwarteten und der tatsächlichen Leistung erfolgt. Sie lässt sich als das Ergebnis eines Vergleichs auffassen, mit dem man sich intensiv beschäftigt hat: „Manifest satisfaction is the outcome of the explicit subjective evaluation that the chosen alternative (brand) meets or exceeds the expectations" (BloEmer und KASPER, 1994, S. 153). Im anderen Fall spricht man von latenter Kundenzufriedenheit (Bloemer und Poiesz, 1989, S. 45). Latente Kundenzufriedenheit ist das Resultat einer impliziten Beurteilung, d.h. einer, mit der man sich nicht auseinander gesetzt hat: „Latent satisfaction is the outcome of the implicit subjective evaluation that the chosen alternative (brand) meets or exceeds the expectations“ (Bloemer und Kasper, 1994, S. 153). Da der Grad der Auseinandersetzung schwanken kann, ist der Unterschied zwischen manifester Kundenzufriedenheit und latenter Kundenzufriedenheit nicht absolut; vielmehr lassen sich beide als die Endpunkte eines Kontinuums ansehen (Bloemer und KAsper, 1995, S. 315). Daher plädieren 
Bloemer und Kasper (1995, S. 316, 318) dafür, Involvement als eine die Kundenzufriedenheit moderierende Variable zu modellieren, um auf diese Weise ein kontinuierliches Spektrum der Kundenzufriedenheit mit den Endpunkten manifester und latenter Kundenzufriedenheit zu erzeugen. Ist das Involvement hoch, liegt manifeste Kundenzufriedenheit oder - unzufriedenheit vor. Bei niedrigem Involvement handelt es sich um latente Kundenzufriedenheit oder -unzufriedenheit.

Zwar wurde in der Marketingforschung noch nicht der moderierende Einfluss des Involvements auf die Wechselabsicht und den Wechselvollzug untersucht. Wohl aber liegen mit den Arbeiten von Oliva et AL. (1992) und Bloemer und Kasper (1995) empirische Belege für den moderierenden Einfluss des Involvements auf die Wirkbeziehung zwischen Kundenzufriedenheit und Kundenloyalität vor. Oliver (1997) merkt an, dass die Abweichung der erwarteten von der erhaltenen Produktleistung, die sog. Diskonfirmation, unter hohem Involvement am stärksten ausgeprägt ist.

Aus den obigen Überlegungen lässt sich ableiten, dass die negative Beziehung zwischen der manifesten Kundenzufriedenheit und der Intention, den Anbieter zu wechseln, betragsmäßig größer ist als die negative Beziehung zwischen der latenten Kundenzufriedenheit und der Intention, den Anbieter zu wechseln. Das heißt:

\section{Hypothese 15}

Je höher das Involvement eines Konsumenten ist, desto stärker ist der Einfluss der Kundenzufriedenheit auf die Wechselabsicht.

Analog ist zu vermuten, dass die negative Beziehung zwischen der manifesten Kundenzufriedenheit und dem Wechselvollzug betragsmäßig größer ist als die negative Beziehung zwischen der latenten Kundenzufriedenheit und dem Wechselvollzug. Mit anderen Worten:

\section{Hypothese 16}

Je höher das Involvement eines Konsumenten ist, desto stärker ist der Einfluss der Kundenzufriedenheit auf den Wechselvollzug.

Das Involvement beeinflusst nicht nur die Wirkung von Einstellungen, sondern stellt allgemein eine wichtige Determinante des menschlichen Entscheidungsverhaltens dar. Es liegt somit nahe, dass es noch weitere moderierende Einflüsse des Involvements im Rahmen des Wechselverhaltens von Konsumenten gibt. Daher soll auch ein etwaiger moderierender Einfluss des Involvements auf die Wirkungen der übrigen bisher betrachteten Antezedenzien von Wechselabsicht und Wechselvollzug, d.h. der Wechselkosten und der Attraktivität der Alternativen, untersucht werden. 
Ähnlich der Unterscheidung zwischen latenter und manifester Kundenzufriedenheit lässt sich auch zwischen latenten und manifesten Wechselkosten differenzieren: Als latent werden Wechselkosten bezeichnet, die mit einem geringen Involvement einhergehen. Von manifesten Wechselkosten ist dann auszugehen, wenn Wechselkosten mit einem hohen Involvement zusammenfallen.

BeErli ET AL. (2004, S. 263) betten das Involvement als eine Dimension in ein weiter gefasstes Konstrukt „degree of elaboration“ ein, das sich grob als „Sorgfalt der Auswahl“ übersetzen lässt. Sie überprüfen u. a., ob die Sorgfalt der Auswahl die Wirkbeziehungen zwischen Wechselkosten und Kundenloyalität moderiert. Zwar können sie im Bankensektor keinen signifikanten moderierenden Effekt nachweisen, doch angesichts eines möglichen $\beta$-Fehlers in ihrer Untersuchung sollte diese Hypothese im Rahmen des Wechselverhaltens von Haushaltsstromkunden erneut überprüft werden.

Die Wirkweise von Wechselkosten allgemein wurde in den Hypothesen $\mathrm{H}_{7}$ und $\mathrm{H}_{8}$ formuliert. Für unterschiedliche Ausprägungen des Involvements ist zu vermuten, dass sich die Wirkstärke der Wechselkosten ändert. Dabei dürften manifeste Wechselkosten die Wechselabsicht bzw. den Wechselvollzug stärker hemmen als latente Wechselkosten.

Den moderierenden Einfluss des Involvements auf die Wirkbeziehung zwischen Wechselkosten und Wechselabsicht beschreibt die folgende Hypothese:

\section{Hypothese 17}

Je höher das Involvement eines Konsumenten ist, desto stärker ist der Einfluss der Wechselkosten auf die Wechselabsicht.

Analog dazu widmet sich die folgende Hypothese dem moderierenden Einfluss des Involvements auf die Wirkbeziehung zwischen Wechselkosten und Wechselvollzug:

\section{Hypothese 18}

Je höher das Involvement eines Konsumenten ist, desto stärker ist der Einfluss der Wechselkosten auf den Wechselvollzug.

Die direkten Wirkungen der Attraktivität der Alternativen im Rahmen des Wechselverhaltens von Konsumenten sind Inhalt der Hypothesen $\mathrm{H}_{3}$ und $\mathrm{H}_{4}$. Auch sie werden möglicherweise durch das Involvement moderiert. So merken BloEmer ET AL. (1996, S. 5) an, dass,$[. .$.$] we might expect an evaluation made under high involvement conditions to be$ more well-considered than an evaluation made under low involvement conditions. This implies that the effect of a high involvement evaluation will be more pronounced than the effect of a low involvement evaluation." 
Den moderierenden Einfluss des Involvements auf die Wirkbeziehung zwischen der Attraktivität der Alternativen und der Wechselabsicht beschreibt die folgende Hypothese:

\section{Hypothese 19}

Je höher das Involvement eines Konsumenten ist, desto stärker ist der Einfluss der Attraktivität der Alternativen auf die Wechselabsicht.

Analog dazu widmet sich die folgende Hypothese dem moderierenden Einfluss des Involvements auf die Wirkbeziehung zwischen der Attraktivität der Alternativen und dem Wechselvollzug:

\section{Hypothese 20}

Je höher das Involvement eines Konsumenten ist, desto stärker ist der Einfluss der Attraktivität der Alternativen auf den Wechselvollzug.

\subsection{Modell des Wechselverhaltens}

Die in den vorangegangenen Abschnitten entwickelten Hypothesen lassen sich zu einem Hypothesensystem zusammenfügen. Betrachtet man allein die Hypothesen über die direkten Wirkbeziehungen, ergibt sich das in Abb. 4.5 gezeigte Modell. Die Pfeile dieses Modells stehen für die Hypothesen zu den direkten Wirkbeziehungen: Zum einen werden Wirkungen von der Kundenzufriedenheit, der Attraktivität der Alternativen, den Wechselkosten und dem Involvement auf die Wechselabsicht vermutet, zum anderen wirken diese Größen vermutlich auf den Wechselvollzug.

Die Wirkweise der Hypothesen ist jeweils mit plus oder minus gekennzeichnet: Eine Erhöhung der unabhängigen Variable sollte bei einer mit plus ausgezeichneten Hypothese zu einer Erhöhung der abhängigen Variable und bei einer mit minus versehenen Hypothese zu einer Verringerung der abhängigen Variable führen.

Als Besonderheit fällt auf, dass die beiden Konstrukte Kundenzufriedenheit und Attraktivität der Alternativen im Modell dupliziert sind. Es handelt sich hierbei um einen Vorgriff auf die Operationalisierung (s. Abschnitt 6.3): Für Kunden, die ihren Anbieter gewechselt haben, beziehen sich die Kundenzufriedenheit und die Attraktivität der Alternativen bei der Betrachtung der aktuellen Wechselabsicht auf einen anderen Anbieter als bei den Kunden, die ihren Anbieter nicht gewechselt haben. Da sich das bewertete Objekt (nämlich der Anbieter bzw. die Alternativen) unterscheidet, müssen zwei separate Konstrukte analysiert werden (RossiteR, 2002).

Das einfache Modell der direkten Effekte aus Abb. 4.5 erfährt eine Erweiterung durch die moderierenden Effekte. Das so entstandene Modell als graphische Repräsentation des 


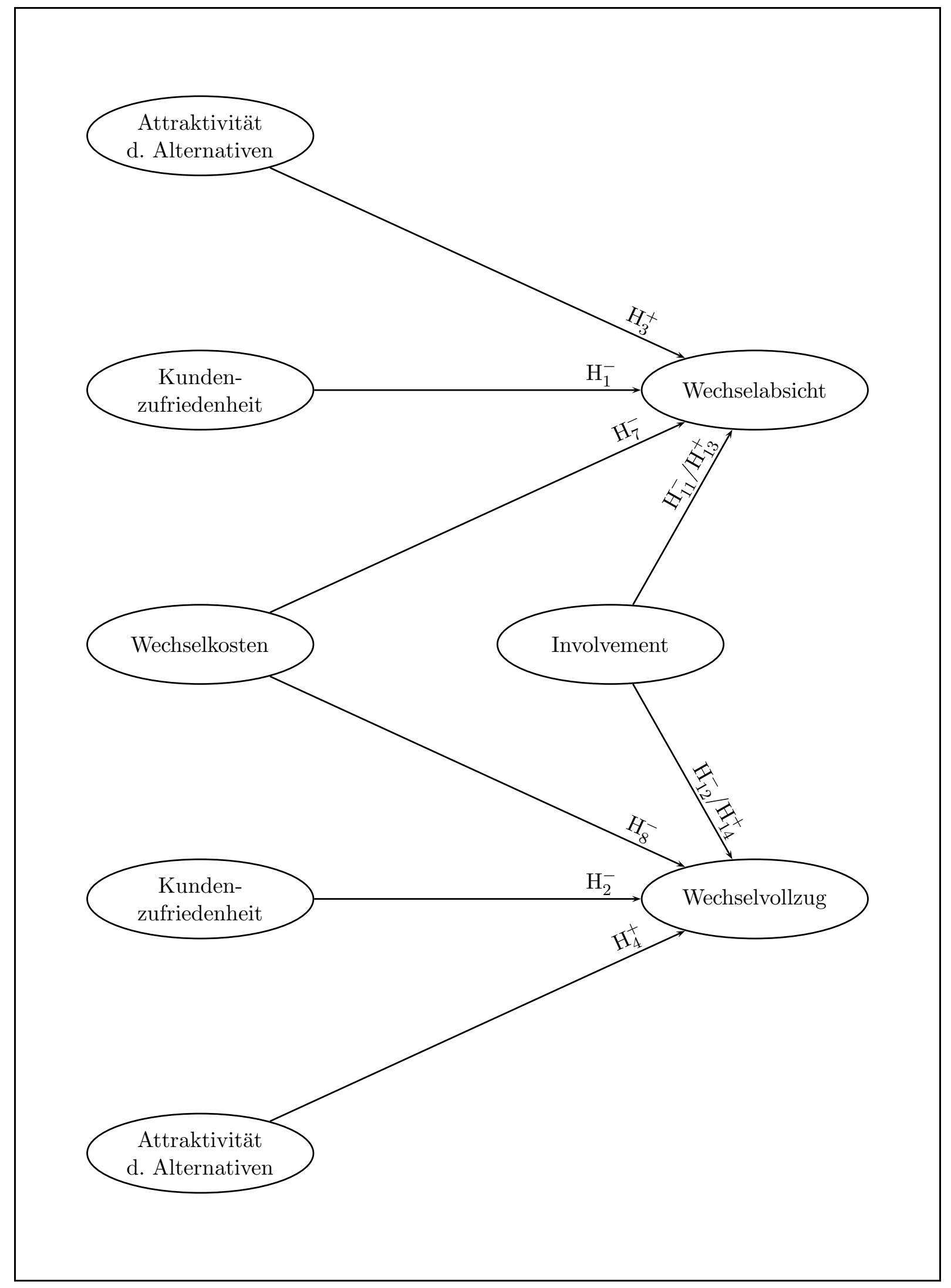

Abbildung 4.5: Hypothesensystem der direkten Wirkbeziehungen beim Wechselverhalten von Konsumenten im Markt für Haushaltsstrom 
in diesem Kapitel entwickelten Hypothesensystems zeigt Abb. 4.6. Die Pfeile, die moderierende Effekte darstellen, sind gepunktet eingezeichnet. Eine empirische Überprüfung dieses Modells ist der Inhalt des Kapitels 6.

Die Vorgehensweise, sowohl die Verhaltensabsicht als auch das bereits realisierte Verhalten zu analysieren, lehnt sich an die Untersuchung von Doney und CAnnon (1997) an. Ein wesentlicher Unterschied zwischen ihrer und der vorliegenden Untersuchung liegt jedoch in der Wahl der statistischen Methoden. Während Doney und CAnnon einen Zwei-Phasen-Ansatz wählen, indem sie zunächst eine schrittweise lineare Regression und anschließend eine logistische Regression durchführen, sollen in dieser Arbeit mittels der Partial-Least-Squares-Pfadmodellierung simultan die dichotome Variable „Wechselvollzug“ und die latente Variable „Wechselabsicht“ innerhalb eines Gesamtsystems modelliert werden. Eine Einführung in die Strukturgleichungsmodellierung mit PLS-Pfadmodellen und die Funktionsweise des PLS-Algorithmus gibt das nachfolgende Kapitel 5. 


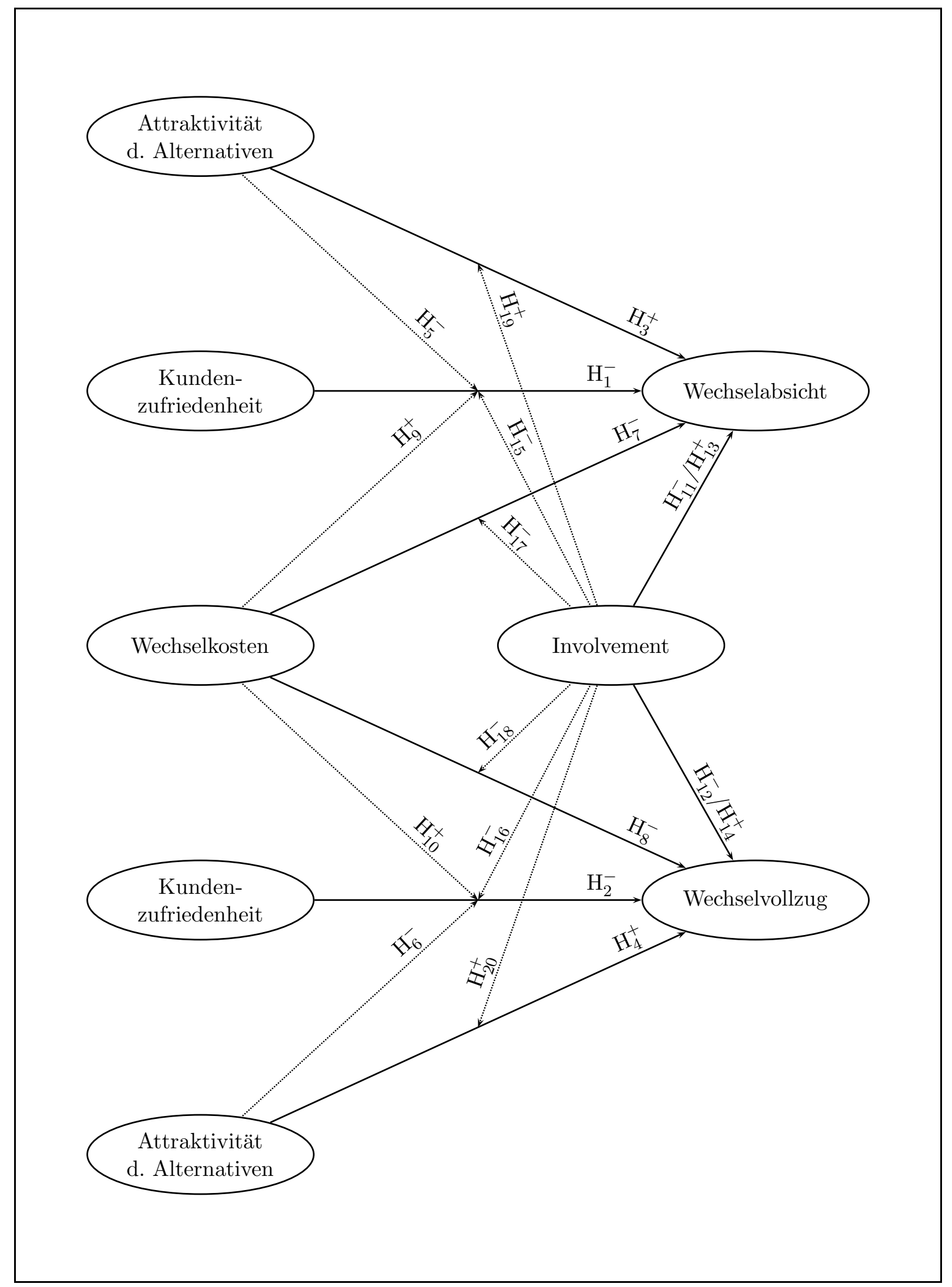

Abbildung 4.6: Hypothesensystem der direkten und moderierenden Effekte beim Wechselverhalten von Konsumenten im Markt für Haushaltsstrom 


\section{Strukturgleichungsmodellierung mit latenten Variablen}

Theoretische Konstrukte wie bspw. Kundenzufriedenheit oder Involvement können als abstrakte Gebilde, die den „wahren“, nicht beobachtbaren Zustand bzw. die Natur eines Phänomens repräsentieren, verstanden werden (BAGOzZI und FornelL, 1982, S. 24). Da es sich bei Konstrukten um nicht direkt messbare Größen handelt, bezeichnet man sie auch als latente Variablen. Die Messung von Konstrukten bedarf der Spezifikation von Beziehungen zwischen beobachtbaren Variablen - synonyme Begriffe dafür sind auch manifeste Variablen, Indikatorvariablen oder kurz Indikatoren - und dem relevanten Konstrukt. Durch diese Zusammenhänge werden Konstrukte empirisch greifbar, also messbar. In der Regel erfasst man das Konstrukt über mehrere Indikatoren, da die Beschreibung des relevanten Phänomens über einen einzelnen Indikator meist zu unbefriedigenden Ergebnissen führt (Homburg und Giering, 1996, S. 6).

Um Theorien in den Wirtschafts- und Sozialwissenschaften empirisch überprüfen zu können, benötigen Forscher geeignete Methoden zur Modellierung und statistischen Analyse. Diese Methoden müssen einigen Mindestanforderungen genügen (BARCLAY ET AL., 1995, S. 287). Zum einen sollten sie berücksichtigen, dass in Theoriesystemen die Bedeutung von Konstrukten von drei Seiten bestimmt wird: der Definition, dem spezifischen theoretischen Kontext, in den die Konstrukte eingebettet sind, und der konkreten Messung der Konstrukte. Zum anderen sollten diese Methoden sich an den Stand der Entwicklung einer vorgegebenen Theorie anpassen lassen, mit Theoriesystemen bzw. nomologischen Netzen von Konstrukten arbeiten können, Fehler im Messmodell erkennen und behandeln können sowie in der Theorie begründete Fehler wie bspw. eine erweiterte Bedeutung in Konstrukten und unerklärte Varianz entdecken können.

Die in der Psychometrie entwickelte Strukturgleichungsmodellierung mit latenten Variablen erfüllt diese Anforderungen, indem sie simultan einerseits die Qualität der Konstruktmessung hinsichtlich der Reliabilität und Validität untersucht und andererseits die Stärke der Beziehungen zwischen den Konstrukten schätzt. Bei der Strukturgleichungsmodellierung mit latenten Variablen haben sich zwei Verfahren etabliert, die den oben genannten Anforderungen gerecht werden: auf der einen Seite die sog. Kausalanalyse (oder in exakter Formulierung: Kovarianzstrukturanalyse, vgl. HomBURG, 1992, S. 500), 
die maßgeblich von Jöreskog und SörBom (siehe z. B. 1979) vorangebracht und als LISREL implementiert wurde; auf der anderen Seite die von WoLD entwickelte PartialLeast-Squares-Pfadmodellierung (PLS). ForNELL (1982) zählt beide Verfahren zu einer „zweiten Generation multivariater Analysemethoden“. Zwar positionieren JöREsKoG und Wold (1982) ihre Verfahren leicht unterschiedlich: LISREL für die Entwicklung und Überprüfung von Theorien, PLS-Pfadmodellierung eher für Prognoseanwendungen. Diese Einteilung steht auch in Zusammenhang mit der Empfehlung von WoLD (1980, S. 51), die PLS-Pfadmodellierung insbesondere dann einzusetzen, wenn die zu untersuchenden Probleme komplex und durch Theorien erst schwach durchdrungen sind. Diese Unterteilung nach dem Untersuchungszweck ist jedoch eher willkürlich. Neuere Arbeiten wie z. B. die von Hulland (1999) zeigen, dass sich die PLS-Pfadmodellierung sehr gut zur konfirmatorischen Arbeit, also zum Überprüfen von Theorien, eignet.

Die Verwendung von Strukturgleichungsmodellen hat sich zu einem Quasi-Standard in Teilen der Marketingwissenschaft entwickelt (Homburg und Baumgartner, 1998, S. 345). Ihre große Verbreitung hat JARVIS ET AL. (2003, S. 207) schon zu der verwunderten Frage verleitet, warum in der angesehenen Zeitschrift „Marketing Science“ nur sehr wenige Strukturgleichungsmodelle veröffentlicht wurden.

Hauptsächlich die folgenden beiden Gründe sprechen dafür, im Rahmen dieser Arbeit die PLS-Pfadmodellierung einzusetzen: Zum einen vermag die PLS-Pfadmodellierung im Gegensatz zur Kovarianzstrukturanalyse auch ohne Einschränkungen formative Konstrukte zu schätzen (Götz und Lienr-GobBers, 2004, S. 1). Zum anderen erfordert die PLS-Pfadanalyse nicht die Annahme der Multinormalverteilung der manifesten Variablen (Chin, 1998b, S. 295), sodass sich auch extrem schief verteilte Variablen wie z. B. Kundenzufriedenheit (Fornell, 1995, S. G205) oder dichotome Variablen wie bspw. der Wechselvollzug in die Analyse mit einschließen lassen.

Die folgenden Unterabschnitte beschreiben zunächst die Entwicklung und den Aufbau von PLS-Pfadmodellen, bevor dann der eigentliche Schätzalgorithmus vorgestellt wird. Abschließend wird auf besondere Eigenschaften von PLS-Pfadmodellen eingegangen.

\subsection{Aufbau von Strukturgleichungsmodellen}

Strukturgleichungsmodelle mit latenten Variablen - also auch PLS-Pfadmodelle - bestehen typischerweise aus zwei Submodellen: dem Strukturmodell, das die Beziehungen zwischen den latenten Variablen nachbildet, und dem Messmodell, das die Beziehungen zwischen den latenten Variablen und den manifesten Variablen festlegt. Abb. 5.1 veranschaulicht das Zusammenwirken beider Submodelle innerhalb eines Pfadmodells. Die 


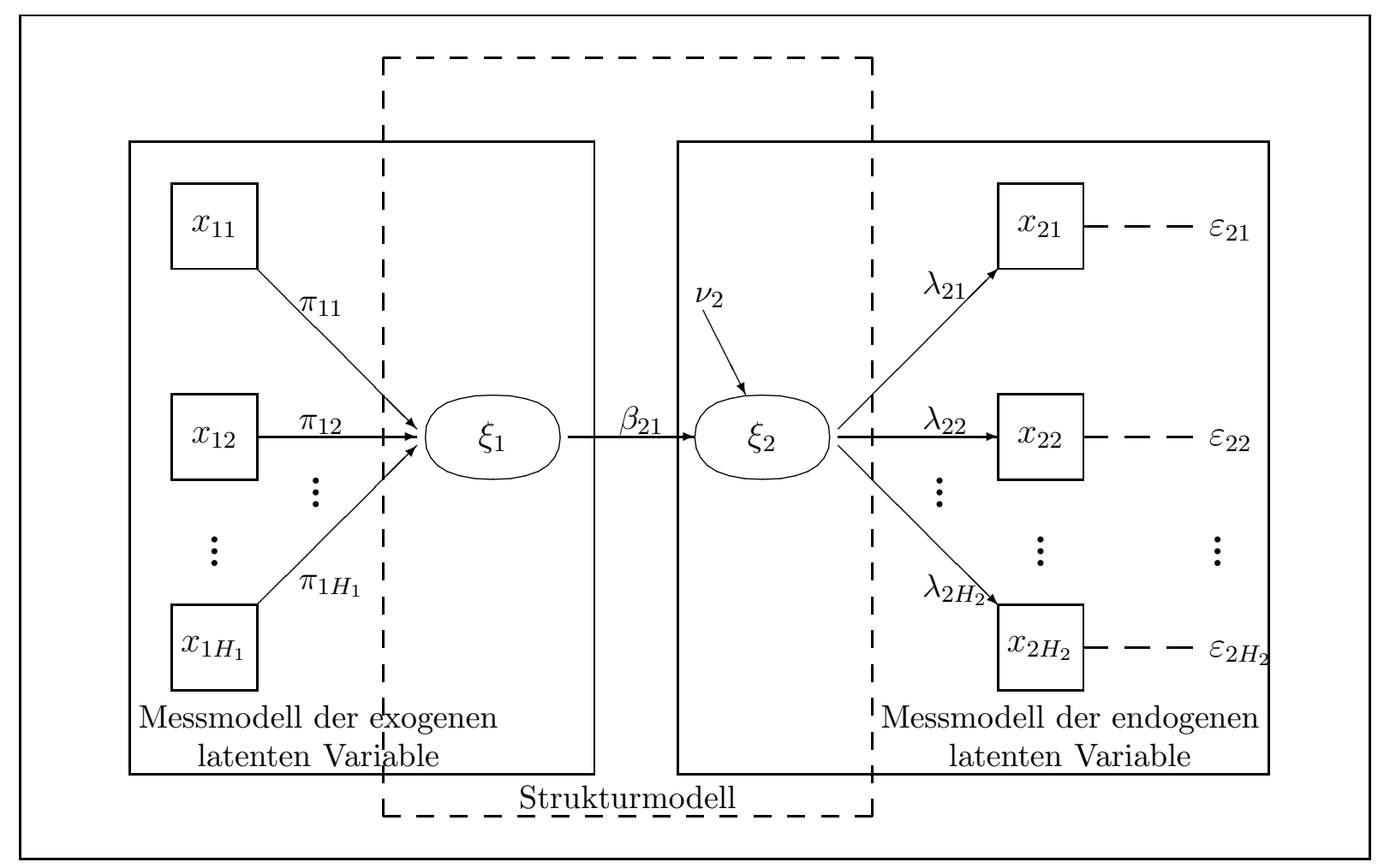

Abbildung 5.1: Modell mit zwei latenten Variablen

Spezifikation der beiden Submodelle bildet die Grundlage eines PLS-Pfadmodells; man spricht in diesem Zusammenhang auch von Prädiktorspezifikation. Die Prädiktorspezifikation ist eine wesentliche, durch den Forscher zu erbringende Vorleistung.

\subsubsection{Strukturmodell}

Das Strukturmodell (oder innere Modell) beschreibt die Beziehungen zwischen den einzelnen latenten Variablen. Im Strukturmodell lässt sich jede latente Variable $\xi_{j}$ in Abhängigkeit der übrigen latenten Variablen $\xi_{i}$ darstellen:

$$
\xi_{j}=\beta_{j 0}+\sum_{i \neq j} \beta_{j i} \xi_{i}+\nu_{j}
$$

Eine latente Variable, die von keiner anderen latenten Variablen abhängt, wird als exogen bezeichnet. Anderenfalls spricht man von einer endogenen latenten Variable. Bei der PLS-Pfadmodellierung ist eine wichtige Anforderung an ein gültiges Strukturmodell die sog. Rekursivität: Innerhalb des Strukturmodells darf es keine kausale Schleife geben, d.h. es darf von keiner latenten Variablen eine Kette von Pfeilen ausgehen, die direkt oder indirekt über andere latente Variablen wieder auf die ursprüngliche latente Variable 
zeigt. LOHMÖLLER (1989) entwickelte mit der sog. inneren Designmatrix eine zusammenfassende Darstellung des Strukturmodells. Die innere Designmatrix $D$ ist quadratisch und besitzt nur binäre Elemente. Die Anzahl der Zeilen (also auch der Spalten) entspricht der Anzahl der latenten Variablen des Modells. Ein Element $d_{i j}$ der inneren Designmatrix ist gleich 1, wenn eine Wirkbeziehung von der latenten Variable $\xi_{i}$ auf die latente Variable $\xi_{j}$ besteht; ansonsten ist es 0. Sofern das Strukturmodell rekursiv ist, lässt sich die innere Designmatrix als eine untere Dreiecksmatrix darstellen, in der alle Elemente der Hauptdiagonale gleich 0 sind.

Die Spezifikation des Strukturmodells liegt in den Händen des Forschers. Hierbei sollte er solche latente Variablen mit Pfeilen verbinden, zwischen denen er - ausgehend z. B. von bestehenden Theorien oder qualitativen Voruntersuchungen - einen Wirkzusammenhang vermutet.

\subsubsection{Messmodell}

Das Messmodell (oder äußere Modell) gibt an, wie die latenten Variablen $\xi_{j}$ mit den manifesten Variablen $x_{j h}$ in Verbindung gebracht werden können. Bei der Spezifikation des Messmodells müssen zum Einen den latenten Variablen manifeste Variablen zugeordnet werden, die die latenten Variablen möglichst gut messen. Zum Anderen ist die Form des Messmodells festzulegen. Zwei Formen von Messmodellen haben sich etabliert: reflektive und formative. Eine ausführliche Diskussion zum Einsatz formativer und reflektiver Messmodelle findet sich bei EgGert und Fassott (2003).

Bei der Verwendung des reflektiven Messmodells unterstellt man, dass die Ausprägung einer latenten Variable die Höhe der zugehörigen manifesten Variablen verursacht. Die Pfeile in der graphischen Darstellung 5.2 symbolisieren die unterstellte Wirkrichtung, indem sie ausgehend von der latenten Variable auf die manifesten Variablen zeigen.

In reflektiven Messmodellen ist jede manifeste Variable mit ihrer latenten Variable mittels einfacher Regression verbunden:

$$
x_{j h}=\lambda_{j h 0}+\lambda_{j h} \xi_{j}+\varepsilon_{j h}
$$

Reflektiv bedeutet also, dass jede manifeste Variable $x_{j h}$ ihre latente Variable $\xi_{j}$ reflektiert. Zugleich unterstellt ein reflektives Messmodell, dass eine Änderung der latenten Variablen immer auch mit einer Änderung aller zugehörigen manifesten Variablen einhergeht. Die Ladungen $\lambda_{j h}$ sorgen hierbei für die Verbindung zwischen den manifesten Variablen $x_{j h}$ und den latenten Variablen $\xi_{j}$. 


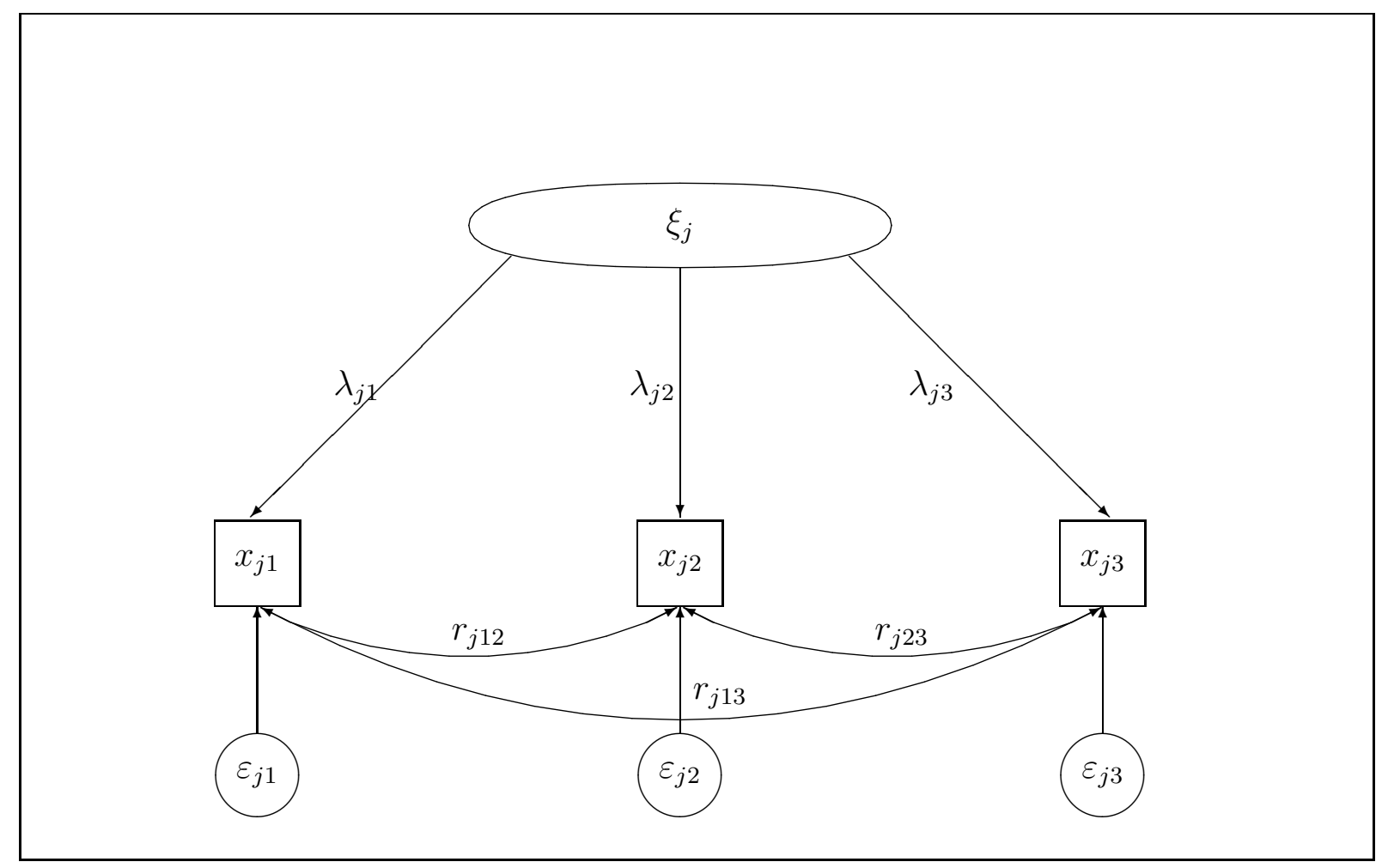

Abbildung 5.2: Reflektives Messmodell der latenten Variable $\xi_{j}$ mit drei manifesten Variablen $x_{j h}$ und zugehörigen Messfehlern $\varepsilon_{j h}$ sowie den zugehörigen Ladungen $\lambda_{j h}$ und Korrelationen $r_{j g h}$

Das formative Messmodell spiegelt einen umgekehrten Wirkzusammenhang wider: Hier verursachen die Werte der manifesten Variablen die Ausprägung der zugeordneten latenten Variable. Die Richtung der Pfeile in Abb. 5.3 verdeutlicht dies: Sie zeigen von den manifesten Variablen zur latenten Variable.

Ändert sich einer der Indikatoren einer formativ modellierten latenten Variable, so verändert sich notwendigerweise auch der Wert der latenten Variable selbst. Mathematisch betrachtet ist die latente Variable $\xi_{j}$ eine Linearkombination ihrer manifesten Variablen $x_{j h}$ (BOLlen und LenNox, 1991, S. 306):

$$
\xi_{j}=\sum_{h} \pi_{j h} x_{j h}+\delta_{j}
$$

Die Koeffizienten $\pi_{j h}$ geben dabei die Gewichtung der Indikatoren $x_{j h}$ bei ihrer linearkombinatorischen Verrechnung zu der latenten Variable $\xi_{j}$ an und werden daher als Gewichte bezeichnet. Wird das Messmodell als fehlerbehaftet angesehen, so bezeichnet $\delta_{j}$ den Fehlerterm der Messung (EgGert und Fassott, 2003, S. 2). PLS nimmt an, dass der Fehlerterm $\delta_{j}$ gleich null ist. 


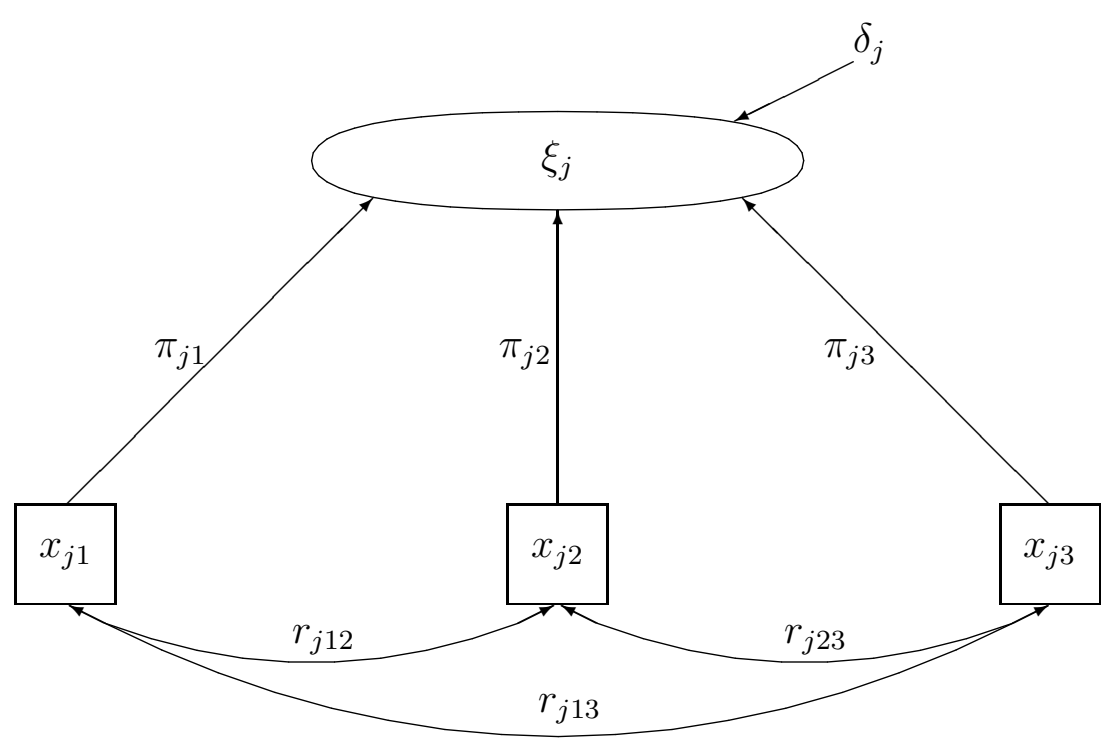

Abbildung 5.3: Formatives Messmodell der latenten Variable $\xi_{j}$ mit drei manifesten Variablen $x_{j h}$ sowie den zugehörigen Gewichten $\pi_{j h}$ und den Korrelationen $r_{g h}$

Anders als bei reflektiven Messmodellen muss bei formativen Messmodellen eine Änderung der latenten Variablen nicht zwingend mit einer Änderung aller zugehörigen manifesten Variablen verbunden sein. Vielmehr kann eine Änderung einer latenten Variablen lediglich auf der Änderung von nur einer manifesten Variablen beruhen.

Der Unterschied zwischen den beiden Formen von Messmodellen lässt sich sehr gut am Beispiel des Konstrukts „Trunkenheit“ demonstrieren (vgl. Abb. 5.4). Trunkenheit lässt sich mit formativen, aber auch mit reflektiven Indikatoren messen. Zu einer Messung mit reflektiven Indikatoren sollten sinnvollerweise die Folgen von Trunkenheit herangezogen werden. Mögliche Indikatoren wären bspw. Tests, ob ein Proband beim Pusten nach Alkohol riecht, ob er noch auf gerader Linie gehen kann oder ob er ein Geldstück zu fangen vermag. Formative Indikatoren sind angebracht, will man die Ursachen von Trunkenheit erfassen. Hierzu sollten auf jeden Fall die konsumierten Mengen an Bier, Wein und Spirituosen gemessen werden.

JARVIS ET AL. (2003, S. 203) präsentieren ein Regelwerk, anhand dessen sich aus gegebenen Indikatoren ex post ermitteln lässt, ob ein Konstrukt formativ oder reflektiv ist. Dieses Regelwerk setzt sich aus vier Fragenkomplexen zusammen: der Kausalitätsrichtung 


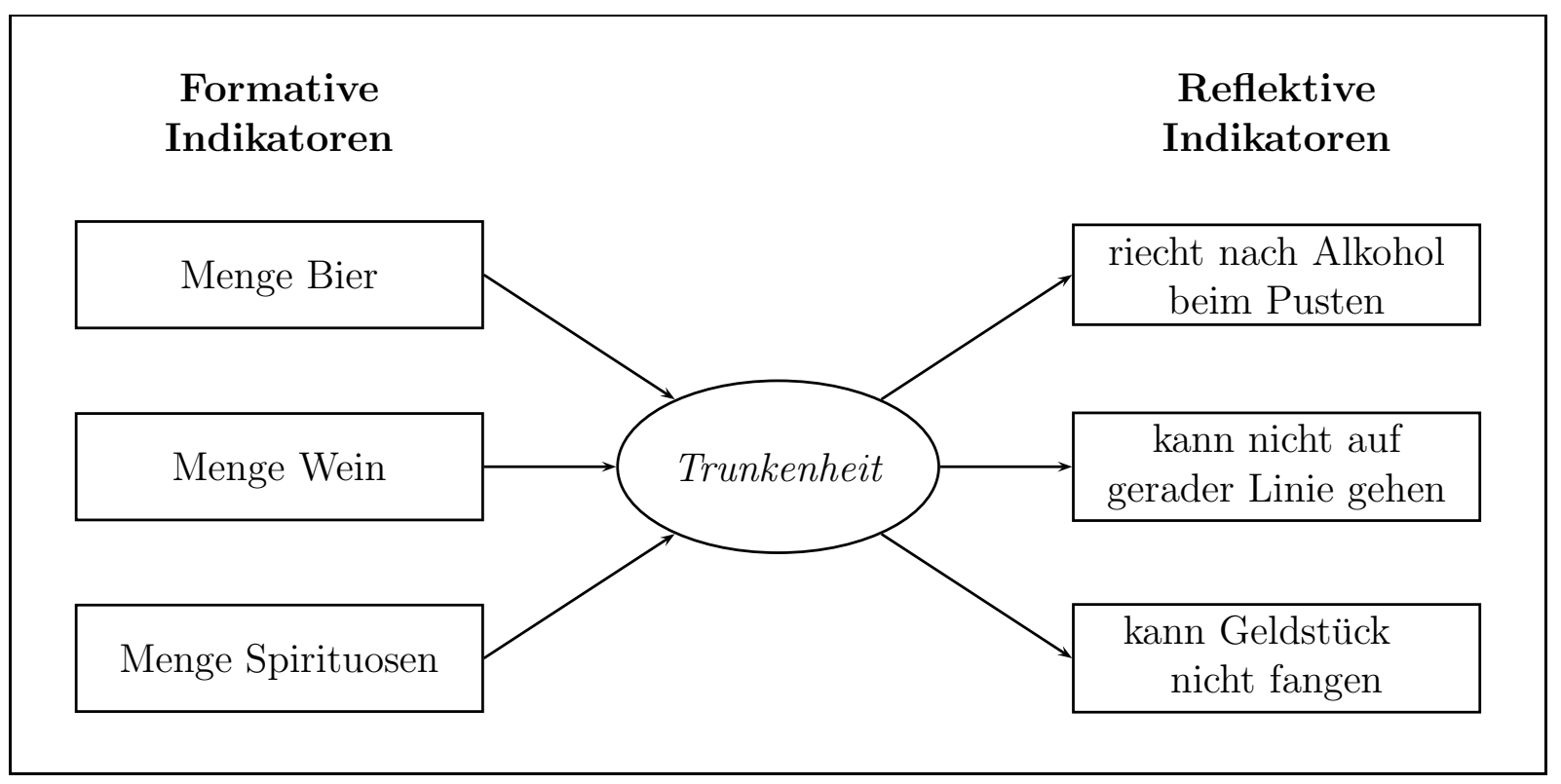

Abbildung 5.4: Formative und reflektive Indikatoren am Beispiel des Konstrukts „Trunkenheit" (in Anlehnung an AlBers, 2004)

zwischen dem Konstrukt und seinen Indikatoren, der Austauschbarkeit der Indikatoren, der gemeinsamen Kovarianz der Indikatoren sowie dem nomologischen Netz der Konstruktindikatoren.

1. Der erste Fragenkomplex bezieht sich auf die Kausalitätsrichtung zwischen dem Konstrukt und seinen Indikatoren.

a) In welche Richtung orientiert sich die Kausalität zwischen dem Konstrukt und dem Messmodell? Bei formativen Messmodellen geht die Ursache-WirkungsBeziehung von den Indikatoren zum Konstrukt, bei reflektiven vom Konstrukt zu den Indikatoren.

b) Sind die Indikatoren definierende Charakteristika oder Erscheinungsformen des Konstrukts? Bei formativen Messmodellen definieren die Indikatoren das Konstrukt, bei reflektiven sind die Indikatoren Erscheinungsformen des Konstrukts.

c) Führen Indikatoränderungen zu Konstruktänderungen oder nicht? Bei formativen Messmodellen sollten Indikatoränderungen zu Konstruktänderungen führen, bei reflektiven nicht.

d) Führen Konstruktänderungen zu Indikatoränderungen oder nicht? Bei formativen Messmodellen sollten Konstruktänderungen keine Auswirkungen auf die Indikatoren haben, wohingegen bei reflektiven Messmodellen Konstruktänderungen Indikatoränderungen bewirken sollten. 
2. Der zweite Fragenkomplex bezieht sich auf die Austauschbarkeit von Indikatoren.

a) Inwiefern müssen die Indikatoren austauschbar sein? Bei formativen Messmodellen müssen die Indikatoren nicht austauschbar sein, bei reflektiven Messmodellen sollten sie jedoch austauschbar sein.

b) Sollten die Indikatoren denselben oder einen ähnlichen Inhalt besitzen bzw. sollten sie ein gemeinsames Thema haben? Bei formativen Messmodellen müssen die Indikatoren nicht denselben oder einen ähnlichen Inhalt besitzen bzw. müssen kein gemeinsames Thema haben, bei reflektiven sollten die Indikatoren hingegen denselben oder einen ähnlichen Inhalt besitzen bzw. ein gemeinsames Thema haben.

c) Inwieweit verändert sich der Begriffsbereich des Konstrukts, wenn ein Indikator wegfällt? Bei formativen Messmodellen kann der Wegfall eines Indikators den Begriffsbereich eines Konstrukts verändern, bei reflektiven sollte sich der Begriffsbereich eines Konstrukts nicht verändern.

3. Der dritte Fragenkomplex bezieht sich auf die Kovarianz zwischen den Indikatoren.

a) Inwieweit müssen die Indikatoren untereinander kovariieren? Bei formativen Messmodellen müssen die Indikatoren nicht zwingend kovariieren, bei reflektiven ist Kovarianz zwischen den Indikatoren eine notwendige Bedingung.

b) Sollten Änderungen eines Indikators einhergehen mit Änderungen der anderen Indikatoren? Bei formativen Messmodellen müssen Änderungen eines Indikators nicht mit Änderungen der anderen Indikatoren einhergehen, anders bei reflektiven.

4. Der vierte Fragenkomplex bezieht sich auf das nomologische Netz der Indikatoren.

a) Inwieweit befinden sich die Indikatoren im gleichen nomologischen Netz? Bei formativen Messmodellen kann das nomologische Netz der Indikatoren unterschiedlich sein, bei reflektiven sollten die Indikatoren in das gleiche nomologische Netz eingebettet sein.

b) Sollten die Indikatoren jeweils die gleichen Ursachen und Konsequenzen haben? Bei formativen Messmodellen brauchen die Indikatoren nicht die gleichen Ursachen und Konsequenzen zu haben, während dies für die Indikatoren reflektiver Messmodelle notwendig ist.

Ergeben sich bei der Überprüfung eines Konstrukts Widersprüche, d. h. es sprechen Kriterien sowohl für eine formative als auch für eine reflektive Operationalisierung, empfehlen JARVIS ET AL. (2003, S. 203), die Konstruktkonzeptualisierung zu verfeinern. 


\subsubsection{Modellierung von Konstrukten zweiter Ordnung}

Oftmals umfasst ein Konstrukt einen sehr komplexen Sachverhalt. Eine einfache Operationalisierung wird dessen Charakter dann nicht gerecht, weil dadurch wichtige Facetten des Konstrukts außer Acht gelassen würden. Eine Lösung dieses Problems liegt darin, latenten Variablen eine hierarchische Struktur zu geben. Die hierachische Struktur von latenten Variablen ist Thema ausgiebiger Literatur zu Ein-Block-/Mehr-Faktor-Modellen der Psychometrie (vgl. bspw. Harman, 1976; Schmid und Leiman, 1957). Konstrukte erhalten eine hierarchische Struktur durch die Unterteilung in Konstrukte erster und zweiter (ggf. auch höherer) Ordnung. Zunächst werden auf einer unteren Ebene die einzelnen Dimensionen eines komplexen Konstrukts in Form von Komponenten, die im Prinzip eigenständige Konstrukte (sog. Konstrukte erster Ordnung) darstellen, einzeln erfasst. Anschließend werden die Konstrukte erster Ordnung auf einer zweiten Ebene zum Gesamtkonstrukt (sog. Konstrukt zweiter Ordnung) verdichtet. Die hierarchische Struktur von latenten Variablen eignet sich besonders für inhaltlich breite Konstrukte, d. h. Konstrukte, die aus mehreren Facetten oder Dimensionen bestehen.

Häufig hat der Forscher die Wahl, ein Konstrukt als eindimensional (Konstrukt erster Ordnung) oder mehrdimensional (zweiter oder höherer Ordnung) zu spezifizieren. Konstrukte höherer Ordnung erlauben die Erfassung mehrerer Dimensionen eines Konstrukts, und der Einfluss der einzelnen Dimensionen des Konstrukts innerhalb eines Netzes von Wirkzusammenhängen kann aufgezeigt werden. Für eindimensionalen Konstrukten spricht die effizientere Datenerhebung, denn gewöhnlich werden weniger Indikatoren benötigt.

Konstrukte zweiter Ordnung lassen sich durch einfach verschachtelte Messmodelle operationalisieren: Die manifesten Variablen dienen zur Bildung der Komponenten (Konstrukte erster Ordnung), die wiederum zur Erklärung des Konstrukts zweiter Ordnung dienen. Sowohl auf der Ebene zwischen manifesten Variablen und Konstrukten erster Ordnung als auch auf der Ebene zwischen den Konstrukten erster Ordnung und dem Konstrukt zweiter Ordnung ist festzulegen, ob eine formative oder eine reflektive Spezifikation gewählt werden soll. Wie Abb. 5.5 aufzeigt, existieren vier mögliche Spezifikationen:

Typ I: Sowohl die einzelnen Komponenten als auch das Gesamtkonstrukt sind reflektiv.

Typ II: Die einzelnen Komponenten sind formativ, während das Konstrukt zweiter Ordnung reflektiv ist.

Typ III: Die einzelnen Komponenten sind reflektiv, während das Konstrukt zweiter Ordnung sich formativ aus den Komponenten zusammensetzt.

Typ IV: Die einzelnen Komponenten und das Gesamtkonstrukt sind beide formativ. 


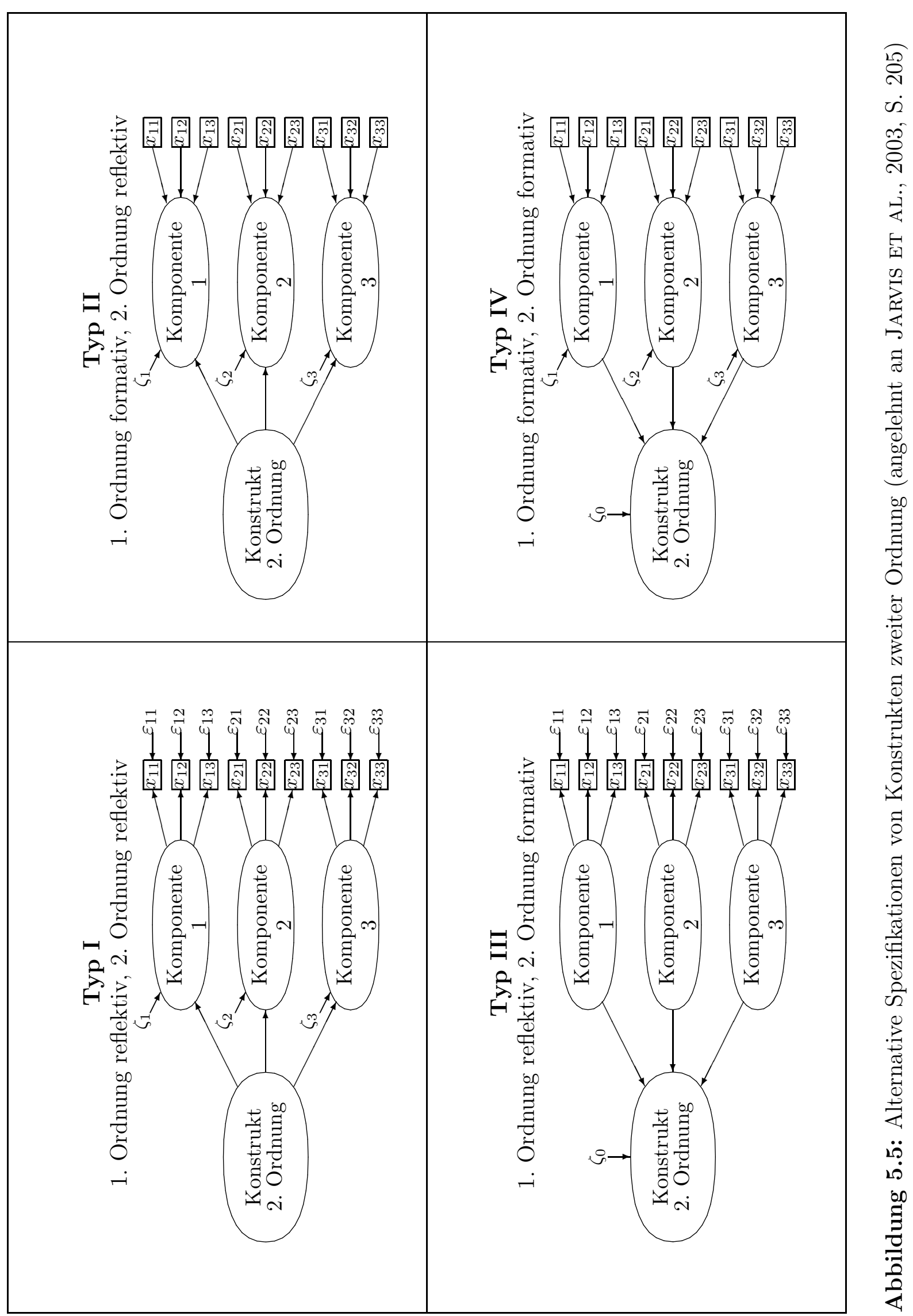




\subsubsection{Modellierung von moderierenden Effekten}

Den Ausführungen zu Strukturmodellen in Unterabschnitt 5.1.1 lag das Verständnis zu Grunde, dass zwischen einzelnen latenten Variablen direkte Zusammenhänge (sog. direkte Effekte) bestehen. Ein direkter Effekt besteht, wenn eine Veränderung der exogenen Variablen eine Veränderung der endogenen Variablen bewirkt. Neben den direkten Effekten können auch sog. Interaktions- oder moderierende Effekte auftreten. Moderierende Effekte rufen solche Variablen hervor, deren Veränderung die Richtung und/oder die Stärke der Beziehung zwischen einer exogenen und einer endogenen Variablen beeinflusst (BARON und KENNY, 1986, S. 1174).

Moderierende Effekte lassen sich auf unterschiedliche Weise in mathematische Modelle integrieren. Üblich sind die folgenden beiden Methoden:

- Eine dichotome bzw. dichotomisierte Moderatorvariable dient zur Gruppierung der Datensätze. Die so geschaffenen Datengruppen werden separat analysiert und Unterschiede in den Modellparametern zwischen den Datengruppen als Interaktionseffekte interpretiert.

- Aus der Regressions- bzw. Varianzanalyse entspringt der Ansatz, die exogene Variable und die Moderatorvariable multiplikativ zu verknüpfen und auf diese Weise den Interaktionseffekt zu quantifizieren.

Natürlich ist auch eine Kombination oder sequenzielle Abfolge beider Methoden möglich. So schlägt REINECKE (1999, S. 95) vor, zunächst multiple Gruppenvergleiche durchzuführen, um zu überprüfen, ob bedeutsame und signifikante Interaktionseffekte zu erwarten sind.

Abb. 5.6 zeigt schematisch ein einfaches Modell mit einer moderierenden latenten Variablen. Der Moderatoreffekt ist dadurch gekennzeichnet, dass der von der moderierenden Variable ausgehende Pfeil auf die Beziehung zeigt, deren Beeinflussung Inhalt der Moderatorhypothese ist.

In Abb. 5.7 ist dargestellt, wie die Prüfung moderierter Effekte nach der zweiten geschilderten Methode erfolgt. Die Moderatoren-Hypothese wird unterstützt, wenn - unabhängig von den Ausprägungen der Pfadkoeffizienten der direkten Beziehungen von Prädiktor und Moderator zur endogenen Variable - die Interaktionsbeziehung (hier Pfad c) signifikant ist, also ein signifikanter Interaktionseffekt feststellbar ist (vgl. HELM ET AL., 2005, im Druck). 
Bei der Überprüfung moderierender Effekte ist es grundsätzlich erwünscht, dass der Moderator nicht mit dem Prädiktor oder der abhängigen Variable korreliert (BARON und KenNy, 1986, S. 1174).

Chaplin (1991, S. 160-162, 168f.) zeigt auf, dass Haupteffekte den größten Anteil an der Varianzerklärung einer abhängigen Variable haben und dass selbst Regressionsmodelle mit signifikanten Interaktionstermen nur selten eine substanziell bessere Vorhersagevalidität aufweisen. „Even in the more limited domain of a specific study with a limited set of variables, interaction effects will be statistically more difficult to detect than main effects" (Chaplin, 1997, S. 880). So stellt Chaplin (1991, S. 169) fest, dass Teilkorrelationen zwischen Interaktionsterm und abhängiger Variable kaum stärker als $\pm 0,10$ sind. Er schließt daraus, dass bereits bei derart kleinen Werten die Existenz von Interaktionseffekten in Betracht zu ziehen ist.

\subsection{Der Partial-Least-Squares-Ansatz zur Modellschätzung}

\subsubsection{Entwicklung der PLS-Pfadmodellierung}

Die PLS-Pfadmodellierung wurde erstmals 1966 von WoLD unter dem Namen NILES

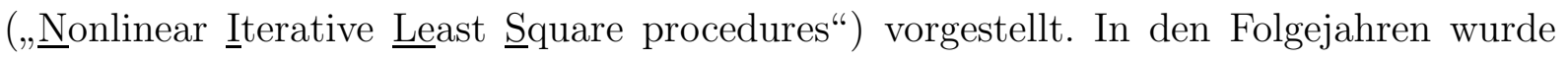
der PLS-Algorithmus insbesondere von Wold (vgl. z. B. 1969) unter der Bezeichnung

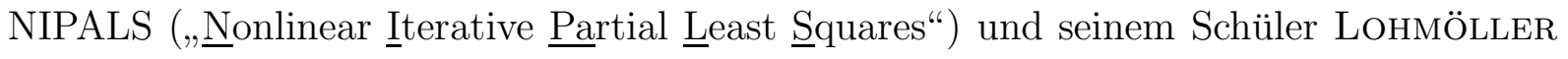
(vgl. beispielhaft 1989) unter der Bezeichnung „PLS-Pfadmodellierung“ weiterentwickelt.

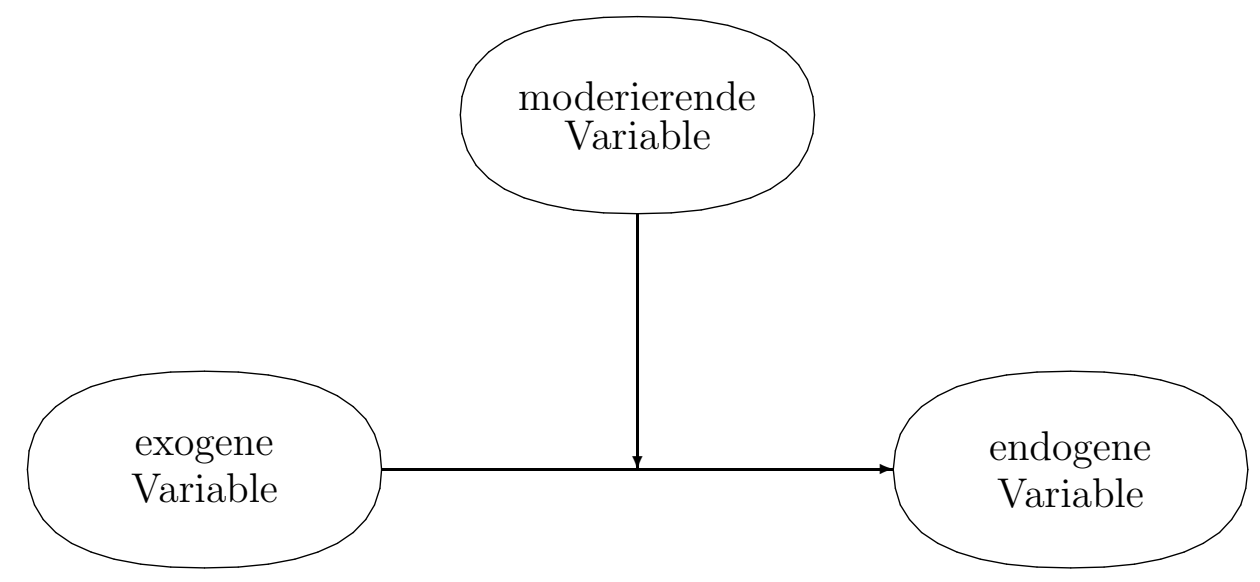

Abbildung 5.6: Beispiel für ein Modell mit moderierendem Effekt 


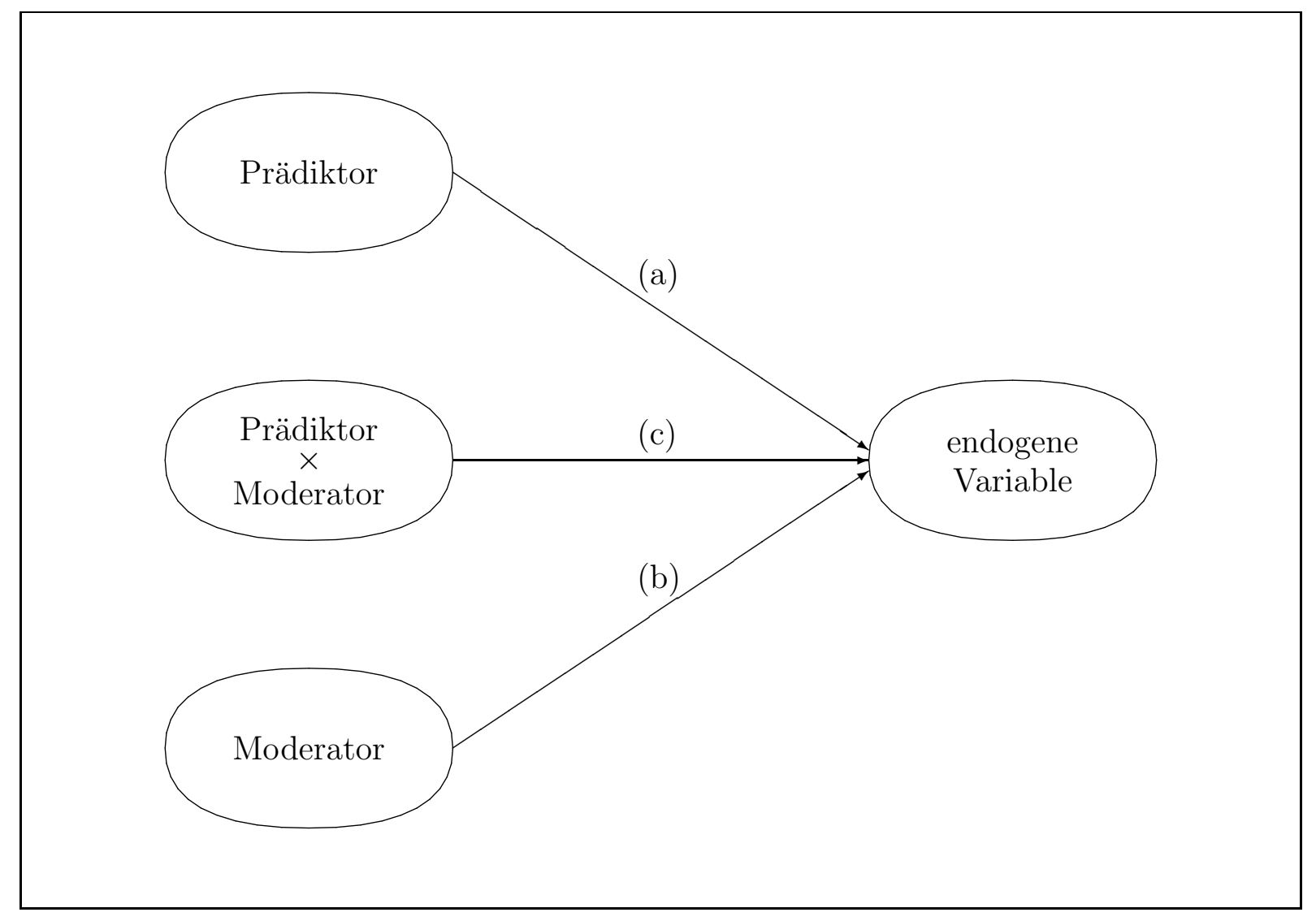

Abbildung 5.7: Transkription eines Modells mit moderierendem Effekt

Die Partial-Least-Squares-Methode (PLS-Methode) wurde von WoLD (1973) vorwiegend für die Analyse von Daten entwickelt, für die nur geringe theoretische Erklärungsansätze bestehen. WOLD bemängelte, dass LISREL in vielen Situationen nicht angewendet werden könne: In vielen Fällen sind die für die gängigen LISREL-Schätzverfahren bzw. -Modellevaluierungen erforderlichen Verteilungsannahmen unzulässig, in anderen Fällen ist die Anzahl der zur Verfügung stehenden Datensätze zu gering. Anstatt einer Analyse der Kovarianzstruktur, wie sie von LISREL durchgeführt wird, wählte WoLD eine Analyse der Datenstruktur. Dies erlaubt nicht nur die Ermittlung der Stärke der Wirkzusammenhänge, sondern auch die explizite Schätzung der Werte der latenten Variablen. Dieser Vorteil gegenüber kovarianzstrukturbasierten Verfahren kann insbesondere bei der Parameterschätzung bei Modellen mit Interaktionseffekten zum Tragen kommen.

Das Schätzverfahren setzt sich zusammen aus einer Initialisierung, dem Algorithmus zur Schätzung der latenten Variablen sowie der abschließenden Ermittlung der Strukturgleichungsparameter. Zunächst soll die Funktionsweise des Schätzverfahrens bei einem einfachen Modell mit nur zwei latenten Variablen aufgezeigt werden, bevor anschließend das Verfahren für allgemeine Modelle mit mehreren latenten Variablen erläutert wird. 


\subsubsection{Schätzung von Modellen mit zwei latenten Variablen}

Um ein Verständnis für die Funktionsweise des PLS-Algorithmus zu erhalten, eignet sich besonders die Betrachtung eines Modells mit zwei latenten Variablen. Für das Modell aus Abb. 5.1 würde das Schätzverfahren wie folgt ablaufen (BARCLAY ET AL., 1995, S. 292):

Schritt 0: Für zumindest eine latente Variable ist ein Startwert erforderlich, der nicht der Nullvektor sein darf. In der ersten PLS-Iteration erhält $\xi_{2}$ als Startwert einen beliebigen zugehörigen Indikatorwert, so z. B. $x_{21}$.

Schritt 1: Mittels einer multiplen Kleinste-Quadrate-Regression mit $\xi_{2}$ als abhängiger Variable und $x_{11}, \ldots, x_{1 H_{1}}$ als unabhängigen Variablen werden die Regressionsgewichte $\pi_{1}, \ldots, \pi_{H_{1}}$ geschätzt. Im Prinzip wird so getan, als gelte $\beta_{21}=1$.

Schritt 2: Nun lässt sich $\xi_{1}$ bilden als Linearkombination aus $x_{11}, \ldots, x_{1 H_{1}}$ mit den Gewichten $\pi_{11}, \ldots, \pi_{1 H_{1}}$, d. h. $\xi_{1}=\sum_{h=1}^{H_{1}} \pi_{1 h} \cdot x_{1 h}$.

Schritt 3: Die Ladungen $\lambda_{21}, \ldots, \lambda_{2 H_{2}}$ werden dann mittels einer Serie einfacher Regressionen mit $\xi_{1}$ als unabhängiger Variable und $x_{21}, \ldots, x_{2 H_{2}}$ als abhängigen Variablen geschätzt. Die Ladungen $\lambda_{21}, \ldots, \lambda_{2 H_{2}}$ werden in Gewichte umgeformt.

Schritt 4: Mit diesen Gewichten wird ein neuer Schätzwert für $\xi_{2}$ als Linearkombination aus $x_{21}, \ldots, x_{2 H_{2}}$ ermittelt.

Die Schritte 1 bis 4 werden so lange wiederholt, bis ein vorzugebendes Abbruchkriterium erfüllt ist. Die Beschreibung des Algorithmus lässt seine Funktionsweise leicht erkennen: Während man einen Teil des Modells als gegeben betrachtet, wird der andere Teil mittels Regressionen neu berechnet. Daher stammt auch die Bezeichnung Partial Least Squares (PLS), also „partielle kleinste Quadrate“. Während das Messmodell inklusive der Werte für $\xi_{1}$ und $\xi_{2}$ nun komplett bestimmt ist, fehlt noch die Schätzung des Strukturmodells. Den entsprechenden Schätzer $\beta_{21}$ liefert eine einfache Regression mit $\xi_{1}$ als unabhängiger und $\xi_{2}$ als abhängiger Variable. Aus dieser Regression erhält man des Weiteren $R^{2}$ als Gütemaß dafür, wie gut $\xi_{2}$ von $\xi_{1}$ erklärt wird.

Eine graphische Darstellung des Algorithmus findet sich in Abb. 5.8.

\subsubsection{Schätzung von Modellen mit mehreren latenten Variablen}

Bei einem Modell mit mehr als zwei Konstrukten, wie es bspw. Abb. 5.9 zeigt, muss der Algorithmus modifiziert werden. Aus Gründen der Übersichtlichkeit wird für die folgende 


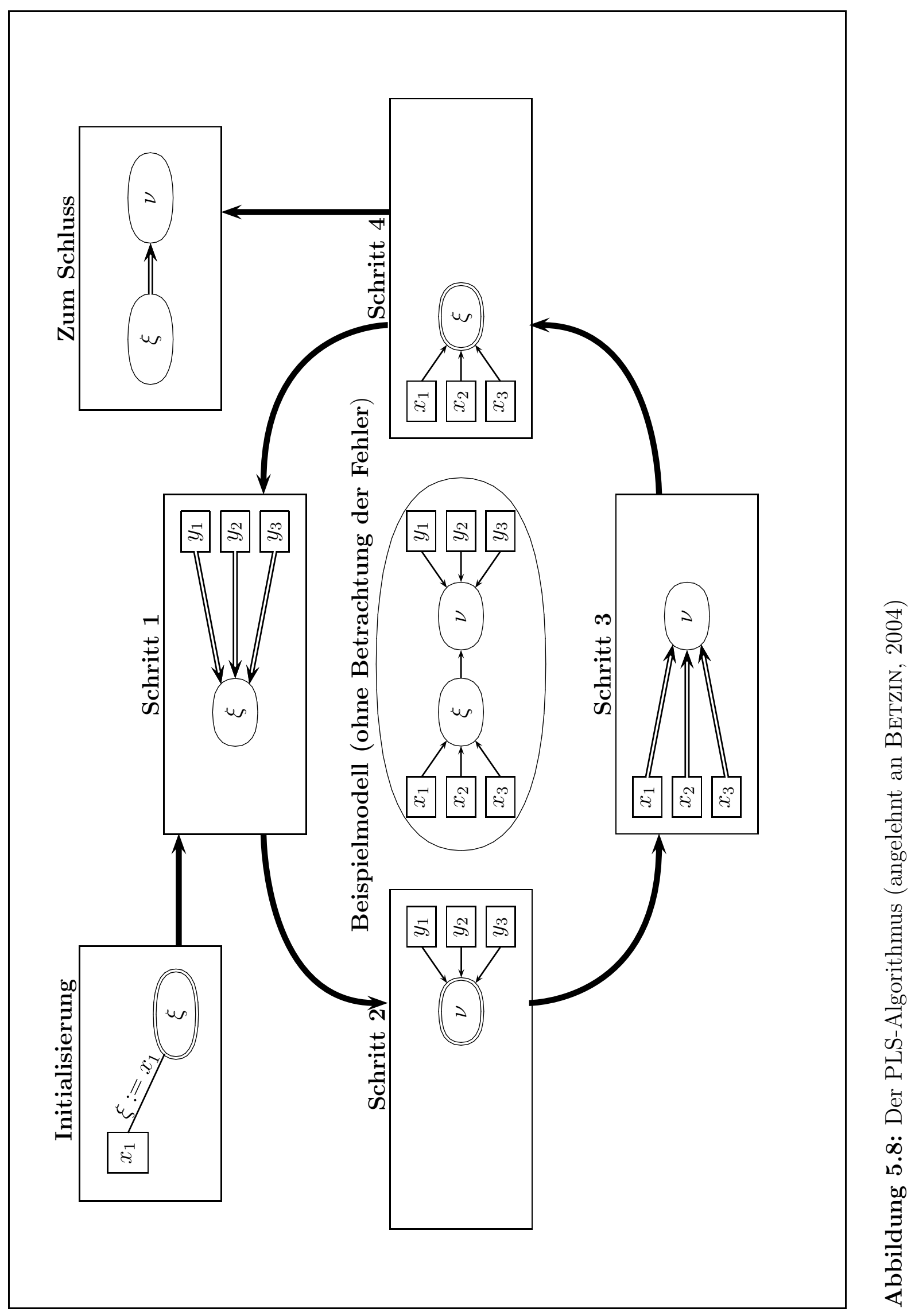




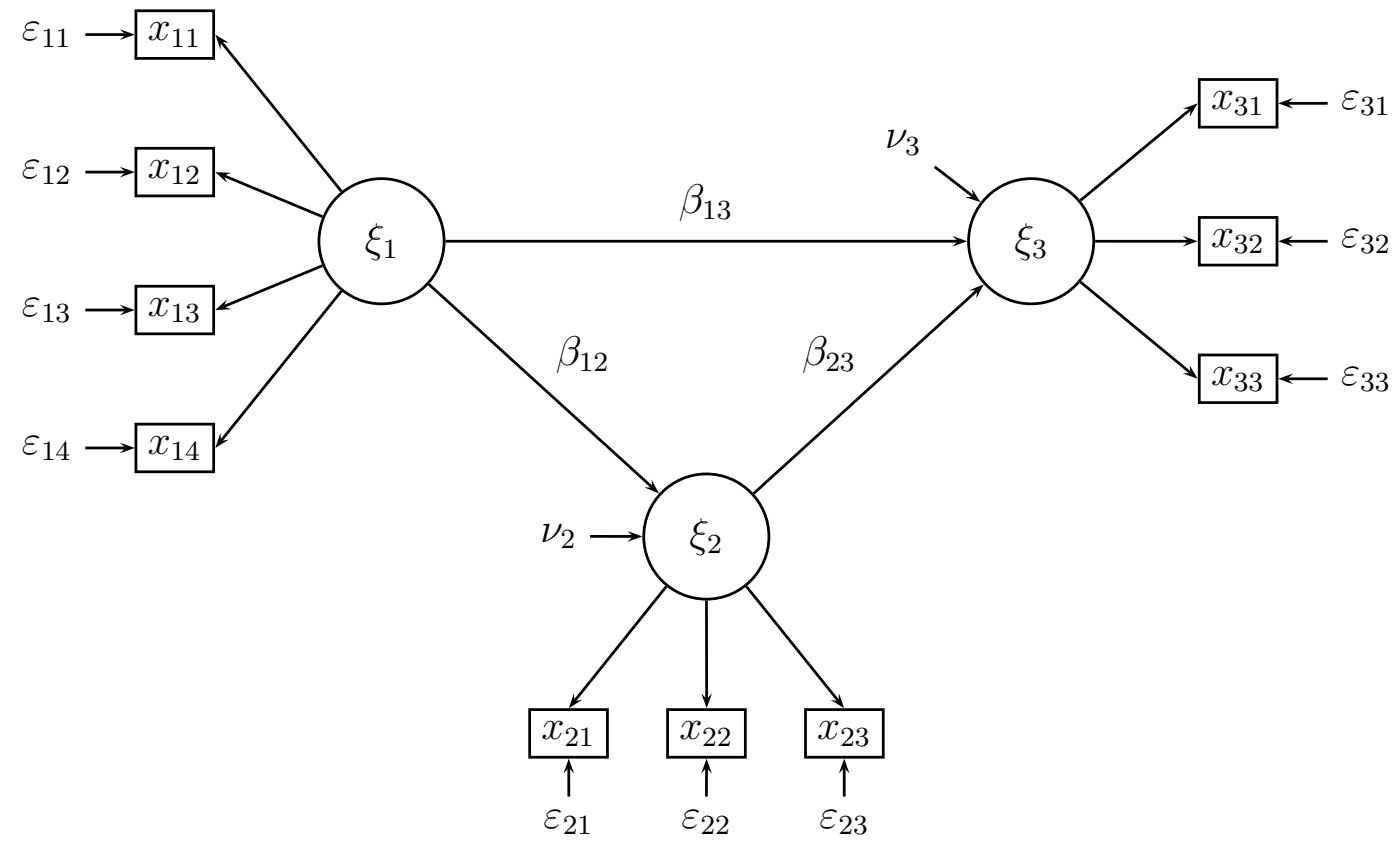

Abbildung 5.9: Beispiel für ein Modell mit drei latenten Variablen

Darstellung des Algorithmus die Annahme getroffen, dass alle manifesten Variablen standardisiert sind, d. h. einen Mittelwert von 0 und eine Standardabweichung von 1 besitzen. Eine Beschreibung des Algorithmus ohne diese Annahme findet sich z. B. bei ChATELiN ET AL. (2002, S. 8-10). Bei der Erweiterung des Algorithmus für mehr als zwei latente Variablen bedient man sich eines Tricks: Für die latenten Variablen $\xi_{j}$ wird nicht nur einer, sondern zwei (verschiedene) Schätzwerte ermittelt: ein äußerer Schätzwert $Y_{j}$ aus dem Messmodell und ein innerer Schätzwert $Z_{j}$ aus dem Strukturmodell. Abgesehen von der Initialisierung besteht der Algorithmus zur Schätzung der latenten Variablen aus zwei jeweils zweiteiligen Schritten. Iterativ wird in jedem Schritt ein Schätzwert festgehalten (abwechselnd der innere und der äußere), während (a) Gewichtungsfaktoren und (b) die jeweils anderen Schätzwerte berechnet werden. Die Schritte des Schätzalgorithmus für die latenten Variablen lauten wie folgt:

Schritt 0: Bei der Initialisierung wird ein erster äußerer Schätzwert $Y_{j}$ für jede latente Variable $\xi_{j}$ ermittelt. Im Prinzip könnten die $Y_{j}$ beliebige nichttriviale Linearkombinationen der zugehörigen manifesten Variablen sein. Aus praktischen Erwägungen schlagen Chatelin et AL. (2002, S. 9f.) als eine Möglichkeit vor, das Gewicht des ersten Indikators einer jeden latenten Variablen auf 1 und die Gewichte der übrigen 
Indikatoren auf $0 \mathrm{zu}$ setzen. $Y_{j}$ wird somit gleich der ersten manifesten Variablen gesetzt:

$$
\forall j: \quad Y_{j}:=x_{j 1}
$$

Schritt 1a: Zur Vorbereitung der inneren Schätzung der latenten Variablen dient die Schätzung der inneren Gewichte $e_{j i}$. Zur Auswahl hierfür stehen das Zentroidschema, das Faktorschema sowie das Pfadgewichtungsschema:

- Sofern das PLS-Pfadmodell Pfeile zwischen den latenten Variablen $\xi_{i}$ und $\xi_{j}$, d. h. einen Eins-Eintrag an der entsprechenden Stelle der inneren Designmatrix, aufweist, werden beim Zentroidschema die inneren Gewichte $e_{j i}$ gleich dem Vorzeichen der Korrelation zwischen $Y_{i}$ und $Y_{j}$ gesetzt, ansonsten erhalten sie den Wert 0:

$$
\forall j, i: \quad e_{j i}:= \begin{cases}\operatorname{sgn}\left(\operatorname{korr}\left(Y_{i}, Y_{j}\right)\right), & d_{i j}+d_{j i}>0 \\ 0, & \text { sonst. }\end{cases}
$$

Das Zentroidschema ist das ursprünglich von WoLD (vgl. z. B. 1982) entwickelte Schema. Es besitzt allerdings bei betragsmäßig sehr kleinen Korrelationen den Nachteil, dass sich die inneren Gewichte sprunghaft verändern können.

- Diesen Nachteil behebt das Faktorschema. Beim Faktorschema wird nicht das Vorzeichen der Korrelation als Schätzer für das innere Gewicht verwendet, sondern der Wert der Korrelation selbst:

$$
\forall j, i: \quad e_{j i}:= \begin{cases}\operatorname{korr}\left(Y_{i}, Y_{j}\right), & d_{i j}+d_{j i}>0 ; \\ 0, & \text { sonst. }\end{cases}
$$

- Eine Weiterentwicklung des Faktorschemas ist das Pfadgewichtungsschema. Für jede latente Variable $\xi_{j}$ wird unterschieden zwischen Vorgängern, d. h. latenten Variablen, von denen aus ein Pfeil auf $\xi_{j}$ zeigt, und Nachfolgern, d. h. latenten Variablen, auf die ein von $\xi_{j}$ ausgehender Pfeil zeigt. Für Nachfolger ändert sich im Vergleich zum Faktorschema nichts. Für Vorgänger $\xi_{i}$ werden die inneren Gewichte gleich den Regressionskoeffizienten $b_{i j}$ der multiplen Regression mit allen Vorgängern als unabhängigen Variablen und $\xi_{j}$ als abhängiger Variable gesetzt.

$$
\forall j, i: \quad e_{j i}:= \begin{cases}b_{i j}, & d_{i j}>0 \\ \operatorname{korr}\left(Y_{i}, Y_{j}\right), & d_{j i}>0 \\ 0, & \text { sonst. }\end{cases}
$$


Die Regressionskoeffizienten $b_{i j}$ sind zugleich die Pfadwerte des Strukturmodells, d.h. die multiple Regression, die für die Vorgänger beim Pfadgewichtungsschema benötigt wird, ist genau die gleiche wie zur Bestimmung der Strukturmodellparameter.

Schritt 1b: Bei der inneren Schätzung der latenten Variablen erhält man einen Schätzwert $Z_{j}$ für die latente Variable $\xi_{j}$ als gewichtete Summe der übrigen latenten Variablen:

$$
Z_{j}:=\varphi_{j} \cdot \sum e_{j i} Y_{i}
$$

Die Gewichte $e_{j i}$ wurden in Schritt 1a wahlweise nach dem Zentroidschema, dem Faktorschema oder dem Pfadgewichtungsschema ermittelt. Die Faktoren $\varphi_{j}$ dienen zur Standardisierung der $Z_{j}$.

Schritt 2a: Die Schätzung der äußeren Gewichte hängt maßgeblich von der Wahl des Messmodells für jede einzelne latente Variable ab. Für reflektive und formative Messmodelle hat sich jeweils eine Methode etabliert.

Bei reflektiven Messmodellen kommt gewöhnlich der sog. Modus A zum Einsatz. Beim Modus A entspricht das äußere Gewicht $\pi_{j h}$ dem Regressionskoeffizienten der einfachen Regression mit der manifesten Variablen $x_{j h}$ als abhängiger und der inneren Schätzgröße $Z_{j}$ als unabhängiger Variable:

$$
\pi_{j h}:=\operatorname{cov}\left(x_{j h}, Z_{j}\right)
$$

Da sowohl die manifesten Variablen $x_{j h}$ als auch die inneren Schätzgrößen $Z_{j}$ standardisiert sind, entspricht die Kovarianz dem besagten Regressionskoeffizienten. Auf diesem Weg erhalten diejenigen manifesten Variablen, die sich einen größeren Teil der Varianz mit der inneren Schätzgröße der latenten Variable teilen, erwartungsgemäß ein größeres Gewicht.

Bei formativen Messmodellen dominiert der sog. Modus B. Beim Modus B ergibt sich der Vektor $\pi_{j}$ der Gewichte $\pi_{j h}$ als Regressionskoeffizientenvektor aus der multiplen Regression mit der inneren Schätzgröße $Z_{j}$ als abhängiger Variable und den zur latenten Variablen $\xi_{j}$ gehörenden manifesten Variablen $x_{j h}$ als unabhängigen Variablen. Reiht man die Spaltenvektoren der manifesten Variablen $x_{j h}$ aneinander zu einer Matrix $X_{j}$, so lässt sich der Regressionskoeffizientenvektor mittels der Matrixform der multiplen Regression darstellen:

$$
\pi_{j}:=\left(X_{j}^{T} X_{j}\right)^{-1} X_{j}^{T} Z_{j}
$$


Schritt 2b: Bei der äußeren Schätzung der latenten Variablen werden alle latenten Variablen anhand der ihnen zugeordneten manifesten Variablen geschätzt. Man ermittelt äußere Schätzwerte $Y_{j}$ für die latenten Variablen $\xi_{j}$ als Linearkombinationen der manifesten Variablen:

$$
Y_{j}:=f_{j} \cdot \sum \pi_{j h} x_{j h}
$$

Die Faktoren $f_{j}$ dienen zur Standardisierung der $Y_{j}$.

Die Schritte 1a bis 2b werden so lange wiederholt, bis ein vorzugebendes Abbruchkriterium erfüllt ist. Als Abbruchkriterium dient üblicherweise die Summe der Änderung der Gewichte von einer Iteration zur nächsten. Sobald die Gewichteänderung einen bestimmten Wert (z. B. 10 ${ }^{-6}$ ) unterschreitet, bricht der Algorithmus ab. Der Algorithmus zur Schätzung der latenten Variablen endet stets mit der äußeren Schätzung der latenten Variablen. Aus den manifesten Variablen und den äußeren Gewichten kann man also die Werte der latenten Variablen auch außerhalb eines etwaigen PLS-Programms berechnen.

Die Werte für die $\xi_{j}$ stehen an dieser Stelle fest. In Abhängigkeit vom verwendeten Messmodell werden anschließend die Gewichte $\pi_{j}$ bzw. die Ladungen $\lambda_{j}$ mittels PLSRegressionen ermittelt. Während das Messmodell nun komplett bestimmt ist, fehlt noch die Schätzung des Strukturmodells. Dies geschieht mit Hilfe multipler Regressionen: Für jede endogene latente Variable $\xi_{j}$ werden die Pfadwerte $\beta_{i j}$ als Regressionskoeffizienten aus der Regression mit $\xi_{j}$ als abhängiger Variable und all ihren Vorgängern $\xi_{i}$ als unabhängigen Variablen berechnet.

\subsubsection{Schätzung von Modellen mit Konstrukten zweiter Ordnung}

Laut Wold (1982, S. 40f.) lassen sich Modelle, die latente Variablen mit hierarchischer Struktur - darunter fallen insbesondere Konstrukte zweiter Ordnung - enthalten, ebenfalls mit dem PLS-Algorithmus schätzen, ohne dass Modifikationen am Algorithmus vorzunehmen wären.

Um ein Modell mit Konstrukten zweiter Ordnung zu schätzen, werden dem Konstrukt der zweiten Ebene erneut alle die Indikatoren zugewiesen, die mit den zugehörigen Konstrukten der ersten Ebene verbunden sind. Das Beispiel für ein Messmodell eines Konstrukts zweiter Ordnung des Typs II in Abb. 5.10 verdeutlicht dieses Vorgehen.

Während sich bei der Kovarianzstrukturanalyse Konstrukte zweiter Ordnung durch sog. „Phantomvariablen“ ohne zugehörige Indikatoren modellieren lassen, können bei der PLS-Pfadmodellierung latente Variablen ohne eigene Indikatoren nicht geschätzt werden. 


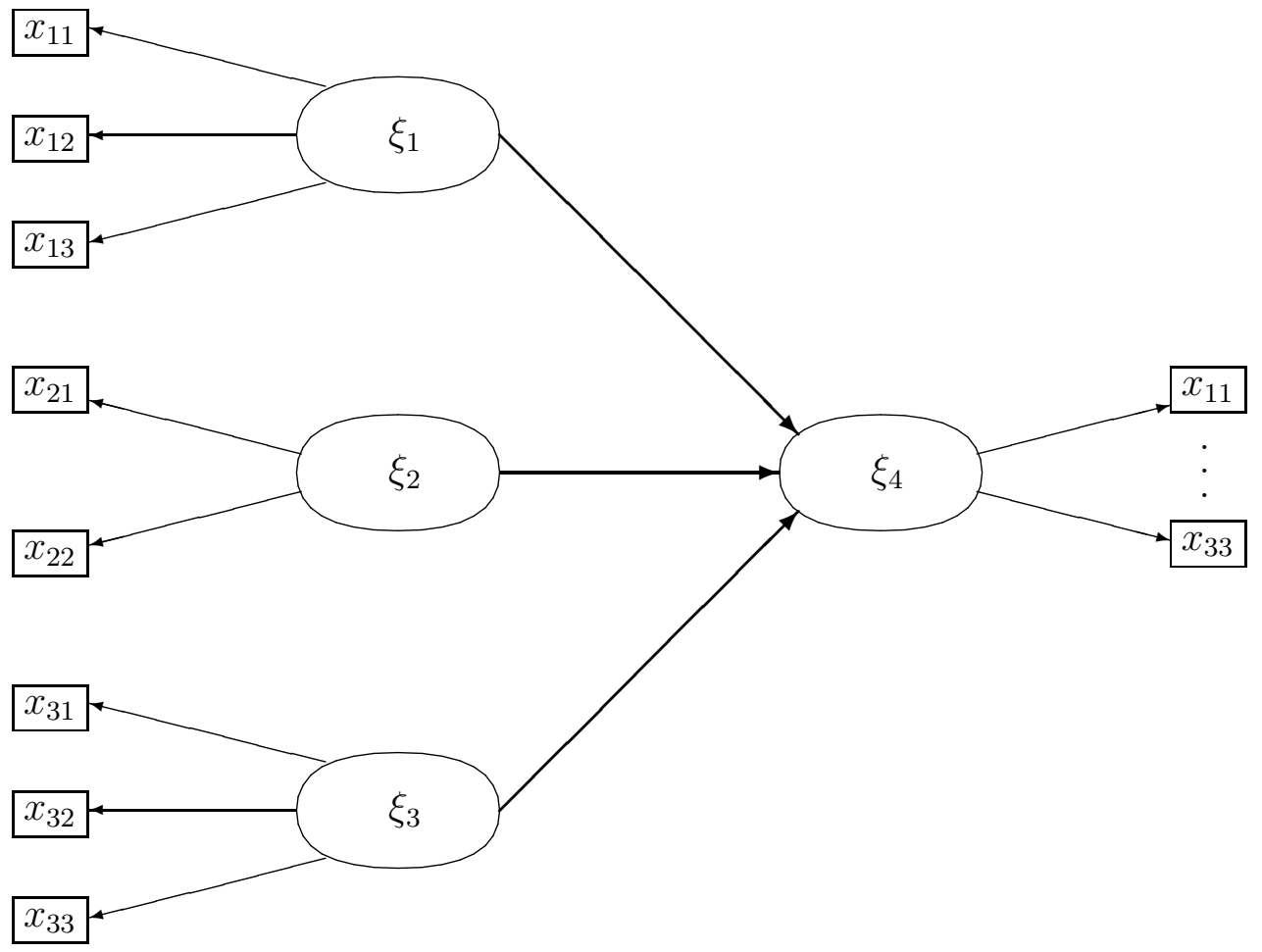

Abbildung 5.10: Beispiel für die Modellierung von Konstrukten zweiter Ordnung mittels PLS-Pfadmodellierung (angelehnt an WoLD, 1982, S. 41)

Schließlich werden bei der PLS-Pfadmodellierung die latenten Variablen explizit als Linearkombination ihrer Indikatoren berechnet. Die Lösung, die Indikatoren der Konstrukte erster Ordnung wiederum für die Konstrukte zweiter Ordnung zu verwenden, ist nicht nur aus mathematischer, sondern auch aus inhaltlicher Perspektive sinnvoll: Die Nähe des Konstrukts zweiter Ordnung zum eigentlichen Messinstrument, den Indikatoren, erleichtert die inhaltliche Interpretation.

\subsubsection{Schätzung von Modellen mit moderierenden Effekten}

Generell erweisen sich kovarianzstrukturbasierte Verfahren wie z. B. LISREL oder AMOS als wenig geeignet, moderierende Effekte nachzuweisen, da sie erhebliche Anforderungen an das Datenmaterial, die Rechnerkapazität und die Samplegröße stellen (CHIN ET AL., 2003, S. 194ff.). Für die Schätzung von moderierenden Effekten mittels PLSPfadmodellierung existieren in Abhängigkeit von den verwendeten Messmodellen unterschiedliche Anwendungsempfehlungen: 
Bei reflektiven Indikatoren bzw. Konstrukten werden zur Untersuchung des moderierenden Zusammenhangs die einzelnen Indikatoren der Konstrukte (Prädiktor und Moderator) multipliziert und zu einem neuen Konstrukt, der Interaktionsvariable, zusammengefasst. Eine Anwendung hierfür demonstrieren z. B. ChIn ET AL. (2003, S. 199ff.).

Anders ist bei formativen Konstrukten zu verfahren: „Since formative indicators are not assumed to reflect the same underlying construct (i. e., can be independent of one another and measuring different factors), the product indicators between two sets of formative indicators will not necessarily tap into the same underlying interaction effect" (CHIN ET AL., 2003, S. 11 (Supplement)). Helm ET AL. (2005, im Druck) empfehlen daher ein Vorgehen in drei Schritten:

1. Im ersten Schritt werden mittels PLS aus den Indikatoren des Prädiktors und des Moderators die jeweiligen Konstruktwerte berechnet.

2. Im zweiten Schritt werden diese Konstruktwerte elementweise miteinander multipliziert. Das Ergebnis bezeichnen HELM ET AL. (2005) als Indikatorvariable.

3. Im dritten Schritt geht die Indikatorvariable als Single-Item-Variable gemeinsam mit dem Prädiktor und dem Moderator in das PLS-Modell ein, das nun geschätzt werden kann.

Auf diese Weise lässt sich die Indikatorvariable neben den beiden Originalkonstrukten in ihrem Einfluss auf die abhängige Variable prüfen.

Bei den hier vorgestellten Handlungsempfehlungen zur Handhabung von moderierenden Variablen in PLS-Pfadmodellen handelt es sich um den State-of-the-Art. Jedoch weisen sie einige Probleme auf, als da sind:

1. die Diskrepanz zwischen den Werten der Interaktionsvariablen und dem elementweisen Produkt aus Prädiktor und Moderator,

2. die Diskrepanz zwischen den Werten der Indikatorvariablen und dem elementweisen Produkt aus Prädiktor und Moderator,

3. die unklare Handhabung von Konstrukten zweiter Ordnung sowie

4. die fehlende Empfehlung für den Fall, dass der Prädiktor und der Moderator unterschiedlich konzeptualisiert sind.

Diese Probleme werden im Folgenden kurz erläutert. 
Das erste Problem bezieht sich auf den Fall, wenn sowohl der Prädiktor als auch der Moderator reflektiv konzeptualisert sind. In diesem Fall stimmen bei der Verwendung der Methode von ChIn ET AL. (2003) die Werte der Interaktionsvariablen gewöhnlich nicht mit dem elementweisen Produkt der Konstruktwerte von Prädiktor und Moderator überein. Bei der abschließenden Regression innerhalb der Modellschätzung zur Ermittlung der Pfadkoeffizienten handelt es sich bei der Interaktionsvariablen also nicht um einen echten Produktterm, wie er gewöhnlich in Regressionsanalysen zur Überprüfung von Interaktionseffekten eingesetzt wird, sondern allenfalls um einen Pseudoproduktterm.

Das zweite Problem tritt auf, wenn es sich sowohl beim Prädiktor als auch beim Moderator um ein formatives Konstrukt handelt. Auch in diesem Fall kann zwischen den Werten der Indikatorvariable und dem elementweisen Produkt der Faktorwerte von Prädiktor und Moderator eine Diskrepanz entstehen, und zwar dann, wenn im oben beschriebenen dritten Schritt die Gewichte der formativen Konstrukte neu ermittelt werden. Hierdurch ändern sich die Werte der formativen Konstrukte, sodass ihr elementweises Produkt nicht mehr der Indikatorvariablen entspricht. Diese Diskrepanz dürfte im Durchschnitt der Fälle größer sein als die Diskrepanz zwischen den Werten der Interaktionsvariablen und dem elementweisen Produkt aus Prädiktor und Moderator, da formative Indikatoren nicht miteinander korrelieren müssen und aus diesem Grunde ein formatives Konstrukt in einem modifizierten Modell stark veränderte Konstruktwerte aufweisen kann. Man kann zwar die Diskrepanz vermeiden, indem man im dritten Schritt an Stelle eines PLS-Pfadmodells eine gewöhnliche multiple Regression mit den Konstruktwerten rechnet. In diesem Fall wird jedoch die zur Verfügung stehende Information nicht in vollem Maße genutzt, was zu verzerrten Schätzern für die Pfadkoeffizienten führen kann.

Ein drittes Problem tritt auf, will man Konstrukte zweiter Ordnung als Prädiktoren oder Moderatoren in ein Modell integrieren. Hierfür gibt es in der Literatur keine Handlungsempfehlungen. Die Frage nach der Behandlung von Konstrukten zweiter Ordnung gewinnt an Brisanz durch die verschiedenen Möglichkeiten der Konzeptualisierung, vgl. Unterabschnitt 5.1.3 und insbesondere Abb. 5.5.

Ein viertes Problem entsteht, wenn der Prädiktor formativ und der Moderator reflektiv konzeptualisiert ist oder umgekehrt. Auch für diesen Fall gibt die Literatur keine Empfehlung.

Die genannten Probleme könnten dazu führen, dass für die Höhe der Interak-tionseffekte entweder fehlerhafte Schätzwerte ermittelt werden oder die simultane Überprüfung aller Hypothesen gänzlich unmöglich wird. Als Reaktion auf diese Probleme wurde im Rahmen dieser Arbeit eine neue Form der Integration moderierender Effekte in PLSPfadmodelle entwickelt. Den Ausgangspunkt bildet ein Vorteil der Partial-Least-Squares- 
Pfadmodellierung gegenüber kovarianzbasierten Verfahren: die explizite Berechnung der Werte der latenten Variablen - sowohl am Ende des Schätzalgorithmus als auch in seinem Verlauf.

Hierzu wird im Schätzalgorithmus ein Schritt 2c zur Schätzung der Werte der Interaktionsvariablen hinzugefügt. Sobald alle latenten Variablen eine äußere Schätzung erfahren haben, werden die Werte der Interaktionsvariablen als elementweises Produkt (Operator: *) der Werte des jeweils zugehörigen Prädiktors und Moderators berechnet:

$$
Y_{\text {Interaktion }}:=f \cdot Y_{\text {Prädiktor }} * Y_{\text {Moderator }}
$$

Der Faktor $f$ dient dazu, die Varianz der Interaktionsvariable $Y_{\text {Interaktion }}$ auf den Wert 1 zu standardisieren. Der Algorithmus wurde in der Programmiersprache $R$ implementiert.

Zwar löst die neu vorgestellte Methode die oben thematisierten Probleme, sie hat allerdings auch eine Einschränkung: Zur Modellschätzung ist es zwingend erforderlich, dass im Modell sowohl der Prädiktor als auch der Moderator eine direkte Beziehung zur gemeinsamen zu erklärenden Variable aufweisen. Andernfalls lässt sich das Modell nicht schätzen.

\subsection{Beurteilung und Validierung von PLS-Pfadmodellen}

Im Rahmen der Schätzung von Kausalmodellen kommt der Modellbeurteilung und validierung eine zentrale Bedeutung zu. Im Vergleich zur Kovarianzstrukturanalyse steht bei der PLS-Pfadanalyse jedoch eine wesentlich geringere Anzahl von Gütemaßen zur Verfügung. Ursache hierfür ist der Verzicht auf Verteilungsannahmen bei PLSPfadmodellen, weshalb sich parametrisch ausgerichtete Techniken für Signifikanztests zur Modellbeurteilung nicht eignen. Wold (1980, S. 232) empfiehlt den Einsatz von Tests, die berücksichtigen, dass die PLS-Pfadmodellierung ohne Verteilungsannahmen arbeitet. Geeignet sind schätzungsorientierte, nicht-parametrische Gütemaße.

\subsubsection{Bestimmtheitsmaß und Effektgröße}

Ausgangspunkt für die Evaluierung von PLS-Pfadmodellen bilden die Bestimmtheitsmaße $R^{2}$ der einzelnen endogenen latenten Variablen aus den Regressionen des Strukturmodells. Sie lassen sich genauso interpretieren wie bei gewöhnlichen statistischen Regressionsanalysen. Das Bestimmtheitsmaß $R^{2}$ besagt, welchen Anteil die durch die (ggf. multiple) Regression des Pfadmodells erklärte Abweichungsquadratsumme an der zu erklärenden 
Abweichungsquadratsumme besitzt. Das Bestimmtheitsmaß ist das zentrale Gütekriterium der modellinternen Regressionsfunktionen an die empirischen Daten.

Des Weiteren lässt sich überprüfen, ob eine unabhängige latente Variable einen substanziellen Einfluss auf eine abhängige latente Variable ausübt. CHIN (1998b, S. 316) betrachtet hierzu die Effektgröße $f^{2}$, die sich aus den unterschiedlichen $R^{2}$ berechnet, wenn die betreffende unabhängige latente Variable in die abhängige latente Variable einfließt $\left(R_{i n k l}^{2}\right)$ oder nicht $\left(R_{e x k l}^{2}\right)$ :

$$
f^{2}:=\frac{R_{i n k l}^{2}-R_{e x k l}^{2}}{1-R_{i n k l}^{2}}
$$

In Anlehnung an die Einteilung von Cohen (1988, S. 412ff.) besagen Werte für $f^{2}$ von über $0,02 / 0,15 / 0,35$, dass die betreffende unabhängige latente Variable einen kleinen/ mittleren/großen Einfluss auf die abhängige latente Variable hat.

Vergleichsweise kleine Effektgrößen sind eher die Regel als die Ausnahme in vielen Gebieten verhaltenswissenschaftlicher Forschung: „Small effect sizes are not unique to consumer behavior research; they are endemic to behavioral research in general" (PETERSON ET AL., 1985, S. 101).

\subsubsection{Pfadkoeffizienten}

Die Pfadkoeffizienten können als $\beta$-Schätzer einer gewöhnlichen multiplen Regression der Form $y=X \cdot \beta+\varepsilon$ angesehen werden. Dies überrascht nicht, da sie im letzten Schritt der Partial-Least-Squares-Schätzung mittels einer multiplen Regression berechnet wurden.

Sowohl das Vorzeichen als auch der Betrag lassen sich sinnvoll wie zu erwarten interpretieren:

- Das Vorzeichen der Pfadkoeffizienten gibt an, ob eine Erhöhung der exogenen Variable mit einer Erhöhung (positives Vorzeichen) oder Senkung (negatives Vorzeichen) der endogenen Variablen einhergeht. Es lässt sich sehr schnell überprüfen, ob das Vorzeichen in Einklang mit der hypothetischen Wirkweise steht.

- Der Betrag der Pfadkoeffizienten steht für die Stärke der Wirkbeziehung. Erhöht man die betrachtete exogene Variable um eine Einheit (während etwaige andere exogene Variablen konstant bleiben), so ist zu erwarten, dass sich die endogene Variable um schätzungsweise den Pfadkoeffizienten mal die Einheit verändert.

Die Analyse der Pfadkoeffizienten allein genügt noch nicht zum Bestätigen oder Verwerfen der zu testenden Hypothesen. Mit der hierzu benötigten Untersuchung der Irrtumswahrscheinlichkeiten beschäftigt sich der folgende Unterabschnitt. 


\subsubsection{Bootstrapping}

Im Rahmen der Analyse stellt sich die Frage, ob die ermittelten Parameter für die gerichteten Beziehungen zwischen den latenten Variablen statistisch signifikant sind. Eine elegante Methode hierfür ist das sog. Bootstrapping. Es handelt sich hierbei um ein nichtparametrisches Verfahren, d.h. die Beurteilung der Pfadkoeffizienten hinsichtlich ihrer Signifikanz benötigt auf diese Weise keine Verteilungsannahmen.

Das Funktionsprinzip des Bootstrapping ist einfach: Aus den vorhandenen $n$ Beobachtungen werden $k$ Subsamples kreiert, indem für jedes Subsample zufällig mit Zurücklegen $n$ (auch andere Zahlen sind möglich) Beobachtungen aus dem Originalsample gezogen werden. Typische Größen für $k$ sind hierbei 100 oder 200. Für jedes Subsample wird eine Modellschätzung vorgenommen. Über alle Subsamples lassen sich nun die Erwartungswerte und Standardabweichungen sämtlicher Modellparameter bilden. Eine ausführlichere Erläuterung des Bootstrapping liefern EFron und TiBshirani (1993).

Die Quotienten aus den Pfadkoeffizienten des Originalmodells und den mittels Bootstrapping ermittelten Standardabweichungen stellen Student-t-Quantile dar. Mit ihrer Hilfe lassen sich die Konfidenzintervalle und die Signifikanzen bestimmen: Das Konfidenzintervall sagt aus, in welchem Bereich sich der wahre Parameterwert mit definierter (üblicherweise 95-prozentiger) Wahrscheinlichkeit befindet. Die Signifikanz gibt an, wie groß die Wahrscheinlichkeit ist, dass die Gegenhypothese fälschlicherweise verworfen wurde.

Wenn die Signifikanz unter einem bestimmten Wert liegt, ist die Gegenhypothese zu verwerfen, d. h. die eigentlich interessierende Hypothese wird angenommen. Anderenfalls geht man weiterhin davon aus, dass die Gegenhypothese wahr ist.

\subsection{Evaluierung und Validierung von Messmodellen}

Die Qualität der Erfassung eines Konstrukts über beobachtbare Variablen hängt im wesentlichen von der Reliabilität und der Validität der vorangegangenen Messung ab. Die Bedeutung von validen Messungen hebt PETER (1979, S. 6) hervor: „Valid measurement is the sine qua non of science. [...] If the measures used in a discipline have not been demonstrated to have a high degree of validity, that discipline is not a science."

Zunächst werden verschiedene Verfahren zur Überprüfung der Reliabilität vorgestellt (Unterabschnitt 5.4.1), bevor dann auf Methoden zur Analyse der Validität eingegangen wird (Unterabschnitt 5.4.2). 


\subsection{1 Überprüfung der Reliabilität von Messmodellen}

Eine zentrale Größe zur Beurteilung des Messmodells ist die Reliabilität. Reliabilität ist eine notwendige, allerdings nicht hinreichende Bedingung für die Validität eines Messmodells (PETER, 1979, S. 6). Ein Messmodell gilt als reliabel, wenn sich bei mehrfacher Messung derselben Merkmale bei denselben Merkmalsträgern dieselben Messwerte reproduzieren lassen.

Reliabilität, d.h. Zuverlässigkeit, ist definiert als der Grad, zu dem Messungen frei von Zufallsfehlern sind; daher schätzen eigens hierfür entwickelte Reliabilitätskoeffizienten den Betrag der systematischen Varianz einer Messung (Peter und Churchill, 1986, S. 4). Eine Messung für ein Objekt oder eine Person lässt sich als Funktion von drei Komponenten auffassen:

$$
x_{\text {beobachtet }}=x_{\text {wahr }}+\varepsilon_{\text {systematisch }}+\varepsilon_{\text {zufällig }}
$$

Die Formel besagt, dass ein beobachteter Wert eine Überlagerung von drei einzelnen Werten darstellt: erstens dem wahren Wert, zweitens einem systematischen Fehler und drittens einem Zufallsfehler. Einzelne Indikatoren führen dann zu reliablen Messungen des zugehörigen Konstrukts, wenn ein wesentlicher Anteil ihrer Varianz durch die Assoziationen mit dem Konstrukt erklärt wird bzw. wenn die Messfehlervariablen $\varepsilon_{\text {systematisch }}$

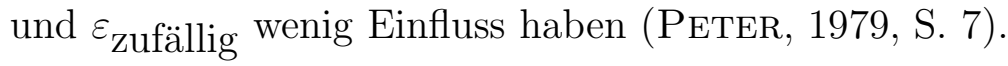

Zur Beurteilung reflektiver Messmodelle eignen sich die vom statistischen Verfahren der Faktorenanalyse her bekannten Maße zur Beurteilung der Indikator-, der internen Konsistenz- und der Faktorreliabilität (Homburg und Baumgartner, 1998, S. 360ff.).

\section{Indikatorreliabilität}

Für jeden Indikator lässt sich die Indikatorreliabilität ermitteln. Sie entspricht dem Bestimmtheitsmaß $R^{2}$ aus der Regression mit dem Indikator als abhängiger und der zugehörigen latenten Variable als unabhängiger Größe. Die Indikatorreliabilität gibt an, welchen Anteil der Indikatorvarianz der Indikator mit der zugehörigen latenten Variable gemeinsam hat. Sind sowohl die latente Variable als auch ihre Indikatoren standardisiert, entspricht die Indikatorreliabilität dem Quadrat der Indikatorladung, $\lambda^{2}$.

Der kritische Wert der Indikatorreliabilität variiert mit der Stichprobengröße. Während bei einer Stichprobe mit einem Umfang zwischen 100 und 400 Versuchspersonen eine Indikatorreliabilität zwischen 0,4 und 0,6 ausreicht, sind bei Stichprobenumfängen zwischen 400 und 1000 bereits Werte zwischen 0,2 und 0,4 akzeptabel (BALDERJAHN, 1986, S. 117). 


\section{Interne Konsistenzreliabilität}

Ein Maß für die interne Konsistenz der Indikatoren eines Faktors ist das sog. Cronbach's Alpha. Es dient zur Messung der Reliabilität einer Gruppe von Indikatoren, die zusammen ein Konstrukt messen sollen. Es ist der wohl am häufigsten angewandte Reliabilitätskoeffizient. Cronbach's Alpha wird nach der folgenden Formel bestimmt (CRONBACH, 1951, S. 305):

$$
\alpha=\frac{n}{n-1} \cdot \frac{\sum_{g} \sum_{h} \operatorname{cov}\left(x_{g}, x_{h}\right)}{\operatorname{var}\left(\sum_{h} x_{h}\right)} ; \quad(g, h=1,2, \ldots, H ; g \neq h) .
$$

Cronbach's Alpha nimmt Werte kleiner gleich Eins an; es sind auch negative Werte möglich. Ein Wert nahe Eins repräsentiert hohe Reliabilität. NunNALLY (1978, S. 245) empfiehlt, insbesondere in frühen Forschungsphasen mit einem Alphawert ab 0,7 zu arbeiten.

Einerseits erfreut sich unter Forschern die Beurteilung der Reliabilität anhand des Cronbach's Alphas einer hohen Beliebtheit. Andererseits ist Cronbach's Alpha mit einigen Einschränkungen behaftet, die insbesondere durch die sehr restriktiven Annahmen begründet sind. Das Cronbach's Alpha geht von zwei Prämissen aus (Gerbing und Anderson, 1988, S. 190):

- Die Indikatoren stellen einen eindimensionalen Satz dar.

- Alle Indikatoren eines Faktors weisen die gleiche Reliabilität auf.

Noch zwei weitere Nachteile beeinträchtigen die Aussagekraft des Cronbach's Alphas. Zum einen ist die Höhe des Wertes positiv abhängig von der Anzahl an Indikatoren (CoRTINA, 1993, S. 101), und zum anderen wird auf eine inferenzstatistische Beurteilung meistens verzichtet, obwohl entsprechende Test entgegen vielbeachteter anderslautender Meinungen (Homburg und Giering, 1996, z. B.) existieren.

\section{Faktorreliabilität}

Mittels der Faktorreliabilität lässt sich beurteilen, inwieweit ein Faktor den ihm zugeordneten Block reflektiver Indikatoren zu erklären vermag. Sofern man in einem PLSPfadmodell ausschließlich mit standardisierten Indikatorvariablen arbeitet, lässt sich die Faktorreliabilität $\rho_{c}$ über die folgende Gleichung berechnen:

$$
\rho_{c}=\frac{\left(\sum_{i} \lambda_{i}\right)^{2}}{\left(\sum_{i} \lambda_{i}\right)^{2}+\sum_{i} \operatorname{var}\left(\varepsilon_{i}\right)} \quad \text { mit } \quad \operatorname{var}\left(\varepsilon_{i}\right)=1-\lambda_{i}^{2}
$$


Hierbei ist $\lambda_{i}$ die Indikatorladung und $\varepsilon_{i}$ der Residuenvektor des $i$-ten Indikators des betrachteten Faktors. CHIn (1998a, S. 320) vergleicht Cronbach's Alpha mit der Faktorreliabilität $\rho_{c}$ und kommt dabei zu dem Schluss, dass die Faktorreliabilität „, [.. ] does not assume tau equivalency among the measures with its assumption that all indicators are equally weighted. Therefore, alpha tends to be a lower bound estimate of reliability, whereas $\rho_{c}$ is a closer approximation under the assumption that the parameter estimates are accurate." Die Werte für $\rho_{c}$ können zwischen null und eins liegen. In der Literatur werden Werte von unter 0,6 als Zeichen für mangelnde Reliabilität interpretiert (HomBURG und BAUMGarTner, 1998, S. 361).

\subsection{2 Überprüfung der Validität von Messmodellen}

Die Validität charakterisiert die konzeptionelle Richtigkeit eines Messmodells, d.h. ein Messmodell ist genau dann valide, wenn es wirklich das misst, was es messen soll (HEELER und RAY, 1972, S. 361). Validität liegt vor, wenn die Unterschiede in den beobachteten Werten auch wirkliche Unterschiede des Phänomens, welches gemessen werden soll, repräsentieren (CHuRCHILl, 1979, S. 65). Vier Formen von Validität werden im Folgenden vorgestellt: die Konstruktvalidität, die Konvergenzvalidität, die Diskriminanzvalidität sowie die externe Validität.

\section{Konstruktvalidität}

Konstruktvalidität bezieht sich auf das Ausmaß, in dem die zu betrachtenden hypothetischen, unbeobachtbaren Konstrukte mit ihren jeweiligen Messungen korrespondieren. PETER (1981, S. 133) merkt an, dass ,[a] basic goal of social science is to provide theoretical explanations of behavior. In marketing, this goal includes attempts to explain the behavior of consumers, salespersons, and others involved in discipline-related activities. [...] Because construct validity pertains to the degree of correspondence between constructs and their measures, construct validity is a necessary condition for theory development and testing." Auf gleiche Weise argumentieren Anderson und Gerbing (1982, S. 453) mit ihrer Aussage, dass "the reason for drawing a distinction between the measurement model and the structural model is that proper specification of the measurement model is necessary before meaning can be assigned to the analysis of the structural model" sowie BAGOzZI (1981, S. 376), indem er fordert, dass „convergence in measurement should be considered a criterion to apply before performing the causal analysis because it represents a condition that must be satisfied as a matter of logical necessity." 
Damit ein Messmodell konstruktvalide ist, muss sich jeder der Indikatoren zum einen auf die Charakteristika des jeweils zugeordneten Konstrukts beziehen und zum anderen frei von Kontamination durch Elemente anderer Konstrukte sein (PETER, 1981, S. 134). Diese beiden Anforderungen lassen sich durch zwei Validitätstests operationalisieren:

1. Die Inhaltsvalidität ist der Grad, zu dem ein Messmodell das Konstrukt wie definiert widerspiegelt. Die Inhaltsvalidität lässt sich vor der Messung beurteilen; sie basiert auf dem intuitiven Urteil des Forschers oder von Experten (MitTaL, 1995, S. 665). Eine profunde Kenntnis des Konstruktinhalts ist hierbei unabdingbar.

2. Unidimensionalität bedeutet, dass das Messinstrument weitgehend frei von Kontamination durch Elemente anderer Konstrukte ist. Unidimensionalität lässt sich mittels einer Faktorenanalyse überprüfen: Sie liegt dann vor, wenn die Hauptkomponente der Indikatoren eines reflektiv operationalisierten Konstrukts mehr als die Hälfte der Varianz dieser Indikatoren erklärt.

\section{Konvergenzvalidität}

Die Konvergenzvalidität besagt, inwieweit unterschiedliche Messungen eines Konstrukts zum gleichen Ergebnis gelangen. Sie ist vom Wesen her identisch mit der Faktorreliabilität (ForNell und LARCKER, 1981, S. 45), und kann daher auch anhand von $\rho_{c}$ ausgedrückt werden. Ein weiteres Maß zur Beurteilung der Konvergenzvalidität ist die durchschnittlich erfasste Varianz (engl.: average variance extracted, Abk.: AVE). Sie bestimmt sich nach der folgenden Formel:

$$
\mathrm{AVE}=\frac{\sum_{i} \lambda_{i}^{2}}{\sum_{i} \lambda_{i}^{2}+\sum_{i} \operatorname{var}\left(\varepsilon_{i}\right)}
$$

Auch hier ist $\lambda_{i}$ die Indikatorladung und $\varepsilon_{i}$ der Residuenvektor des $i$-ten Indikators des betrachteten Faktors. CHIN (1998a, S. 321) merkt an, dass „[w]hen all indicators are standardized, this measure would be the same as the average of the communalities in the block." 'In diesem Fall lässt sich die Formel 5.17 unter Zuhilfenahme der Eigenschaft $\operatorname{var}\left(\varepsilon_{i}\right)=1-\lambda^{2}$ vereinfachen $\mathrm{zu}$ :

$$
\mathrm{AVE}=\frac{1}{n} \sum_{i} \lambda_{i}^{2}
$$

$n$ ist hierbei die Anzahl der Indikatoren des betrachteten Faktors. Die Herkunft des Namens wird aus der Formel 5.18 unmittelbar ersichtlich. Die AVE kann Werte zwischen 
null und eins annehmen. Fornell und LARCKER (1981, S. 46) fordern für die AVE einen Schwellenwert von 0,5, ab dem von einem akzeptablen Messmodell gesprochen werden kann. Anderenfalls wäre die auf Messfehlern beruhende Varianz größer als die Varianz, die durch das Konstrukt hervorgerufen wird. Die AVE stellt zugleich ein konservativeres Maß für die Faktorreliabilität dar als $\rho_{c}$ (ForNELL und LARCKER, 1981, S. 46).

\section{Diskriminanzvalidität}

Ein weiterer Schritt zur Validierung des Messmodells ist die Überprüfung der Diskriminanzvalidität. Diskriminanzvalidität liegt dann vor, wenn die Indikatoren eines reflektiven Konstrukts deutlich stärker auf das zugehörige Konstrukt als auf die übrigen Konstrukte laden. Insbesondere zwei Methoden zur Überprüfung der Diskriminanzvalidität haben sich etabliert: Die Betrachtung der durchschnittlich erfassten Varianz sowie die exploratorische Faktorenanalyse.

Ist die durchschnittlich erfasste Varianz größer als die quadrierten Korrelationen eines Konstrukts mit allen anderen Konstrukten, so gilt dies als ein Zeichen für die diskriminanzvalide Messung eines Konstrukts (ForNell und LARCKER, 1981, S. 46).

Aufgabe der exploratorischen Faktorenanalyse ist es, die vorliegenden Indikatoren bezüglich der ihnen zugrunde liegenden Faktorenstruktur zu untersuchen, wobei hierbei noch keine Hypothesen über die Zuordnung der Faktoren untereinander vorliegen (Backhaus et AL., 2003, S. 330). Nach der mittels dieses Verfahrens erreichten Verdichtung der Indikatoren zu Faktoren kann von einem sinnvollen Ausmaß an Konvergenz- und Diskriminanzvalidität gesprochen werden, wenn sich alle Indikatoren eines Konstrukts genau einem Faktor zuordnen lassen. Möglich ist dies dann, wenn die Indikatoren eines Faktors ausreichende Faktorladungen aufweisen (bspw. >0,5) und auf die anderen Faktoren deutlich niedriger laden.

\section{Externe Validität}

Auf formative Messmodelle lassen sich die vorgenannten Werkzeuge zur Validierung von Messmodellen nicht anwenden. REINARTz ET AL. (2004) schlagen daher vor, ersatzweise die externe Validität zur Gütebeurteilung zu verwenden.

Entgegen der Annahme der PLS-Pfadmodellierung, dass formative Messmodelle messfehlerfrei sind, ist eine fehlerfreie und vollständige Erfassung in der Realität nur selten möglich. Wie sich am Beispiel des Konstrukts Trunkenheit aufzeigen lässt (s. Abb. 5.4 auf S. 97), kann es zu Differenzen zwischen einer formativen und einer reflektiven Messung 
kommen, wenn potenzielle Ursachen des formativ gemessenen Konstrukts, wie im konkreten Fall der Trunkenheit bspw. alkoholhaltige Pralinen, außer Acht gelassen werden. Die Größe dieses Unterschieds zwischen formativen und reflektiven Messungen lässt sich als Validitätskriterium ansehen.

Diamantopoulos und Winklhofer (2001, S. 272-274) empfehlen, ein Zwei-KonstruktModell (Pseudo-MIMIC-Modell) zu bilden, dessen erstes Konstrukt formativ und dessen zweites Konstrukt reflektiv gemessen wird. Zeigt sich zwischen den beiden Konstrukten ein starker und signifikanter Zusammenhang, liegt externe Validität vor (RINDskopf, 1984). 


\section{Empirische Untersuchung des Wechselverhaltens von Konsumenten}

Um das in Kapitel 4 entwickelte Hypothesensystem zum Wechselverhalten von Konsumenten im Strommarkt einem Falsifikationsversuch zu unterziehen, wurde eine empirische Untersuchung konzipiert und im Strommarkt für Haushaltskunden durchgeführt.

Nach einer kurzen Darstellung des Untersuchungsziels in Abschnitt 6.1 beschreibt Abschnitt 6.2 das Erhebungsdesign. Hieran schließt sich Abschnitt 6.3 mit der Operationalisierung der in Kapitel 4 konzeptualisierten Konstrukte an. Den Inhalt von Abschnitt 6.4 bildet die Analyse des Messmodells. Sodann widmet sich Abschnitt 6.5 der Auswertung des Strukturmodells und damit dem eigentlichen Test des Hypothesensystems. Abschnitt 6.6 geht darauf ein, inwieweit sich der Einfluss der Antezedenzvariablen von Wechselabsicht und Wechselvollzug unterscheidet.

\subsection{Ziel der Untersuchung und Abgrenzung des Untersuchungsobjekts}

Allgemein soll die empirische Untersuchung offenbaren, ob das in Kapitel 4 entwickelte Modell zum Wechselverhalten von Konsumenten im Strommarkt die Realität in adäquatem Maße abbildet. Die Untersuchung verfolgt dabei sowohl einen konfirmatorischen als auch einen explorativen Ansatz:

Einerseits sollen die Hypothesen, die in Kapitel 4 ausgehend von Theorien oder Analogien aus anderen Bereichen hergeleitet wurden, überprüft werden. Dieses strukturprüfende Vorgehen schließt auch die Untersuchung der konkurrierenden Hypothesen zum direkten Einfluss des Involvements mit ein.

Andererseits geht es auch darum, Strukturen zu entdecken: Eine mögliche Diskrepanz zwischen den relevanten Einflussgrößen von Wechselabsicht und Wechselvollzug soll Aufschluss darüber geben, warum Konsumenten ihren Anbieter nicht wechseln, selbst wenn sie die Absicht dazu haben. 


\subsection{Erhebungsdesign}

Ein Hypothesensystem wird getestet, indem man es mit der Realität konfrontiert. Damit dieser Test für Dritte nachvollziehbar ist, sollte der Aufbau der empirischen Untersuchung offengelegt werden.

In den Zeiträumen 6. bis 22. Januar 2004 und 3. bis 23. April 2004 wurden Telefoninterviews mit Haushaltsstromkunden geführt. Die Wahl der Erhebungsform fiel aus mehreren Gründen auf eine Telefonbefragung: Eine persönliche Befragung wäre aufgrund des dafür notwendigen Aufwands und der möglichen Durchführungsprobleme eher ungeeignet gewesen. Eine schriftliche Befragung zum Wechselverhalten im Strommarkt ist zwar wirtschaftlicher in der Durchführung, wäre aber wahrscheinlich dem self-selection-effect ausgesetzt gewesen: Es kann nicht ausgeschlossen werden, dass hauptsächlich Personen einen Fragebogen ausfüllen und zurückschicken würden, die sich für das Thema Strom interessieren, d.h. ein überdurchschnittliches Involvement besitzen. Mit der Telefonbefragung wurde eine Erhebungsmethode gewählt, die durch eine hohe externe Validität, recht hohe Antwortraten sowie Wirtschaftlichkeit gekennzeichnet ist. Einen Überblick zu allgemeinen Vor- und Nachteilen unterschiedlicher Erhebungsformen gibt Tab. 6.1.

Für die Befragung kam ein computergestützter, standardisierter Fragebogen zum Einsatz (s. Abschnitt A.1 des Anhangs). Ein im Vorfeld durchgeführter zweistufiger Pre-Test gewährleistete, dass einerseits die einzelnen Fragen inhaltlich verständlich, konsistent und fehlerfrei waren und dass andererseits der Begrüßungstext die relevanten Inhalte in angemessener Zeit vermittelte und dabei zugleich zur Teilnahme am Interview motivierte.

Die Befragung fand in angemessenem zeitlichem Abstand von der jährlichen Rechnungstellung der Stromanbieter statt, so dass die Befragungsergebnisse nicht durch ein temporär höheres Involvement verzerrt wurden. Zur Grundgesamtheit zählten alle aktuellen und ehemaligen Privatkunden zweier regionaler Stromanbieter (Anbieter A und B). Aus den Zählerdatenbanken beider Anbieter wurden je 2500 Privathaushalte per Zufallsauswahl gezogen, wobei diejenigen Haushalte übergewichtet eingingen, die den Stromanbieter gewechselt hatten, also ehemalige Kunden sind. Ausgehend von diesen zufällig ausgewählten Haushalten ergaben sich 423 statistisch auswertbare Interviews für die Region A, 510 Interviews für die Region B. Die Teilnahmequote beträgt damit 16,9\% für die Region A und 20,4\% für die Region B. Nach einer Eliminierung von Ausreißern und Datensätzen mit offensichtlichen Widersprüchen konnten letztendlich 902 Datensätze der weiteren Analyse zugeführt werden.

Von den verbliebenen 902 Befragten hatten 80 bereits mindestens einmal den Anbieter gewechselt. Dies entspricht einer Quote von 8,9\%. Vergleicht man diesen Wert mit dem 


\begin{tabular}{lccc}
\hline Kriterium & $\begin{array}{c}\text { persönliche } \\
\text { Befragung }\end{array}$ & $\begin{array}{c}\text { telefonische } \\
\text { Befragung }\end{array}$ & $\begin{array}{c}\text { schriftliche } \\
\text { Befragung }\end{array}$ \\
\hline Antwortrate & hoch & hoch & eher niedrig \\
Flexibilität bei der Erhebung & sehr hoch & hoch & gering \\
Erhebbare Datenmenge & sehr groß & eher gering & mittel \\
Kosten pro Erhebungsfall & hoch & gering & gering \\
Zeitbedarf für die Erhebung & groß & mittel & eher groß \\
Externe Validität & sehr hoch & hoch & mittel \\
Interaktionsmöglichkeit & sehr groß & groß & gering \\
Durchführungsprobleme & zahlreich & gering & sehr gering \\
$\begin{array}{l}\text { Eignung bei hoher Komplexität } \\
\text { der Befragung }\end{array}$ & sehr gut & gut & gering \\
\hline
\end{tabular}

Tabelle 6.1: Übersicht über Vor- und Nachteile verschiedener Befragungsarten (in Anlehnung an Beutin, 2003, S. 129)

Wechsleranteil von 13,9\%, den ZinnBauer (2001, S. 247) aus einer reinen Zufallsstichprobe ohne Höhergewichtung der Wechsler gewonnen hat, kann eine Verzerrung dergestalt, dass nur an Strom interessierte Personen an der Befragung teilgenommen hätten, ausgeschlossen werden.

Demographisch setzen sich die Befragten wie folgt zusammen: 50,8\% der Befragten sind weiblich, 49,2\% männlich. 64,0\% leben in Eigentum, 36,0\% in Miete. Abb. 6.1 zeigt den demographischen Aufbau der Stichprobe.

\subsection{Operationalisierung der Konstrukte}

In den folgenden Unterabschnitten werden die Konstrukte, die Teil des in Abschnitt 4.5 vorgestellten Modells sind, operationalisiert. Unterabschnitt 6.3 .1 beschäftigt sich mit der Operationalisierung der Kundenzufriedenheit. Unterabschnitt 6.3 .2 widmet sich der Operationalisierung der Attraktivität der Alternativen. Unterabschnitt 6.3.3 liefert eine Operationalisierung des komplexen Konstrukts Wechselkosten. Mit der Operationalisierung des Konstrukts Involvement befasst sich Unterabschnitt 6.3.4. Schließlich finden die 


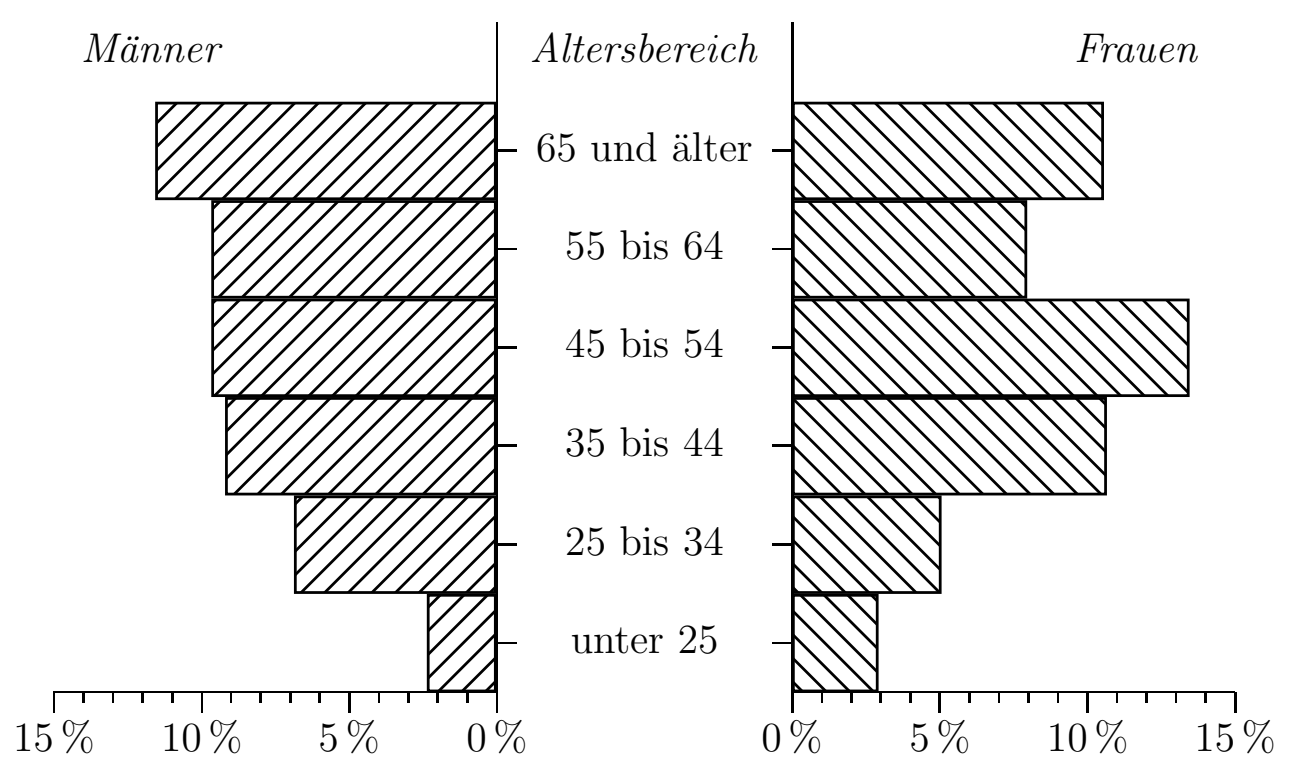

Abbildung 6.1: Zusammensetzung der Stichprobe nach Alter und Geschlecht

endogenen Variablen Wechselabsicht und Wechselvollzug ihre Operationalisierung im Unterabschnitt 6.3.5.

\subsubsection{Operationalisierung der Kundenzufriedenheit}

Aufgrund der Vielzahl von unterschiedlichen Konzeptualisierungen der Kundenzufriedenheit existiert eine nicht minder große Zahl an Ansätzen zu ihrer Messung. Einen Überblick über die verschiedenen Ansätze zur Messung der Kundenzufriedenheit liefert Tab. 6.2. Zunächst ist zwischen subjektiven und objektiven Messverfahren zu differenzieren (Peterson und Wilson, 1992, S. 61).

Subjektive Verfahren fragen die vom Kunden wahrgenommenen, subjektiven Zufriedenheitswerte ab (Homburg und Rudolph, 1998, S. 48). Bei den subjektiven Verfahren lässt sich zwischen ereignisorientierten und merkmalsorientierten Ansätzen unterscheiden (Stauss und Hentschel, 1992, S. 115-117). Ereignisorientierte Ansätze basieren auf der Annahme, dass die vom Kunden empfundene Zufriedenheit auf die Wahrnehmung und psychische Verarbeitung von einzelnen konkreten Ereignissen während des Konsumprozesses zurückgeht. Von den ereignisorientierten Ansätzen zur Messung der Kundenzufriedenheit kommt der Critical Incident Technique eine besondere Bedeutung zu. Hierbei werden Kunden über die mit einem Produkt gemachten Erfahrungen befragt. Ziel ist es, kritische Ereignisse im Rahmen der Anbieter-Kunde-Interaktion zu identifizieren (vgl. 
Ansätze zur Erfassung von Kundenzufriedenheitsurteilen

\begin{tabular}{|c|c|c|c|}
\hline \multirow{3}{*}{$\begin{array}{l}\text { objektive } \\
\text { Verfahren }\end{array}$} & \multicolumn{3}{|c|}{ subjektive Verfahren } \\
\hline & \multicolumn{2}{|c|}{ merkmalsgestützte Verfahren } & \multirow{2}{*}{$\begin{array}{l}\text { ereignis- } \\
\text { orientierte } \\
\text { Verfahren }\end{array}$} \\
\hline & $\begin{array}{l}\text { implizite } \\
\text { Methoden }\end{array}$ & $\begin{array}{l}\text { explizite } \\
\text { Methoden }\end{array}$ & \\
\hline $\begin{array}{l}\text { - Umsatz } \\
\text { - Marktanteil } \\
\text { - Wechselrate } \\
\text { - Wiederkaufrate }\end{array}$ & $\begin{array}{l}\text { - Analyse der } \\
\text { Beschwerden } \\
\text { - Ermittlung der } \\
\text { Leistungsmängel } \\
\text { - Befragung von } \\
\text { Verkäufern und } \\
\text { Absatzmittlern }\end{array}$ & $\begin{array}{l}\text { - Messung des } \\
\text { Erfüllungsgrades } \\
\text { - Messung der } \\
\text { Globalzufrie- } \\
\text { denheit } \\
\text { - Messung der } \\
\text { Dimensionen von } \\
\text { Zufriedenheit } \\
\text { mit Multiattri- } \\
\text { butivmodellen }\end{array}$ & $\begin{array}{l}\text { - Methode der } \\
\text { kritischen } \\
\text { Ereignisse }\end{array}$ \\
\hline
\end{tabular}

Tabelle 6.2: Kundenzufriedenheitsmessung (angelehnt an Herrmann, 1998)

Bitner et AL., 1990, S. 73; Bitner et Al., 1989, S. 98; Bitner Et AL., 1985, S. 49). Merkmalsorientierte Ansätze führen Kundenzufriedenheit auf Eigenschaften des Produkts oder der Geschäftsbeziehung zurück. Sie lassen sich durch eine implizite oder eine explizite Messung verfolgen.

Objektive Verfahren werden in geringerem Umfang eingesetzt und als komplementär zu den subjektiven Verfahren angesehen (PETERson und Wilson, 1992, S. 61). Objektive Verfahren ermitteln die gesuchte Größe so, dass eine verzerrte Wahrnehmung durch Personen ausgeschlossen werden kann. Typische objektive Indikatoren für Kundenzufriedenheit sind Absatz- und Umsatzzahlen, der Marktanteil sowie die Wiederkauf- und die Zurückgewinnungsrate.

Über den in dieser Arbeit betrachteten Markt wurden nach dem Kenntnisstand des Autors bislang noch keine Untersuchungen durchgeführt bzw. veröffentlicht, die einen Kausalzusammenhang zwischen der Kundenzufriedenheit und objektiven Messgrößen wie Umsatz oder Marktanteil nahelegen. Da bisher erst ein Bruchteil der Konsumenten den Anbieter gewechselt hat, hängen bei der Versorgung von Konsumenten Umsatz und Marktanteil offensichtlich eher von der Einwohnerzahl des ehemals monopolistisch belieferten Gebiets als von der Kundenzufriedenheit ab. Hieraus lässt sich schließen, dass objektive Verfahren 
zur Kundenzufriedenheitsmessung im vorliegenden Kontext ungeeignet sind. HomBURG ET AL. (1999, S. 177) empfehlen, insbesondere wenn es darum geht, den Zusammenhang zwischen Kundenzufriedenheit und der Treue von Kunden zu untersuchen, Kundenzufriedenheit als „kognitive und affektive Evaluierung der gesamten Erfahrungen mit einem bestimmten Anbieter und dessen Produkten" (Hervorhebung weggelassen, der Verf.) zu modellieren. Die gleiche Auffassung vertritt Oliver (1999, S. 34), indem er feststellt, dass ,[f]or satisfaction to affect loyalty, frequent or cumulative satisfaction is required so that individual satisfaction episodes become aggregated or blended." Eine Möglichkeit zur Messung bestünde darin, die Teilzufriedenheiten mit den Leistungskomponenten Beschwerdemanagement, Rechnungsstellung, Zusammenarbeit, Kundenzentrum, Dienstleistungen, Energieversorgung und lokales Engagement des Energieversorgers zu erfassen. Es ist jedoch $\mathrm{zu}$ beachten, dass Konsumenten in vielen Produktkategorien keine exakten Produktbeurteilungen bilden und zu keinen expliziten Zufriedenheitsurteilen gelangen (DAY, 1977). Hieraus folgern SWAn und TrAWICK (1979, S. 56), dass wenn man bei der Messung der Kundenzufriedenheit von den Versuchspersonen eine Produktbeurteilung oder das Fällen eines Zufriedenheitsurteils einfordert, dies künstlich einen Evaluationsprozess auslösen und die Befragungsergebnisse verfälschen kann.

Ausgehend von den vorangegangenen Überlegungen empfiehlt sich für die vorliegende Untersuchung eine explizite Messung, d. h. ein merkmalsorientiertes, subjektives Messverfahren. Da im betrachteten Kontext die Erfassung der kumulativen Zufriedenheit am vielversprechendsten ist, soll das bewertete Merkmal der Stromanbieter selbst sein. Es handelt sich somit beim Konstrukt der Kundenzufriedenheit um die von den Versuchspersonen bewertete eigene Zufriedenheit mit dem aktuellen bzw. dem ursprünglichen Anbieter. Skalen, die zur Erfassung kumulativer Kundenzufriedenheit dienen, wurden insbesondere für die nationalen und internatonalen Kundenzufriedenheitsindizes entwickelt. Die für die vorliegende Untersuchung gewählte Operationalisierung des Konstrukts Kundenzufriedenheit entspricht der beim European Customer Satisfaction Index verwendeten Operationalisierung (ChATELIN ET AL., 2002, S. 4). Die Kundenzufriedenheit äußert sich demnach in der Gesamtzufriedenheit, dem Erfüllungsgrad der Erwartungen sowie dem Vergleich mit einem idealen Anbieter. Es handelt sich hierbei um eine Erhebung der Urteile der Kunden auf globaler Ebene (Homburg und Werner, 1998, S. 133). Das resultierende Messmodell ist in Abb. 6.2 dargestellt. Die Antwortskala für diese drei Items besitzt fünf Antwortmöglichkeiten mit den Polen „ganz und gar unzufrieden“ und „voll und ganz zufrieden".

Für Versuchspersonen, die ihren Anbieter gewechselt haben, ist sowohl die Zufriedenheit mit ihrem alten Anbieter als auch die Zufriedenheit mit ihrem neuen Anbieter zu 


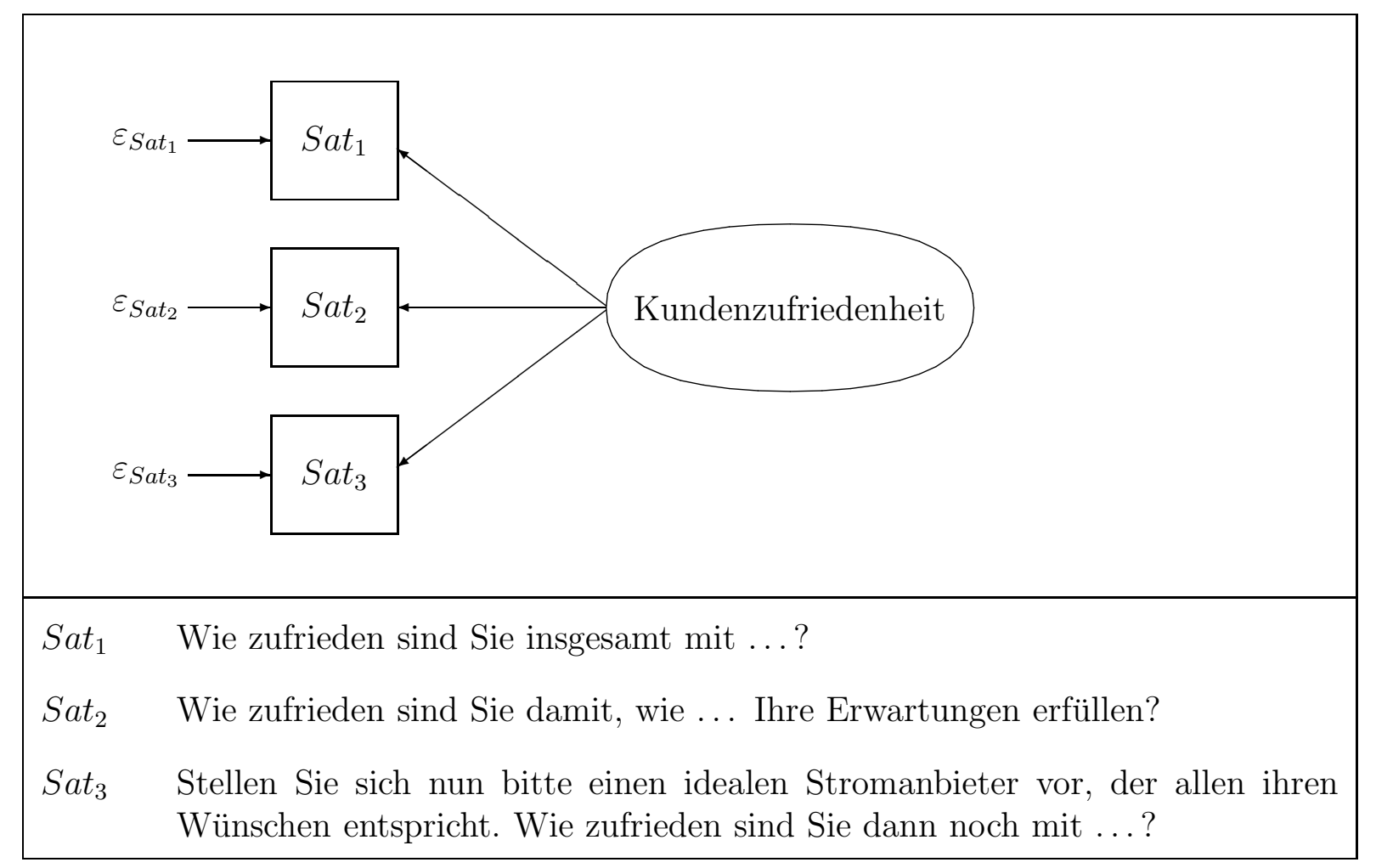

Abbildung 6.2: Messmodell der Kundenzufriedenheit

erheben. Sie mussten deswegen sechs Fragen beantworten: Drei Fragen bzgl. ihrer Zufriedenheit mit dem alten Anbieter $\left(S a t_{1}^{a l t}, S a t_{2}^{a l t}, S a t_{3}^{a l t}\right)$ und drei Fragen bzgl. ihrer Zufriedenheit mit dem neuen Anbieter $\left(S_{1} t_{1}^{\text {neu }}, S_{a} t_{2}^{n e u}, S a t_{3}^{n e u}\right)$. Diejenigen Versuchspersonen, die ihren Anbieter nicht gewechselt haben, können lediglich die Zufriedenheit mit einem Anbieter bewerten. Für sie gilt daher $S a t_{h}^{a l t}=S a t_{h}^{n e u} \forall h$. Ihnen wurden deshalb nur drei Zufriedenheitsfragen gestellt.

\subsubsection{Operationalisierung der Attraktivität der Alternativen}

Analog zur Kundenzufriedenheit lässt sich auch die Attraktivität der Alternativen sowohl durch subjektive als auch durch objektive Verfahren erfassen. Da beide spezifische Vorund Nachteile haben, muss man bei der Operationalisierung eine Abwägung vornehmen.

Objektive Verfahren besitzen zwei große Vorteile: Da die Attraktivität eines Angebots eine unmittelbare Konsequenz des Marketings und seiner Instrumente ist, vermögen objektive Verfahren zum einen leichter die Wirksamkeit bestimmter Marketingmaßnahmen aufzuzeigen. Die Ableitung von Handlungsempfehlungen vereinfacht sich. Zum anderen sind objektive Größen mitunter nicht messfehlerbehaftet. Als Nachteil muss bei objektiven Verfahren zur Messung der Attraktivität der Alternativen die relative Ferne beider 
Konstrukte in Kauf genommen werden. Da Zwischenphasen wie bspw. die Perzeption oder die Verarbeitung der Marketinginstrumente durch Konsumenten nicht untersucht werden, dürfte der Erklärungsbeitrag der objektiv gemessenen Attraktivität der Alternativen innerhalb eines Konsumentenverhaltensmodells tendenziell geringer sein als bei einer subjektiven Messung.

Subjektive Verfahren spielen ihren Vorteil aus, wenn der Erklärungsgrad anderer subjektiv gemessener latenter Variablen maximiert werden soll. Sie messen die gespeicherten Kognitionen und sind damit näher an der Bildung von Absichten. Einerseits ist zu erwarten, dass die subjektiv gemessene Attraktivität der Alternativen in größerem Umfang zur Erklärung von Konsumentenverhalten beiträgt. Andererseits lässt sich die Varianz in der subjektiv gemessenen Attraktivität der Alternativen nur noch teilweise auf die Marketinginstrumente der untersuchten Anbieter zurückführen. Vielmehr spiegelt sie das bei der Perzeption, Verarbeitung und Speicherung von Information hinzugekommene Rauschen wider. Die Erkenntnisse von Wathne ET AL. (2001, S. 55) unterstreichen diesen Sachverhalt: WATHNE ET AL. entdecken einen Zusammenhang zwischen der Wahrnehmung der Attraktivität der Alternativen und dem Ergebnis aus den Interaktionen mit dem derzeitigen Anbieter. Je besser sich die Interaktion mit diesem für eine Person gestaltet, desto weniger attraktiv werden der Person andere Anbieter erscheinen.

Die in der Literatur verwendeten Messinstrumente für die Attraktivität der Alternativen sind überwiegend den subjektiven Verfahren zuzuordnen. Abgesehen von dieser Gemeinsamkeit unterscheiden sie sich stark, wie sich am Beispiel von drei Operationalisierungen veranschaulichen lässt: Jones ET AL. (2000, S. 271) erfassen die Einschätzung von Kunden über die Anzahl möglicher Alternativen. PAtterson und Smith (2003, S. 118) beschränken sich bei der Befragung ihrer Versuchspersonen auf die Anmutung von Ähnlichkeit zwischen allen Anbietern. KIM ET AL. (2004) erfragen Ruf, Image und Dienstleistungsqualität eines Ersatzanbieters und fassen diese Indikatoren zu einem Faktor Attraktivität der Alternativen zusammen. Keine der Operationalisierungen zielt auf die Attraktivität der besten Alternative ab.

In Anbetracht der Vorteile von objektiven Messverfahren und des Umstands, dass auf keine geeigneten subjektiven Messverfahren zurückgegriffen werden kann, kommt in der vorliegenden Arbeit eine objektive Messung zum Einsatz. Gemäß der vorgenommenen Konzeptualisierung entspricht die Attraktivität der Alternativen der Ersparnis, die sich durch den Wechsel zum günstigsten verfügbaren Konkurrenzanbieter erzielen lässt. Die mögliche Ersparnis für einen Haushalt setzt sich aus zwei Elementen zusammen: dem Preisniveau des aktuellen Anbieters im Vergleich zum Preisniveau der attraktivsten Alternative und dem Stromverbrauch des Haushalts. Abb. 6.3 zeigt schematisch die mögliche 


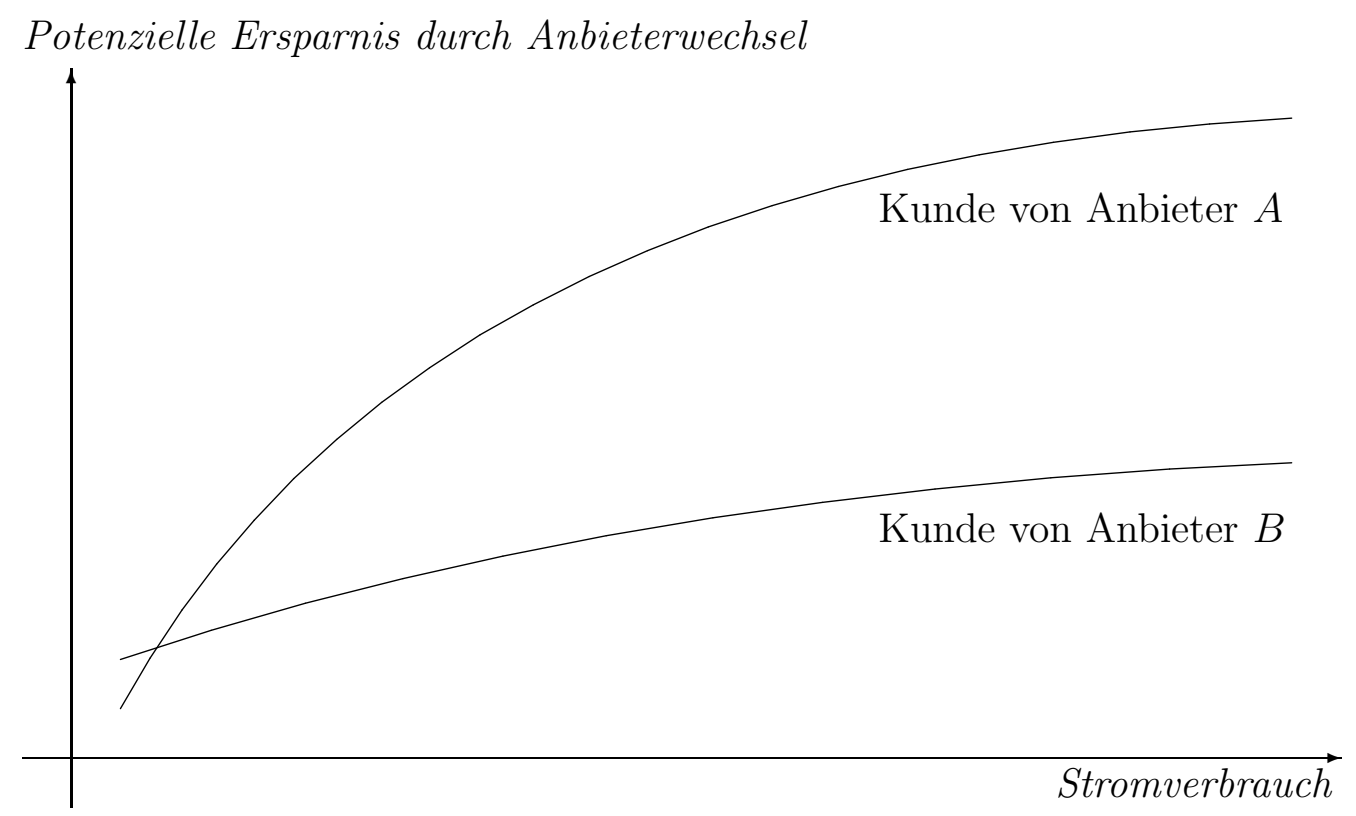

Abbildung 6.3: Potenzielle Ersparnis durch einen Anbieterwechsel in Abhängigkeit vom Stromverbrauch

relative Ersparnis, die Kunden durch einen Anbieterwechsel erzielen können, in Abhängigkeit vom Stromverbauch und vom aktuellen Anbieter.

Aus der Abbildung wird deutlich, dass für Kunden des Anbieters $A$, der eine Hochpreisstrategie verfolgt, ein Anbieterwechsel attraktiver ist als für Kunden des Anbieters $B$, der eine Niedrigpreisstrategie anwendet. Weiterhin ist ersichtlich, dass die relative Ersparnis (Geldeinheiten pro Stromeinheit), die sich durch einen Anbieterwechsel erzielen lässt, mit zunehmendem Stromverbrauch steigt. Als Konsequenz ist auch die absolute Ersparnis für einen Kunden umso höher, je mehr Strom er verbraucht.

Ausgehend von diesen Überlegungen werden zwei Indikatoren verwendet, um die Attraktivität der Alternativen objektiv zu messen: erstens der Anbieter, bei dem sich die einzelnen Versuchspersonen befinden ( $A$ oder $B$ ), und zweitens die Haushaltsgröße als Substitut für den Stromverbrauch. Beide Faktoren, der ursprüngliche Anbieter (A oder B) und die Haushaltsgröße, gehen formativ in einen Index der Attraktivität des Anbieterwechsels ein. Abb. 6.4 zeigt das resultierende Messmodell.

\subsubsection{Operationalisierung der Wechselkosten}

Durchsucht man die marketingwissenschaftliche Literatur, stößt man auf zwei Methoden zur Erhebung der Wechselkosten: Die erste Methode besteht in Expertenurteilen über die 


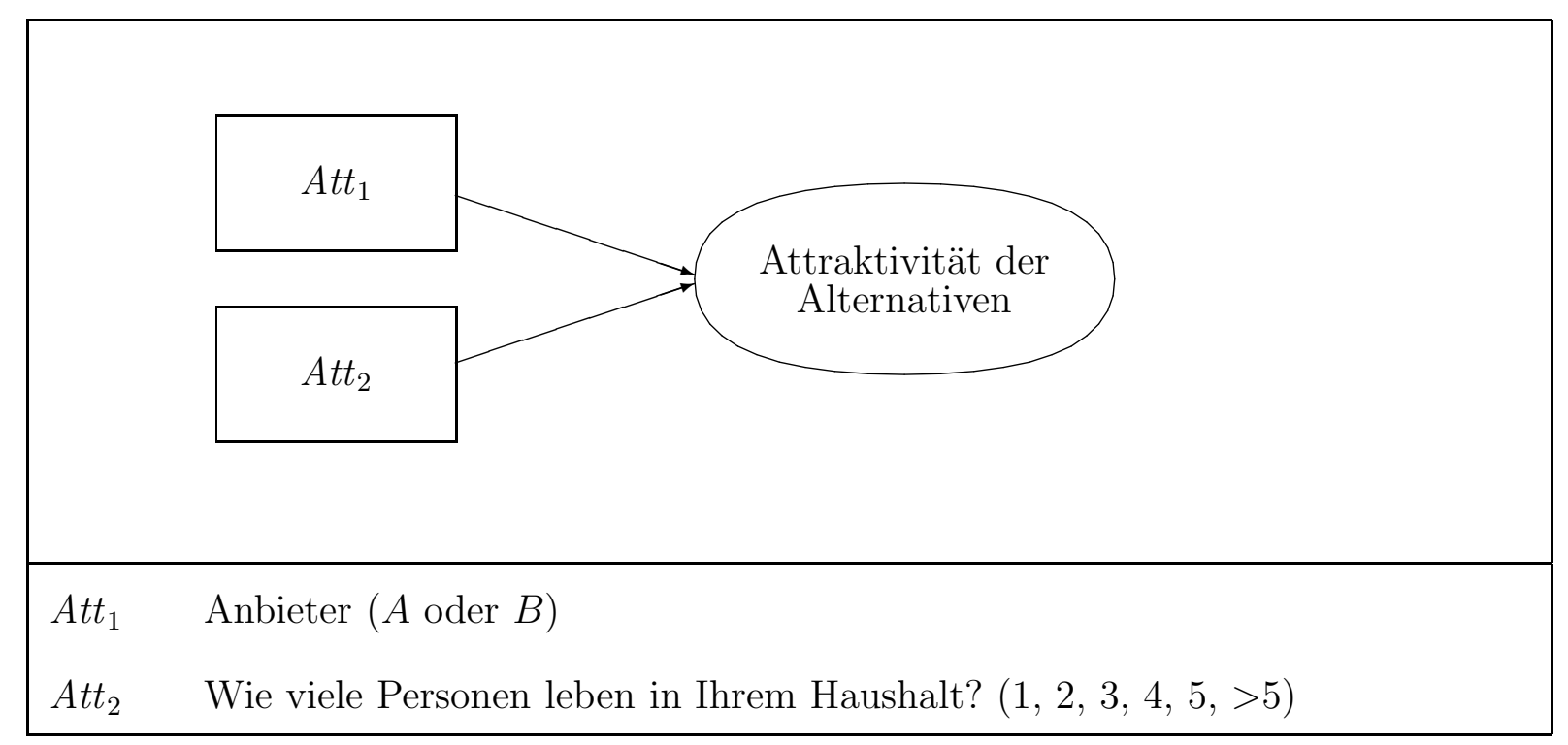

Abbildung 6.4: Index der Attraktivität der Alternativen

in einer Branche vorhandenen Wechselkosten (vgl. z. B. Anderson, 1994, S. 25). Laut dem dieser Arbeit zugrunde liegenden Verständnis von Wechselkosten kann es sich bei den auf diese Weise gemessenen Wechselkosten nur um Wechselbarrieren handeln. Nicht zuletzt aufgrund der fehlenden Variation unter den Kunden einer Branche eignet sich diese Methode kaum zur Modellierung des Konsumentenverhaltens. Die zweite Methode ist die direkte Befragung von Versuchspersonen bezüglich ihrer wahrgenommenen Wechselkosten. Diese Methode findet hier Anwendung.

In vielen bisherigen Arbeiten zum Einfluss der Wechselkosten in Dienstleistungsbeziehungen wurden die Wechselkosten unidimensional konzeptualisiert (vgl. beispielhaft MAUTE und Forrester, 1993). Frühere konzeptionelle Arbeiten zu Wechselkosten (bspw. KLEMPERER, 1987b; GuILTinan, 1989) legten jedoch bereits dar, dass es mehrere Dimensionen von Wechselkosten gibt. Nur ein mehrdimensionaler Forschungsansatz ermöglicht es, wichtige theoretische und praktische Unterscheidungen über Dimensionen hinweg aufzudecken (Jones ET AL., 2002, S. 441). Um das Konstrukt der Wechselkosten in seiner gesamten Breite zu erfassen, verfolgt die vorliegende Arbeit einen mehrdimensionalen Ansatz.

Da sich die wahrgenommenen Wechselkosten aus verschiedenen Komponenten zusammensetzen, empfiehlt sich die Konzeptualisierung als Konstrukt zweiter Ordnung im Sinne von JARVIS ET AL. (2003). Dabei stellt sich die Frage, welche Messmodellart (reflektiv oder formativ) dem Konstrukt Wechselkosten zuteil werden soll. Zur Entscheidungsfindung lässt sich eine einfache Frage formulieren: Wenn man verschiedene Kosten misst, reflektieren diese Messungen dann die Gesamtkosten oder setzen sich die Gesamtkosten aus Teilkosten zusammen? Die Kostenrechnung lehrt letzteres: Verschiedene Teilkosten wie 
z. B. Kostenarten werden zu den Gesamtkosten aufsummiert. Für das Messmodell heißt dies, dass eine formative Operationalisierung angebracht ist.

Im Rahmen der Hypothesenentwicklung wurden als maßgebliche Komponenten der Wechselkosten der direkte Aufwand eines Wechsels, die Such- und Informationskosten sowie das wahrgenommene Risiko identifiziert. Offensichtlich führen Änderungen in den Komponenten zu Änderungen der gesamten Wechselkosten, während eine Änderung der Wechselkosten nicht zwingend mit der Änderung aller Komponenten einhergeht. Auch die übrigen Fragen aus dem vorgestellten Fragenkatalog von JARVIS ET AL. (2003) legen eine formative Operationalisierung nahe. Analog bestehen auch die drei Wechselkomponenten „direkter Aufwand eines Wechsels“, „Such- und Informationskosten“ sowie „wahrgenommenes Risiko" aus Teilkosten, die mittels Indikatoren abgefragt werden können. Aus obiger Analyse ergibt sich, dass für das Konstrukt Wechselkosten insgesamt eine formative Operationalisierung zum Tragen kommen muss. Es handelt sich also um eine Spezifikation eines Konstrukts zweiter Ordnung vom Typ IV, d. h. sowohl die einzelnen Komponenten als auch das Konstrukt zweiter Ordnung werden formativ operationalisiert.

Zunächst werden die drei Wechselkostenkomponenten als alleinstehende Konstrukte mit jeweils zwei Indikatoren operationalisiert:

Direkter Aufwand eines Wechsels: In Anlehnung an die Skala von PING (1993) werden die Versuchpersonen dazu befragt, für wie aufwendig und wie mühevoll sie einen Anbieterwechsel halten. Abb. 6.5 verbildlicht das formative Messmodell für den direkten Aufwand eines Wechsels.

Such- und Informationskosten: Abb. 6.6 illustriert das formative Messmodell für die Wechselkostenkomponente „Such- und Informationskosten“. Der erste Indikator $\left(W k s_{1}\right)$ erfragt die Menge an Arbeit, die notwendig ist, sich über Stromtarife zu

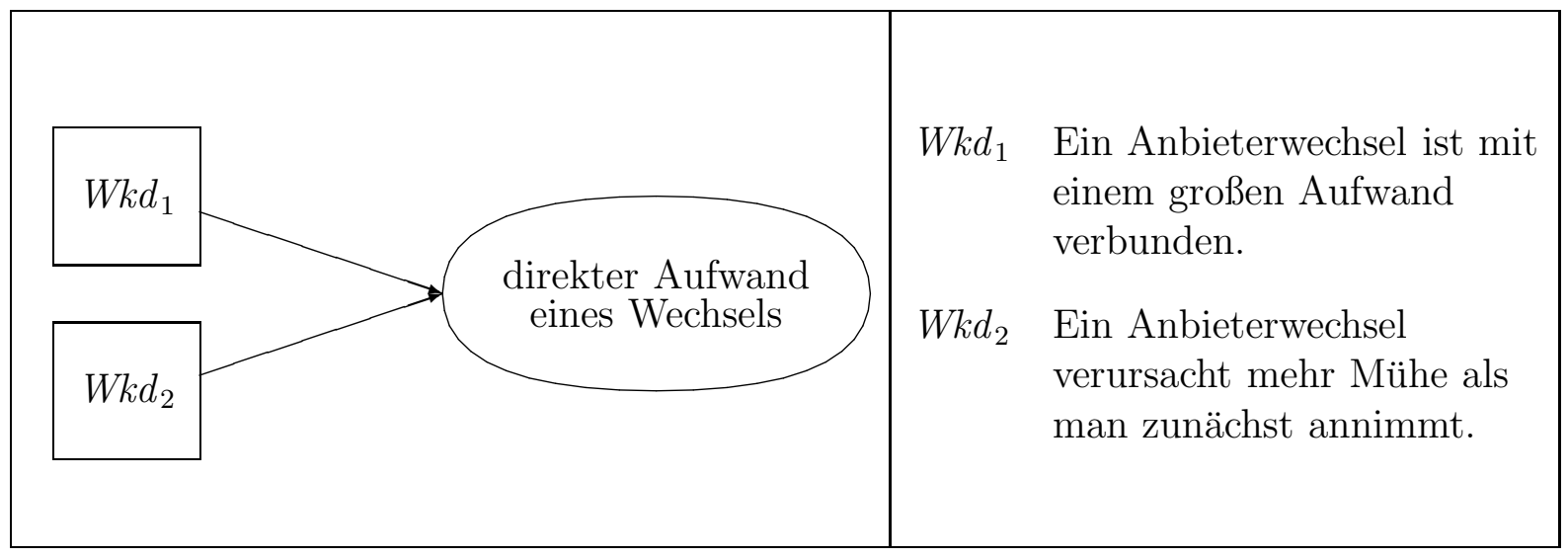

Abbildung 6.5: Messmodell des direkten Aufwands eines Wechsels 


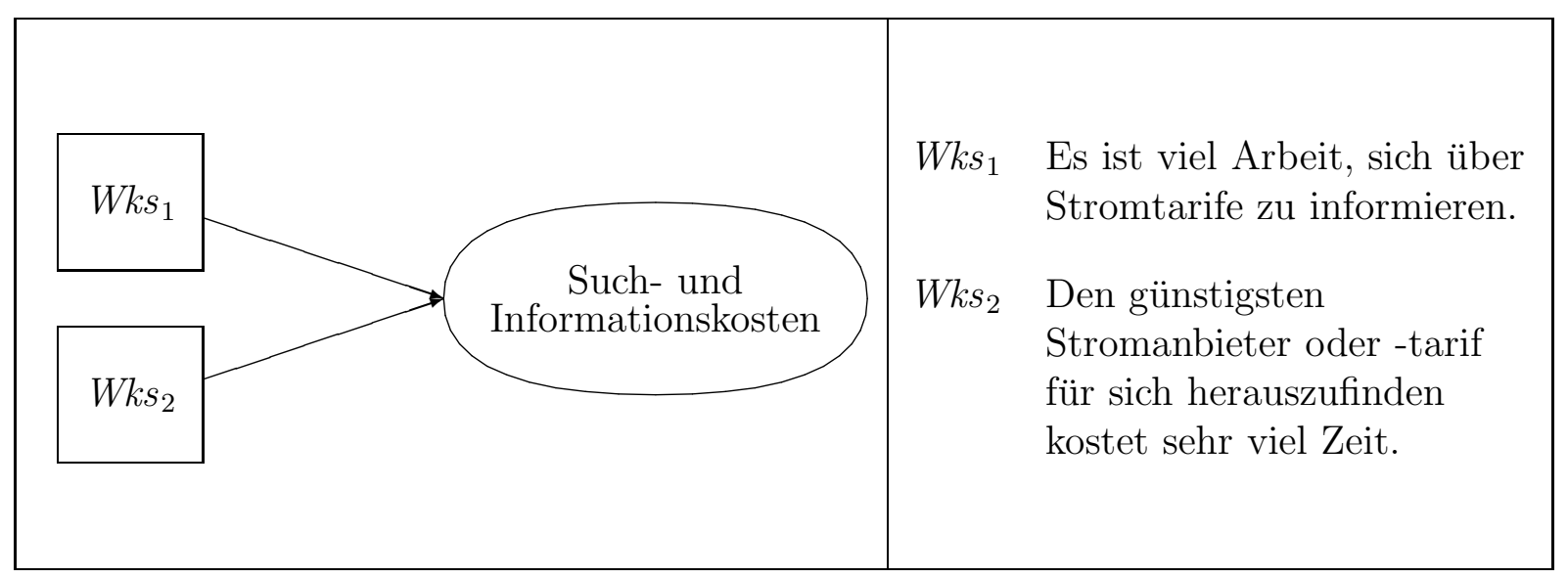

Abbildung 6.6: Messmodell der Such- und Informationskosten

informieren. Der zweite Indikator $\left(W k s_{2}\right)$ fragt nach dem zeitlichen Aufwand für die Suche nach dem günstigsten Stromanbieter oder -tarif.

Wahrgenommenes Risiko: Die Operationalisierung des wahrgenommenen Risikos setzt an den beiden Komponenten an, die Cox (1967b) als maßgeblich für die Höhe des Risiko ansieht: der Schwere der negativen Konsequenzen sowie der Wahrscheinlichkeit des Auftretens der negativen Konsequenzen. Die Versuchspersonen werden gefragt, wie viel man bei der Wahl des falschen Stromanbieters zu verlieren hat und wie leicht man sich bei der Auswahl eines neuen Stromversorgers irren kann. Abb. 6.7 zeigt das resultierende Messmodell.

Alle Indikatoren der unterschiedlichen Wechselkostenkomponenten besitzen fünf Antwortmöglichkeiten mit den Polen „stimme ganz und gar nicht zu“ und „stimme voll und ganz zu“ sowie der expliziten Mitte „teils teils“.

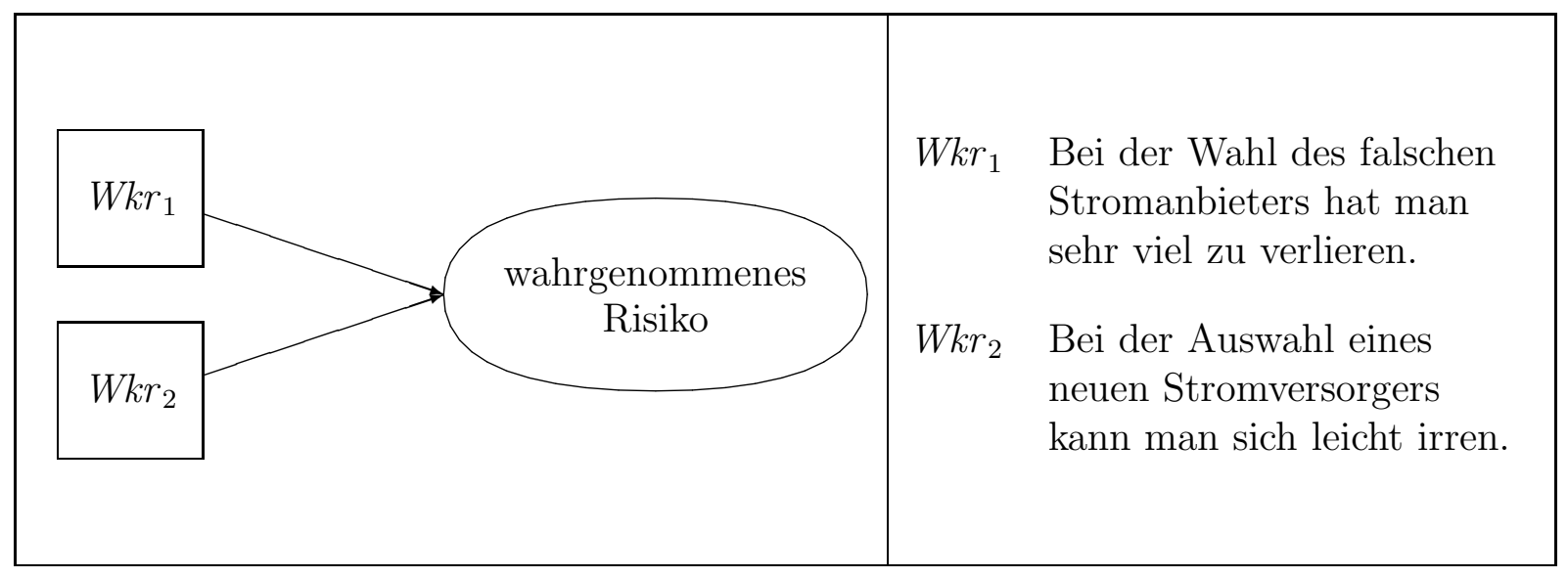

Abbildung 6.7: Messmodell des wahrgenommenen Risikos 


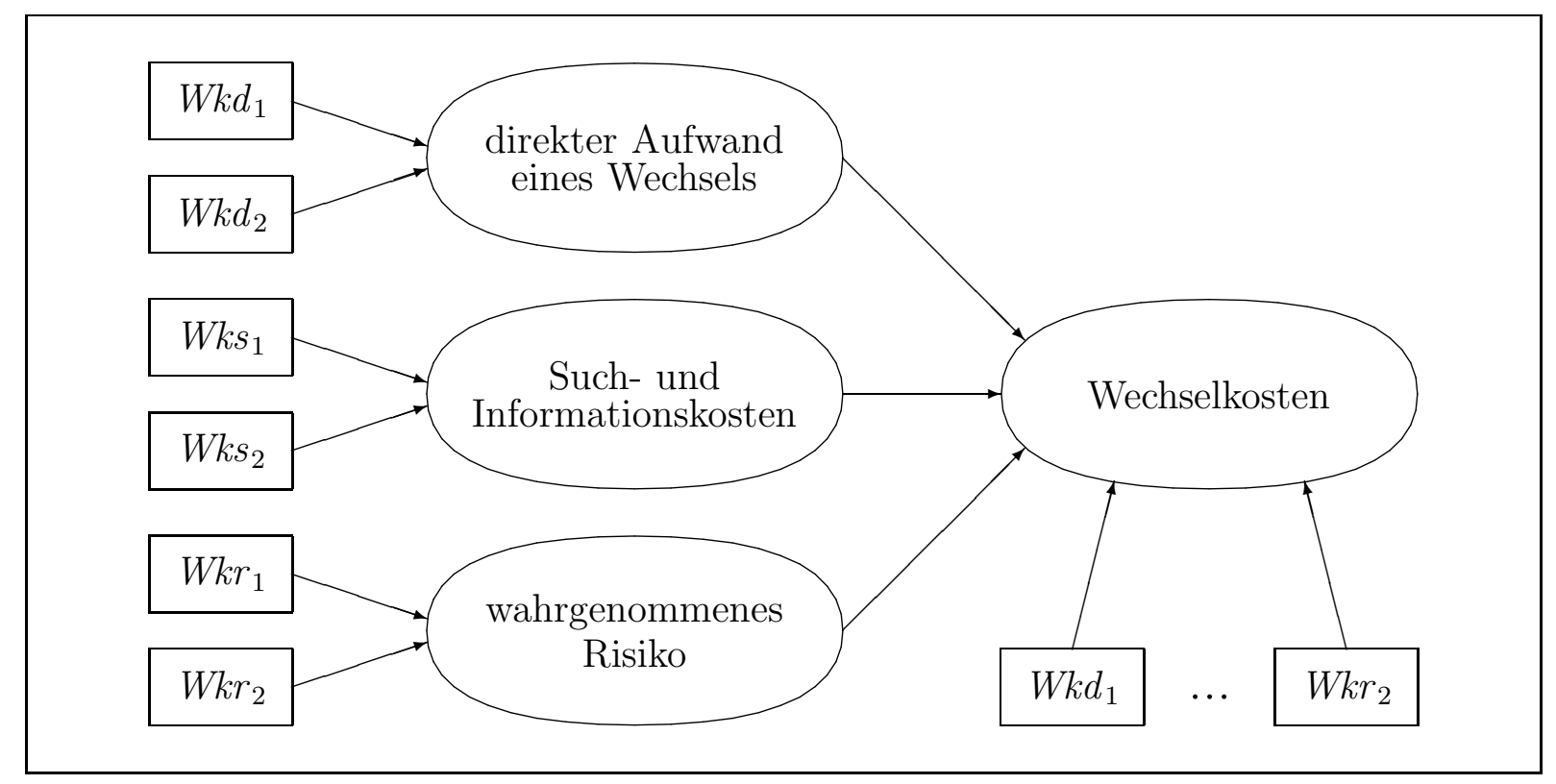

Abbildung 6.8: Operationalisierung der Wechselkosten als Konstrukt zweiter Ordnung

Das Konstrukt Wechselkosten setzt sich aus den drei Wechselkostenkomponenten zusammen. Um es durch den PLS-Algorithmus schätzen zu lassen, werden ihm die sechs Indikatoren der einzelnen Wechselkomponenten zugewiesen. Abb. 6.8 zeigt die hierarchische Struktur des komplexen Konstrukts Wechselkosten.

\subsubsection{Operationalisierung des Involvements}

In der Literatur findet man verschiedene Operationalisierungen des Involvementkonstrukts - sowohl in Bezug auf die Form des Messmodells als auch auf die inhaltliche Ausgestaltung. Während einige Forscher eine unidimensionale reflektive Operationalisierung favorisieren, bevorzugen ZAICHKOWsky und Sood (1988, S. 23f.) ein formatives Messmodell in Form der Summenskala des „Personal Involvement Inventory“. KAPfereR und LAURENT (1993, S. 347) widersprechen beiden Auffassungen vehement und plädieren für eine mehrdimensionale Konzeptualisierung und Operationalisierung des InvolvementKonstrukts.

Beim Involvementkonstrukt, wie es in der vorliegenden Arbeit operationalisiert werden soll, handelt es sich genau genommen um das Produktinvolvement, das sich vor allem im Interesse am Produkt und der Beschäftigung mit dem Produkt äußert. Anders als bei vielen anderen Produkten ist die Wichtigkeit des Produkts kein Indikator für das Involvement, da die Wichtigkeit von Strom allgemein als recht hoch eingeschätzt wird. Die gewählten Indikatoren sind angelehnt an die Operationalisierung von BAKAY (2003). Abb. 6.9 zeigt das Messmodell des Involvements. 


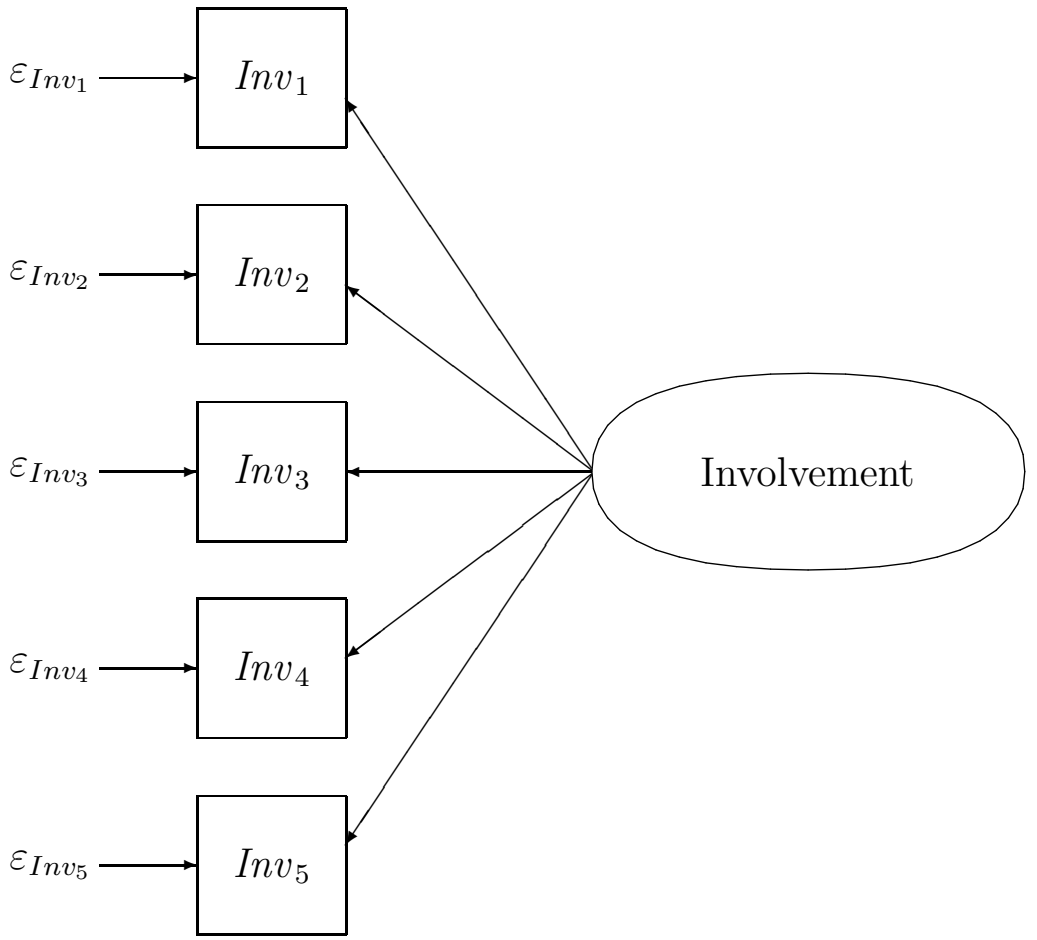

$\operatorname{Inv}_{1} \quad$ Ich interessiere mich für die verschiedenen Angebote im Strommarkt.

$I_{n} v_{2} \quad$ Seitdem man seinen Stromanbieter frei wählen kann, achte ich auch verstärkt auf die Leistungen meines eigenen Stromanbieters.

$\mathrm{Inv}_{3} \quad$ Die neuen Anbieter im Strommarkt wecken meine Aufmerksamkeit.

$\operatorname{Inv}_{4} \quad$ Ich interessiere mich für Preisvergleiche zwischen verschiedenen Stromanbietern.

$I_{n v_{5}} \quad$ Sollte mich jemand zu Stromanbietern um Rat fragen, so könnte ich ihm Auskunft geben.

Abbildung 6.9: Messmodell des Involvements

Auch beim Involvement besitzt die Antwortskala für alle Items fünf Antwortmöglichkeiten mit den Polen „stimme ganz und gar nicht zu“ und „stimme voll und ganz zu“ sowie der expliziten Mitte „teils teils“.

\subsubsection{Operationalisierung der endogenen Variablen}

Die Operationalisierung der Wechselabsicht orientierte sich an der Skala von OLIVER und SwAn (1989). Da auch bei der Angabe der Wechselabsicht mit Messfehlern zu rechnen ist, empfiehlt sich auch hier eine reflektive Operationalisierung. Abb. 6.10 zeigt das gewählte Messmodell. 


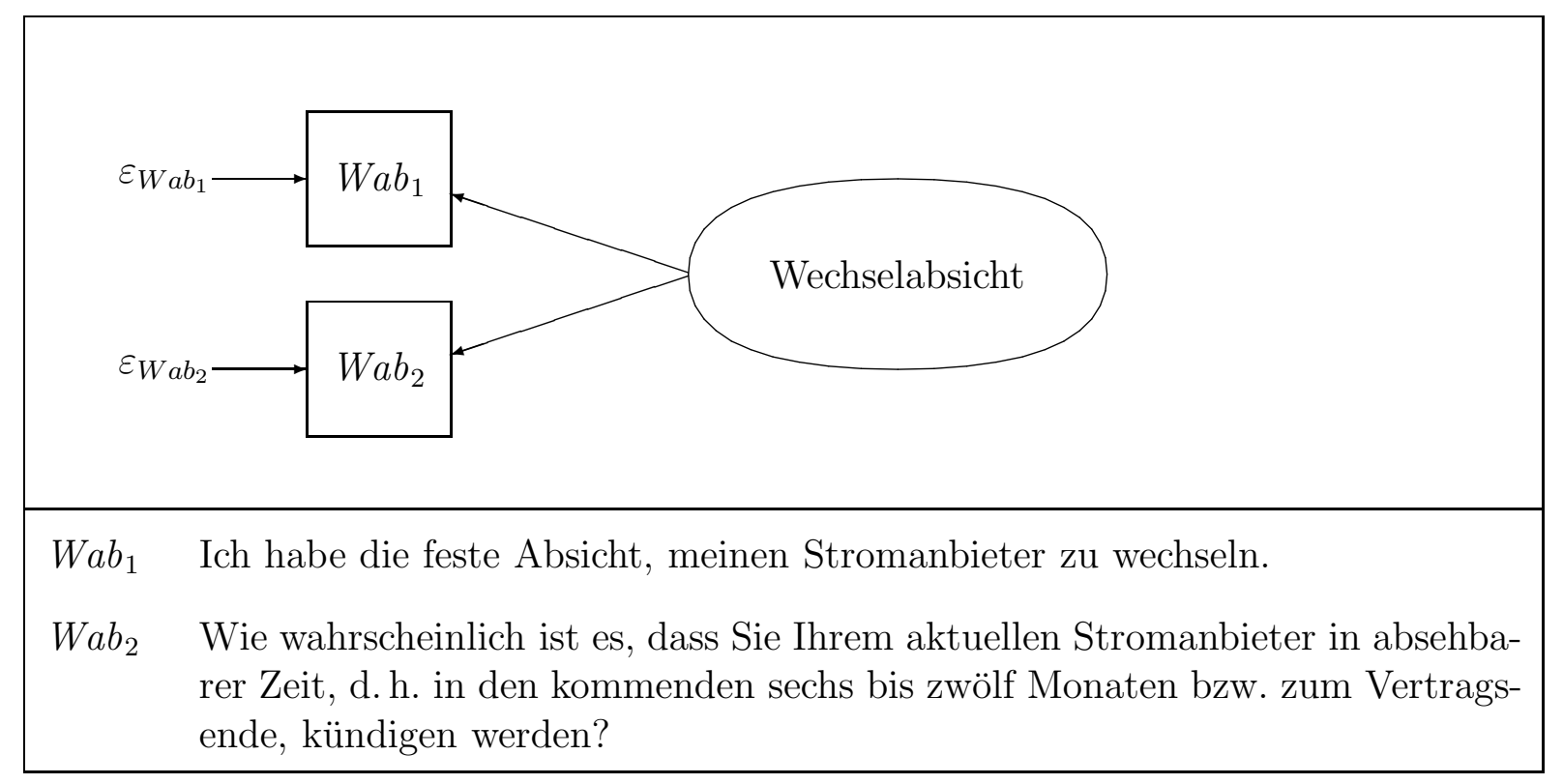

Abbildung 6.10: Messmodell der Wechselabsicht

Für den ersten Indikator $\left(W a b_{01}\right)$ kam erneut die Antwortskala aus fünf Antwortmöglichkeiten mit den Polen „stimme ganz und gar nicht zu“ und „stimme voll und ganz zu“ sowie der expliziten Mitte „,teils teils“ zum Einsatz. Für die Frage nach der Wechselwahrscheinlichkeit $\left(W a b_{02}\right)$ wurde hingegen die sechsstufige Wahrscheinlichkeitsskala von KoTLER und Bliemel (2001, S. 254) mit den folgenden Stufen verwendet:

$\begin{array}{ll}0,0 & \text { unter keinen Umständen } \\ 0,2 & \text { sehr unwahrscheinlich } \\ 0,4 & \text { vielleicht } \\ 0,6 & \text { gut möglich } \\ 0,8 & \text { sehr wahrscheinlich } \\ 1,0 & \text { sicher }\end{array}$

Die Operationalisierung des Wechselvollzugs ist relativ einfach, da es sich um keine wirklich „latente“ Variable handelt. Die Messung erfolgte mit einer einfachen Frage nach einem bereits vollzogenen Wechsel. Als Antwortmöglichkeiten standen den Versuchspersonen „Ja“, „Nein" und „Weiß nicht" zur Auswahl.

\subsection{Beurteilung des Messmodells}

Bevor die aufgestellten Hypothesen getestet werden, ist zunächst die Qualität des Messmodells zu prüfen. Im Zentrum steht dabei die Frage, ob die einzelnen Konstrukte reliabel und valide gemessen wurden. 
Die ersten beiden Unterabschnitte betrachten die Indikatorkoeffizienten: Unterabschnitt 6.4.1 analysiert die Indikatorladungen, Unterabschnitt 6.4.2 informiert bei den formativen Konstrukten über die Indikatorgewichte. Der dritte Unterabschnitt (6.4.3) beschäftigt sich mit der Messmodellreliabilität, und der vierte Unterabschnitt (6.4.4) geht auf die Messmodellvalidität ein.

\subsubsection{Beurteilung der Indikatorladungen}

Tab. 6.3 gibt Aufschluss über die Ladungen der standardisierten reflektiven Indikatoren. Die erste Wertespalte von Tab. 6.3 zeigt die Werte der Indikatorladungen $\lambda$. Abgesehen von der als fehlerfrei angenommenen Variable $W e c$, die eine Ladung von eins aufweist, zeigen auch die Indikatoren von Kundenzufriedenheit und Wechselabsicht sehr hohe Ladungen. Etwas geringer fallen die meisten Ladungen beim Involvement aus. Die nächste Spalte enthält die Standardabweichungen der Ladungen $s_{\lambda}$. Diese Werte entstammen einem Bootstrapping, bei dem das Modell 200 mal mit je 902 zufällig gezogenen Datensätzen geschätzt wurde. In Relation zur Größe der Ladungen sind die Standardabweichungen sehr gering. Dies spiegelt sich auch in den Konfidenzintervallen wider: Mit 95-prozentiger Wahrscheinlichkeit liegen die Ladungen innerhalb der Grenzen $\lambda_{u}$ und $\lambda_{o}$. Der Signifikanztest liefert wenig Überraschendes: Die Wahrscheinlichkeit, dass eine der Ladungen kleiner oder gleich null ist, erweist sich als äußerst gering.

Eine Besonderheit stellt das Konstrukt Kundenzufriedenheit dar: Konsumenten, die ihren Anbieter gewechselt hatten, gaben Zufriedenheitsurteile sowohl über den ehemaligen $\left(S a t_{h}^{\text {alt }}\right)$ als auch über den neuen Anbieter $\left(S a t_{h}^{n e u}\right)$ ab. Für Konsumenten, die nicht gewechselt hatten, gab es im Prinzip nur einen Zufriedenheitswert $\left(S a t=S a t_{h}^{\text {neu }}=S a t_{h}^{a l t}\right)$, der repliziert wurde.

\subsubsection{Beurteilung der Indikatorgewichte}

Tab. 6.4 gibt Aufschluss über die Gewichte der formativen Konstrukte, also der Attraktivität der Alternativen und der wahrgenommenen Wechselkosten. Die Attraktivität der Alternativen wird analog zur Kundenzufriedenheit zweimal aufgeführt. Die einzelnen Dimensionen der Wechselkosten werden zunächst als eigenständige Konstrukte aufgeführt. Später dienen sie selbst als Indikatoren des Konstrukts zweiter Ordnung.

Die erste Wertespalte von Tab. 6.4 zeigt die Werte der Indikatorgewichte $\pi$. Hierbei fällt auf, wie unterschiedlich die Indikatoren zum Konstrukt Attraktivität der Alternativen beitragen: Während das Gewicht des Anbieter-Indikators $\left(A t t_{1}^{\text {alt }}\right)$ dominiert, wenn es um 


\begin{tabular}{|c|c|c|c|c|c|}
\hline Konstrukt & Indikator & $\lambda$ & $\mathbf{s}_{\lambda}$ & {$\left[\begin{array}{llll}\lambda_{\mathbf{u}} & ; & \lambda_{\mathbf{o}}\end{array}\right]$} & $\mathrm{p}$ \\
\hline \multirow{5}{*}{ Involvement } & $\operatorname{Inv} v_{1}$ & 0,7814 & 0,0189 & {$[0,7441 ; 0,8187]$} & $<0,001$ \\
\hline & $\operatorname{Inv}_{2}$ & 0,5713 & 0,0353 & {$[0,5016 ; 0,6410]$} & $<0,001$ \\
\hline & $\operatorname{Inv}_{3}$ & 0,7824 & 0,0189 & {$[0,7452 ; 0,8196]$} & $<0,001$ \\
\hline & $\operatorname{Inv}_{4}$ & 0,8302 & 0,0152 & {$[0,8003 ; 0,8601]$} & $<0,001$ \\
\hline & $\operatorname{Inv}_{5}$ & 0,6165 & 0,0319 & {$[0,5536 ; 0,6794]$} & $<0,001$ \\
\hline \multirow{6}{*}{ Kundenzufriedenheit } & $S a t_{1}^{a l t}$ & 0,9202 & 0,0445 & {$[0,8324 ; 1,0079]$} & $<0,001$ \\
\hline & $S a t_{2}^{a l t}$ & 0,8993 & 0,0463 & {$[0,8081 ; 0,9906]$} & $<0,001$ \\
\hline & $S a t_{3}^{a l t}$ & 0,9319 & 0,0316 & {$[0,8695 ; 0,9943]$} & $<0,001$ \\
\hline & $S a t_{1}^{n e u}$ & 0,9452 & 0,0178 & {$[0,9100 ; 0,9804]$} & $<0,001$ \\
\hline & $S a t_{2}^{n e u}$ & 0,9420 & 0,0189 & {$[0,9047 ; 0,9793]$} & $<0,001$ \\
\hline & $S a t_{3}^{\text {neu }}$ & 0,8865 & 0,0189 & {$[0,8493 ; 0,9237]$} & $<0,001$ \\
\hline \multirow{2}{*}{ Wechselabsicht } & $W a b_{1}$ & 0,8722 & 0,0212 & {$[0,8304 ; 0,9141]$} & $<0,001$ \\
\hline & $W a b_{2}$ & 0,9284 & 0,0099 & {$[0,9088 ; 0,9480]$} & $<0,001$ \\
\hline Wechselvollzug & $W e c$ & 1,0000 & 0,0000 & {$[1,0000 ; 1,0000]$} & $<0,001$ \\
\hline
\end{tabular}

Tabelle 6.3: Beurteilung der Ladungen der reflektiven Indikatoren: Ladung $\lambda$, Streuung $s_{\lambda}, 95 \%$-Konfidenzintervall $\left[\lambda_{u}, \lambda_{o}\right]$ sowie Irrtumswahrscheinlichkeit $p$, dass $\lambda \leq 0$

den Wechselvollzug geht, erweist sich die Haushaltsgröße $\left(A t t_{2}^{n e u}\right)$ als entscheidende Determinante der Attraktivität der Alternativen, wenn die Wechselabsicht erklärt werden soll.

Die zweite Wertespalte enthält die Standardabweichungen der Gewichte $s_{\pi}$. Sie wurden aus dem im vorhergehenden Unterabschnitt beschriebenen Bootstrapping gewonnen. Die Standardabweichungen der Gewichte sind in der Mehrzahl deutlich größer als die Standardabweichungen der Ladungen. Dies hat natürlich unmittelbare Auswirkungen auf die Konfidenzintervalle $\left[\pi_{u} ; \pi_{o}\right]$. Betrachtet man die Signifikanzen der Indikatorgewichte, stechen die beiden nicht-signifikanten Gewichte der Attraktivität der Alternativen hervor. Dies unterstreicht die Beobachtung aus dem vorigen Absatz über die unterschiedliche Rolle der Indikatoren der Attraktivität der Alternativen.

Ein besonderes Augenmerk verdienen die Gewichte des Konstrukts zweiter Ordnung, der wahrgenommenen Wechselkosten: Alle drei Dimensionen tragen deutlich zum Gesamt- 


\begin{tabular}{|c|c|c|c|c|c|}
\hline Konstrukt & Indikator & $\pi$ & $\mathbf{s}_{\pi}$ & {$\left[\begin{array}{llll}\pi_{\mathbf{u}} & ; & \pi_{\mathbf{o}}\end{array}\right]$} & $p$ \\
\hline \multirow{4}{*}{$\begin{array}{l}\text { Attraktivität der } \\
\text { Alternativen }\end{array}$} & $A t t_{1}^{a l t}$ & 0,9769 & 0,0864 & {$[0,8066 ; 1,1472]$} & $<0,001$ \\
\hline & $A t t_{2}^{a l t}$ & 0,1164 & 0,2527 & {$[-0,3819 ; 0,6148]$} & 0,323 \\
\hline & $A t t_{1}^{\text {neu }}$ & 0,2877 & 0,1844 & {$[-0,0758 ; 0,6512]$} & 0,060 \\
\hline & $A t t_{2}^{\text {neu }}$ & 0,9693 & 0,0875 & {$[0,7967 ; 1,1418]$} & $<0,001$ \\
\hline \multirow{2}{*}{$\begin{array}{l}\text { direkter Aufwand } \\
\text { eines Wechsels ( } D A)\end{array}$} & $W k d_{1}$ & 0,3699 & 0,1210 & {$[0,1352 ; 0,6085]$} & 0,001 \\
\hline & $W k d_{2}$ & 0,7917 & 0,1034 & {$[0,5878 ; 0,9956]$} & $<0,001$ \\
\hline \multirow{2}{*}{$\begin{array}{l}\text { Such- und Informa- } \\
\text { tionskosten }(S K)\end{array}$} & $W k s_{1}$ & 0,4197 & 0,0816 & {$[0,2588 ; 0,5806]$} & $<0,001$ \\
\hline & $W k s_{2}$ & 0,7298 & 0,0677 & {$[0,5963 ; 0,8633]$} & $<0,001$ \\
\hline \multirow{2}{*}{$\begin{array}{l}\text { wahrgenommenes } \\
\text { Risiko (WR) }\end{array}$} & $W k r_{1}$ & 0,6679 & 0,0896 & {$[0,4911 ; 0,8446]$} & $<0,001$ \\
\hline & $W k r_{2}$ & 0,5234 & 0,0970 & {$[0,3321 ; 0,7147]$} & $<0,001$ \\
\hline \multirow{3}{*}{$\begin{array}{l}\text { wahrgenommene } \\
\text { Wechselkosten }\end{array}$} & $D A$ & 0,5064 & 0,0173 & {$[0,4724 ; 0,5405]$} & $<0,001$ \\
\hline & $S K$ & 0,3858 & 0,0152 & {$[0,3558 ; 0,4157]$} & $<0,001$ \\
\hline & $W R$ & 0,3747 & 0,0128 & {$[0,3496 ; 0,3999]$} & $<0,001$ \\
\hline
\end{tabular}

Tabelle 6.4: Beurteilung der Gewichte der formativen Indikatoren: Gewicht $\pi$, Streuung $s_{\pi}, 95 \%$-Konfidenzintervall $\left[\pi_{u}, \pi_{o}\right]$ sowie Irrtumswahrscheinlichkeit $p$, dass $\pi \leq 0$

konstrukt bei. Das größte Gewicht weist mit 0,5064 der direkte Wechselaufwand auf. Aus dem Bootstrapping lässt sich die Information gewinnen, dass die jeweilige Differenz zwischen dem Gewicht des direkten Wechselaufwands und den Gewichten der übrigen beiden Dimensionen signifikant größer als null ist (Irrtumswahrscheinlichkeit $p<0,001$ ), d. h. der direkte Wechselaufwand spielt unter den einzelnen Wechselkostenkomponenten die größte Rolle.

\subsubsection{Beurteilung der Reliabilität}

Dieser Unterabschnitt beschäftigt sich damit, inwieweit die reflektiv operationalisierten Konstrukte reliabel gemessen wurden. Eine analoge Beurteilung formativ operationalisierter Konstrukte ist nicht möglich. Die Überprüfung der Reliabilität setzt an zwei Stellen an: Zunächst erfolgt die Analyse der Reliabilität der einzelnen Indikatoren; im Anschluss wird die Analyse auf die Konstruktebene ausgedehnt. 


\begin{tabular}{|c|c|c|c|c|c|}
\hline Konstrukt & Indikator & $\lambda^{2}$ & $\alpha$ & $\rho_{\mathbf{c}}$ & AVE \\
\hline \multirow{5}{*}{ Involvement } & $\operatorname{Inv} v_{1}$ & 0,6105 & \multirow{5}{*}{0,7701} & \multirow{5}{*}{0,8403} & \multirow{5}{*}{0,5179} \\
\hline & $\operatorname{Inv}_{2}$ & 0,3264 & & & \\
\hline & $\operatorname{Inv}_{3}$ & 0,6121 & & & \\
\hline & $\operatorname{Inv}_{4}$ & 0,6892 & & & \\
\hline & $\operatorname{Inv}_{5}$ & 0,3801 & & & \\
\hline \multirow{6}{*}{ Kundenzufriedenheit } & $S a t_{1}^{a l t}$ & 0,8467 & \multirow{3}{*}{0,8374} & \multirow{3}{*}{0,9060} & \multirow{3}{*}{0,7626} \\
\hline & $S a t_{2}^{a l t}$ & 0,8088 & & & \\
\hline & $S a t_{3}^{a l t}$ & 0,8684 & & & \\
\hline & $S a t_{1}^{\text {neu }}$ & 0,8935 & \multirow{3}{*}{0,8448} & \multirow{3}{*}{0,9143} & \multirow{3}{*}{0,7807} \\
\hline & $S a t_{2}^{n e u}$ & 0,8874 & & & \\
\hline & $\mathrm{Sat}_{3}^{\text {neu }}$ & 0,7858 & & & \\
\hline \multirow{2}{*}{ Wechselabsicht } & $W a b_{1}$ & 0,7608 & \multirow{2}{*}{0,7354} & \multirow{2}{*}{0,8846} & \multirow{2}{*}{0,7933} \\
\hline & $W a b_{2}$ & 0,8619 & & & \\
\hline Wechselvollzug & $W e c$ & 1,0000 & n. v. & 1,0000 & 1,0000 \\
\hline
\end{tabular}

Tabelle 6.5: Beurteilung der Güte der reflektiven Messmodelle: Ladung $\lambda$, Indikatorreliabilität $\lambda^{2}$, Cronbach's $\alpha$, Faktorreliabilität $\rho_{c}$ und durchschnittlich erfasste Varianz AVE

Tab. 6.5 trägt die benötigten Kennzahlen zusammen. Die erste Wertespalte präsentiert die Reliabilität der einzelnen Indikatoren, d.h. die quadrierten Indikatorladungen. Mit Ausnahme von zwei Indikatoren des Involvements liegen alle Indikatorreliabilitätswerte deutlich über 0,4. Die beiden Einträge, die den Wert 0,4 unterschreiten, liegen aber noch über der Schwelle von 0,2. Die zweite Wertespalte der Tab. 6.5 führt die Werte für die interne Konsistenzreliabilität auf, d. h. Cronbach's $\alpha$. Für das Konstrukt Wechselvollzug lässt sich Cronbach's $\alpha$ nicht ermitteln, da nur ein Indikator vorliegt. Alle übrigen $\alpha$ Werte liegen über der in der Marketingforschung üblichen Schwelle von 0,7. Die dritte Wertespalte der Tab. 6.5 enthält die Faktorreliabilitätswerte $\rho_{c}$. Die Faktorreliabilität sämtlicher reflektiver Konstrukte ist größer als 0,8. Dies signalisiert eine hohe Reliabilität. Die vierte Wertespalte der Tab. 6.5 listet die AVE-Werte auf, also die durchschnittlich erfasste Varianz. Sämtliche AVE-Werte liegen über 0,5 und belegen damit eine reliable Konstruktmessung.

Die analysierten Reliabilitätskennzahlen sprechen insgesamt dafür, dass sämtliche reflektiven Konstrukte reliabel gemessen wurden. Es besteht also kein Anlass, wegen geringer Reliabilität auf die zusätzliche Information einiger Indikatoren zu verzichten. 


\begin{tabular}{|c|c|c|c|c|}
\hline \multirow{2}{*}{ Konstrukt } & \multirow{2}{*}{ Indikator } & \multicolumn{3}{|c|}{ Faktor } \\
\hline & & 1 & 2 & 3 \\
\hline \multirow{5}{*}{ Involvement } & $I n v_{1}$ & & 0,7037 & \multirow{5}{*}{0,4380} \\
\hline & $I n v_{2}$ & & 0,7553 & \\
\hline & $\operatorname{Inv}_{3}$ & & 0,6028 & \\
\hline & $\operatorname{Inv}_{4}$ & & 0,7622 & \\
\hline & $\operatorname{Inv}_{5}$ & & 0,6644 & \\
\hline \multirow{6}{*}{ Kundenzufriedenheit } & $S a t_{1}^{a l t}$ & 0,8788 & & \\
\hline & $S a t_{2}^{a l t}$ & 0,8740 & & \\
\hline & $S a t_{3}^{a l t}$ & 0,6473 & & $-0,5078$ \\
\hline & $S a t_{1}^{\text {neu }}$ & 0,8787 & & \\
\hline & $S a t_{2}^{n e u}$ & 0,8689 & & \\
\hline & $\mathrm{Sat}_{3}^{\text {neu }}$ & 0,6538 & & $-0,5306$ \\
\hline \multirow{2}{*}{ Wechselabsicht } & $W a b_{1}$ & & & 0,7713 \\
\hline & $W a b_{2}$ & & & 0,7716 \\
\hline
\end{tabular}

Tabelle 6.6: Komponentenmatrix (Werte kleiner 0,3 sind nicht dargestellt). Rotationsmethode: Varimax mit Kaiser-Normalisierung

\subsubsection{Beurteilung der Validität}

Dieser Unterabschnitt widmet sich der Überprüfung der Validität der Konstruktmessungen. Zunächst werden die reflektiven Konstrukte auf ihre Diskriminanz- und Konvergenzvalidität hin untersucht. Hierzu dient das Fornell-Larcker-Kriterium und eine exploratorische Faktorenanalyse. Dann wird die externe Validität der Messung der Attraktivität der Alternativen analysiert.

Tab. 6.6 zeigt die Ergebnisse der exploratorischen Faktorenanalyse mit anschließender Varimax-Rotation. Aus Gründen der Übersichtlichkeit wurden in dieser Tabelle nur diejenigen Werte aufgetragen, die größer als 0,3 sind. Die Faktorstruktur des Messmodells wird durch diese Zahlen bestätigt: Sämtliche Indikatoren laden am höchsten auf die Konstrukte, denen sie zugeordnet sind, und erst mit deutlichem Abstand auf die übrigen Konstrukte.

Die Varianzerklärung der Hauptkomponente ist in allen Fällen akzeptabel: Die Hauptkomponente der Kundenzufriedenheit (Komponente 1) vereinigt 75,807\% der Varianz der Indikatoren der Kundenzufriedenheit mit dem alten Anbieter und 76,746\% der Varianz 


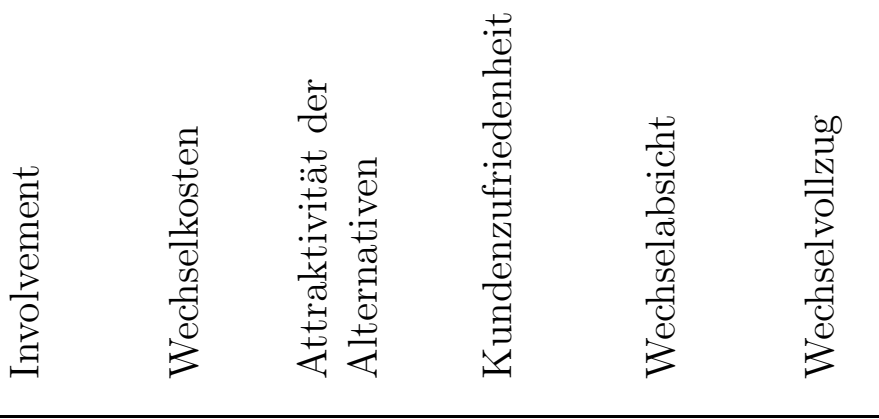

$\begin{array}{lccccccc}\text { Involvement } & 0,5179 & 0,0184 & 0,0157 & 0,0470 & 0,1481 & 0,0743 \\ \text { Wechselkosten } & 0,0184 & n . v . & 0,0438 & 0,0160 & 0,0159 & 0,0742 \\ \text { Attraktivität der Alternativen } & 0,0157 & 0,0438 & n . v . & 0,0057 & 0,0281 & 0,0204 \\ \text { Kundenzufriedenheit } & 0,0470 & 0,0160 & 0,0057 & 0,7626 & 0,1792 & 0,0305 \\ \text { Wechselabsicht } & 0,1481 & 0,0159 & 0,0281 & 0,1792 & 0,7933 & 0,0226 \\ & & & & & & \\ \text { Wechselvollzug } & 0,0743 & 0,0742 & 0,0204 & 0,0305 & 0,0226 & 1,0000\end{array}$

Tabelle 6.7: Überprüfung der Diskriminanzvalidität. Hauptdiagonale: AVE-Werte für reflektive Konstrukte. Übrige Werte: Quadrierte Korrelationen zwischen den Konstrukten. Für die Attraktivität der Alternativen und die Kundenzufriedenheit sind die jeweils kritischeren Werte eingetragen.

der Indikatoren der Kundenzufriedenheit mit dem neuen Anbieter auf sich. Für das Involvement beträgt die Varianzerklärung (Komponente 2) 52,042\%, für die Wechselabsicht (Komponente 3) sind es 79,133\%. Insgesamt kann den reflektiv gemessenen Konstrukten durchgängig Konvergenzvalidität und Unidimensionalität bescheinigt werden.

Tab. 6.7 zeigt eine symmetrische Matrix mit den quadrierten Korrelationen zwischen den einzelnen Konstruktwerten, wobei in der Hauptdiagonalen anstatt Einsen die AVEWerte aufgetragen sind. Aus Tab. 6.7 ist ersichtlich, dass das Modell das Fornell-LarckerKriterium erfüllt: In allen Zeilen bzw. Spalten bildet der AVE-Wert das Zeilen- bzw. Spaltenmaximum. Es bleibt daher festzustellen, dass durchgängig Diskriminanzvalidität vorliegt.

Zur Überprüfung der externen Validität der Messung der Attraktivität der Alternativen wurde mittels PLS-Pfadmodellierung ein Pseudo-MIMIC-Modell geschätzt. Als reflektive 
Indikatoren wurden zwei Indikatoren zur wahrgenommenen Attraktivität der Alternativen verwendet. Die reflektiven Indikatoren erfragten den Grad der Zustimmung der Versuchspersonen zu den folgenden beiden Aussagen:

- $W_{A t t}$ (invers formuliert): „Mein Stromversorger bietet im Vergleich mit anderen Anbietern ein gutes Preis-Leistungs-Verhältnis.“

- $W A t t_{2}$ "Andere Stromversorger wären mir von größerem Nutzen als mein derzeitiger Anbieter."

Abb. 6.11 zeigt das resultierende Pseudo-MIMIC-Modell. Es wurde getestet unter den Versuchspersonen, die ihren Anbieter nicht gewechselt hatten, da sich nur bei den Versuchspersonen, die ihren Anbieter nicht gewechselt hatten, der bewertete Gegenstand („mein Stromversorger" bzw. „mein derzeitiger Anbieter") einem konkreten Anbieter zuordnen ließ. Der Pfadkoeffizient zwischen der objektiv gemessenen Attraktivität der Alternativen und der subjektiv gemessenen wahrgenommenen Attraktivität der Alternativen beträgt 0,5648 und ist auf dem Ein-Promille-Niveau signifikant größer als null. Gemäß RINDSKOPF (1984) kann unter diesen Umständen von externer Validität ausgegangen werden.

\subsection{Beurteilung des Strukturmodells}

Die in Kapitel 4 hergeleiteten Hypothesen (für eine zusammenfassende Darstellung s. Abb. 4.6 auf S. 89) wurden simultan statistisch überprüft, d. h. die Modellparameter für die direkten und die moderierenden Effekte wurden in einem Schritt geschätzt.

Der erste Unterabschnitt (Unterabschnitt 6.5.1) befasst sich mit der Beurteilung der Modellgüte. Der zweite Unterabschnitt (Unterabschnitt 6.5.2) diskutiert die Resultate für die direkten Wirkbeziehungen. Der dritte Unterabschnitt (Unterabschnitt 6.5.3) geht auf die moderierenden Effekte ein.

\subsubsection{Beurteilung der Modellgüte}

Betrachtet man die Bestimmtheitsmaße für die endogenen latenten Variablen, lässt sich von einer akzeptablen Modellanpassung sprechen: Die latente Variable Wechselabsicht weist ein $R^{2}$ von 0,3476 auf, ihr korrigiertes $R^{2}$ beträgt 0,3411. Etwas geringer ist das Bestimmtheitsmaß für die Erklärung des tatsächlichen Wechselvollzugs: Hier beträgt $R^{2}$ 0,1958, und das korrigierte $R^{2}$ nimmt einen Wert von 0,1877 an. Zum Vergleich: Eine 


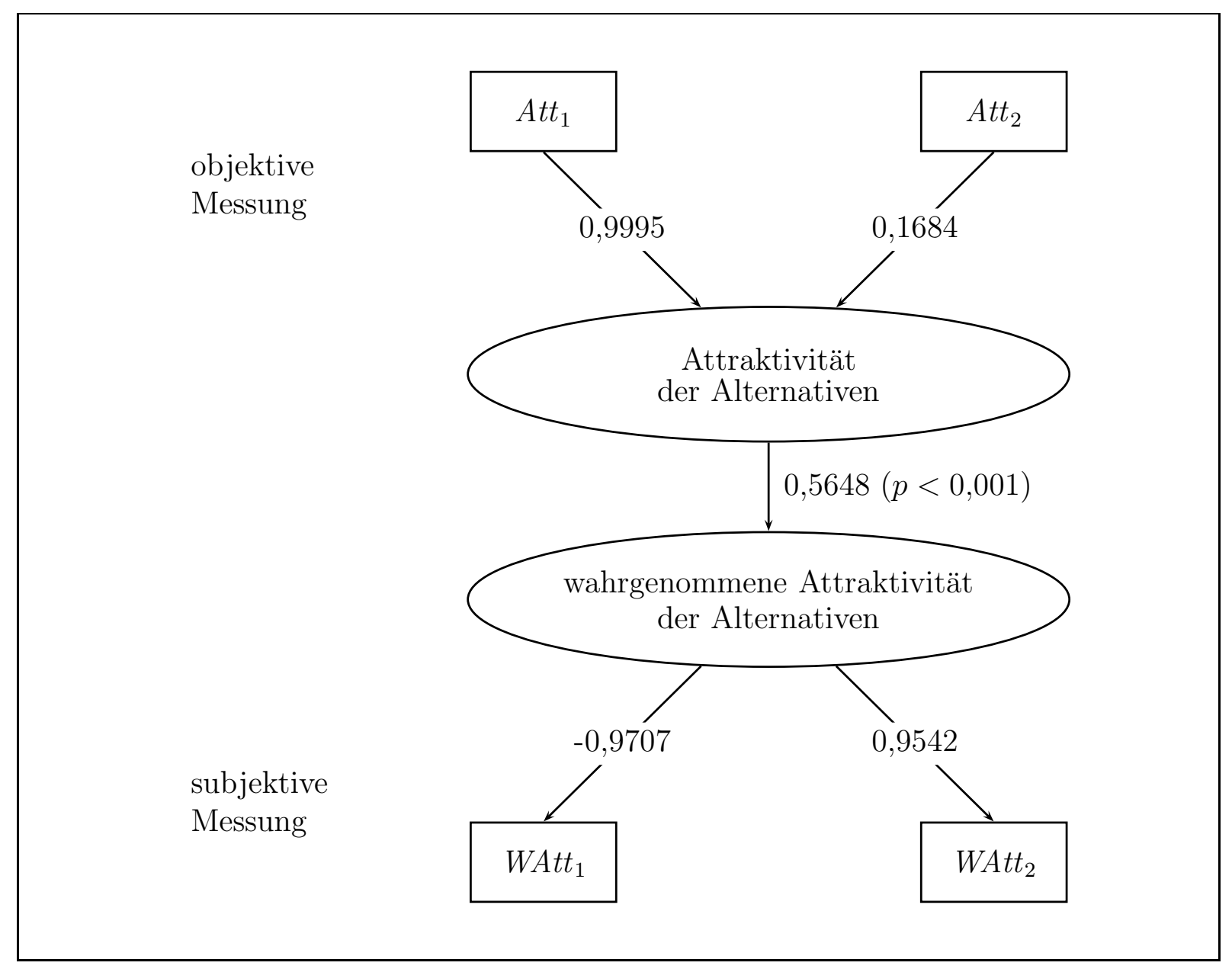

Abbildung 6.11: Überprüfung der externen Validität der formativen Messung der Attraktivität der Alternativen mittels eines Pseudo-MIMIC-Modells

identisch spezifizierte logistische Regression würde hier die folgenden Pseudo- $R^{2}$-Werte liefern: $R_{\text {Cox \& Snell }}^{2}=0,138 ; R_{\text {Nagelkerke }}^{2}=0,307$ und $R_{\text {McFadden }}^{2}=0,248$.

Für die dichotome Variable Wechselvollzug bietet sich eine Beurteilung in Form einer Klassifikationstabelle an, die die Versuchspersonen in Wechsler und Nichtwechsler unterteilt. Die Tabelle 6.8 enthält die entsprechenden Werte für drei Modelle: das Null-Modell, das Modell 1 und das Modell 2.

Das Null-Modell besagt, wie groß der Anteil der korrekt als Wechsler oder Nichtwechsler klassifizierten Versuchspersonen ist, wenn lediglich die Anzahl von Wechslern und Nichtwechslern bekannt ist. Die Entscheidungsregel lautet hier: „Klassifiziere jede Versuchsperson als Nichtwechsler!“ Alle Nichtwechsler werden dann korrekt klassifiziert (100\%), alle Wechsler jedoch inkorrekt (0\%). Gewichtet mit der Zusammensetzung der Stichprobe aus Wechslern und Nichtwechslern resultiert eine Trefferquote (hit ratio) von 91,1\%. Ohne eine Höhergewichtung der Nichtwechsler beträgt die Trefferquote $50 \%$. 


\begin{tabular}{|c|c|c|c|c|c|c|c|c|c|c|}
\hline & & \multicolumn{9}{|c|}{ vorhergesagt } \\
\hline & & \multicolumn{3}{|c|}{ Null-Modell } & \multicolumn{3}{|c|}{ Modell 1} & \multicolumn{3}{|c|}{ Modell 2} \\
\hline \multirow{2}{*}{\multicolumn{2}{|c|}{ beobachtet }} & \multicolumn{2}{|c|}{ Wechsel } & \multirow{2}{*}{$\begin{array}{l}\text { Treffer- } \\
\text { quote }\end{array}$} & \multicolumn{2}{|c|}{ Wechsel } & \multirow{2}{*}{$\begin{array}{l}\text { Treffer- } \\
\text { quote }\end{array}$} & \multicolumn{2}{|c|}{ Wechsel } & \multirow{2}{*}{$\begin{array}{l}\text { Treffer- } \\
\text { quote }\end{array}$} \\
\hline & & nein & ja & & nein & ja & & nein & ja & \\
\hline \multirow{2}{*}{ Wechsel } & nein & 822 & 0 & $100,0 \%$ & 816 & 6 & $99,3 \%$ & 723 & 99 & $88,0 \%$ \\
\hline & ja & 80 & 0 & $0,0 \%$ & 63 & 17 & $21,3 \%$ & 27 & 53 & $66,3 \%$ \\
\hline \multicolumn{2}{|c|}{$\begin{array}{l}\text { Trefferquote } \\
\text { (gewichtet) }\end{array}$} & \multicolumn{3}{|r|}{$91,1 \%$} & \multicolumn{3}{|r|}{$92,4 \%$} & \multicolumn{3}{|r|}{$86,0 \%$} \\
\hline \multicolumn{2}{|c|}{$\begin{array}{l}\text { Trefferquote } \\
\text { (ungewichtet) }\end{array}$} & \multicolumn{3}{|r|}{$50,0 \%$} & \multicolumn{3}{|r|}{$60,3 \%$} & \multicolumn{3}{|r|}{$77,1 \%$} \\
\hline
\end{tabular}

Tabelle 6.8: Klassifikationstabelle für den Wechselvollzug

Auch das Modell 1 hat das Ziel, die maximal mögliche Zahl von Versuchspersonen korrekt zu klassifizieren. Das Modell 1 greift zusätzlich auf die Schätzwerte für den Wechselvollzug zurück. Diese Schätzwerte $z_{i}^{W e c}$ (Index: Versuchsperson $i$ ) sind rationale Zahlen und müssen zunächst dichotomisiert werden. Für die Entscheidungsregel wird eine Schwelle $\zeta_{1}$ verwendet: „Wenn $z_{i}^{W e c} \geq \zeta_{1}$, so klassifiziere die Versuchsperson $i$ als Wechsler, ansonsten als Nichtwechsler!" Folgende Schwelle mit der gewünschten Eigenschaft wurde gefunden: $\zeta_{1}=2,746 \cdot s\left(z_{i}^{W e c}\right)$ mit $s\left(z_{i}^{W e c}\right)$ als der Standardabweichung von $z_{i}^{W e c}$. Ein Gewinn von 21,3 Prozentpunkten bei der Klassifikation der Wechsler geht einher mit einer Abnahme der Trefferquote bei den Nichtwechslern um 0,7 Prozentpunkte. Die gewichtete Trefferquote steigt um 1,2 Prozentpunkte auf 92,3\% Der Klassifikationsfehler reduziert sich somit um über 13 Prozent.

Im Gegensatz zu den vorgenannten Modellen zielt Modell 2 darauf ab, die ungewichtete durchschnittliche Trefferquote zu optimieren, d. h. bei Wechslern und Nichtwechslern soll gleichermaßen eine hohe Trefferquote erreicht werden. Die Häufigkeiten von Wechslern und Nichtwechslern in der Stichprobe werden also bewusst ignoriert. Für die Entscheidungsregel wird eine Schwelle $\zeta_{2}$ verwendet: „Wenn $z_{i}^{W e c} \geq \zeta_{2}$, so klassifiziere die Versuchsperson $i$ als Wechsler, ansonsten als Nichtwechsler!" Für Modell 2 wurde die folgende Schwelle gefunden: $\zeta_{2}=0,722 \cdot s\left(z_{i}^{W e c}\right)$. Um eine Steigerung der Trefferquote bei den Wechslern auf 66,3\% zu erreichen, muss ein Absinken der Trefferquote bei den Nichtwechslern auf $87,8 \%$ in Kauf genommen werden. Die ungewichtete Trefferquote steigt für Modell 2 auf 
$77 \%$. Allerdings sinkt die gewichtete Trefferquote auf nunmehr 85,9\% und stellt somit eine Verschlechterung gegenüber dem Nullmodell dar. Modell 2 ist insbesondere dann von Vorteil, wenn es auf die korrekte Zuordnung der Wechsler ankommt.

\subsubsection{Beurteilung der direkten Effekte}

Dieser Unterabschnitt betrachtet die Ergebnisse in Bezug auf die vermuteten direkten Wirkbeziehungen innerhalb des Wechselverhaltens von Konsumenten im Strommarkt. Die Analyse dieser Hypothesen erfolgt in zwei Schritten: Im ersten Schritt werden die potenziellen Einflussfaktoren der Wechselabsicht betrachtet. Im zweiten Schritt folgen analog die potenziellen Einflussfaktoren des Wechselvollzugs. Tab. 6.9 zeigt die Pfadkoeffizienten und die Gütebeurteilung der direkten Effekte auf die Wechselabsicht, Tab. 6.10 enthält die entsprechenden Werte für die direkten Effekte auf den Wechselvollzug. Die einzelnen Tabelleneinträge sind dabei wie folgt zu verstehen: In der ersten Spalte ist die jeweilige Hypothese genannt, in der zweiten Spalte die zugehörige exogene latente Variable. Die dritte Spalte enthält den zur Hypothese gehörenden Pfadkoeffizienten. Die vierte Spalte gibt an, ob, und wenn ja, auf welchem Signifikanzniveau, die Gegenhypothese verworfen werden kann. Einer Empfehlung von CoHen (1994) folgend zeigt die fünfte Spalte das Konfidenzintervall, innerhalb dessen sich mit 95-prozentiger Wahrscheinlichkeit der wahre Wert für die Stärke der Wirkbeziehung befindet. Die sechste Spalte enthält die Effektgröße $f^{2}$, die als Grundlage zur Beurteilung der Substanzialität dient.

Bestätigt werden kann die Hypothese $\mathrm{H}_{1}$, die einen negativen Wirkzusammenhang zwischen Kundenzufriedenheit und Wechselabsicht vorsieht. Der Pfadkoeffizient von -0,3601 und das Signifikanzniveau von 0,001 sprechen für sich. Die Effektgröße von 0,2337 lässt hier einen mittelgroßen Effekt vermuten.

\begin{tabular}{llcccc}
\hline $\begin{array}{l}\text { Hypo- } \\
\text { these }\end{array}$ & $\begin{array}{l}\text { Antezedenz- } \\
\text { variable }\end{array}$ & $\begin{array}{c}\text { Pfad- } \\
\text { koeffizient }\end{array}$ & $\begin{array}{c}\text { Signi- } \\
\text { fikanz }\end{array}$ & $\begin{array}{c}\text { Konfidenz- } \\
\text { intervall }\end{array}$ & $\begin{array}{c}\text { Effekt- } \\
\text { größe }\end{array}$ \\
\hline $\mathrm{H}_{1}:$ & Kundenzufriedenheit & $-0,3601$ & 0,001 & {$[-0,4244 ;-0,2958]$} & 0,2337 \\
$\mathrm{H}_{3}:$ & $\begin{array}{l}\text { Attraktivität der } \\
\text { Alternativen }\end{array}$ & 0,1174 & 0,001 & {$[0,0669 ; 0,1679]$} & 0,0301 \\
& Wechselkosten & $-0,0639$ & 0,050 & {$[-0,1200 ;-0,0078]$} & 0,0124 \\
$\mathrm{H}_{7}:$ & 0,3318 & 0,001 & {$[0,2803 ; 0,3834]$} & 0,1958 \\
$\mathrm{H}_{11 / 13}:$ & Involvement & & & & \\
\hline
\end{tabular}

Tabelle 6.9: Pfadkoeffizienten der direkten Pfade zur Wechselabsicht 
Die Hypothese $\mathrm{H}_{3}$ erhält ebenfalls Unterstützung durch die Empirie: Die Attraktivität der Alternativen beeinflusst die Wechselabsicht direkt mit einem Pfadkoeffizienten von 0,1174. Auch dieser Effekt ist auf dem Niveau 0,001 signifikant. Allerdings nimmt die Effektgröße $f^{2}$ nur einen Wert von 0,0301 an, d. h. es liegt lediglich ein kleiner Effekt vor.

Als noch geringer erweist sich der in der Hypothese $\mathrm{H}_{7}$ ausgedrückte Wirkzusammenhang zwischen den Wechselkosten und der Wechselabsicht: Zwar werden die Existenz und Richtung der Wirkbeziehung auf dem Fünf-Prozent-Niveau bestätigt. Angesichts des betragsmäßig sehr kleinen Pfadkoeffizienten von -0,0639 und einer geringen Effektgröße von 0,0124 kann jedoch nicht mehr von einem substanziellen Effekt gesprochen werden.

Die konkurrierenden Hypothesen $\mathrm{H}_{11}$ und $\mathrm{H}_{13}$ trafen gegensätzliche Aussagen zur direkten Wirkweise des Involvements auf die Wechselabsicht. Die statistische Analyse deckt einen positiven Zusammenhang zwischen Involvement und Wechselabsicht auf, d. h. sie unterstützt die Hypothese $\mathrm{H}_{13}$, während die Hypothese $\mathrm{H}_{11}$ zu verwerfen ist. Die positive Wirkbeziehung zwischen Involvement und Wechselabsicht manifestiert sich am recht hohen Pfadkoeffizienten von 0,3318. Die vermutete Wirkbeziehung ist auf dem Ein-PromilleNiveau signifikant. Die Effektgröße von 0,1958 spricht für einen mittelgroßen Effekt.

Nachdem die Hypothesen, die die Einflussfaktoren der Wechselabsicht betreffen, überprüft sind, sollen nun die Hypothesen zu den Antezedenzien des Wechselvollzugs getestet werden.

Auch beim Wechselvollzug wird der Einfluss der Kundenzufriedenheit, ausgedrückt in der Hypothese $\mathrm{H}_{2}$, gestützt. Die Kundenzufriedenheit hat einen signifikanten (Signifikanzniveau: 0,010) Einfluss auf den Wechselvollzug. Die Stärke der Wirkbeziehung ist mit $-0,0851$ jedoch deutlich geringer als bei der Wirkung der Kundenzufriedenheit auf die

\begin{tabular}{llcccc}
\hline $\begin{array}{l}\text { Hypo- } \\
\text { these }\end{array}$ & $\begin{array}{l}\text { Antezedenz- } \\
\text { variable }\end{array}$ & $\begin{array}{c}\text { Pfad- } \\
\text { koeffizient }\end{array}$ & $\begin{array}{c}\text { Signi- } \\
\text { fikanz }\end{array}$ & $\begin{array}{c}\text { Konfidenz- } \\
\text { intervall }\end{array}$ & $\begin{array}{c}\text { Effekt- } \\
\text { größe }\end{array}$ \\
\hline $\mathrm{H}_{2}:$ & Kundenzufriedenheit & $-0,0851$ & 0,010 & {$[-0,1436 ;-0,0266]$} & 0,0185 \\
$\mathrm{H}_{4}:$ & $\begin{array}{l}\text { Attraktivität der } \\
\text { Alternativen }\end{array}$ & 0,0644 & 0,050 & {$[0,0041 ; 0,1247]$} & 0,0114 \\
& Wechselkosten & $-0,1881$ & 0,001 & {$[-0,2481 ;-0,1281]$} & 0,0637 \\
$\mathrm{H}_{8}:$ & 0,2143 & 0,001 & {$[0,1570 ; 0,2716]$} & 0,0726 \\
$\mathrm{H}_{12 / 14}:$ & Involvement & & & & \\
\hline
\end{tabular}

Tabelle 6.10: Pfadkoeffizienten der direkten Pfade zum Wechselvollzug 
Wechselabsicht. Ferner ist die Effektgröße mit einem Wert von 0,0185 so gering, dass sich kaum noch von einem substanziellen Effekt sprechen lassen kann.

Noch schwächer ist der Einfluss der Attraktivität der Alternativen: Der Pfadkoeffizient in Höhe von 0,0644 ist zwar auf dem Fünf-Prozent-Niveau signifikant, d. h. eine höhere Attraktivität der Alternativen steht in Zusammenhang mit einem eher vollzogenen Wechsel. Das $f^{2}$ von 0,0114 zeugt jedoch von einer geringen Substanzialität dieses Effekts.

Anders als die beiden vorangegangenen Konstrukte haben die Wechselkosten einen stärkeren Einfluss auf den Wechselvollzug als auf die Wechselabsicht. Der Pfadkoeffizient für die Wirkbeziehung zwischen den Wechselkosten und dem Wechselvollzug (Hypothese $\mathrm{H}_{8}$ ) beträgt -0,1881. Die Hypothese $\mathrm{H}_{8}$ weist darüber hinaus eine Signifikanz von 0,001 auf, ist also hochsignifikant. Angesichts einer Effektgröße von 0,0637 kann allerdings noch nicht von einem mittelgroßen Effekt gesprochen werden.

Analog zur Untersuchung des direkten Einflusses von Involvement auf die Wechselabsicht trafen die konkurrierenden Hypothesen $\mathrm{H}_{12}$ und $\mathrm{H}_{14}$ gegensätzliche Aussagen zur direkten Wirkweise des Involvements auf den Wechselvollzug. Die statistische Analyse deckt auch hier einen positiven Zusammenhang zwischen Involvement und Wechselvollzug auf, d. h. sie unterstützt die Hypothese $\mathrm{H}_{14}$, während die Hypothese $\mathrm{H}_{12}$ zu verwerfen ist. Der positive direkte Zusammenhang zwischen Involvement und Wechselvollzug (Hypothese $\mathrm{H}_{14}$ ) erweist sich als hochsignifikant; dies zeigt sich an der Irrtumswahrscheinlichkeit von 0,001. Der Pfadkoeffizient nimmt einen Wert von 0,2143 an. Er ist somit etwas niedriger als der Pfadkoeffizient der Involvement-Wechselabsicht-Wirkbeziehung (Hypothese $\mathrm{H}_{13}$ ). Die niedrigere Wirkstärke geht mit einer geringeren Substanzialität einher, ausgedrückt durch die Effektstärke in Höhe von 0,0726.

Zusammenfassend lässt sich konstatieren, dass alle vermuteten direkten Wirkbeziehungen empirisch bestätigt werden konnten. Die Kundenzufriedenheit und das Involvement kristallisieren sich hierbei als wesentliche Einflussfaktoren der Wechselabsicht heraus, während zur Erklärung des Wechselvollzugs das Involvement und die Wechselkosten einen entscheidenden Beitrag liefern. Alle direkten Effekte und ihre entsprechenden Pfadkoeffizienten sind in Abb. 6.12 dargestellt.

\subsubsection{Beurteilung der Interaktionseffekte}

Neben den direkten Effekten sollen auch Interaktionseffekte untersucht werden. Ähnlich der Diskussion der direkten Wirkbeziehungen im vorangegangenen Unterabschnitt geht auch dieser Unterabschnitt in zwei Schritten vor: Im ersten Schritt werden die moderierenden Effekte im Zusammenhang mit der Wechselabsicht betrachtet. Im zweiten Schritt 


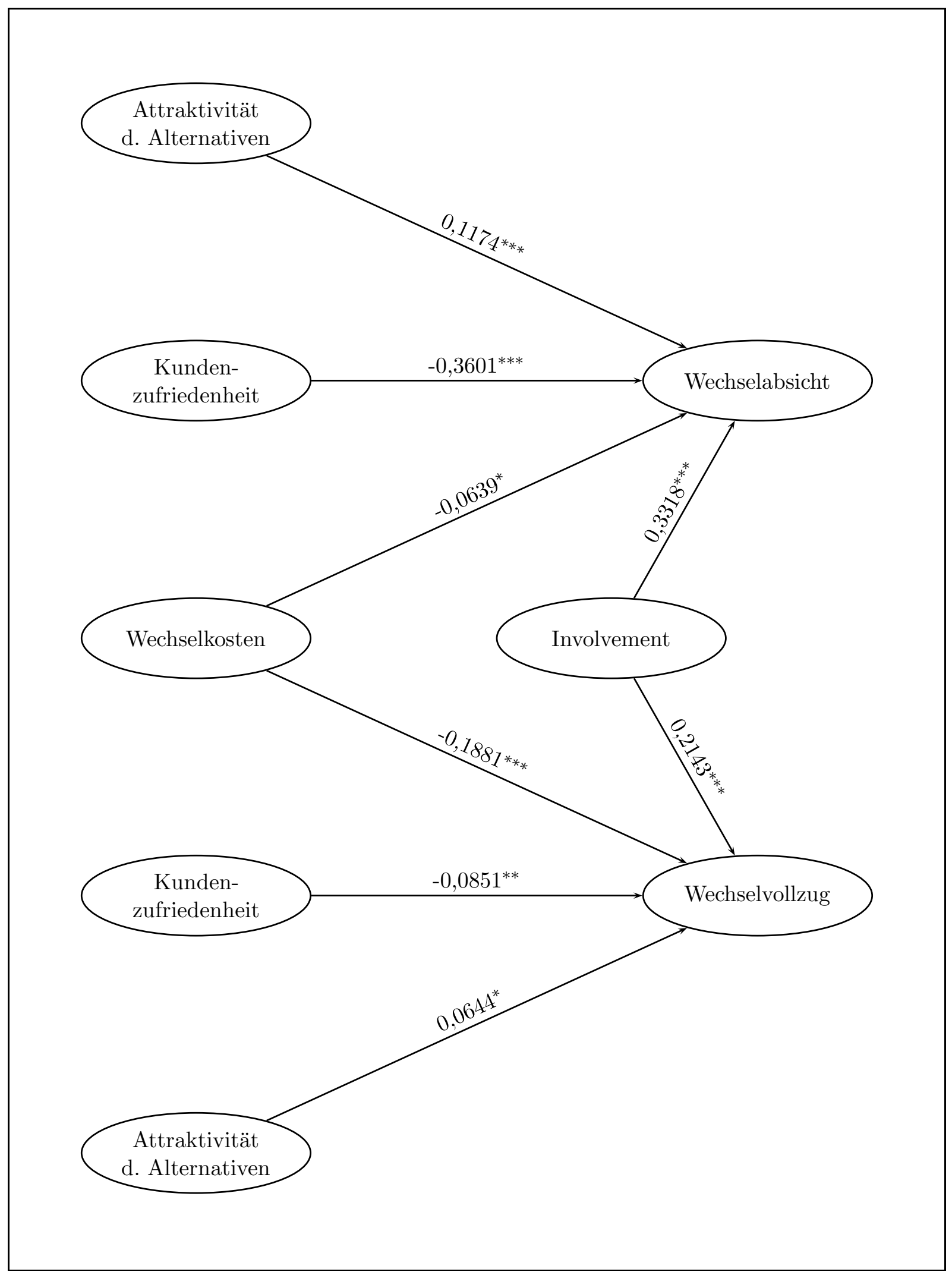

Abbildung 6.12: Stärke der direkten Wirkbeziehungen beim Wechselverhalten von Konsumenten im Markt für Haushaltsstrom

Signifikanzniveaus: ${ }^{* * *} \widehat{=} p<0,001 ;{ }^{* *} \widehat{=} p<0,01 ;{ }^{*} \widehat{=} p<0,05$ 
folgen analog die moderierenden Effekte im Zusammenhang mit dem Wechselvollzug. Tab. 6.11 zeigt die Pfadkoeffizienten der Interaktionsterme sowie deren Gütebeurteilung für die Erklärung der Wechselabsicht. Die einzelnen Tabelleneinträge sind dabei ähnlich wie bei den Tabellen der direkten Wirkbeziehungen zu verstehen: In der ersten Spalte ist die Hypothese dargestellt, in der zweiten Spalte findet sich der jeweilige Interaktionsterm als Produkt von Prädiktor- und Moderatorvariable. Die dritte Spalte enthält den zur Hypothese gehörenden Pfadkoeffizienten. Seine Lesart unterscheidet sich von der einer direkten Beziehung: Der Pfadkoeffizient muss in Verbindung mit dem Pfadkoeffizienten der direkten Beziehung betrachtet werden. Weicht für eine Versuchsperson die Moderatorvariable um eine Standardabweichung nach oben ab, so erhält man die finale Wirkstärke des direkten Effekts als die Summe des Pfadkoeffizienten der direkten Beziehung und des Pfadkoeffizienten der Interaktionsbeziehung; weicht die Moderatorvariable um eine Standardabweichung nach unten ab, so erhält man die finale Wirkstärke des direkten Effekts als die Differenz aus dem Pfadkoeffizienten der direkten Beziehung und dem Pfadkoeffizienten der Interaktionsbeziehung. Die vierte Spalte gibt an, ob, und wenn ja, auf welchem Signifikanzniveau die Gegenhypothese, dass also kein Interaktionseffekt besteht, verworfen werden kann. Die fünfte Spalte zeigt das Konfidenzintervall, innerhalb dessen sich mit 95-prozentiger Wahrscheinlichkeit der wahre Wert für die Stärke der Interaktionsbeziehung befindet. Die sechste Spalte schließlich enthält die Effektgröße $f^{2}$, die als Grundlage zur Beurteilung der Substanzialität dient.

\begin{tabular}{llcccc}
\hline $\begin{array}{l}\text { Hypo- } \\
\text { these }\end{array}$ & Interaktionsterm & $\begin{array}{c}\text { Pfad- } \\
\text { koeffizient }\end{array}$ & $\begin{array}{c}\text { Signi- } \\
\text { fikanz }\end{array}$ & $\begin{array}{c}\text { Konfidenz- } \\
\text { intervall }\end{array}$ & $\begin{array}{c}\text { Effekt- } \\
\text { größe }\end{array}$ \\
\hline $\mathrm{H}_{5}:$ & $\begin{array}{l}\text { Attr. der Alternativen } \times \\
\text { Kundenzufriedenheit }\end{array}$ & $-0,0018$ & n.s. & {$[-0,0749 ; 0,0712]$} & 0,0000 \\
& Wechselkosten $\times$ & 0,0393 & n.s. & {$[-0,0364 ; 0,1149]$} & 0,0022 \\
$\mathrm{H}_{9}:$ & Kundenzufriedenheit & & & & \\
& $\begin{array}{l}\text { Involvement } \times \\
\text { Kundenzufriedenheit }\end{array}$ & $-0,1717$ & 0,001 & {$[-0,2366 ;-0,1068]$} & 0,0483 \\
$\mathrm{H}_{15}:$ & & & & \\
& $\begin{array}{l}\text { Involvement } \times \\
\mathrm{H}_{17}:\end{array}$ & $-0,0726$ & 0,050 & {$[-0,1363 ;-0,0088]$} & 0,0081 \\
& Wechselkosten & & & & \\
$\mathrm{H}_{19}:$ & $\begin{array}{l}\text { Involvement } \times \\
\text { Attr. der Alternativen }\end{array}$ & 0,0455 & n.s. & {$[-0,0209 ; 0,1118]$} & 0,0023 \\
\hline
\end{tabular}

Tabelle 6.11: Interaktionseffekte im Zusammenhang mit der Wechselabsicht 
Die Hypothese $\mathrm{H}_{5}$ unterstellt, dass bei einer höheren Attraktivität der Alternativen eine geringe Kundenzufriedenheit in stärkerem Umfang zur Bildung einer Wechselabsicht führt. Der Pfadkoeffizient von -0,0018 ist nicht nur nicht signifikant kleiner als null, auch liegt der Wert Null nahezu in der Mitte des Konfidenzintervalls. Die Daten liefern also keine Unterstützung dafür, dass der Interaktionseffekt existiert.

Auch die Hypothese $\mathrm{H}_{9}$, die einen Einfluss der Wechselkosten auf die Wirkbeziehung zwischen der Kundenzufriedenheit und der Wechselabsicht vermutet, lässt sich nicht durch die Daten untermauern. Zwar deutet der Pfadkoeffizient von 0,0393 in die vermutete Richtung, d.h. es ist eine leichte Tendenz dafür erkennbar, dass höhere Wechselkosten die Wirkbeziehung zwischen Kundenzufriedenheit und Wechselabsicht schwächen. Jedoch erweist sich der Pfadkoeffizient nicht als signifikant größer als null.

Anders verhält es sich mit der Hypothese $\mathrm{H}_{15}$, die einen Unterschied zwischen der Wirkstärke von latenter und manifester Kundenzufriedenheit auf die Wechselabsicht beschreibt. Diese Hypothese wird durch die Daten deutlich gestützt. Sie ist auf dem EinPromille-Niveau signifikant. Manifeste Kundenzufriedenheit, d.h. Kundenzufriedenheit unter hohem Involvement, vermindert eher die Wechselabsicht als latente Kundenzufriedenheit, d. h. Kundenzufriedenheit unter niedrigem Involvement. Die Hypothese $\mathrm{H}_{15}$ weist mit 0,0483 die höchste Effektgröße unter allen getesteten moderierenden Effekten auf. Betragsmäßig sticht auch der Pfadkoeffizient in Höhe von -0,1717 hervor. Im Vergleich zum Pfadkoeffizienten der moderierten Wirkbeziehung $\left(\mathrm{H}_{1}\right)$ ist er jedoch niedrig. Dadurch bedingt ist es der Moderatorvariablen Involvement nahezu unmöglich, die Wirkbeziehung zwischen der Kundenzufriedenheit und der Wechselabsicht gänzlich aufzuheben.

Bestätigt werden kann ebenfalls die Hypothese $\mathrm{H}_{17}$, die einen Unterschied zwischen der Wirkstärke von latenten und manifesten Wechselkosten auf die Wechselabsicht thematisiert. Sie erweist sich als signifikant auf dem Fünf-Prozent-Niveau. Je höher das Involvement ist, desto eher führen Wechselkosten zu einer verminderten Wechselabsicht. Zwar ist der Pfadkoeffizient mit -0,0726 betragsmäßig klein und die Effektgröße mit 0,0081 gering, jedoch erfüllt die Hypothese $\mathrm{H}_{17}$ eine wichtige Schalterfunktion: Da die direkte Wirkbeziehung zwischen Wechselkosten und Wechselabsicht ebenfalls schwach ist (vgl. die Pfadkoeffizienten für die Hypothese $\mathrm{H}_{7}$ ), entscheidet die Höhe des Involvements darüber, ob Wechselkosten überhaupt zu einer Verminderung der Wechselabsicht führen oder nicht.

Während das Involvement die Wirkstärke von Kundenzufriedenheit und Wechselkosten auf die Wechselabsicht prägt, hat es keinen Einfluss auf die Wirkbeziehung zwischen der Attraktivität der Alternativen und der Wechselabsicht. Dies ergibt die Überprüfung der Hypothese $\mathrm{H}_{19}$, die diesen Interaktionseffekt thematisiert. Zwar hat der Pfadkoeffizient von 0,0455 die richtige Orientierung. Jedoch ist er nicht signifikant größer als null. 
Tab. 6.12 zeigt die Pfadkoeffizienten der Interaktionsterme sowie deren Gütebeurteilung für die Erklärung des Wechselvollzugs. Die Bedeutung der einzelnen Spalten entspricht der von Tab. 6.11.

Die Hypothese $\mathrm{H}_{6}$ vermutet, dass die Attraktivität der Alternativen einen Einfluss auf die Wirkbeziehung zwischen der Kundenzufriedenheit und dem Wechselvollzug hat. Sie kann bestätigt werden (Signifikanzniveau: 0,050). Zwar ist der Pfadkoeffizient von -0,0627 betragsmäßig recht niedrig. Doch angesichts der ebenfalls schwachen moderierten Wirkbeziehung - ihr Pfadkoeffizient beträgt -0,0851 - kommt der Attraktivität der Alternativen eine relativ wichtige Moderatorrolle zu: Während bei einer hohen Attraktivität der Alternative die Kundenzufriedenheit entscheidend für den Wechselvollzug ist, kann eine geringe Attraktivität der Alternativen die Kundenzufriedenheit im Hinblick auf den Wechselvollzug bedeutungslos werden lassen.

Die Hypothese $\mathrm{H}_{10}$ sagt aus, dass die Wechselkosten mit darüber entscheiden, inwieweit die Kundenzufriedenheit den Wechselvollzug beeinflusst. Der Schätzer für die Hypothese $\mathrm{H}_{10}$ erhält einen Wert von 0,1018 und meistert damit das Ein-Prozent-Signifikanzniveau. In Anbetracht der Orientierung des Pfadkoeffizienten lässt sich festhalten, dass hohe wahrgenommene Wechselkosten die Wirkbeziehung zwischen Kundenzufriedenheit und Wechselvollzug schwächen. Dieser Interaktionseffekt ist aufgrund des $f^{2}$-Wertes von 0,0237 als durchaus substanziell anzusehen.

\begin{tabular}{llcccc}
\hline $\begin{array}{l}\text { Hypo- } \\
\text { these }\end{array}$ & Interaktionsterm & $\begin{array}{c}\text { Pfad- } \\
\text { koeffizient }\end{array}$ & $\begin{array}{c}\text { Signi- } \\
\text { fikanz }\end{array}$ & $\begin{array}{c}\text { Konfidenz- } \\
\text { intervall }\end{array}$ & $\begin{array}{c}\text { Effekt- } \\
\text { größe }\end{array}$ \\
\hline $\mathrm{H}_{6}:$ & $\begin{array}{l}\text { Attr. der Alternativen } \times \\
\text { Kundenzufriedenheit }\end{array}$ & $-0,0627$ & 0,050 & {$[-0,1294 ; 0,0039]$} & 0,0041 \\
& Wechselkosten $\times$ & 0,1018 & 0,010 & {$[0,0276 ; 0,1760]$} & 0,0237 \\
$\mathrm{H}_{10}:$ & $\begin{array}{l}\text { Kundenzufriedenheit } \\
\end{array}$ & & & & \\
$\mathrm{H}_{16}:$ & $\begin{array}{l}\text { Involvement } \times \\
\text { Kundenzufriedenheit }\end{array}$ & $-0,0582$ & 0,050 & {$[-0,1217 ; 0,0053]$} & 0,0099 \\
$\mathrm{H}_{18}:$ & $\begin{array}{l}\text { Involvement } \times \\
\text { Wechselkosten }\end{array}$ & $-0,1236$ & 0,001 & {$[-0,1964 ;-0,0508]$} & 0,0329 \\
$\mathrm{H}_{20}:$ & $\begin{array}{l}\text { Involvement } \times \\
\text { Attr. der Alternativen }\end{array}$ & & & & \\
\hline
\end{tabular}

Tabelle 6.12: Interaktionseffekte im Zusammenhang mit dem Wechselvollzug 
Der Unterschied in der Wirkung von latenter und manifester Kundenzufriedenheit auf den Wechselvollzug, der in Hypothese $\mathrm{H}_{16}$ ausgedrückt ist, erweist sich als signifikant mit einer Irrtumswahrscheinlichkeit von 0,050. Die Wirkrichtung entspricht den Erwartungen. Allerdings deuten der Pfadkoeffizient von -0,0582 und insbesondere die Effektgröße von 0,0099 auf einen allenfalls sehr kleinen Interaktionseffekt hin. Der Unterschied zwischen manifester Kundenzufriedenheit und latenter Kundenzufriedenheit hinsichtlich der Wirkung auf den Wechselvollzug existiert demnach, ist aber angesichts seiner geringen Stärke als wenig bedeutsam einzuschätzen.

Die Hypothese $\mathrm{H}_{18}$ über den Unterschied in der Wirkung von manifesten und latenten Wechselkosten auf den Wechselvollzug erweist sich auf dem Ein-Promille-Niveau als signifikant. Der entsprechende Pfadkoeffizient beträgt -0,1236. Ein hohes Involvement verstärkt also die Wirkbeziehung zwischen Wechselkosten und Wechselvollzug. Mit anderen Worten: Manifeste Wechselkosten verhindern eher den Wechselvollzug als latente Wechselkosten. Die Effektgröße von 0,0328 spricht für einen kleinen Effekt.

Die Hypothese $\mathrm{H}_{20}$ wird durch die Daten nicht untermauert. Der Pfadkoeffizient von 0,0525 ist nicht nur recht klein, er lässt sich auch nicht signifikant von Null unterscheiden. Es lässt sich somit nicht bestätigen, dass das Involvement auf die Beziehung zwischen der Attraktivität der Alternativen und dem Wechselvollzug einwirkt.

Abb. 6.13 zeigt die geschätzten Parameter für die direkten und die moderierenden Wirkbeziehungen in einem gemeinsamen Kontext. Zur Erhöhung der Übersichtlichkeit enthält die Abbildung nur diejenigen Wirkbeziehungen, die sich als signifikant erwiesen haben.

\subsection{Differenzen der Parameter von Wechselabsicht und Wechselvollzug}

Die Zielsetzung dieser Arbeit umfasst nicht nur eine kausal orientierte Untersuchung der Antezedenzvariablen von Wechselabsicht und Wechselvollzug, wie sie in den vorangegangenen Abschnitten durchgeführt wurde. Auch soll eine explorative Analyse aufzeigen, wo Diskrepanzen zwischen den relevanten Einflussgrößen von Wechselabsicht und Wechselvollzug vorliegen. Mitunter lassen sich daraus Schlüsse ziehen, warum Konsumenten ihren Anbieter nicht wechseln, selbst wenn sie die Absicht dazu haben.

Dieser Abschnitt untersucht die Unterschiede zwischen den Wirkstärken der Antezedenzvariablen in zwei Schritten: Zunächst werden die direkten Effekte betrachtet, dann wird auf die moderierenden Effekte eingegangen. 


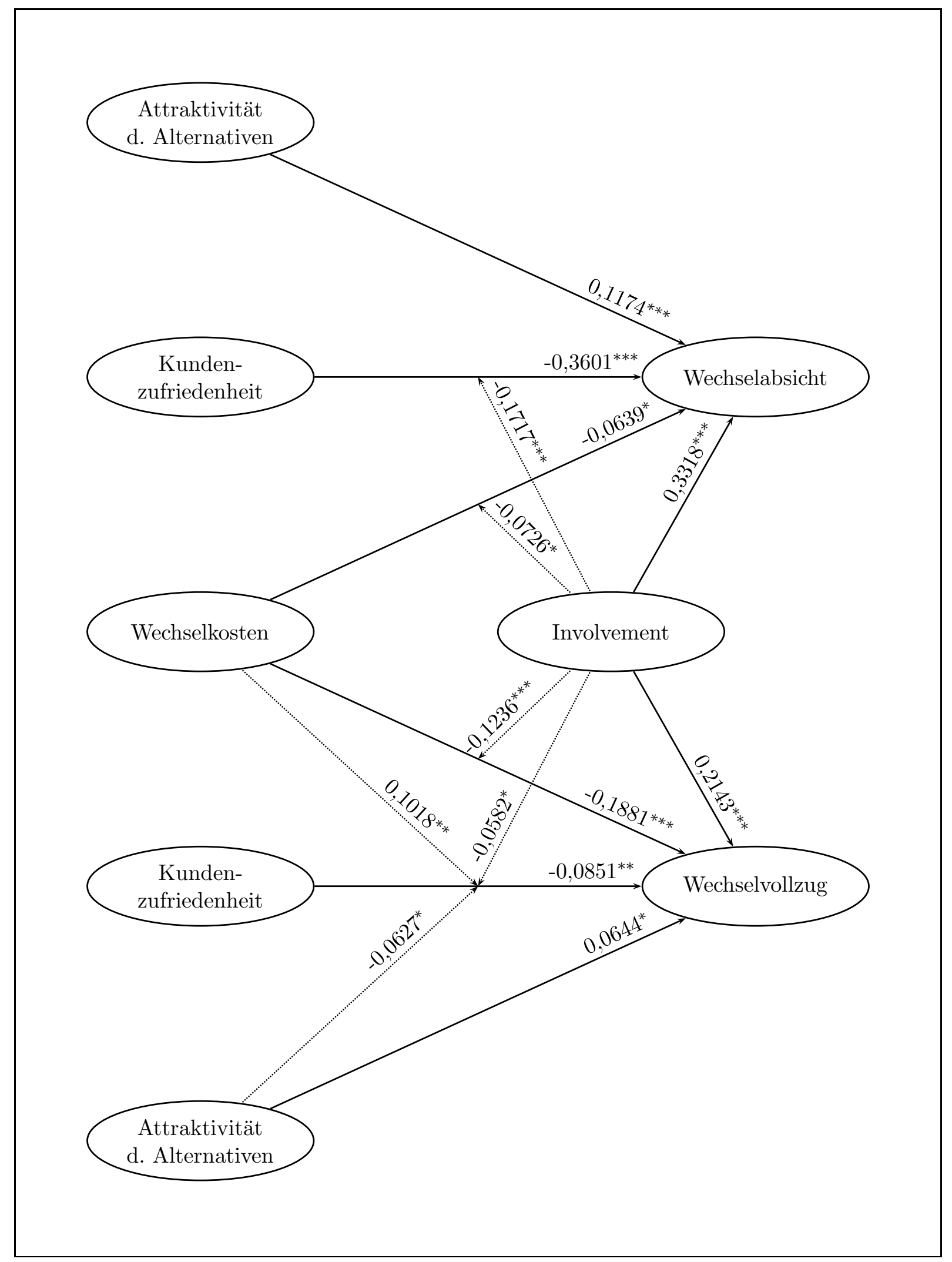

Abbildung 6.13: Signifikante direkte und moderierende Effekte beim Wechselverhalten von Konsumenten im Markt für Haushaltsstrom

Signifikanzniveaus: ${ }^{* * *} \widehat{=} p<0,001 ;{ }^{* *} \widehat{=} p<0,01 ;{ }^{*} \widehat{=} p<0,05$ 
Für die direkten Effekte wurde die maßgebliche Information in Tab. 6.13 zusammengestellt. Für jede exogene latente Variable wird in der ersten Wertespalte der Pfadkoeffizient $\beta^{\text {Wechselabsicht }}$ für den Pfad zur Wechselabsicht mit dem erreichten Signifikanzniveau angegeben. Diese Werte sind der Tab. 6.9 entnommen. Die zweite Wertespalte von Tab. 6.13

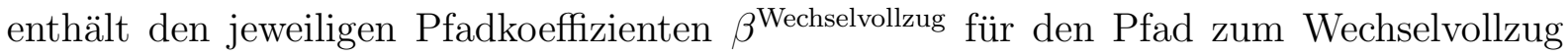
mit dem erreichten Signifikanzniveau. Diese Werte sind aus Tab. 6.10 bekannt. Die dritte Wertespalte bildet den Differenzbetrag $\Delta$ aus den beiden linken Werten. Hieraus lässt sich erkennen, in welchem Maße sich der Einfluss der exogenen latenten Variablen auf die Wechselabsicht und den Wechselvollzug unterscheiden. Darüber hinaus ist es wichtig zu wissen, ob die gefundenen Unterschiede signifikant von null verschieden sind. Diesen Zweck erfüllt der paarweise Vergleich der aus dem Bootstrapping gewonnenen Regressionsparameter $\beta_{i}^{\text {Wechselabsicht }}$ und $\beta_{i}^{\text {Wechselvollzug }}$. KRENGEL (1991, S. 175) empfiehlt, bei einer solchen verbundenen Stichprobe jeweils die Differenzen zu bilden:

$$
\Delta_{i}=\beta_{i}^{\text {Wechselabsicht }}-\beta_{i}^{\text {Wechselvollzug }}
$$

Damit reduziert sich das Problem auf ein Einstichprobenproblem. Mit Hilfe der Standardabweichung der Differenzen $s\left(\Delta_{i}\right)$ lässt sich schließlich das Signifikanzniveau ermitteln.

Die Analyse der Parameterdifferenzen kommt für die direkten Effekte zu folgendem Ergebnis: Auch wenn alle direkten Effekte an sich signifikant sind, so ergeben sich doch drei signifikante Unterschiede in der Wirkstärke der exogenen latenten Variablen auf die Wechselabsicht und den Wechselvollzug:

- Der größte Unterschied findet sich bei der Kundenzufriedenheit: Sie trägt wesentlich mehr zur Erklärung der Wechselabsicht als zur Erklärung des Wechselvollzugs bei.

\begin{tabular}{llll}
\hline Erklärende Variable & $\beta^{\text {Wechselabsicht }}$ & $\beta^{\text {Wechselvollzug }}$ & $\boldsymbol{\Delta}$ \\
\hline Kundenzufriedenheit & $-0,3601^{* * *}$ & $-0,0851^{* *}$ & $0,2750^{* * *}$ \\
Attraktivität der Alternativen & $0,1174^{* * *}$ & $0,0644^{*}$ & 0,0530 \\
wahrgenommene Wechselkosten & $-0,0639^{*}$ & $-0,1881^{* * *}$ & $0,1243^{* *}$ \\
Involvement & $0,3318^{* * *}$ & $0,2143^{* * *}$ & $0,1175^{* *}$ \\
\hline
\end{tabular}

Tabelle 6.13: Vergleich der Regressionskoeffizienten der direkten Effekte bei Wechselabsicht und Wechselvollzug

Signifikanzniveaus: ${ }^{* * *} \widehat{=} p<0,001 ;{ }^{* *} \widehat{=} p<0,01 ;{ }^{*} \widehat{=} p<0,05$ 
- Ein kleinerer, aber doch wichtiger Unterschied zeigt sich bei den Wechselkosten: Sie erklären als einzige exogene latente Variable den Wechselvollzug besser als die Wechselabsicht.

- Ein weiterer kleiner Unterschied ist bei der Wirkstärke des Involvements festzustellen: Es erklärt die Wechselabsicht signifikant besser als den Wechselvollzug.

Eine Besonderheit stellt die Attraktivität der Alternativen dar. Zwar liefern bei diesem Konstrukt die Pfadkoeffizienten keinerlei Hinweis auf einen strukturellen Unterschied zwischen der Erklärung der Wechselabsicht und des Wechselvollzugs. Jedoch zeigt hier das Messmodell deutliche Unterschiede (vgl. Tab. 6.4): Wenn es um die Erklärung der Wechselabsicht geht, bestimmt der Stromverbrauch - operationalisiert durch die Haushaltsgröße $\left(A t t_{2}\right)$ - die Attraktivität der Alternativen. Hingegen erweist sich die erzielbare Ersparnis - operationalisiert durch den Anbieter $\left(A t t_{1}\right)$ - als entscheidende Determinante der Attraktivität der Alternativen, wenn es sich um die Erklärung des Wechselvollzugs dreht.

Für die Unterschiede in den Wirkstärken der moderierenden Effekte mit der Wechselabsicht und dem Wechselvollzug als endogenen Variablen liefert Tab. 6.14 die relevante Information. Die ersten beiden Wertespalten fassen die Pfadkoeffizienten und deren Signifikanzniveaus aus den Tabellen 6.11 und 6.12 zusammen. Die dritte Wertespalte zeigt die Differenzbeträge zwischen den Pfadkoeffizienten sowie deren Signifikanzniveaus.

\begin{tabular}{lllc}
\hline Erklärende Interaktion & $\beta^{\text {Wechselabsicht }}$ & $\beta^{\text {Wechselvollzug }}$ & $\Delta$ \\
\hline $\begin{array}{l}\text { Kundenzufriedenheit } \times \text { Attraktivität der } \\
\text { Alternativen }\end{array}$ & $-0,0018$ & $-0,0627^{*}$ & 0,0609 \\
$\begin{array}{l}\text { Kundenzufriedenheit } \times \text { wahrgenommene } \\
\text { Wechselkosten }\end{array}$ & 0,0393 & $0,1018^{* *}$ & 0,0625 \\
$\begin{array}{l}\text { Involvement } \times \text { Kundenzufriedenheit } \\
\quad \text { Attraktivität }\end{array}$ & $-0,1717^{* * *}$ & $-0,0582^{*}$ & $0,1135^{* * *}$ \\
$\begin{array}{l}\text { Involvement } \times \\
\text { der Alternativen }\end{array}$ & 0,0525 & 0,0070 \\
$\begin{array}{l}\text { Involvement } \times \\
\text { wahrgenommene Wechselkosten }\end{array}$ & $-0,0726^{*}$ & $-0,1236^{* * *}$ & 0,0510 \\
\hline
\end{tabular}

Tabelle 6.14: Vergleich der Regressionskoeffizienten der moderierenden Effekte bei Wechselabsicht und Wechselvollzug Signifikanzniveaus: ${ }^{* *} \widehat{=} p<0,001 ;{ }^{* *} \widehat{=} p<0,01 ;{ }^{*} \widehat{=} p<0,05$ 
Obwohl bei der Erklärung des Wechselvollzugs einige Interaktionsterme signifikant sind, die bei der Erklärung der Wechselabsicht nicht signifikant sind, erweist sich nur eine einzige Parameterdifferenz als signifikant: Die Interaktion von Kundenzufriedenheit und Involvement erklärt signifikant stärker die Wechselabsicht als den Wechselvollzug. Demnach wirken manifeste und latente Kundenzufriedenheit unterschiedlicher auf die Wechselabsicht ein als auf den Wechselvollzug. 


\section{Schlussbetrachtung}

Die Ergebnisse dieser Arbeit leisten einen Beitrag sowohl für die Marketingwissenschaft (Abschnitt 7.1) als auch für die Marketingpraxis (Abschnitt 7.2). Die Grenzen der vorliegenden Arbeit und sich daraus ergebende Anknüpfungspunkte für die weitere Forschung werden im letzten Abschnitt (Abschnitt 7.3) aufgezeigt.

\subsection{Implikationen für die Marketingwissenschaft}

Die in dieser Arbeit gewonnenen Erkenntnisse haben zahlreiche Implikationen für die Marketingwissenschaft. Diese werden nachfolgend herausgearbeitet. Dabei wird Bezug genommen auf die einzelnen als Determinanten des Wechselverhaltens identifizierten Konstrukte.

Die Wirkbeziehung zwischen Kundenzufriedenheit und Wechselabsicht erfährt in der vorliegenden Arbeit einmal mehr eine Bestätigung. Damit reiht sich diese empirische Untersuchung in die Vielzahl von ähnlichen Studien ein, die den Zusammenhang zwischen Kundenzufriedenheit und Wechselabsicht untermauern. Interessant ist das empirische Resultat, dass sich die Kundenzufriedenheit mit dem aktuellen Anbieter auf den Wechselvollzug deutlich schwächer auswirkt als auf die Wechselabsicht. Zum einen lässt der geringe Zusammenhang zwischen der Kundenzufriedenheit und dem Wechselvollzug zu, dass selbst Konsumenten, die mit ihrem Anbieter unzufrieden sind, in vielen Fällen ihren Anbieter beibehalten. Zum anderen demonstriert dies, wie wichtig es ist, im Rahmen der Modellierung des Wechselverhaltens nicht allein auf die Verhaltensabsicht zu achten, sondern auch das tatsächliche Verhalten zu analysieren. Die Kundenzufriedenheit erweist sich nicht als die allein maßgebliche Determinante des Wechselverhaltens.

Als eine zweite Determinante des Wechselverhaltens ist die Attraktivität der Alternativen zu nennen. Für Konsumenten spielt die Höhe des durch einen Anbieterwechsel erzielbaren Wechselgewinns also eine signifikante Rolle, und das sowohl bei der Bildung der Wechselabsicht als auch beim Wechselvollzug. Dies hat zugleich auch Auswirkungen auf die Gestaltung zukünftiger Arbeiten zur Kundenloyalität: Während die Attraktivität der Alternativen bei der einstellungsorientierten Konzeptualisierung von Kundenloyalität explizit außer Acht gelassen wird (vgl. S. 21), sollte sie bei verhaltensorientierten Konzeptualisierungen stets berücksichtigt werden. Des Weiteren erweist sich die Attraktivität der 
Alternativen als ein signifikanter Moderator der Wirkbeziehung zwischen Kundenzufriedenheit und Wechselvollzug. Eine derart gestaltete Interaktion zwischen der Kundenzufriedenheit und der Attraktivität der Alternativen hat bislang in der Marketingforschung noch zu geringe Beachtung gefunden.

Betrachtet man allein die Wirkbeziehung zwischen den Wechselkosten und der Wechselabsicht, scheinen Wechselkosten nur eine kleine Rolle im Rahmen des Wechselverhaltens von Konsumenten im Strommarkt zu spielen. Erst wenn man den Wechselvollzug und dessen Determinanten analysiert, wird die Bedeutung der Wechselkosten offenbar. Übereinstimmend mit den Ergebnissen von Jones ET AL. (2000) stellt sich der direkte Aufwand eines Wechsels als die wichtigste Komponente der Wechselkosten heraus; mit etwas Abstand folgen die Such- und Informationskosten sowie das wahrgenommene Risiko.

Möglicherweise spielen Wechselkosten in vielen Branchen eine wesentliche Rolle beim Wechselverhalten von Konsumenten. Studien zum Konsumentenverhalten und zur Kundenloyalität, die sich allein mit der Messung von Verhaltensabsichten und deren Determinanten beschäftigen, also eine Untersuchung des tatsächlichen Verhaltens auslassen, vermögen den wirklichen Einfluss der Wechselkosten nicht aufzuzeigen. Aufgrund der Dominanz von Untersuchungen der letztgenannten Art ist es denkbar, dass in der Marketingwissenschaft eine systematische Verzerrung dergestalt existiert, dass der Einfluss der Wechselkosten im Rahmen des Wechselverhaltens von Konsumenten - und vielleicht auch darüber hinaus - unterschätzt wird. Zumindest im Rahmen des Wechselverhaltens von Konsumenten im Strommarkt stehen die Wechselkosten der Kundenzufriedenheit als Erklärungsansatz in nichts nach. Es bleibt zu wünschen, dass die Wechselkosten in Zukunft eine ähnlich hohe Aufmerksamkeit erhalten wie die Kundenzufriedenheit.

Ein höheres Involvement geht einher mit einer stärkeren Absicht, den Anbieter zu wechseln, und einer größeren Wahrscheinlichkeit, dass der Anbieter gewechselt wird. Dieses Ergebnis der empirischen Untersuchung überrascht umso mehr, als es offenbar in Widerspruch zu den Erkenntnissen von Bloemer und KASPER (1995, 1999) steht, nämlich dass Involvement zu einer höheren Kundenloyalität führt. Will man diesen Widerspruch aufösen, bieten sich drei Erklärungen an: Erstens sind die Konstrukte Wechselabsicht und Kundenloyalität möglicherweise inhaltlich derart weit voneinander entfernt, dass sie entgegengesetzte Antezedenzvariablen aufweisen. Zweitens kann es sein, dass die Wirkweise des Involvements nicht linear ist, sondern einer U-Kurve folgt, so dass die vorliegende Arbeit und die Untersuchung von BLOEMER und KASPER jeweils lediglich einen Teil des Involvement-Spektrums analysieren. Drittens beschränken sich die Erkenntnisse von BlOemer und KASPER eventuell auf Kunden, die ihren Anbieter aus einer größeren Menge potenzieller Anbieter ausgesucht haben. Mitunter lässt sich damit das loyale Ver- 
halten von Kunden nicht erklären, wenn diese allein aus historischen Gründen bei einem bestimmten Anbieter sind, ohne jemals eine Auswahlentscheidung getroffen zu haben.

Die empirische Untersuchung der moderierenden Effekte konnte erneut bestätigen, dass sich die Wirkungen von manifester und latenter Kundenzufriedenheit unterscheiden. Darüber hinaus wurde deutlich, dass nicht nur bei der Kundenzufriedenheit zwischen einer manifesten und einer latenten Ausprägung differenziert werden kann, sondern auch bei den Wechselkosten: Analog zur Kundenzufriedenheit zeigten sich Unterschiede in der Wirkweise zwischen manifesten und latenten Wechselkosten. Sowohl die direkten als auch die indirekten Effekte des Involvements legen Zeugnis vom Erklärungspotenzial des Involvements ab. Sie stützen damit die Forderung nach einer intensiveren Miteinbeziehung des Involvementkonstrukts in die Marketingforschung im Allgemeinen und in die Erforschung von Wechselverhalten im Besonderen.

Die Austauschtheorie hat sich als gute Grundlage zur Modellierung des Wechselverhaltens bei Versorgungsbeziehungen erwiesen. Ein wichtiger Aspekt ist hierbei, dass die aus ihr abgeleiteten Konstrukte Kundenzufriedenheit, wahrgenommene Wechselkosten und Attraktivität der Alternativen einen signifikanten Teil des Wechselverhaltens erklären. Weiterhin steht sie in Einklang mit der unterschiedlichen Wirkstärke von Kundenzufriedenheit und wahrgenommenen Wechselkosten bei Wechselabsicht und Wechselvollzug. Auf

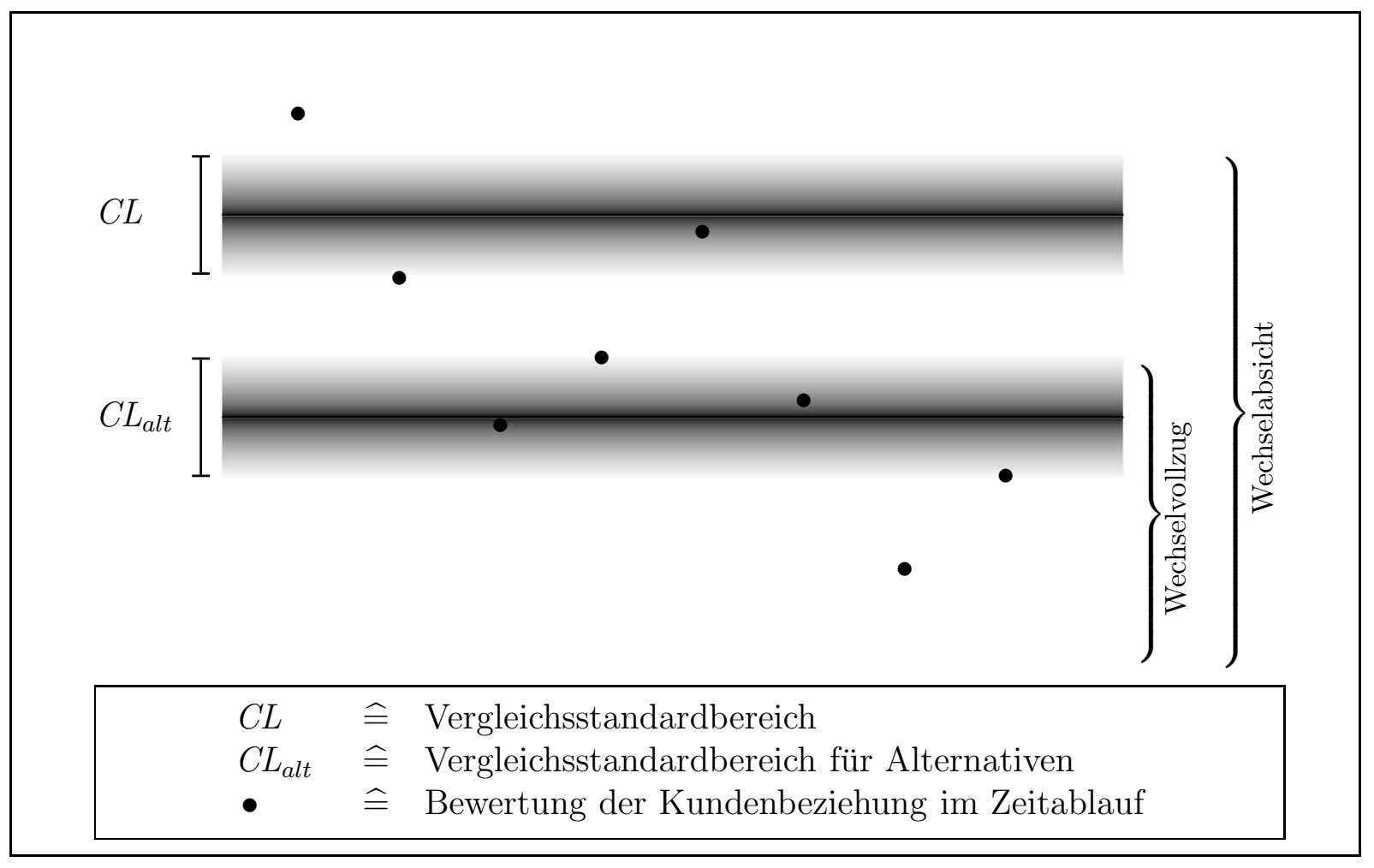

Abbildung 7.1: Ein modifiziertes Modell zur Austauschtheorie 
der rechten Seite in Abb. 7.1 ist aufgetragen, durch welche Umstände die Wechselabsicht und der Wechselvollzug begünstigt werden. Eine dunklere Schattierung spricht für eine größere Wechselabsicht bzw. einen wahrscheinlicheren Wechselvollzug. Es kann demnach eine Wechselabsicht eintreten, sobald die Versorgungsbeziehung nicht mehr den Erwartungen entspricht, d. h. wenn keine Kundenzufriedenheit mehr vorliegt. Der Vollzug des Anbieterwechsels hängt jedoch maßgeblich vom Vergleichsstandard für Alternativen ab. Bei der Abwägung über den tatsächlichen Anbieterwechsel spielen die wahrgenommenen Wechselkosten eine entscheidende Rolle. Es ist daher plausibel, dass die Höhe der Kundenzufriedenheit besonders die Wechselabsicht erklärt, während die wahrgenommenen Wechselkosten über den Wechselvollzug entscheiden.

Bisher findet das Involvement innerhalb der Austauschtheorie keine Beachtung. Ausgehend von der empirisch nachgewiesenen Moderatorwirkung des Involvements für die Wirkung von Kundenzufriedenheit und wahrgenommenen Wechselkosten lässt sich die Austauschtheorie folgendermaßen erweitern: Bei den beiden Vergleichsstandards $C L$ und $C L_{\text {alt }}$ handelt es sich nicht um exakte Grenzlinien, sondern vielmehr um Bereiche. Innerhalb dieser Bereiche ist das Konsumentenverhalten nur schwer vorhersagbar. Die Ausdehnung der Bereiche und damit auch die Trennschärfe der Vergleichsstandards hängen von der Höhe des Involvements ab: Je höher das Involvement, desto kleiner sind die Vergleichsstandardbereiche, und desto schärfer trennen sie. Abb. 7.1 stellt die vorgeschlagene Erweiterung graphisch dar.

\subsection{Implikationen für die Marketingpraxis}

Die im Rahmen der vorliegenden Arbeit gewonnenen Erkenntnisse zum Wechselverhalten von Konsumenten im Strommarkt haben vielfältige Implikationen für die Marketingpraxis. Abb. 7.2 zeigt, welche Strategieoptionen Unternehmen im Markt offenstehen. Zum

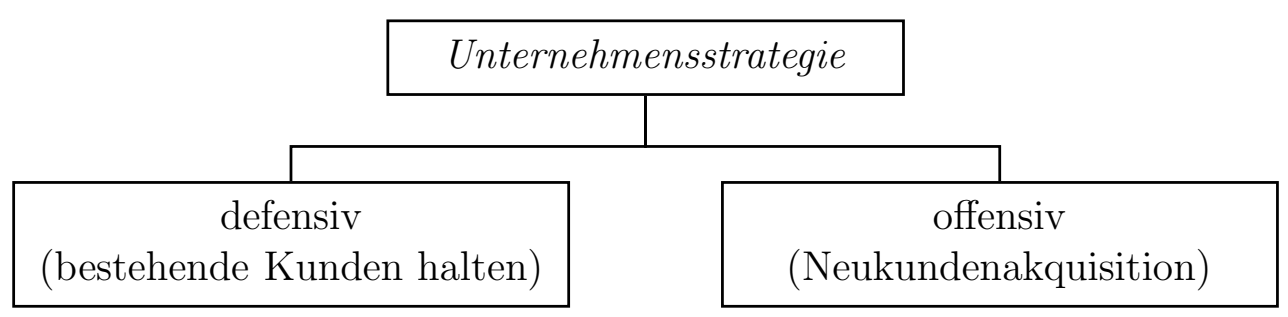

Abbildung 7.2: Strategieoptionen eines Anbieters (vgl. Fornell, 1992, S. 8) 
einen kann ein Unternehmen aggressiv am Markt auftreten, um Kundenakquisition zu betreiben. Zum anderen kann das Unternehmen eine defensive Strategie wählen, die helfen soll, die aktuelle Kundenbasis zu halten (Fornell, 1992, S. 7). Im Strommarkt für Haushaltsstromkunden sind die Strategien vorwiegend historisch bedingt festgelegt, und es lassen sich zwei Positionen identifizieren, die Stromanbieter einnehmen können: Dies ist erstens die Position der "Verteidiger". In ihr befinden sich Anbieter, die bereits über einen Kundenstamm verfügen und ihre Kunden halten wollen. Speziell im Strommarkt für Haushaltsstromkunden handelt es sich hierbei um die Stromanbieter, die ehemals das Versorgungsmonopol für ein bestimmtes Gebiet besaßen und nun ihren Marktanteil verteidigen wollen. Die zweite Position ist die der "Angreifer". In ihr befinden sich Anbieter, die Kunden von anderen Anbietern abwerben wollen. Im Strommarkt setzen sich die Angreifer zum einen aus neuen Anbietern und zum anderen aus denjenigen Ex-Monopolisten zusammen, die auch außerhalb ihrer ehemaligen Gebietsgrenzen Neukunden akquirieren. Sowohl für Unternehmen in der Verteidigerposition als auch für Unternehmen in der Angreiferposition sind die in dieser Arbeit gewonnenen Erkenntnisse relevant.

Die folgenden Unterabschnitte beleuchten die Erkenntnisse dieser Arbeit aus der Perspektive der jeweiligen Position. Unter der Prämisse positionsspezifischer Zielsetzungen lassen sich Handlungsempfehlungen für Verteidiger und Angreifer ableiten. Unterabschnitt 7.2.1 beschäftigt sich mit den Implikationen für Unternehmen in der Verteidigerposition; Unterabschnitt 7.2.2 mit den Implikationen für Unternehmen in der Angreiferposition.

\subsubsection{Implikationen für Unternehmen in der Verteidigerposition}

Seitdem Privatkunden ihren Stromanbieter frei wählen können, rücken aus Sicht der etablierten Anbieter Maßnahmen zur Sicherung der bestehenden Kundenbeziehungen sowie die Bedürfnisse und Erwartungen der Kunden mehr und mehr in den Vordergrund (Töpfer, 2001, S. 191; Cornelsen und Litzenroth, 2002, S. 40). Die Ergebnisse dieser Arbeit untermauern die Bedeutung der Kundenzufriedenheit: Zufriedenere Kunden entwickeln in geringerem Ausmaß Wechselabsichten als unzufriedene Kunden. Ein ähnlicher, allerdings schwächerer Zusammenhang besteht auch zwischen Kundenzufriedenheit und Wechselvollzug. Unter gewissen Umständen ist aber auch dieser Zusammenhang stark:

- wenn Konsumenten hoch involviert sind,

- wenn sie die mit einem Anbieterwechsel verbundenen Wechselkosten als niedrig erachten oder

- wenn sie die alternativen Angebote als attraktiv ansehen. 
Im ersten Schritt sollten Unternehmen in der Verteidigerposition danach trachten, den Erwartungen ihrer Kunden gerecht zu werden. Sie sollten darauf hinarbeiten, dass Kunden durchgängig positive Erfahrungen bei Transaktionen mit ihrem Stromanbieter sammeln. In einem zweiten Schritt können sie versuchen, die Zufriedenheit ihrer Kunden zu erhöhen. Beispielsweise können sie die Kundenerwartungen übertreffen oder imagefördernde Maßnahmen durchführen. So wichtig die Zufriedenstellung der Kunden ist, so fahrlässig wäre es, sich allein auf sie als Mittel gegen Kundenabwanderung zu stützen. So stellt auch (Seilheimer ET AL., 2002, S. 84) fest, dass für den Erfolg am Markt eine Konzentration des Marketings auf den Faktor Kundenzufriedenheit nicht ausreicht.

Eine weitere mögliche Schlussfolgerung aus den Ergebnissen dieser Arbeit ist, dass Unternehmen in der Verteidigerposition zahlreiche Wechselbarrieren aufbauen sollten, sodass für Kunden ein Anbieterwechsel mit hohen Wechselkosten einhergeht. Auf diese Weise könnten insbesondere unzufriedene Kunden gehalten werden. Diese Empfehlung ist gerade dann sinnvoll, wenn Unternehmen gewöhnlich ihre Kunden zufrieden stellen, aber trotzdem eine Art „Versicherung“ gegen Kundenabwanderung für den zwar seltenen, aber womöglich nicht vollständig vermeidbaren Fall haben möchten, dass ihnen Fehler bei der Leistungserbringung unterlaufen. JonES ET AL. (2002, S. 441) merken an, dass „managing customer perceptions of switching costs to foster retention represents a powerful tactical element in customer loyalty programs". In die gleiche Stoßrichtung zielen BENDAPUDI und Berry (1997), Fornell (1992) sowie Guiltinan (1989). Porter (1980, S. 122) fordert dazu auf, dass , [i] n view of the potential importance of switching costs, the impact of all strategic moves on switching costs should be considered."

Wechselkosten gehen mit höheren Gewinnen für die Anbieter (BEGGS und KLEMPERER, 1992) und unelastischen Reaktionen der Abnehmer auf Preise (FARRELL und SHAPIRO, 1988) einher. Wechselkosten der Käufer verleihen dem Anbieter in gewissem Umfang monopolistische Macht; FARrell und Shapiro (1988, S. 123) konkretisieren, dass „a buyer is open to exploitation by an opportunistic seller who could raise his price above competitors' by an amount almost equal to the buyer's switching cost." Ist für Konsumenten der Kauf eines Produkts bzw. der Wechsel zu einem anderen Anbieter mit Suchkosten verbunden, lassen sich laut DiAmond (1971, S. 166) durch die Anbieter höhere Preise erzielen.

Wechselkosten können des Weiteren Markteintrittsbarrieren darstellen und zu nachhaltigen strategischen Vorteilen führen (KARAKAYA und STAHL, 1989; KERIN ET AL., 1992). Jones ET AL. (2002, S. 448) empfehlen Anbietern in der Verteidigerposition, ein aktives Wechselkostenmanagement als Teil einer Gesamtstrategie zur Kundenbindung anzuwenden. ZinnBAUER (2001, S. 251) rät, verstärkt die Sicherheit der Versorgung zu kommuni- 
zieren, mittels Imagekampagnen eine Vertrauensbasis zu schaffen und zugleich das wahrgenommene Risiko als Wechselbarriere zu nutzen. Neben dem wahrgenommenen Risiko sind auch der direkte Aufwand eines Anbieterwechsels sowie die Such- und Informationskosten von Belang. Kurzfristig ließe sich mittels einer geeigneten Kommunikationspolitik nicht nur den Kunden das Risiko eines Anbieterwechsels bewusst machen, sondern auch die Zeit und die Mühe betonen, die ein Anbieterwechsel mit sich bringt. Langfristig könnte sich in Anlehnung an den klassischen Marketing-Mix ein "Wechselkosten-Mix" von Prozessen und Taktiken dazu eignen, auf der einen Seite das Kundenwechselverhalten zu reduzieren und auf der anderen Seite zugleich potenzielle Kunden zu einem Anbieterwechsel zu ermutigen.

Wechselbarrieren sind unabhängig von der Einstellung des Kunden zu der Beziehung. Sie können sowohl bei zufriedenen als auch bei unzufriedenen Kunden eingesetzt werden. Im Falle der Kombination unzufriedener Kunde mit starker Kundenbindung durch Wechselbarrieren kann man von einer erzwungenen Beziehung bzw. einer „Fesselung“ ausgehen. Eine solche unfreiwillige Kundenbindung wendet sich gegen das Streben des Kunden nach Autonomie und sollte, wenn überhaupt, mit Vorsicht eingesetzt werden, da sie sich negativ auf die Einstellung des Kunden auswirken kann. Wechselbarrieren müssen keine gesicherte Basis für eine langfristige Geschäftsbeziehung darstellen (Diller, 1996, S. 88). Eine alleinige Konzentration auf die Schaffung von Wechselbarrieren unter Vernachlässigung der Kundenzufriedenheit ist also weniger empfehlenswert, besonders wenn die Unzufriedenheit bei Kunden nicht nur kurzzeitig, sondern dauerhaft ist und wenn die Kunden die Schuld für die hohen Wechselkosten dem Anbieter zuschreiben. In diesen Fällen werden Kunden möglicherweise bei ihrem Anbieter bleiben, sich jedoch in anbieterbezogener Sabotage betätigen, wie z. B. negativer Weiterempfehlung - ganz im Sinne der von JoneS und SAsser (1995, S. 96f.) geprägten Bezeichnung „terrorists“. Bei bewusst eingeführten Wechselbarrieren wird der Kunde diese nur akzeptieren, wenn die zukünftige Einschränkung seiner Wahlfreiheit durch andere Vorteile kompensiert wird (EGGERT, 1999, S. 51).

Im Rahmen dieser Arbeit hat sich gezeigt, dass die Attraktivität der Alternativen ein weiterer Einflussfaktor der Wechselabsicht und des Wechselvollzugs ist. Da die Attraktivität der Alternativen keine absolute Größe ist, sondern der Differenz zwischen dem eigenen Angebot und konkurrierenden Angeboten entspricht, lässt sie sich durch die Unternehmen im Markt aktiv gestalten. Bei der Gestaltung des Angebots sind zwei Aspekte zu beachten: Einerseits sollten Unternehmen in Verteidigerposition darauf achten, dass ihr eigenes Angebot in Relation zu den Konkurrenzangeboten ausreichend attraktiv ist. Andererseits können Unternehmen in der Verteidigerposition davon profitieren, dass ihre 
Kunden Wechselkosten wahrnehmen und dass die Attraktivität der Alternativen nur eine schwache Wirkung entfaltet. Es besteht daher keine Notwendigkeit, mit den Angeboten von Konkurrenten gleichzuziehen oder diese gar zu übertreffen. Für Anbieter ist es wichtig zu wissen, welche Produkteigenschaften Konsumenten ihren Produktbeurteilungen zu Grunde legen. Wenn sich - wie im Strommarkt für Haushaltsstromkunden - ein Produkt kaum differenzieren lässt, spiegelt sich die Attraktivität des Angebots fast ausschließlich im Preis wider. Die Preise, die Unternehmen in der Verteidigerposition von ihren Kunden verlangen, können durchaus höher sein als die konkurrierender Anbieter, ohne dass dies einen spürbaren Einfluss auf das Wechselverhalten der Kunden hätte. Für Produktkategorien, die sich besser zur Differenzierung eignen, können auch andere Aspekte als der Preis eine wichtige Rolle spielen.

Als wichtiger Faktor, der sowohl Einfluss auf die Wechselabsicht als auch den Wechselvollzug hat, stellte sich das Involvement heraus. Ein höheres Involvement von Konsumenten fördert das Wechselverhalten. Unternehmen in der Verteidigerposition sollten daher so handeln, dass Kunden ein geringstmögliches Involvement entwickeln. So sollten sie z. B. bei imagefördernden Maßnahmen oder Kundenbindungsprogrammen stets darauf achten, dass das Involvement nicht gesteigert wird. Die Problematik wird insbesondere bei der Werbung deutlich: Während bislang in der Werbung primär versucht wurde, das Involvement für ein bestimmtes Produkt bzw. eine Produktgruppe zu steigern, wäre dieses Vorgehen für Unternehmen in der Verteidigerposition eher schädlich. Es stellt sich daher erstmalig die folgende Frage: Wie kann man bei Konsumenten das Image eines Anbieters steigern, ohne dabei zugleich das Involvement zu erhöhen? Eine Möglichkeit bestünde darin, das jeweilige Unternehmen bspw. allgemein als sympathisch, vertrauenswürdig, zuverlässig oder verantwortlich handelnd darzustellen und dabei das angebotene Produkt vollkommen auszublenden. Als Marketinginstrument eignet sich dafür Sponsoring. Eine zweite Möglichkeit ist „Stillhalten“, d.h. das Unternehmen achtet darauf, alle Aktionen zu unterlassen, die in irgendeiner Form das Involvement seiner Kunden erhöhen könnten. Eine dritte Möglichkeit, das Involvement der Kunden niedrig zu halten, eröffnet sich Anbietern, die nicht nur Strom liefern, sondern auch auf andere Weise zur Versorgung beitragen (z. B. Gas, Wasser/Abwasser, Fernwärme, Müllabfuhr etc.). Sie können für die erbrachten Leistungen eine gebündelte Rechnung ausstellen und damit weitestgehend eine höhere Involvierung ihrer Kunden mit einem einzelnen Produkt vermeiden. Besser noch als zu vermeiden, dass das Involvement der Konsumenten steigt, wäre es für Unternehmen in der Verteidigerposition, das Involvement ihrer Kunden zu senken. Allerdings wurden bislang im Rahmen des Marketingmanagements noch keine Instrumente vorgestellt, mit denen sich dies erreichen ließe. 
Eine tiefergehende Betrachtung verdient der moderierende Einfluss des Involvements. Da ein höheres Involvement die Wirkung von Kundenzufriedenheit und Wechselkosten verstärkt, mag eine Erhöhung des Involvements auf den ersten Blick als vielversprechend erscheinen. Spätestens auf den zweiten Blick wird jedoch deutlich, warum auch der moderierende Einfluss des Involvements dafür spricht, eine Erhöhung des Involvements der Kunden zu vermeiden: Erstens verstärkt ein höheres Involvement auch die Wirkung von Unzufriedenheit, d.h. manifest unzufriedene Kunden fassen einen Anbieterwechsel eher ins Auge als latent unzufriedene. Zweitens besteht die Gefahr, dass Kunden, die sich manifesten Wechselkosten ausgesetzt sehen, eher zur Ausübung anbieterbezogener Sabotage (bspw. negative Weiterempfehlung) neigen. Drittens ist der direkte Effekt des Involvements stärker als die moderierenden Effekte.

Ausgehend von den in dieser Arbeit identifizierten Determinanten des Wechselverhaltens sind also die folgenden vier Handlungsempfehlungen erfolgversprechend für die Vermeidung von Kundenabwanderung:

- die Sicherstellung der Kundenzufriedenheit durch Treffen der Kundenerwartungen,

- die Errichtung von Wechselbarrieren, sofern sie sich nicht auf den Anbieter zurückführen lassen,

- die Justierung des Angebots, sodass konkurrierende Angebote nicht wesentlich attraktiver sind, sowie

- die Vermeidung einer Erhöhung des Kundeninvolvements.

Abb. 7.3 fasst die Strategieoptionen für Unternehmen in der Verteidigerposition zusammen.

Die gewonnenen Erkenntnisse können helfen, für verschiedene Marketinginstrumente zu prüfen, inwieweit sie Kunden vom Wechseln abhalten können. Dies lässt sich am Beispiel von Kundenbindungsprogrammen illustrieren: Kundenbindungsprogramme eignen sich in gewissem Umfang dazu, die Wechselkosten zu erhöhen, die Attraktivität der Alternativen relativ zum gegenwärtigen Angebot zu verringern oder die Kundenzufriedenheit zu steigern. Im Gegenzug erhöhen sie jedoch das Involvement, sodass der Bindungseffekt zumindest teilweise neutralisiert wird.

\subsubsection{Implikationen für Unternehmen in der Angreiferposition}

Die bestehenden Kundenbeziehungen, die auf Kundenseite ggf. von hoher Zufriedenheit, hohen Wechselkosten und niedrigem Involvement geprägt sind, stellen für angreifende 


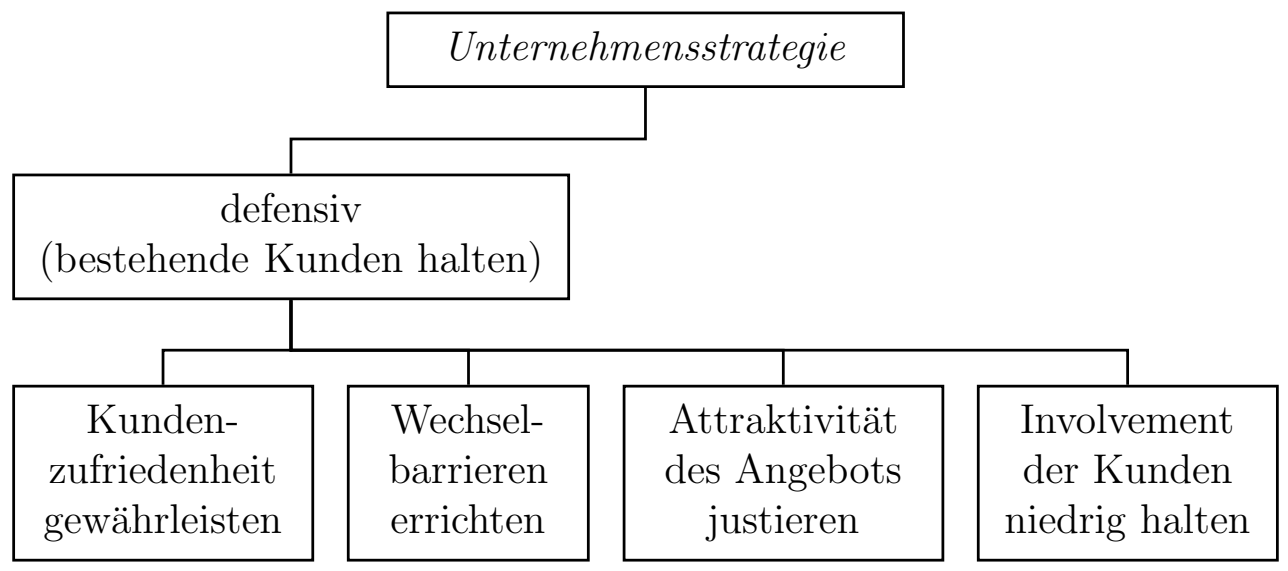

Abbildung 7.3: Strategieoptionen eines Anbieters in der Verteidigerposition

Unternehmen eine Eintrittsbarriere in den jeweiligen Markt dar (Simon und Homburg, 1995, Sp. 1748). Die in der vorliegenden Arbeit identifizierten Determinanten des Wechselverhaltens bilden die Grundlage für Handlungsoptionen, die diese Eintrittsbarriere überwinden helfen.

Ein erster Ansatzpunkt für Angreifer kann die Kundenzufriedenheit sein. Zwar besteht kaum eine Möglichkeit, die Ist-Komponente der Kundenzufriedenheit zu beeinflussen schließlich hängt die Erfüllung der Kundenerwartungen hauptsächlich davon ab, welche Erfahrungen Kunden mit ihrem Anbieter machen. Jedoch lässt sich die Soll-Komponente der Kundenzufriedenheit, also die Erwartungen bzw. das als gerecht Empfundene, durch Marketinginstrumente beeinflussen. Dies kann letztendlich dazu führen, dass Konsumenten mit ihrem aktuellen Anbieter unzufrieden werden. Durch eine ausgesuchte Kommunikationspolitik lassen sich bspw. die Ankerpunkte der Konsumenten verschieben, sodass die Kundenzufriedenheit mit dem etablierten Anbieter sinkt.

Ein zweiter Ansatzpunkt sind die wahrgenommenen Wechselkosten. Anbieter in der Angreiferposition können hierzu die Ursachen von Wechselkosten bekämpfen oder die Wechselkosten kompensieren. Auf einige Ursachen von Wechselkosten können Angreifer unmittelbar einwirken. Andere Ursachen von Wechselkosten lassen sich nur auf höherer Ebene abstellen, d.h. man muss auf die Gesetzgebung bzw. die Regulierung Einfluss nehmen. Im Rahmen der Preispolitik lassen sich Kompensationszahlungen für erlittene oder zu erleidende Wechselkosten vorsehen. Eine geeignete Distributionspolitik kann dazu beitragen, die wahrgenommenen Wechselkosten zu reduzieren. Sie sollte so ausgelegt sein, dass 
Konsumenten ein Anbieterwechsel so einfach wie möglich gemacht wird. Auch die Kommunikationspolitik kann zur Reduzierung der Wechselkosten eingesetzt werden. Durch geeignete Information lassen sich sowohl die Informations- und Suchkosten von Konsumenten als auch das wahrgenommene Risiko eines Anbieterwechsels reduzieren. Zudem kann Information dazu beitragen, dass die direkten Wechselkosten als niedriger empfunden werden.

Ein dritter Ansatzpunkt ist die Attraktivität des Angebots. Da die Attraktivität des Angebots relativ zum Angebot der etablierten Anbieter zu sehen ist, bieten sich zwei wesentliche Gestaltungsmöglichkeiten: erstens das eigene Wertangebot, zweitens der dafür verlangte Preis. Ein überlegenes Wertangebot lässt sich z. B. durch Produktaugmentierung erreichen, also die Ausstattung von Produkten mit Zusatznutzen. Produktaugmentierung stellt zugleich eine Möglichkeit dar, den Ankerpunkt von Konsumenten zu verschieben, d. h. die Kundenerwartungen an ein Produkt und damit mittelbar auch die Kundenzufriedenheit mit dem aktuellen Anbieter zu verändern. Gerade bei homogenen Produkten wie bspw. Haushaltsstrom spielt der Preis die maßgebliche Rolle beim Vergleich der Attraktivität unterschiedlicher Angebote. Unternehmen in der Angreiferposition müssen danach trachten, Konsumenten durch deutlich niedrigere Preise für sich zu gewinnen. Da die Unternehmen, die in der Verteidigerposition sind, so gut wie gar nicht zwischen neuen und existierenden Kunden diskriminieren können, ist eher nicht davon auszugehen, dass die etablierten Anbieter ihre Preise auf das niedrigere Niveau der Angreifer senken (FARRELL und Shapiro, 1988, S. 124). Fudenberg und Tirole (1984, S. 361) sprechen in diesem Zusammenhang vom sog. „fat-cat effect" und verstehen darunter einen aufgrund zu großer Investitionen reduzierten Ansporn, auf Wettbewerber aggressiv zu reagieren. Er bewirkt, dass etablierte Anbieter mit großen Marktanteilen höhere Preise fordern als ihre schlankeren Rivalen und auf diese Weise Marktanteile verlieren.

Ein vierter Ansatzpunkt ist das Involvement. Es erwies sich im Rahmen der Empirie als wichtige Determinante sowohl der Wechselabsicht als auch des Wechselvollzugs. Die Begründung liegt auf der Hand: Konsumenten, die sich nicht mit der Versorgungsbeziehung befassen, werden kaum ihren Anbieter wechseln. Das Involvement lässt sich als notwendige Bedingung für einen Anbieterwechsel auffassen. Da das Involvement der Konsumenten nicht anbieter-, sondern produktbezogen ist und ein hohes Involvement für alle Anbieter in der Angreiferposition förderlich ist, empfiehlt sich die Bildung von Allianzen: Anbieter in der Angreiferposition könnten gemeinschaftlich, z. B. durch Verbände organisiert, auf eine Erhöhung des Involvements der Konsumenten hinarbeiten.

Abb. 7.4 fasst die Handlungsempfehlungen für Unternehmen in der Angreiferposition zusammen. 


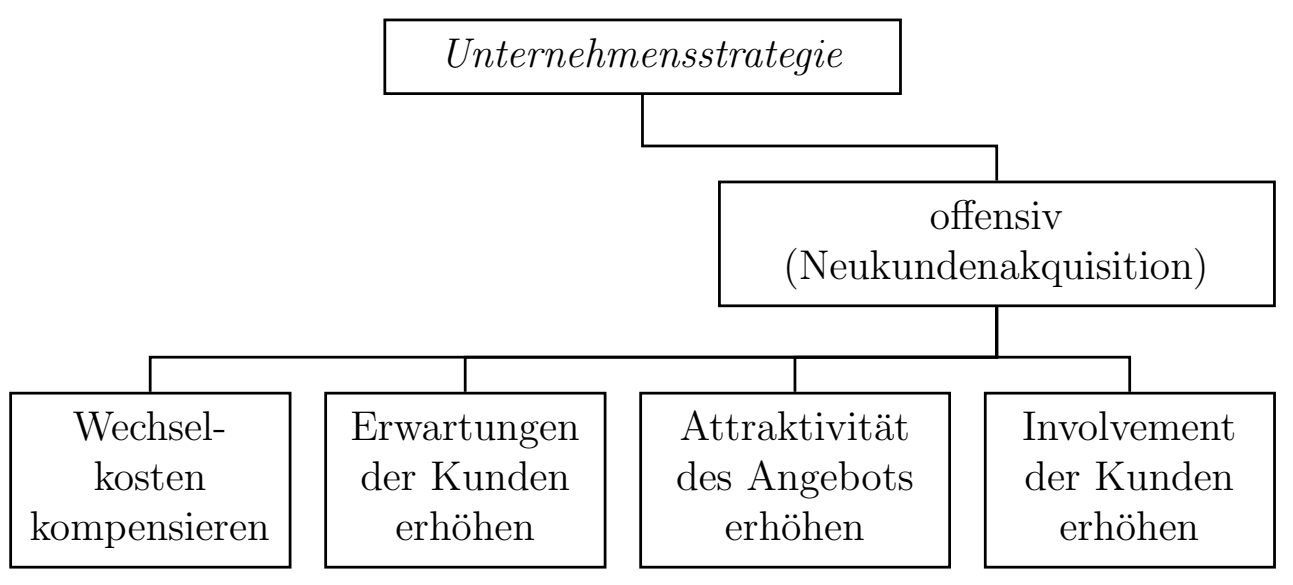

Abbildung 7.4: Strategieoptionen eines Anbieters in der Angreiferposition

\subsection{Grenzen der Arbeit und Anknüpfungspunkte für weitere Forschung}

Für diese Arbeit gelten die allgemeinen Einschränkungen bzgl. der Interpretation von Strukturgleichungsmodellen. $\mathrm{Zu}$ beachten ist insbesondere, dass der Forschungsaufbau zwar erlaubt, gerichtete Beziehungen zwischen den interessierenden Faktoren aufzuzeigen, jedoch keine Aussagen zu Kausalbeziehungen zulässt. Die Untersuchung von Kausalitäten in Bezug auf die hypothetischen Wirkbeziehungen lässt sich am ehesten durch Experimente über Partialmodelle durchführen.

Die empirische Überprüfung der moderierenden Effekte konnte die sechs Hypothesen $\mathrm{H}_{6}$, $\mathrm{H}_{10}, \mathrm{H}_{15}, \mathrm{H}_{16}, \mathrm{H}_{17}$ und $\mathrm{H}_{18}$ stützen. Dies bedeutet, die Attraktivität der Alternativen und die Wechselkosten moderieren die Wirkbeziehung zwischen Kundenzufriedenheit und Wechselvollzug, und das Involvement sorgt für unterschiedliche Wirkstärken von latenter und manifester Kundenzufriedenheit sowie latenten und manifesten Wechselkosten, jeweils sowohl auf die Wechselabsicht als auch auf den Wechselvollzug. Chaplin (1997, S. 875) weist treffend darauf hin, dass statistisch gesehen keine Unterscheidung zwischen Prädiktor- und Moderatorvariable vorgenommen wird, da die multiplikative Verknüpfung im Rahmen der statistischen Analyse kommutativ ist. Er folgert daraus, dass „[i]t is equally legitimate (statistically) to refer to the first independent variable as a moderator of the relation between the second independent variable and the dependent variable." Empirisch würden also die folgenden alternativen Hypothesen dieselbe Unterstützung erfahren: 
- $\mathrm{H}_{6}^{*}$ : Je höher die Kundenzufriedenheit, desto weniger Einfluss hat die Attraktivität der Alternativen auf den Wechselvollzug.

- $\mathrm{H}_{10}^{*}$ : Je höher die Kundenzufriedenheit, desto weniger Einfluss haben Wechselkosten auf den Wechselvollzug.

- $\mathrm{H}_{15}^{*}$ : Je höher die Kundenzufriedenheit, desto weniger Einfluss hat das Involvement auf die Wechselabsicht.

- $\mathrm{H}_{16}^{*}$ : Je höher die Kundenzufriedenheit, desto weniger Einfluss hat das Involvement auf den Wechselvollzug.

- $\mathrm{H}_{17}^{*}$ : Je höher die Wechselkosten, desto weniger Einfluss hat das Involvement auf die Wechselabsicht.

- $\mathrm{H}_{18}^{*}$ : Je höher die Wechselkosten, desto weniger Einfluss hat das Involvement auf den Wechselvollzug.

Aufgabe der Forschung bleibt es, diese rein induktiv getriebenen neuen Hypothesen konzeptionell zu durchdringen.

Ein weiteres wichtiges Ergebnis dieser Arbeit ist, dass die hypothetischen Einflussfaktoren auf den Wechselvollzug in Art und Umfang nicht mit den Einflussfaktoren auf die Wechselabsicht übereinstimmen. Für eine tiefergehende Untersuchung des Phänomens des Wechselverhalten können an dieser Stelle Längsschnittanalysen hilfreich sein. Durch sie ließen sich Einstellungen und Verhalten von Konsumenten über einen längeren Zeitraum hinweg beobachten. Obwohl Längsschnittstudien, wie bspw. die Arbeit von CRosBy und Stephens (1987), sehr zum Erkenntnisgewinn beitragen, werden sie aufgrund des dafür notwendigen Forschungsaufwands nur selten durchgeführt.

Die aufgestellten Hypothesen wurden in einem konkreten Markt, dem Strommarkt für Haushaltsstromkunden, überprüft. Trotz der vielen Gemeinsamkeiten, die die Beziehungen zwischen Konsumenten und Stromanbietern mit anderen Versorgungsbeziehungen haben, sollten die Ergebnisse der empirischen Untersuchung nicht unreflektiert auf andere Branchen verallgemeinert werden. Stattdessen ist hier die Durchführung von Replikationsstudien angezeigt, um die gewonnenen Erkenntnisse für andere Branchen zu adaptieren. Dies gilt in besonderem Maße für die direkten Wirkungen des Involvements, da sich aus der Literatur gegensätzliche Hypothesen ableiten ließen.

Unter dem Gesichtspunkt, dass diese Arbeit zu den ersten zählt, die das Wechselverhalten bei Versorgungsbeziehungen untersuchen, ist es möglich, dass noch andere Variablen existieren, die in einem Modell des Wechselverhaltens Berücksichtigung finden könnten. 
Der Anteil der erklärten Varianz bei der Wechselabsicht und insbesondere beim Wechselvollzug lässt vermuten, dass noch andere Faktoren eine Rolle spielen. Denkbar in diesem Zusammenhang wären z. B. Faktoren wie die Beziehung zum Anbieter (CROSBY und StEPHENS, 1987) oder das Suchen nach Abwechslung („variety-seeking“, vgl. MCALISTER und Pessemier, 1982).

Das vorliegende Modell ist auch insofern beschränkt, als dass es Dynamik, die möglicherweise im Wechselprozess auftritt, nicht berücksichtigt. So mag es z. B. Individuen geben, die ihren Anbieter nicht wechseln, obwohl sie hoch involviert sind, mit ihrem derzeitigen Anbieter eher unzufrieden sind und die Wechselkosten als gering oder sogar deutlich niedriger als die erzielbaren Einsparungen empfinden. Ursächlich für dieses Phänomen ist möglicherweise die Hoffnung dieses Individuums, ein noch besseres Angebot eines anderen Anbieters durch eine Ausweitung der Suche nach alternativen Anbietern oder durch dynamische Marktveränderungen in der nahen Zukunft zu erhalten. In diesem Fall möchte das Individuum mit einem Anbieterwechsel warten, bis sich ein noch besseres Angebot auftut, damit die mit einem Anbieterwechsel verbundenen Wechselkosten nicht mehrmals anfallen. Im Prinzip erkauft sich das Individuum die Freiheit, zu einem noch günstigeren als dem bisher als besten bekannten Anbieter zu wechseln, indem die Wechselkosten eines möglichen zweiten Wechsels in den Wechselkosten des ersten Wechsels teilweise antizipiert werden. Ausgehend von dieser Überlegung unterbreiten ScHLESINGER und SchulenBurg (1995, S. 612) einen Vorschlag zur Modellierung dieses Phänomens: Die Wechselkosten ließen sich erweitern um eine Art Option für die Verzögerung eines Anbieterwechsels. Die Funktionsweise dieser Option ist mit Wertpapieroptionen in der LongPosition vergleichbar: Der Kunde hat das Recht, aber nicht die Pflicht, seinen Anbieter zu wechseln. Der Wert dieser Option entspricht dem Nutzen aus der Flexibilität, auch später immer noch wechseln zu können.

Die vorliegende Arbeit identifiziert vier Ansatzpunkte, durch die Unternehmen das Wechselverhalten von Konsumenten beeinflussen können: die Kundenzufriedenheit, die wahrgenommenen Wechselkosten, die Attraktivität der Alternativen sowie das Involvement der Kunden. Um diese Ansatzpunkte für das Marketingmanagement handhabbar zu machen, sind auf Unternehmensebene die verfügbaren Instrumentarien auf ihre Wirksamkeit hinsichtlich der vier Ansatzpunkte zu untersuchen. Hierfür lassen sich keine für sämtliche Unternehmen gültige Empfehlungen geben, vielmehr muss jedes Unternehmen individuell analysieren, inwieweit sich verfügbare Marketinginstrumente effektiv und effizient einsetzen lassen.

Eine solche Analyse wurde exemplarisch für einen Anbieter durchgeführt. Hierbei wurde anhand eines Teils der Kunden versucht, das Zustandekommen von Kundenzufriedenheit 


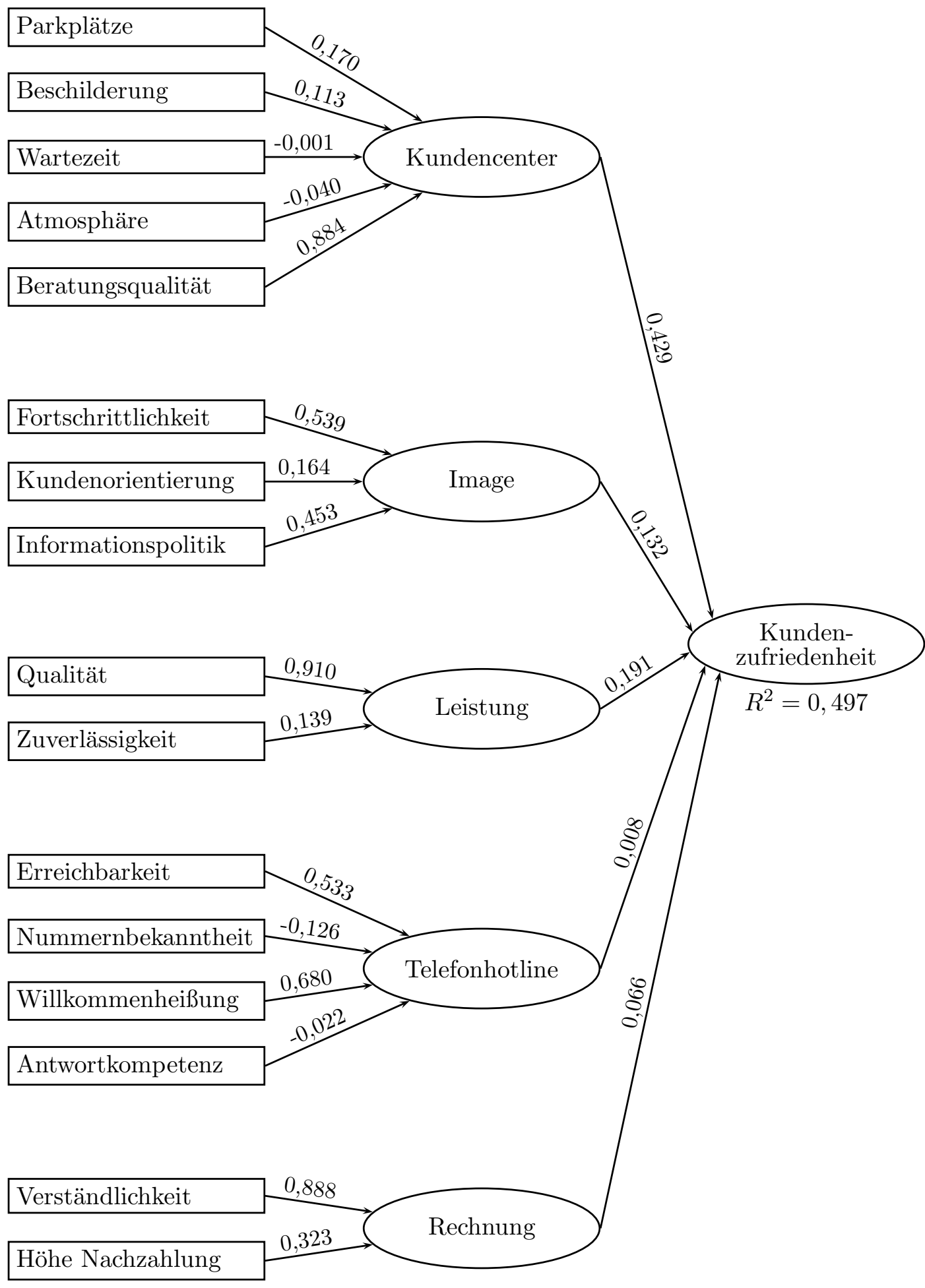

Abbildung 7.5: Auswirkung von Marketinginstrumenten auf die Kundenzufriedenheit (nicht-repräsentative Illustration am Beispiel eines regionalen Stromanbieters) 
zu erklären. Fünf potenzielle Einflussfaktoren und jeweils zugehörige Instrumente wurden danach untersucht, inwieweit sie zur Bildung von Kundenzufriedenheit beitragen:

- das Kundencenter als Hauptbegegnungsstätte zwischen Unternehmen und Kunden,

- das Image des Unternehmens,

- die vom Unternehmen erbrachte Leistung,

- die Telefonhotline als zweite Kontaktmöglichkeit sowie

- die Rechnung, die die Kunden jährlich erhalten.

Abb. 7.5 zeigt das Ergebnis dieser unternehmensspezifischen Analyse. Über 49,7\% der Varianz der Kundenzufriedenheit lässt sich im vorliegenden Fall durch die einzelnen Einflussfaktoren erklären. Als dominant erweist sich das Kundencenter, und hier insbesondere die Qualität der Beratung. Wichtig bleibt festzuhalten, dass diese Ergebnisse allein für den betrachteten Anbieter Gültigkeit besitzen; eine Verallgemeinerung ist nicht möglich. 


\section{Zusammenfassung}

Seit der Strommarktliberalisierung im Jahr 1998 können Haushaltsstromkunden ihren Stromanbieter wechseln. Anders als vielfach erwartet, hat bislang nur ein geringer Teil der Konsumenten den Anbieter gewechselt. Die Marketingwissenschaft ließ bisher die Frage unbeantwortet, welche Faktoren die Konsumenten veranlassen, bei ihrem Anbieter zu bleiben bzw. ihren Anbieter zu wechseln. Des Weiteren stellte sich die Frage, warum viele Konsumenten, die die Absicht zum Wechsel haben, den Wechsel nicht vollziehen.

Kundenbeziehungen, die vom Charakter her der Beziehung zwischen Konsument und Stromanbieter ähneln, sind die sog. Versorgungsbeziehungen. Empirische Arbeiten zu den Determinanten von Kundenloyalität und Wechselverhalten bei Versorgungsbeziehungen legen nahe, dass primär die Kundenzufriedenheit und in zweiter Linie die Wechselkosten einen entscheidenden Erklärungsbeitrag liefern. Die Diskrepanz zwischen bekundetem und realisiertem Wechselverhalten kann auf unvorhergesehene Umwelteinflüsse, neue Information, die Zwischenzeit, Unterschiede in der Konkretheit, einen unvorhergesehenen situativen Kontext, den Grad der Willenskontrolle sowie die Instabilität der Absichten zurückzuführen sein. Eine besonders in der Praxis beliebte Begründung für die geringe Wechselrate, nämlich die Trägheit der Konsumenten, ist - soll es sich nicht um eine Tautologie handeln - eine Umschreibung für hohe wahrgenommene Wechselkosten oder ein $\mathrm{zu}$ niedriges Involvement.

Als theoretische Grundlagen zur Erklärung der Fortsetzung von Versorgungsbeziehungen diente zum einen die Lerntheorie der instrumentellen Konditionierung, die die Fortsetzung von Beziehungen auf die Zufriedenheit mit der aktuellen Situation zurückführt, und zum anderen ein Konzept von BliEMEL und EGGERT (1998b), nach dem sich Motivationen zur Fortsetzung von Beziehungen anhand der beiden Dimensionen Wille und Zwang zur Fortsetzung kategorisieren lassen. Als theoretische Grundlagen zur Erklärung der Beendigung von Versorgungsbeziehungen wurden vier Theorien vorgestellt: Gemäß der Theorie der kognitiven Dissonanz trachten Menschen danach, kognitive Dissonanzen zu vermeiden, d. h. ihr psychisches Gleichgewicht beizubehalten. Aus der Theorie der kognitiven Dissonanz lässt sich ableiten, dass zufriedene Konsumenten bei ihrem Anbieter bleiben, während unzufriedene Konsumenten ihr Erwartungssniveau senken oder ihren Anbieter wechseln. Mit der Theorie überlegten Handelns lässt sich willentliches Verhalten, also insbesondere 
auch Wechselverhalten, modellieren. Aus ihr entwickelte sich die Theorie des geplanten Verhaltens, die sich nicht nur auf willentliches Verhalten beschränkt, sondern auch das Zustandekommen anderer Verhaltensarten zu erklären vermag. Die Austauschtheorie führt die Fortsetzung oder Beendigung von Beziehungen auf die Erfüllung individueller Vergleichsstandards zurück.

Aufbauend auf den Ergebnissen der bestehenden empirischen Studien zu Versorgungsbeziehungen und den vorgestellten Theorien wurden vier potenziell maßgebliche Determinanten des Wechselverhaltens identifiziert: die Kundenzufriedenheit, die Attraktivität der Alternativen, die wahrgenommenen Wechselkosten sowie das Involvement. Jedes der vier Konstrukte wurde zunächst konzeptualisiert, bevor seine möglichen direkten und moderierenden Wirkungen auf die Wechselabsicht und den Wechselvollzug als Hypothesen formuliert wurden. Auf diese Weise wurden 20 Hypothesen gewonnen und anschließend zu einem Hypothesensystem zusammengefügt.

Als statistisches Analysewerkzeug für die quantitative Analyse des Hypothesensystems gelangte die PLS-Pfadmodellierung zum Einsatz. Mangels bestehender einführender Literatur wurde ein Leitfaden für die PLS-Pfadmodellierung erstellt. Im Zuge dessen wurde eine neue Methode zur Schätzung von Interaktionseffekten eingeführt.

Eine telefonische Befragung unter den Kunden zweier regionaler Stromanbieter lieferte die Daten für die empirische Analyse. Nach der Bestätigung einer reliablen und validen Messung der latenten Variablen Kundenzufriedenheit, Wechselkosten, Attraktivität der Alternativen, Involvement und Wechselabsicht führte die Analyse der direkten Wirkbeziehungen zu folgenden Ergebnissen: In erster Linie determinieren die Kundenzufriedenheit und das Involvement die Wechselabsicht, in zweiter Linie die wahrgenommenen Wechselkosten und die Attraktivität der Alternativen. Der Wechselvollzug hängt in erster Linie von den wahrgenommenen Wechselkosten sowie dem Involvement ab, in zweiter Linie von der Kundenzufriedenheit und der Attraktivität der Alternativen. Die Wirkweise der Wechselkosten erklärt, warum viele Konsumenten, die die Absicht zum Anbieterwechsel gebildet haben, vom Wechselvollzug ablassen: Sie beziehen die Wechselkosten noch nicht in vollem Umfang in ihre Überlegungen ein, wenn sie die Wechselabsicht bilden, wohl aber, wenn es um den tatsächlichen Wechselvollzug geht. Zusätzlich zu den direkten Effekten lassen sich einige moderierende Effekte nachweisen: Manifeste Kundenzufriedenheit und manifeste wahrgenommene Wechselkosten haben eine stärkere Wirkung auf die Wechselabsicht und den Wechselvollzug als latente Kundenzufriedenheit bzw. Wechselkosten. Darüber hinaus wird die Wirkung der Kundenzufriedenheit auf den Wechselvollzug nicht nur durch das Involvement, sondern auch durch die wahrgenommenen Wechselkosten und die Attraktivität der Alternativen moderiert. 
Ausgehend von den vier identifizierten Determinanten des Wechselverhaltens - der Kundenzufriedenheit, den wahrgenommenen Wechselkosten, der Attraktivität der Alternativen sowie dem Involvement - wurden in einem ersten Schritt Implikationen für die Marketingwissenschaft herausgearbeitet. Insbesondere wurde eine Erweiterung der Austauschtheorie durch den moderierenden Einfluss des Involvements diskutiert. In einem zweiten Schritt wurden Handlungsempfehlungen für Unternehmen gegeben: Anbieter in der Verteidigerposition können Einfluss auf sämtliche identifizierten Determinanten des Wechselverhaltens nehmen. Sie sollten Kundenunzufriedenheit vermeiden, die Attraktivität ihres Angebots an Konkurrenzangeboten orientieren, Wechselbarrieren errichten, um Wechselkosten zu induzieren, sowie das Involvement ihrer Kunden niedrig halten. Auch Anbietern in der Angreiferposition steht die Beeinflussung aller identifizierten Determinanten des Wechselverhaltens offen. Im Gegensatz zu Unternehmen in der Verteidigerposition sollten sie Kundenerwartungen erhöhen, um Kundenunzufriedenheit mit der bestehenden Versorgungsbeziehung hervorzurufen, die Wechselbarrieren neutralisieren, um so die Wechselkosten der Konsumenten zu reduzieren, ihr Angebot deutlich attraktiver als die Angebote der etablierten Anbieter gestalten sowie das Involvement der Konsumenten erhöhen. 


\section{Literaturverzeichnis}

Ajzen, ICEK (1985). From Intentions to Actions. A Theory of Planned Behavior. In: Kuhl, Julius; Beckmann, Jürgen (Eds.): Action control. From Cognition to Behavior. Berlin/New York: Springer, pp. 11-39.

Ajzen, ICEK (1989). Attitude Structure and Behavior. In: Pratkanis, Anthony R.; Breckler, Steven J.; Greenwald, Anthony G. (Eds.): Attitude Structure and Function. Hillsdale, NJ: Lawrence Erlbaum Associates, pp. 241-274.

Ajzen, Icek; Brown, Thomas C.; Carvajal, Franklin (2004). Explaining the Discrepancy Between Intentions and Actions: The Case of Hypothetical Bias in Contingent Valuation. Personality and Social Psychology Bulletin, 30 (9), pp. 1108-1121.

Ajzen, ICEK; Fishbein, Martin (1980). Understanding Attitudes and Predicting Social Behavior. Englewood Cliffs, NJ: Prentice-Hall.

Ajzen, ICeK; Madden, Thomas J. (1986). Prediction of Goal-Directed Behavior: Attitudes, Intentions, and Perceived Behavioral Control. Journal of Experimental Social Psychology, 22, pp. 453-474.

Albers, Sönke (2004). Formative versus reflektive Messmodelle. In: BliEMEL, FrieDhelm W.; Eggert, Andreas; Fassott, Georg; Henseler, Jörg (Hrsg.): Strukturgleichungsmodelle mit latenten Variablen. Der PLS-Ansatz. Workshop am 30. April 2004. Kaiserslautern: Technische Universität Kaiserslautern.

Andersen, Poul Houman (2002). A Foot in the Door. Relationship Marketing Efforts Towards Transaction-Oriented Customers. Journal of Market-Focused Management, 5, pp. 91-108.

Anderson, Eugene; Sullivan, Mary W. (1993). The Antecedents and Consequences of Customer Satisfaction for Firms. Marketing Science, 12 (2), pp. 125-143.

Anderson, Eugene W. (1994). Cross-Category Variation in Customer Satisfaction and Retention. Marketing Letters, 5 (1), pp. 19-30.

Anderson, Eugene W.; Fornell, Claes (1994). A Customer Satisfaction Research Prospectus. In: Rust, Roland T.; Oliver, Richard L. (Eds.): Service Quality. New Directions in Theory and Practice. Thousand Oaks et al.: Sage, pp. 241-268.

Anderson, Eugene W.; Fornell, Claes; Lehmann, Donald R. (1994). Customer Satisfaction, Market Share, and Profitability. Findings from Sweden. Journal of Marketing, 58 (3), pp. 53-66.

Anderson, James C.; Gerbing, David (1982). Some Methods for Respecifying Measurement Models to Obtain Unidimensional Construct Measurement. Journal of Marketing Research, 19 (4), pp. 453-460.

Anderson, James C.; Narus, James A. (1984). A Model of the Distributor's Per- 
spective of Distributor-Manufacturer Working Relationships. Journal of Marketing, 48 (Fall), pp. 62-74.

Anderson, James C.; Narus, James A. (1990). A Model of Distributor Firm and Manufacturer Firm Working Partnerships. Journal of Marketing, 54 (1), pp. 42-58.

Andreasen, Alan R. (1985). Consumer Responses to Dissatisfaction in Loose Monopolies. Journal of Consumer Research, 12 (September), pp. 135-141.

Arkes, Hal R.; Kung, Yi-Han; Hutzel, Laura (2002). Regret, Valuation and Inaction Inertia. Organizational Behavior and Human Decision Processes, 87 (2), pp. 371-385.

Assael, Henry (1992). Consumer Behavior and Marketing Action. Boston, MA: PWS-KENT, $4^{\text {th }}$ edition.

Backhaus, Klaus; Aufderheide, Detlef; Späth, Georg-Michael (1994). Marketing für Systemtechnologien. Entwicklung eines theoretisch-ökonomisch begründeten Geschäftstypenansatzes. Stuttgart: Schäffer-Poeschel Verlag.

Backhaus, Klaus; Erichson, Bernd; Plinke, Wulff; Weiber, Rolf (2003). Multivariate Analysemethoden. Eine anwendungsorientierte Einführung. Berlin et al.: Springer, 10. Auflage.

Bagozzi, Richard P. (1975). Marketing as Exchange. Journal of Marketing, 39 (3), pp. 32-39.

Bagozzi, Richard P. (1981). Evaluating Structural Equation Models with Unobservable Variables and Measurement Error. A Comment. Journal of Marketing Research, 18 (3), pp. 375-381.

Bagozzi, Richard P.; Fornell, Claes (1982). Theoretical Concepts, Measurement and Meaning. In: Fornell, Claes (Ed.): A Second Generation of Multivariate Analysis. Measurement and Evaluation. Volume II. New York: Praeger, pp. 24-38.

BAKAY, Zoltan (2003). Kundenbindung von Haushaltsstromkunden. Wiesbaden: Deutscher Universitäts-Verlag.

BalderJahn, Ingo (1986). The Robustness of LISREL Unweighted Least Squares Estimations against Small Sample Size in Confirmatory Factor Analysis. In: Gaul, Wolfgang; Schader, Martin (Eds.): Classification as a Tool of Research. Amsterdam: North Holland, pp. 3-10.

Bansal, Harvir S.; Taylor, Shirley F. (1999). The Service Provider Switching Model (SPSM). A Model of Consumer Switching Behaviour in the Service Industry. Journal of Service Research, 2 (2), pp. 200-218.

Barclay, Donald; Higgins, Christopher; Thompson, Ronald (1995). The Partial Least Squares (PLS) Approach to Causal Modeling. Personal Computer Adoption and Use as an Illustration. Technology Studies, 2 (2), pp. 285-314.

Baron, Reuben M.; Kenny, David A. (1986). The Moderator-Mediator Variable Distinction in Social Psychological Research. Conceptual, Strategic, and Statistical Considerations. Journal of Personality and Social Psychology, 51 (6), pp. 1173-1182.

Bauer, Raymond A. (1960). Consumer Behavior as Risk Taking. In: Hancok, Robert S. (Ed.): Dynamic Marketing for a Changing World. Proceedings of the $43^{\text {rd }}$ National Conference of the American Marketing Association, pp. 389-398. Chicago. 
BAYón, Tomás (1997). Neuere Mikroökonomie und Marketing. Eine wissenschaftstheoretisch geleitete Analyse. Wiesbaden: Gabler.

Bearden, William O.; Teel, Jesse E. (1983). Selected Determinants of Consumer Satisfaction and Complaint Reports. Journal of Marketing Research, 20 (1), pp. 21-28.

Beatty, Sharon E.; Homer, Pamela; Kahle, Lynn R. (1988). The InvolvementCommitment Model. Theory and Implications. Journal of Business Research, 16 (2), pp. 149-167.

Beatty, Sharon E.; Smith, Scott M. (1987). External Search Effort. An Investigation across Several Product Categories. Journal of Consumer Research, 13 (1), pp. 83-95.

Beerli, Asunción; Martín, Josefa D.; Quintana, Agustín (2004). A Model of Customer Loyalty in the Retail Banking Market. European Journal of Marketing, $38(1 / 2)$, pp. 253-275.

Beggs, Alan; Klemperer, Paul D. (1992). Multi-Period Competition With Switching Costs. Econometrica, 60 (3), pp. 651-666.

Belk, Russell W. (1985). Issues in the Intention-Behavior Discrepancy. In: SHeth, Jagdish N. (Ed.): Research in Consumer Behavior, volume 1. Greenwich, CT: JAI Press, pp. 1-33.

Belz, Christian (1994). Management von Geschäftsbeziehungen. Konzepte, Integrierte Ansätze, Anwendungen in der Praxis. St. Gallen/Wien: Thexis/Ueberreuter.

Bendapudi, NeEli; Berry, LeOnARd L. (1997). Customer's Motivations for Maintaining Relationships with Service Providers. Journal of Retailing, 73 (1), pp. 19-37.

Berry, Leonard L. (1980). Services Marketing is Different. Business, 30 (3), pp. $24-29$.

Berry, LeOnARD L. (1995). Relationship Marketing of Services. Growing Interest, Emerging Perspectives. Journal of the Academy of Marketing Science, 23 (4), pp. $236-245$.

Betzin, Jörg (2004). Die Funktionsweise des PLS-Algorithmus. In: BliEMEL, FrIEDhelm W.; Eggert, Andreas; Fassott, Georg; Henseler, Jörg (Hrsg.): Strukturgleichungsmodelle mit latenten Variablen. Der PLS-Ansatz. Workshop am 30. April 2004. Kaiserslautern: Technische Universität Kaiserslautern.

Beutin, Nikolas (2003). Verfahren zur Messung der Kundenzufriedenheit im Überblick. In: Homburg, Christian (Hrsg.): Kundenzufriedenheit. Konzepte, Methoden, Erfahrungen. Wiesbaden: Gabler, 5. Auflage, S. 115-151.

Beutin, Nikolas; Paul, Andreas; Schröder, NAdine (2001). Marketing in Energieversorgungsunternehmen Instrumente und Erfolgsfaktoren in Zeiten der Deregulierung. Zeitschrift für Energiewirtschaft, 25 (3), S. 171-188.

Beutin, Nikolas; Werner, Harald (1999). Kundenbindung in Märkten im Umbruch. Das Beispiel der Energieversorgung. In: Bruhn, Manfred; Homburg, Christian (Hrsg.): Handbuch Kundenbindungsmanagement. Wiesbaden: Gabler, S. 545-567.

Bitner, MARY Jo (1990). Evaluating Service Encounters. The Effects of Physical Surroundings and Employee Responses. Journal of Marketing, 54 (April), pp. 69-82.

Bitner, Mary Jo; Booms, Bernard H.; Tetreault, Mary Stanfield (1989). Abstract - Critical Incidents in Service Encounters. In: Bitner, Mary Jo; Crosby, 
LaWrence A. (Eds.): Designing a Winning Service Strategy. Chicago: American Marketing Association, pp. 98-99.

Bitner, Mary Jo; Booms, Bernard H.; Tetreault, Mary Stanfield (1990). The Service Encounter: Diagnosing Favorable and Unfavorable Incidents. Journal of Marketing, 54 (1), pp. 71-84.

Bitner, Mary Jo; Hubbert, Amy R. (1994). Encounter Satisfaction Versus Overall Satisfaction Versus Quality. The Customer's Voice. In: Rust, Roland T.; Oliver, RIchard L. (Eds.): Service Quality. New Directions in Theory and Practice. Thousand Oaks et al.: Sage, pp. 72-94.

Bitner, Mary Jo; Nyquist, Jody D.; Booms, Bernard H. (1985). The Critical Incident as a Technique for Analyzing the Service Encounter. In: Bloch, Thomas M.; Upah, Gregory D.; Zeithaml, Valarie A. (Eds.): Services Marketing in a Changing Environment. Chicago: American Marketing Association, pp. 48-51.

Blackwell, Roger D.; Miniard, Paul W.; Engel, James F. (2001). Consumer Behavior. Fort Worth et al.: Dryden, $9^{\text {th }}$ edition.

Bliemel, Friedhelm W.; Eggert, Andreas (1997). Exploring the Limits of Relationship Marketing: An Empirical Investigation into the Concept and Antecedents of Opportunity Mindedness (COM). In: Gummesson, Evert (Ed.): Proceedings of the $8^{\text {th }}$ International Colloquium in Relationship Marketing. Return on Relationships (ROR) and Customer Relationship Management (CRM). Stockholm: Stockholm University School of Business.

Bliemel, Friedhelm W.; Eggert, Andreas (1998a). Kundenbindung aus Kundensicht. Kaiserslauterer Schriftenreihe Marketing, Heft 4.

Bliemel, Friedhelm W.; Eggert, Andreas (1998b). Why do they keep coming back? Customer-Retention and Barriers to Change from the Customer's Perspective. In: Halinen-Kaila, Aino; Nummela, Nima (Eds.): Proceedings of the $14^{\text {th }}$ IMP Annual Conference, pp. 53-68. Turku: Turku School of Economics and Business Administration.

Bloch, Peter H.; Richins, Marsha L. (1983). A Theoretical Model for the Study of Product Importance. Journal of Marketing, 47 (Summer), pp. 69-81.

Bloemer, José M. M.; Kasper, Hans D. P. (1994). The Impact of Satisfaction on Brand Loyalty. Urging on Classifying Satisfaction and Brand Loyalty. Journal of Consumer Satisfaction, Dissatisfaction and Complaining Behavior, 7, pp. 152-160.

Bloemer, José M. M.; Kasper, Hans D. P. (1995). The Complex Relationship between Customer Satisfaction and Brand Loyalty. Journal of Economic Psychology, 16, pp. 311-329.

Bloemer, José M. M.; Kasper, Hans D. P. (1999). The Effects of Satisfaction and Involvement on Loyalty. In: Proceedings of the $28^{\text {th }}$ EMAC Conference. Berlin. CD-ROM.

Bloemer, José M. M.; Pauwels, Koen; Kasper, Hans D. P. (1996). There is More to Loyalty Than Just Satisfaction. The Effects of Satisfaction and Involvement on Brand Loyalty and Dealer Loyalty. Arbeitspapier, Universität Limburg, Belgien.

Bloemer, José M. M.; Poiesz, Theo B. C. (1989). The Illusion of Consumer Satisfaction. Journal of Consumer Satisfaction, Dissatisfaction and Complaining Behaviour, 2, pp. $43-48$. 
Bloemer, José M. M.; De Ruyter, Ko (1998). On the Relationship between Store Image, Store Satisfaction, and Store Loyalty. European Journal of Marketing, 32, pp. 499-513.

Bloemer, José M. M.; De Ruyter, Ko; Wetzels, Martin (1999). Linking Perceived Service Quality and Service Loyalty. A Multi-Dimensional Perspective. European Journal of Marketing, 33, pp. 1082-1106.

Bolfing, Claire P.; Woodruff, Robert B. (1988). Effects of Situational Involvement on Consumers' Use of Standards in Satisfaction / Dissatisfaction Processes. Consumer Satisfaction, Dissatisfaction and Complaint Behavior, 1, pp. 16-24.

Bollen, Kenneth A.; Lennox, Richard (1991). Conventional Wisdom on Measurement. A Structural Equation Perspective. Psychological Bulletin, 110 (2), pp. 305-314.

Bolton, Ruth N. (1998). A Dynamic Model of the Duration of the Customer's Relationship with a Continuous Service Provider. The Role of Satisfaction. Marketing Science, 17 (1), pp. 45-65.

Bolton, Ruth N.; Drew, James H. (1991). A Multistage Model of Customers' Assessments of Service Quality and Value. Journal of Consumer Research, 17, pp. $375-384$.

Borrmann, Jörg; Finsinger, Jörg (1999). Markt und Regulierung. München: Vahlen.

Boulding, William; Kalra, Ajay; Staelin, Richard; Zeithaml, Valerie A. (1993). A Dynamic Process Model of Service Quality. From Expectations to Behavioral Intentions. Journal of Marketing Research, 30, pp. 7-27.

Bower, Gordon H.; Hilgard, Ernest R. (1984). Theories of Learning. Englewood Cliffs: Prentice-Hall, $3^{\text {rd }}$ edition.

Brachat, HANnes (1995). Computergestützte Kommunikationssysteme als Kundenbindungs-Netzwerk in Handel und Kundendienst. In: Reuss, Holger; Müller, WolfGANG (Hrsg.): Wettbewerbsvorteile im Automobilhandel. Frankfurt am Main/New York: Campus, S. 241-253.

Brachmann, Harald (1985). Die Absatzorganisation in der Energiewirtschaft. Frankfurt am Main: VWEW.

Brinberg, David; Wood, Ronald (1983). A Resource Exchange Theory Analysis of Consumer Behavior. Journal of Consumer Research, 10 (3), pp. 330-338.

Bruhn, MANFred (2000). Qualitätssicherung im Dienstleistungsmarketing. Eine Einführung in die theoretischen und praktischen Probleme. In: BRUHN, MANFrED; Stauss, Bernd (Hrsg.): Dienstleistungsqualität. Konzepte, Methoden, Erfahrungen. Wiesbaden: Gabler, 3. Auflage, S. 20-48.

Bruhn, Manfred (2002). Relationship Marketing. Management of Customer Relationships. Harlow, UK: Pearson.

Burnham, Thomas A.; Frels, Judy F.; Mahajan, Vijay (2003). Consumer Switching Costs. A Typology, Antecedents, and Consequences. Journal of the Academy of Marketing Science, 31 (2), pp. 109-126.

Burton, Scot; Netemeyer, Richard G. (1992). The Effect of Enduring, Situational, and Response Involvement on Preference Stability in the Context of Voting Behavior. Psychology \& Marketing, 9 (2), pp. 143-156. 
Butters, Gerard R. (1977). Equilibrium Distributions of Sales and Advertising Prices. Review of Economic Studies, 44, pp. 465-491.

CAdosch, Walter (1982). Marketingstrategien für öffentliche Energieversorgungsunternehmen. Bern/Stuttgart: Paul Haupt.

Cadotte, Ernest; Woodruff, Robert; Jenkins, Roger (1987). Expectations and Norms in Models of Consumer Satisfaction. Journal of Marketing Research, 24, pp. 305-314.

Campbell, D. T. (1963). Social Attitudes and Other Acquired Behavioral Dispositions. In: Koch, S. (Ed.): Psychology: A Study of a Science, volume 6. New York: McGrawHill, pp. 94-172.

Cattell, Raymond B.; Winder, Alvin E. (1952). Structural Rigidity in Relation to Learning Theory and Clinical Psychology. Psychological Review, 59, pp. 23-39.

Celsi, Richard L.; Olson, Jerry C. (1988). The Role of Involvement in Attention and Comprehension Processes. Journal of Consumer Research, 15 (September), pp. 210-224.

Chakravarty, Sugato; Feinberg, Richard; Rhee, Eun-Young (2004). Relationships and individuals' bank switching behavior. Journal of Economic Psychology, 25 (4), pp. 507-527.

Chaplin, William F. (1991). The Next Generation of Moderator Research in Personality Psychology. Journal of Personality, 91, pp. 143-178.

Chaplin, William F. (1997). Personality, Interactive Relations, and Applied Psychology. In: Hogan, Robert; Johnson, John; Briggs, Stephen (Eds.): Handbook of Personality Psychology. San Diego et al.: Academic Press, pp. 873-890.

Chatelin, Yves Marie; Esposito Vinzi, Vicenzo; Tenenhaus, Michel (2002). State-of-Art on PLS Path Modeling Through the Available Software. Working Paper, pp. 1-31.

Chenet, Pierre; Tynan, Caroline; Money, Arthur (1999). Service Performance Gap: Re-evaluation and Redevelopment. Journal of Business Research, 46, pp. 133147.

Chenet, Pierre; Tynan, Caroline; Money, Arthur (2000). The Service Performance Gap: Testing the Redeveloped Causal Model. European Journal of Marketing, $34(3 / 4)$, pp. 472-495.

Chin, Wynne W. (1998a). Issues and Opinion Structural Equation Modeling. MIS Quarterly, 22 (1), pp. VII-XVI.

Chin, Wynne W. (1998b). The Partial Least Squares Approach to Structural Equation Modeling. In: Marcoulides, George A. (Ed.): Modern Methods for Business Research. Mahwah, NJ: Lawrence Erlbaum Associates, Inc., pp. 295-336.

Chin, Wynne W.; Marcolin, Barbara L.; Newsted, Peter R. (2003). A Partial Least Squares Latent Variable Modeling Approach for Measuring Interaction Effects. Results from a Monte Carlo Simulation Study and an Electronic-Mail Emotion / Adopion Study. Information Systems Research, 14 (2), pp. 189-217.

Chintagunta, Pradeep K. (1998). Inertia and Variety Seeking in a Model of BrandPurchase Timing. Marketing Science, 17, pp. 253-270. 
Churchill, Gilbert A. (1979). A Paradigm for Developing Better Measures of Marketing Constructs. Journal of Marketing Research, 16, pp. 64-73.

Churchill, Gilbert A.; Suprenant, Carol (1982). An Investigation Into the Determinants of Customer Satisfaction. Journal of Marketing Research, 19, pp. 491504.

Clemens, Brigitte (1983). Strategien der Verhaltensbeeinflussung bei privaten Energienachfragern. Dissertation, Universität Berlin.

Coase, Ronald H. (1937). The Nature of the Firm. Economica, 4 (November), pp. 386-405.

Cohen, Jacob (1988). Statistical Power Analysis for the Behavioral Sciences. Hillsdale: Lawrence Erlbaum Associates, $2^{\text {nd }}$ edition.

Cohen, Jасов (1994). The Earth Is Round ( $p<.05)$. American Psychologist, 49 (12), pp. 997-1003.

Colgate, Mark; Lang, Bodo (2001). Switching Barriers in Consumer Markets: an Investigation of the Financial Services Industry. The Journal of Consumer Marketing, 18 (4), pp. 332-347.

Cornelsen, Jens (2000). Kundenwertanalysen im Beziehungsmarketing. Theoretische Grundlegung und Ergebnisse einer empirischen Studie im Automobilbereich. Dissertation, Friedrich-Alexander-Universität Erlangen-Nürnberg.

Cornelsen, Jens; Litzenroth, Heinrich A. (2002). Stromaufwärts, aber mit Gewinn. Marketing Journal, 34 (6), S. 40-43.

Cortina, Jose M. (1993). What is Coefficient Alpha. An Examination of Theory and Applications. Journal of Applied Psychology, 78 (1), pp. 98-104.

Cote, Joseph A.; McCullough, James; Reilly, Michael (1985). Effects of Unexpected Situations on Behavior-Intention Differences. Journal of Consumer Research, 12 (2), pp. 188-194.

Coupey, Eloise; Irwin, Julie; Payne, John W. (1998). Product Category Familiarity and Preference Construction. Journal of Consumer Research, 24 (4), pp. 459-467.

Cox, Donald F. (1967a). Risk Handling in Consumer Behavior. An Intensive Study of Two Cases. In: Cox, Donald F. (Ed.): Risk Taking and Information Handling in Consumer Behavior. Cambridge, MA: Harvard University Press, pp. 34-81.

Cox, Donald F. (Ed.): (1967b). Risk Taking and Information Handling in Consumer Behavior. Cambridge, MA: Harvard University Press.

Cronbach, Lee J. (1951). Coefficient Alpha and the Internal Structure of Tests. Psychometrika, 16 (3), pp. 297-334.

Cronin, Joseph J.; Taylor, Steven A. (1992). Measuring Service Quality. A Reexamination and Extension. Journal of Marketing, 56, pp. 55-68.

Crosby, Lawrence A.; Evans, Kenneth R.; Cowles, Deborah (1990). Relationship Quality in Services Selling. An Interpersonal Influence Perspective. Journal of Marketing, 54, pp. 68-81.

Crosby, Lawrence A.; Stephens, Nancy (1987). Effects of Relationship Marketing on Satisfaction, Retention, and Prices in the Life Insurance Industry. Journal of Marketing Research, 24, pp. 404-411.

Crosby, Lawrence A.; Taylor, James R. (1983). Psychological Commitment and 
Its Effects on Post-Decision Evaluation and Preference Stability Among Voters. Journal of Consumer Research, 9, pp. 413-431.

Czepiel, John A.; Gilmore, Robert (1987). Exploring the Concept of Loyalty in Services. In: Czepiel, J. A.; Congram, C. A.; Shanahan, J. (Eds.): The Services Challenge. Integrating for Competitive Advantage. Chicago, IL: American Marketing Association, pp. 91-94.

Datamonitor (1999). Customer Management in European Utilities. Developing Effective Customer Acqusition and Retention Strategies.

Day, George S. (2000). Managing Market Relationships. Journal of the Academy of Marketing Science, 28 (1), pp. 24-30.

Day, Ralph L. (1977). Extending the Conept of Consumer Satisfaction. Advances in Consumer Research, 4, pp. 149-154.

De Ruyter, Ko; Bloemer, José M. M.; Peeters, Pascal (1997). Merging Service quality and service satisfaction. And empirical test of an integration model. Journal of Economic Psychology, 18, pp. 387-406.

De Wulf, Kristof; Odekerken-Schröder, Gaby; Iacobucci, Dawn (2001). Investments in Consumer Relationship. A Cross-Country and Cross-Industry Exploration. Journal of Marketing, 65 (October), pp. 33-50.

Diamantopoulos, Adamantios; Winklhofer, Heidi M. (2001). Index Construction with Formative Indicators: An Alternative to Scale Development. Journal of Marketing Research, 38 (May), pp. 269-277.

Diamond, Peter A. (1971). A Model of Price Adjustment. Journal of Economic Theory, 3, pp. 156-168.

Dick, Alan S.; Basu, Kunal (1994). Customer Loyalty Toward an Integrated Conceptual Framework. Journal of the Academy of Marketing Science, 22, pp. 99-113.

Diller, Hermann (1996). Kundenbindung Als Marketingziel. Marketing · Zeitschrift für Forschung und Praxis, 18 (2), S. 81-94.

Doney, Patricia M.; Cannon, Joseph P. (1997). An Examination of the Nature of Trust in Buyer-Seller Relationships. Journal of Marketing, 61, pp. 35-51.

Dowling, Grahame R.; Staelin, Richard (1994). A Model of Perceived Risk and Intended Risk-Handling Activity. Journal of Consumer Research, 21 (June), pp. 119134.

Duhan, D.; Johnson, S.; Wilcox, J.; Harrell, G. (1997). Influences on Consumer Use of Word-of-Mouth Recommendation Sources. Journal of the Academy of Marketing Science, 25 (4), pp. 283-295.

Dwyer, F. Robert; Schurr, Paul H.; Oh, Sejo (1987). Developing Buyer-SellerRelationships. Journal of Marketing, 51 (April), pp. 11-27.

Eagly, Alice H.; Chaiken, S. (1993). The Impact of Attitudes on Behaviors. In: The Psychology of Attitudes. Fort Worth, TX: Harcourt Brace Jovanovich, chapter 4, pp. 155-218.

Efron, Bradley; Tibshirani, Robert J. (1993). An Introduction to the Bootstrap. New York: Chapman \& Hall.

EgGert, Andreas (1999). Kundenbindung aus Kundensicht. Konzeptualisierung, Operationalisierung, Verhaltenswirksamkeit. Wiesbaden: Gabler. 
Eggert, Andreas (2003). Die zwei Perspektiven des Kundenwerts. Darstellung und Versuch einer Integration. In: Günter, Bernd; Helm, SABrina (Hrsg.): Kundenwert. Grundlagen, Innovative Konzepte, Praktische Umsetzungen. Wiesbaden: Gabler, S. $41-59$.

Eggert, Andreas; FAssott, Georg (2003). Zur Verwendung formativer und reflektiver Indikatoren in Strukturgleichungsmodellen. Ergebnisse einer Meta-Analyse und Anwendungsempfehlungen. Kaiserslauterer Schriftenreihe Marketing, 20.

Eggert, Andreas; Helm, Sabrina (2000). Determinanten der Weiterempfehlung. Kundenzufriedenheit oder Kundenbindung? Der Markt, 39 (2), S. 63-72.

EickHof, Norbert (1998). Wettbewerb nun auch bei der Energieversorgung. Wirtschaftswissenschaftliches Studium, 27 (9), S. 437.

Emerson, Richard M. (1976). Social Exchange Theory. Annual Review of Sociology, 2, pp. 350-362.

Engel, James F.; Blackwell, Roger D.; Miniard, Paul W. (1986). Consumer Behavior. Dryden, $5^{\text {th }}$ edition.

Europäisches Parlament und Rat der Europäischen Union (1996). EURichtlinie Elektrizität. Richtlinie 96/92/EG des Europäischen Parlaments und des Rates vom 19. Dezember 1996 betreffend gemeinsame Vorschriften für den Elektrizitätsbinnenmarkt. Amtsblatt der Europäischen Gemeinschaften Nr. L 27/20 DE vom 30.01.97.

Farrell, Daniel; Rusbult, Caryl E. (1981). Exchange Variables as Predictors of Job Satisfaction, Job Commitment, and Turnover. The Impact of Rewards, Costs, Alternatives, and Investments. Organizational Behavior and Human Performance, 28, pp. 78-95.

Farrell, Joseph; Shapiro, Carl (1988). Dynamic Competition With Switching Costs. The RAND Journal of Economics, 19 (1), pp. 123-137.

Festinger, Leon (1957). A Theory of Cognitive Dissonance. Stanford: Stanford University Press.

Fishbein, Martin (1980). A Theory of Reasoned Action. Some Applications and Implications. In: Howe, JR., Herbert E.; PAGe, Monte M. (Eds.): Beliefs, Attitudes, and Values. Nebraska Symposium on Motivation 1979, 27. Lincoln/London: University of Nebraska Press, pp. 65-116.

Fishbein, Martin; Ajzen, ICEk (1975). Belief, Attitude, Intention and Behavior. An Introduction to Theory and Research. Reading, MA: Addison-Wesley.

Fornell, Claes (Ed.): (1982). A Second Generation of Multivariate Analysis. Methods, Volume I. New York: Praeger.

Fornell, Claes (1992). A National Customer Satisfaction Barometer. The Swedish Experience. Journal of Marketing, 56, pp. 6-21.

Fornell, Claes (1995). The Quality of Economic Output. Empirical Generalizations About its Distribution and Relationship to Market Share. Marketing Science, 14 (3), pp. G203-G211.

Fornell, Claes; Larcker, David F. (1981). Evaluating Structural Equation Models with Unobservable Variables and Measurement Error. Journal of Marketing Research, 18, pp. 39-50.

Fornell, Claes; Wernerfelt, Birger (1987). Defensive Marketing Strategy by 
Customer Complaint Management: A Theoretical Analysis. Journal of Marketing Research, 24, pp. 337-346.

Fournier, Susan (1998). Consumers and their Brands: Developing Relationship Theory in Consumer Research. Journal of Consumer Research, 24 (March), pp. 343-373.

Fournier, Susan; Mick, David Glen (1999). Rediscovering Satisfaction. Journal of Marketing, 63 (October), pp. 5-23.

Freyland, Bettina; Herrmann, Andreas; Huber, Frank (1999). Warum sind zufriedene Kunden nicht treu? Versicherungswirtschaft, 54 (23), S. 1744-1747.

Fritz, Wolfgang; König, Siegfried (2000). Der liberalisierte Strommarkt. Eine Einführung. In: Kahmann, Martin; KÖNig, Siegfried (Hrsg.): Wettbewerb im liberalisierten Strommarkt. Regeln und Techniken. Berlin/Heidelberg: Springer, S. 325.

Fudenberg, Drew; Tirole, Jean (1984). The Fat-Cat Effect, the Puppy-Dog Ploy, and the Lean and Hungry Look. American Economic Review, 74, pp. 361-366.

Fusser, Andreas (2002). Was macht wild auf den Wechsel. Es braucht Extra-Anreize um Neukunden für Stromanbieter zu gewinnen. In: Angres, Volker; RIEFleR, SteFAN (Hrsg.): Wechsellust und Wechselfrust. Was bewegt Verbraucher zu nachhaltigem Konsum, S. 7-8. Grenzach-Wyhlen: NaturEnergie.

Ganesan, Shankar (1994). Determinants of Long-Term Orientation in Buyer-Seller Relationships. Journal of Marketing, 58, pp. 1-19.

Ganesh, Jaishankar; Arnold, Mark J.; Reynolds, Kristy Ellis (2000). Understanding the Customer Base of Service Providers. An Examination of the Differences between Switchers and Stayers. Journal of Marketing, 64 (3), pp. 65-87.

Garland, Barbara C.; Westbrook, Robert A. (1989). An Exploration of Client Satisfation in a Nonprofit Context. Journal of the Academy of Marketing Science, 17, pp. 297-303.

Gemünden, Hans Georg (1985). Perceived Risk and Information Search. A Systematic Meta-Analysis of the Empirical Evidence. International Journal of Research in Marketing, 2 (2), pp. 79-100.

Gerbing, David W.; Anderson, James C. (1988). An Updated Paradigm for Scale Development Incorporating Unidimensionality and its Assessment. Journal of Marketing Research, 25, pp. 186-192.

Gerpott, Torsten J.; Rams, Wolfgang (2000). Kundenbindung, -loyalität und zufriedenheit im deutschen Mobilfunkmarkt. Die Betriebswirtschaft, 60 (6), S. 738-755.

Gerrard, Philip; Cunningham, Barton J. (2004). Consumer switching behavior in the Asian banking market. Journal of Services Marketing, 18 (3), pp. 215-223.

Giering, Annette (2000). Der Zusammenhang zwischen Kundenzufriedenheit und Kundenloyalität. Eine Untersuchung moderierender Effekte. Wiesbaden: Gabler.

GierL, Heribert (1993). Zufriedene Kunden als Markenwechsler. Absatzwirtschaft, 36 (2), S. 90-94.

Glynn, Leisa Reinecke; Goldsmith, Ronald E. (1993). Application of the Personal Involvement Inventory in Marketing. Psychology \& Marketing, 10 (4), pp. 357-366.

Goodman, Paul S.; Fichman, Mark; Lerch, F. Javier; Snyder, Pamela R. 
(1995). Customer-Firm Relationships, Involvement, and Customer Satisfaction. Academy of Management Journal, 38 (5), pp. 1310-1324.

Gotlieb, Jerry B.; Grewal, Dhruv; Brown, Stephen W. (1994). Consumer Satisfaction and Perceived Quality: Complementary or Divergent Constructs? Journal of Applied Psychology, 79 (6), pp. 875-885.

Gotlieb, Jerry B.; Schlacter, John L.; St. Louis, Robert D. (1992). Consumer Decision Making: A Model of the Effects of Involvement, Source Credibility, and Location on the Size of the Price Difference Required to Induce Consumers to Change Suppliers. Psychology \& Marketing, 9 (3), pp. 191-208.

Götz, Oliver; Lienr-Gobbers, Kerstin (2004). Der Partial-Least-Squares (PLS)Ansatz zur Analyse von Strukturgleichungsmodellen. Arbeitspapier 2, Institut für Marketing.

Graehl, Sven; Dreher, Martin; Wietschel, Martin; Rentz, Otto (2001). Eine Analyse des Marktes für Grüne Angebote in Deutschland. Zeitschrift für Energiewirtschaft, 25 (4), S. 221-230.

Greenwald, Anthony G.; Leavitt, Clark (1984). Audience Involvement in Advertising. Four levels. Journal of Consumer Research, 11, pp. 581-592.

Gronhaug, KJell (1977). Exploring Consumer Complaining Behavior. A Model and Some Empirical Results. In: PERRAult, W. D. (Ed.): Advances in consumer research. Provo, UT: Association for Consumer Research, pp. 159-165.

Guiltinan, Joseph P. (1989). A Classification of Switching Costs With Implications for Relationship Marketing. In: Childers, Terry L.; Bagozzi, Richard P.; Peter, J. PAul (Eds.): 1989 AMA Winter Educators' Conference. Marketing Theory and Praxis, pp. 216-220. Chicago: American Marketing Association.

Gwinner, Kevin P.; Gremler, Dwayne D.; Bitner, Mary Jo (1998). Relational Benefits in Service Industries. The Customer's Perspective. Journal of the Academy of Marketing Science, 26, pp. 101-114.

HaAs, Lothar (1975). Marketingstrategien in der Elektrizitätswirtschaft. Dissertation, Universität zu Köln, München.

Hallén, Lars; Johanson, Jan; Seyed-Mohamed, Nazeem (1991). Interfirm Adaptation in Business Relationships. Journal of Marketing, 55 (2), pp. 29-37.

Hallowell, Roger (1996). The Relationships of Customer Satisfaction, Customer Loyalty, and Profitability. An Empirical Study. International Journal of Service Industry Management, 7 (4), pp. 27-42.

Harman, Harry H. (1976). Modern Factor Analysis. Chicago et al.: University of Chicago Press.

Hauser, John R.; Simester, Duncan I.; Wernerfelt, Birger (1994). Customer Satisfaction Incentives. Marketing Science, 13, pp. 327-350.

Hauser, John R.; Wernerfelt, Birger (1990). An Evaluation Cost Model of Consideration Sets. Journal of Consumer Research, 16, pp. 393-408.

Hausknecht, Douglas R. (1990). Measurement Scales in Consumer Satisfaction/ Dissatisfaction. Journal of Consumer Satisfaction, Dissatisfaction and Complaining Behavior, 3, pp. 1-11. 
Heeler, Roger M.; Ray, Michael L. (1972). Measure Validation in Marketing. Journal of Marketing Research, 9, pp. 361-370.

Heger, GüNTHER (2003). Aspekte der Kundenbindung bei Energieversorgungsunternehmen. In: Rese, Mario; Söllner, Albrecht; Utzig, B. Peter (Hrsg.): Relationship Marketing. Standortbestimmung und Perspektiven. Berlin/Heidelberg: Springer, S. 241-271.

Helm, Sabrina; Eggert, Andreas; Fassott, Georg (2005). Identifikation und Quantifizierung moderierender und mediierender Effekte in komplexen Kausalstrukturen. In: Bliemel, Friedhelm W.; Eggert, Andreas; Fassott, Georg; HenseLER, Jörg (Hrsg.): Handbuch PLS-Pfadmodellierung. Methode, Anwendung, Praxisbeispiele. Stuttgart: Schaeffer-Poeschel. Im Druck.

Hennig-Thurau, Thorsten; Gwinner, Kevin P.; Gremler, Dwayne D. (2002). Understanding Relationship Marketing Outcomes. An Integration of Relational Benefits and Relationship Quality. Journal of Service Research, 5 (February), pp. 230-247.

Henseler, Jörg; Hoffmann, Thorsten (2003). Kundenwert als Baustein zum Unternehmenswert. Hamburg: Dr. Kovač.

Hentschel, Bert (1991). Beziehungsmarketing. Das Wirtschaftsstudium, 20 (1), S. $25-28$.

Hentschel, Bert (2000). Multiattributive Messung von Dienstleistungsqualität. In: Bruhn, Manfred; Stauss, Bernd (Hrsg.): Dienstleistungsqualität. Konzepte, Methoden, Erfahrungen. Wiesbaden: Gabler, 3. Auflage, S. 289-320.

Herrmann, Andreas (1998). Produktmanagement. München: Vahlen.

Herrmann, Andreas; Huber, Frank; Braunstein, Christine (2000). Ein Erklärungsansatz der Kundenbindung unter Berücksichtigung der wahrgenommenen Handlungskontrolle. Die Betriebswirtschaft, 3 (60), S. 293-313.

Heskett, James L.; Jones, Thomas O.; Loveman, Gary W. et al. (1994). Putting the service-profit chain to work. Harvard Business Review, 72, pp. 164-174.

Heskett, James L.; Sasser, Jr., W. Earl; Hart, Christopher W.L. (1991). Service breakthroughs. Changing the Rules of the Game. New York: Free Press et al.

Heslin, Richard; Johnson, Blair T. (1992). Prior Involvement and Incentives to Pay Attention to Information. Psychology \& Marketing, 9 (3), pp. 209-219.

Hinde, Robert A. (1979). Towards Understanding Relationships. London et al.: Academic Press Inc.

Hirshleifer, David; Welch, Ivo (2001). An Economic Approach to the Psychology of Change. Amnesia, Inertia, and Impulsiveness. Cowles Foundation Discussion Paper, 1306 .

Hom, Peter W.; Kinicki, Angelo J. (2001). Toward a Greater Understanding of how Satisfaction Drives Employee Turnover. Academy of Management Journal, 44 (5), pp. $975-987$.

Homans, George Caspar (1958). Social Behavior as Exchange. American Journal of Sociology, 63, pp. 597-606.

Homans, George Caspar (1961). Social Behavior: Its Elementary Forms. New York et al.: Harcourt Brace Jovanovich. 
Homans, George Caspar (1974). Social Behavior: Its Elementary Forms. New York et al.: Harcourt Brace Jovanovich, $2^{\text {nd }}$ edition.

Homburg, Christian (1992). Die Kausalanalyse. Eine Einführung. Wirtschaftswissenschaftliches Studium, 21 (10), S. 499-508.

Homburg, Christian; Baumgartner, Hans (1998). Beurteilung von Kausalmodellen. Bestandsaufnahme und Anwendungsempfehlungen. In: HildeBrandt, Lutz; HomBURG, Christian (Hrsg.): Die Kausalanalyse. Instrument der empirischen betriebswirtschaftlichen Forschung. Stuttgart: Schäffer-Poeschel, S. 343-369.

Homburg, Christian; Giering, Annette (1996). Konzeptualisierung und Operationalisierung komplexer Konstrukte. Ein Leitfaden für die Marketingforschung. Marketing - Zeitschrift für Forschung und Praxis, 18 (1), S. 5-24.

Homburg, Christian; Giering, Annette; Hentschel, Frederike (1999). Der Zusammenhang zwischen Kundenzufriedenheit und Kundenbindung. Die Betriebswirtschaft, 59 (2), S. 173-195.

Homburg, Christian; Rudolph, Bettina (1998). Die Kausalanalyse als Instrument zur Messung der Kundenzufriedenheit im Industriegütermarketing. In: HILDEBRANDT, Lutz; Homburg, Christian (Hrsg.): Die Kausalanalyse. Instrument der empirischen betriebswirtschaftlichen Forschung. Stuttgart: Schäffer-Poeschel, S. 237-264.

Homburg, Christian; Schnurr, Phoebe (1998). Kundenwert als Instrument der Wertorientierten Unternehmensführung. In: BRUhn, MANFRED; Lusti, Markus; MülleR, WERneR R. (Hrsg.): Wertorientierte Unternehmensführung. Perspektiven und Handlungsfelder für die Wertsteigerung von Unternehmen. Festschrift zum 10jährigen Bestehen des Wirtschaftswissenschaftlichen Zentrums (WWZ) der Universität Basel. Wiesbaden: Gabler, S. 169-189.

Homburg, Christian; Werner, Harald (1998). Messung und Management von Kundenzufriedenheit. Marktforschung und Management, 42 (4), S. 131-135.

Höser, H. (1998). Kontextabhängige Präferenzen. Frankfurt am Main: Peter Lang.

Houston, Franklin S.; Gassenheimer, Jule B. (1987). Marketing and Exchange. Journal of Marketing, 51 (4), pp. 3-18.

Houston, Michael J.; Rothschild, Michael L. (1978). Conceptual and Methodological Perspectives on Involvement. In: JAIN, S. C. (Ed.): Educator's proceedings. Chicago: American Marketing Association, pp. 184-187.

Hoyer, W. D.; Ridgway, N. M. (1984). Variety seeking as an Explanation for Exploratory Purchase Behavior. A Theoretical Model. Journal of Consumer Research, 11, pp. 114-119.

Hoyer, Wayne D.; MacInnis, Deborah J. (1997). Consumer Behavior. Boston, MA: Houghton Miffin.

Huang, Ming-Hui; Yu, Shinti (1999). Are Consumers Inherently or Situationally Brand Loyal? A Set Intercorrelation Account for Conscious Brand Loyalty and Nonconcious Inertia. Psychology \& Marketing, 16 (6), pp. 523-544.

Huff, James O.; Huff, Anne S.; Thomas, Howard (1992). Strategic Renewal and the Interaction of Cumulative Stress and Inertia. Strategic Management Journal, 13, pp. $55-75$.

Hulland, John (1999). Use of Partial Least Squares (PLS) in Strategic Management 
Research. A Review of Four Recent Studies. Strategic Management Journal, 20, pp. 195-204.

Iacobucci, Dawn; Ostrom, Andy (1996). Commercial and Interpersonal Relationships. Using the Structure of Interpersonal Relationships to Understand Individual-toindividual, Individual-to-firm and Firm-to-firm Relationships in Commerce. International Journal of Research in Marketing, 13, pp. 53-72.

Iverson, Roderick D.; Roy, Parimal (1994). A Causal Model of Behavioral Commitment. Evidence From a Study of Australian Blue-collar Employees. Journal of Management, 20 (1), pp. 15-41.

JaCKSOn, BARBARA B. (1985). Winning and Keeping Industrial Customers. The Dynamics of Customer Relationships. Lexington, MA: Lexington Books.

Jacoby, Jacob W.; Kaplan, Leon B. (1972). The Components of Perceived Risk, Volume III. working paper, Chicago, Association for Consumer Research.

Jarvis, Cheryl B.; Mackenzie, Scott B.; Podsakoff, Philip M. (2003). A Critical Review of Construct Indicators and Measurement Model Misspecification in Marketing and Consumer Research. Journal of Consumer Research, 30 (2), pp. 199218.

Jen, William; Hu, Kai-Chien (2003). Application of Perceived Value Model to Identify Factors Affecting Passengers' Repurchase Intentions on City Bus. A Case of the Taipei Metropolitan Area. Journal of Consumer Research, 30, pp. 307-327.

Johnson, Michael P. (1982). Social and Cognitive Features of the Dissolution of Commitment to Relationships. In: Duck, Steve (Ed.): Personal Relationships. 4. Dissolving Personal Relationships. London/New York: Academic Press, chapter 3, pp. $51-73$.

Jones, Michael A.; Mothersbaugh, David L.; Beatty, Sharon E. (2000). Switching Barriers and Repurchase Intentions in Services. Journal of Retailing, 76 (2), pp. 259-274.

Jones, Michael A.; Mothersbaugh, David L.; Beatty, Sharon E. (2002). Why Customers Stay. Measuring the Underlying Dimensions of Services Switching Costs and Managing their Differential Service Outcomes. Journal of Business Research, 55 (6), pp. 441-450.

Jones, Thomas O.; Sasser, Jr., W. Earl (1995). Why Satisfied Customers Defect. Harvard Business Review, 73 (6), pp. 88-99.

Jöreskog, Karl G.; Sörbom, Dag (1979). Advances in Factor Analysis and Structural Equation Models. Cambridge: Abt. Ass.

Jöreskog, Karl G.; Wold, Herman (Eds.): (1982). Systems under Indirect Observation. Causality, Structure, Prediction. Part I. Contributions to Economic Analysis. Amsterdam/New York/Oxford: North-Holland.

Julander, Claes-Robert; Söderlund, Magnus (2003). Effects of Switching Barriers on Satisfaction, Repurchase Intentions and Attitudinal Loyalty. SSE/EFI Working Paper Series in Business Administration, 1.

Kaldor, Nicholas (1935). Market Imperfection and Excess Capacity. Economica, 2.

KAnther, Verena (2001). Facetten hybriden Kaufverhaltens. Ein kausalanalytischer 
Erklärungsansatz auf Basis des Involvement-Konstrukts. Wiesbaden: Deutscher Universitäts-Verlag.

Kapferer, Jean-Noel; Laurent, Gilles (1993). Further Evidence on the Consumer Involvement Profile: Five Antecedents of Involvement. Psychology \& Marketing, 10 (4), pp. 347-355.

Karakaya, Fahri; Stahl, Michael J. (1989). Barriers to Entry and Market Decisions in Consumer and Industrial Good Markets. Journal of Marketing, 53, pp. 80-91.

Keaveney, Susan M. (1995). Customer Switching Behavior in Service Industries. An Exploratory Study. Journal of Marketing, 59 (2), pp. 71-82.

Keller, Bernhard; Matzke, SAndro (2000). Marketing-Instrumente zur Gewinnung und Bindung von Kunden. In: Kahmann, MARTin; König, Siegfried (Hrsg.): Wettbewerb im liberalisierten Strommarkt. Regeln und Techniken. Berlin/Heidelberg: Springer, S. 41-64.

Kelley, Harold H.; Thibaut, John W. (1978). Interpersonal Relationships. A Theory of Interdependence. New York: Wiley.

Kerin, Roger A.; Varadarajan, P. Rajan; Peterson, Robert A. (1992). FirstMover Advantage. A Synthesis, Conceptual Framework, and Research Propositions. Journal of Marketing, 56, pp. 33-52.

Kim, Moon-Koo; Park, Myeong-Cheol; Jeong, Dong-Heon (2004). The Effects of Customer Satisfaction and Switching Barrier on Customer Loyalty in Korean Mobile Telecommunication Services. Telecommunications Policy, 28, pp. 145-159.

Kirmani, Amna; Rao, Akshay R. (2000). No Pain, No Gain: A Critical Review of the Literature on Signaling Unobservable Product Quality. Journal of Marketing, 64 (2), pp. 66-79.

Klemperer, Paul D. (1987a). The Competitiveness of Markets with Switching Costs. The RAND Journal of Economics, 18 (1), pp. 138-150.

Klemperer, Paul D. (1987b). Markets With Consumer Switching Costs. The Quarterly Journal of Economics, 102, pp. 375-394.

Klemperer, Paul D. (1995). Competition When Consumers Have Switching Costs. An Overview With Applications to Industrial Organization, Macroeconomics and International Trade. Review of Economic Studies, 62, pp. 515-539.

Klopfer, Thomas; Schulz, Walter (1993). Märkte für Strom. Internationale Erfahrungen und Übertragbarkeit auf Deutschland. München: Oldenburg.

Kloubert, Thomas (2001). Analyse und Beeinflussung des Wechselverhaltens privater Nachfrager im Energiemarkt. Theoretische und konzeotionelle Ansätze aus der Perspektive neuer Anbieter. München: Kloubert.

Knapp, M. L. (1984). Interpersonal Communication and Human Relationships. Boston, MA: Allyn \& Bacon.

Knoester, Chris; Booth, Alan (2000). Barriers to Divorce. When Are They Effective? When Are They Not? Journal of Family Issues, 21 (1), pp. 78-99.

Kommission Der EuropäIschen Union (2001). Commission Staff Working Paper. First report on the implementation of the internal electricity and gas market. Arbeitspapier, Kommission der Europäischen Union.

Koppelmann, Udo (1969). Zum Erkenntnisobjekt Ware. In: Koppelmann, Udo 
(Hrsg.): Die Ware in Wirtschaft und Technik. Festschrift zum 65. Geburtstag von Artur Kutzelnigg. Berlin: Herne, S. 15-20.

Koppelmann, Udo (1997). Produktmarketing. Entscheidungsgrundlagen für Produktmanager, Band 5. vollständig überarb. und erw. Aufl. Berlin et al.: Springer.

Kotler, Philip; Bliemel, Friedhelm W. (2001). Marketing Management. Analyse, Planung und Verwirklichung. Stuttgart: Schäffer-Poeschel, 10. Auflage.

Kranton, Rachel E. (1996). The Formation of Cooperative Relationships. Journal of Law, Economics, and Organization, 12, pp. 214-233.

Krech, David et AL. (1992). Grundlagen der Psychologie. Weinheim: Beltz.

Krengel, UlRich (1991). Einführung in die Wahrscheinlichkeitstheorie und Statistik. Braunschweig: Vieweg, 3. Auflage.

Krick, Matthias (1999). Kundenbeziehungen werden zu Produkten. Absatzwirtschaft, 42 (8), S. 58-63.

Kroeber-RIEL, Werner (1991). Strategie und Technik der Werbung. Verhaltenswissenschaftliche Ansätze. Stuttgart: Kohlhammer, 3. Auflage.

Kroeber-Riel, Werner; Weinberg, Peter (2003). Konsumentenverhalten. München: Vahlen, 8. Auflage.

Krugman, Herbert E. (1965). The Impact of Television Advertising: Learning without Involvement. The Public Opinion Quarterly, 29 (3), pp. 349-356.

Krugman, Herbert E. (1966). The impact of advertising involvement. Public Opinion Quarterly, 30, pp. 583-596.

Kruse, Joachim (2000). Kommentar zu Hans-Georg W. Voß' „Methodologische Probleme der Familienentwicklungsforschung “. In: SchneEwind, Klaus A. (Hrsg.): Familienpsychologie im Aufwind. Brückenschläge zwischen Forschung und Praxis. Göttingen: Hogrefe, S. 117-126.

Kuss, Alfred; Tomczak, Torsten (2000). Käuferverhalten. Stuttgart: Lucius \& Lucius, 2. Auflage.

Laker, Michael; Herr, Stefan (2000). Zehn Thesen zur Maktentwicklung. In: LAKeR, MichaEL (Hrsg.): Marketing für Energieversoger. Kunden binden und gewinnen im Wettbewerb. Wien/Frankfurt: Ueberreuter, S. 17-28.

Laker, Michael; Pohl, Alexander; Dahlhoff, Denise (1998). Chance durch Kundenbindung. Absatzwirtschaft, 41 (Jg. 6), S. 50-56.

Laurent, Gilles; Kapferer, Jean-Noel (1985). Measuring consumer involvement profiles. Journal of Marketing Research, 22, pp. 41-53.

Lee, Jonathan; Lee, Janghyuk; Feick, LaWrence (2001). The Impact of Switching Costs on the Customer Satisfaction-Loyalty Link. Mobile Phone Service in France. Journal of Services Marketing, 15, pp. 35-48.

LEUnG, AdA (2001). Understanding Innovation Diffusion with Social Exchange Theory. In: Winter Marketing Educators' Conference. Conference Proceedings, pp. 314-324. American Marketing Association.

Liska, Allen E. (1984). A Critical Examination of the Causal Structure of the Fishbein/Ajzen Attitude-Behavior Model. Social Psychology Quarterly, 47, pp. 61-74.

Littlejohn, Stephen W. (1996). Theories of Human Communication. Belmont, CA: Wadsworth, $5^{\text {th }}$ edition. 
LÖBBe, SABIne (1993). Marktdynamik und Dienstleistungsstrategien für EVU. Dissertation, Universität Saarbrücken, Saarbrücken.

Lohmann, Florian (1997). Loyalität Von Bankkunden. Bestimmungsgrößen und Gestaltungsmöglichkeiten. Wiesbaden: Gabler.

Lohmöller, Jan Bernd (1989). Latent Variable Path Modeling with Partial Least Squares. Heidelberg: Physica.

Loudon, David L.; Della Bitta, Albert J. (1993). Consumer Behavior. New York et al.: McGraw-Hill, $4^{\text {th }}$ edition.

Lubin, Paul C. (1992). Keeping the bank Customer Satisfied. Bankers Monthly, 109 (6), pp. 25-26.

Ludwig, Marc Alexandre (2000). Beziehungsmanagement im Internet. Eine Analyse der Informationsbedürfnisse auf Konsumgütermärkten und der Möglichkeiten ihrer Befriedigung durch Beziehungsmanagement unter Nutzung des Internets. Lohmar/Köln: Eul.

Madden, Thomas J.; Scholder Ellen, Pamela; Ajzen, Icek (1992). A Comparison of the Theory of Planned Behavior and the Theory of Reasoned Action. Personality and Social Psychology Bulletin, 18 (1), pp. 3-9.

MAhleRt, KARL (1967). Marketing in der Energie- und Wasserversorgung. München: Oldenbourg.

Mann, Ernst Werner; Laker, Michael (1997). Kundenorientierung eines Elektrizitätsversorgungsunternehmens. In: Simon, Hermann; Homburg, Christian (Hrsg.): Kundenzufriedenheit. Konzepte, Methoden, Erfahrungen. Wiesbaden: Gabler, 2. Auflage, S. 461-489.

Mano, Haim (1997). Affect and Persuasion: The Influence of Pleasentness and Arousal on Attitude Formation and Message Elaboration. Psychology \& Marketing, 14 (4), pp. 315-335.

Manski, Charles F. (1990). The Use of Intentions Data to Predict Behavior: A Best Case Analysis. Journal of the American Statistical Association, 85, pp. 934-940.

Mathur, Anil (1996). Older Adults' Motivations for Gift Giving to Charitable Organizations: An Exchange Theory Perpective. Psychology \& Marketing, 13 (1), pp. 107-123.

Maute, Manfred M.; Forrester, William R. (1993). The Structure of Consumer Complaint Intentions and Behavior. Journal of Economic Psychology, 14, pp. 219-247.

Mazursky, David; LaBarbera, Priscilla; Aiello, Al (1987). When Consumers Switch Brands. Psychology \& Marketing, 4, pp. 17-30.

McAlister, Leigh; Pessemier, Edgar (1982). Variety-seeking Behavior. An Interdisciplinary Review. Journal of Consumer Research, 9, pp. 311-322.

McCall, John J. (1965). The Economics of Information and Optimal Stopping Rules. Journal of Business, 38, pp. 300-317.

McQuarrie, Edward F.; Munson, J. Michael (1987). The Zaichkowsky Involvement Inventory: Modification and Extension. In: Wallendorf, Mellanie; AnderSEn, PAul (Eds.): Advances in Consumer Research, volume 14. Provo: Association for Consumer Research, pp. 36-40.

Meyer, Anton; Dornach, Frank (2000). Kundenmonitor Deutschland. Qualität und 
Kundenorientierung. Jahrbuch der Kundenorientierung in Deutschland 2000. München: Servicebarometer AG.

Miniard, Paul W.; Cohen, Joel B. (1979). Isolating Attitudinal and Normative Influences in Behavioral Intentions Models. Journal of Marketing Research, 16 (1), pp. $102-110$.

Mittal, BANWARI (1982). Understanding the Bases and Effects of Involvement in the Consumer Choice Process. PhD Thesis, University of Pittsburgh.

Mittal, Banwari (1995). A Comparative Analysis of Four Scales of Consumer Involvement. Psychology \& Marketing, 12 (7), pp. 663-682.

Mittal, Banwari; Lee, Myung S. (1988). Separating brand choice involvement from producer involvement via consumer involvement profiles. Advances in Consumer Research, 15, pp. 43-49.

Mittal, Vikas; Ross, William T.; Baldasare, Patrick M. (1998). The Asymmetric Impact of Negative and Positive Attribute-Level Performance on Overall Satisfaction and Repurchase Intentions. Journal of Marketing, 62, pp. 33-47.

Molm, Linda D. (1994). Dependence and Risk: Transforming the Structure of Social Exchange. Social Psychology Quarterly, 57 (3), pp. 163-176.

Monroe, Kent B.; Dodds, William B. (1988). A Research Program for Establishing the Validity of the Price-Quality Relationship. Journal of the Academy of Marketing Science, 16 (1), pp. 151-168.

Morgan, Robert M.; Hunt, Shelby D. (1994). The Commitment-Trust Theory of Relationship Marketing. Journal of Marketing, 58, pp. 20-38.

Müller, WolfGang; RIEsEnBeck, Hans-JoAChim (1991). Wie aus zufriedenen auch anhängliche Kunden werden. Harvard Manager, 13 (3), S. 67-79.

Muncy, James A.; Hunt, Shelby D. (1984). Consumer involvement. Definitional issues and research directions. In: KInNEAR, T.C. (Ed.): Advances in Consumer Research, volume 11. Ann Arbor, MI: Association for Consumer Research, pp. 193195.

Neal, William D. (1999). Satisfaction is Nice, but Value Drives Loyalty. Marketing Research, 11 (1), pp. 21-23.

Nielson, Charles C. (1996). An Empirical Examination of Switching Cost Investments in Business-to-Business Marketing Relationships. Journal of Business and Industrial Marketing, 11 (6), pp. 38-60.

Nord, Walter R.; Peter, J. Paul (1980). A Behavior Modification Perspective on Marketing. Journal of Marketing, 44, pp. 36-47.

Nunnally, Jum C. (1978). Psychometric Theory. New York et al.: McGraw-Hill, $2^{\text {nd }}$ edition.

Oevermann, Dirk (1996). Kundenbindungsmanagement von Kreditinstituten. Dissertation, Universität München, München.

OFGEM (2002). Electricity Supply Competition. An OFGEM Occasional Paper. working paper 83/02, The Office of Gas and Electricity Markets.

Oliva, Terence A.; Oliver, Richard L.; MacMillan, Ian C. (1992). A Catastrophe Model for Service Satisfaction Strategies. Journal of Marketing, 56, pp. 83-95.

Oliver, Richard; Swan, John E. (1989). Consumer Perceptions of Interpersonal Eq- 
uity and Satisfaction in Transactions. A Field Survey Approach. Journal of Marketing, 53, pp. 21-35.

Oliver, Richard L. (1980). A Cognitive Model of the Antecedents and Consequences of Satisfaction Decisions. Journal of Marketing Research, 17 (4), pp. 460-469.

Oliver, Richard L. (1981). Measurement and Evaluation of Satisfaction Processes in Retail Settings. Journal of Retailing, 57, pp. 25-48.

Oliver, Richard L. (1993). A conceptual model of sevice quality and service satisfaction. Compatible goals, different concepts. In: SwARTZ, A. T.; Bowen, D. E.; Brown, S. W. (Eds.): Advances in Services Marketing Management, Vol.2. Greenwich, CT: JAI Press, pp. 65-85.

Oliver, Richard L. (1997). Satisfaction. A Behavioral Perspective on the Consumer. New York et al.: The McGraw-Hill Companies.

Oliver, Richard L. (1999). Whence Customer Loyalty? Journal of Marketing, 63 (4), pp. 33-44.

Oliver, Richard L.; Bearden, William O. (1983). The role of involvement in satisfaction processes. In: Bagozzi, R. P.; Tybout, A. M. (Eds.): Advances in consumer research. Provo, UT: Association for Consumer Research, pp. 205-255.

Oliver, Richard L.; Bearden, William O. (1985). Crossover Effects in the Theory of Reasoned Action. A Moderating Influence Attempt. Journal of Consumer Research, 12 (December), pp. 324-34.

Oshikawa, SAdomi (1968). The Theory of Cognitive Dissonance and Experimental Research. Journal of Marketing Research, 5, pp. 429-430.

o. V. (2000). Der Markt der Energieversorgung. Daten, Fakten, Trends. Reihe FOCUS Marktanalysen, Neuauflage Juni 2000. München: Focus-Magazin.

Parasuraman, Anantharanthan; Zeithaml, Valarie A.; Berry, Leonard L. (1988). SERVQUAL. A Multiple-Item Scale for Measuring Consumer Perceptions of Service Quality. Journal of Retailing, 64 (1), pp. 12-40.

Parasuraman, Anantharanthan; Zeithaml, Valerie A.; Berry, Leonard L. (1985). A Conceptual Model of Service Quality and Its Implications for Future Research. Journal of Marketing, 49, pp. 41-50.

Parasuraman, Anantharanthan; Zeithaml, Valerie A.; Berry, Leonard L. (1994). Reassessment of Expectations as a Comparison Standard in Measuring Service Quality. Implications for Further Research. Journal of Marketing, 58, pp. 111-124.

PARK, C. Whan; Mittal, Banwari (1985). A Theory of Involvement in Consumer Behavior. Problems and Issues. In: SHETH, JAGDish N. (Ed.): Research in Consumer Behavior, volume 1. Greenwich, CT: JAI Press, pp. 201-231.

Patterson, Paul G. (1993). Expectations and Product Performance as Determinants of Satisfaction for a High-Involvement Purchase. Psychology \& Marketing, 10 (5), pp. 449-465.

Patterson, Paul G.; Smith, Tasman (2003). A Cross-cultural Study of Switching Barriers and Propensity to Stay with Service Providers. Journal of Retailing, 79, pp. 107-120.

Payne, Adrian; Frow, Pennie (1997). Relationship Marketing. Key Issues for the Utility Sector. Journal of Marketing Management, 13, pp. 463-477. 
Perdue, Richard R.; Long, Patrick T.; Kang, Yong Soon (1999). Boomtown Tourism and Resident Quality of Life: The Marketing of Gaming to Host Community Residents. Journal of Business Research, 44, pp. 165-177.

Peter, J. Paul (1979). Reliability. A Review of Psychometric Basics and Recent Marketing Practices. Journal of Marketing Research, 16, pp. 6-17.

Peter, J. Paul (1981). Construct Validity. A Review of Basic Issues and Marketing Practices. Journal of Marketing Research, 18, pp. 133-145.

Peter, J. Paul; Churchill, Gilbert A. (1986). Relationships among Research Design Choices and Psychometric Properties of Rating Scales. A Meta-Analysis. Journal of Marketing Research, 23, pp. 1-10.

Peter, J. Paul; Olson, Jerry (1994). Consumer Behavior. Burr Ridge et al., $4^{\text {th }}$ edition.

Peter, J. Paul; Olson, Jerry C. (1999). Consumer Behavior and Marketing Strategy. Boston at al.: Irwin/McGraw-Hill, $5^{\text {th }}$ edition.

Peter, Sibylle Isabelle (1999). Kundenbindung als Marketingziel. Wiesbaden: Gabler, 2. Auflage.

Peterson, Robert A.; Albaum, Gerald; Beltramini, Richard F. (1985). A Meta-Analysis of Effect Sizes in Consumer Behavior Experiments. Journal of Consumer Research, 12, pp. 97-103.

Peterson, Robert A.; Wilson, William R. (1992). Measuring Customer Satisfaction. Fact and Artifact. Journal of the Academy of Marketing Science, 20, pp. $61-71$.

Petty, Richard E.; Cacioppo, John T. (1979). Issue Involvement Can Increase or Decrease Persuasion by Enhancing Message-Relevant Cognitive Responses. Journal of Personality and Social Psychology, 37 (10), pp. 1915-1926.

Petty, Richard E.; Cacioppo, John T.; Schumann, David (1983). Central and peripheral routes to advertising effectiveness. The moderating role of involvment. Journal of Consumer Research, 10, pp. 135-146.

Petty, Richard E.; Goldman, Rachel; Cacioppo, John T. (1981). Personal Involvement as a Deteminant of Argument-based Persuasion. Journal of Personality and Social Psychology, 41 (5), pp. 847-855.

Phelps, Edmund S.; Winter, Sidney G. (1970). Optimal Price Policy under Atomistic Competition. In: Phelps, Edmund S. ET AL. (Ed.): Microeconomic Foundations of Employment and Inflation Theory. New York: Norton, pp. 309-337.

Pimpl, Roland (1999). Energiemarkt-Studien im Wechselstrom. Untersuchungen kommen bei Erforschung von Markenbekanntheit und Wechselbereitschaft zu unterschiedlichen Resultaten / Yellow Strom steht immer vorne. Horizont, 7 (48), S. 32.

Ping, Robert A. (1990). Responses to Dissatisfaction in Buyer-Seller-Relationships. PhD Thesis, University of Cincinnati, Cincinnati.

Ping, Robert A. (1993). The Effects of Satisfaction and Structural Constraints on Retailer Exiting, Voice, Loyalty, Opportunism, and Neglect. Journal of Retailing, 69, pp. 320-352.

Pirovsky, Wilfriede (1999). Der Energiemarkt im Umbruch: Kundenbedürfnisse erfassen, um Kunden zu halten. Planung und Analyse, 26 (4), S. 36-40. 
Plinke, Wulff (1997). Bedeutende Kunden. In: Kleinaltenkamp, Michael; PlinKE, WulfF (Hrsg.): Geschäftsbeziehungsmanagement. Berlin et al.: Springer, S. 113159.

Plinke, WulfF (2000). Grundlagen des Marktprozesses. In: Kleinaltenkamp, MiChael; Plinke, Wulff (Hrsg.): Technischer Vertrieb. Berlin: Springer, S. 3-98.

Porter, Michael E. (1980). Competitve Strategy. Techniques for Analyzing Industries and Competitors. New York: The Free Press.

RATChFord, Brian T. (1982). Cost-Benefit Models for Explaining Consumer Choice and Information Seeking Behavior. Management Science, 28, pp. 197-212.

RATCHFORD, BRIAN T. (1987). New insights about the FCB grid. Journal of Advertising Research, 27, pp. 24-38.

ReichHeld, Frederick F. (1993). Loyalty-Based Management. Harvard Business Review, 71 (March-April), pp. 64-73.

Reichheld, Frederick F.; Aspinall, Keith (1994). Building High-Loyalty Business Systems. Journal of Retailing, 15 (4), pp. 21-29.

Reichheld, Frederick F.; SAsser, Jr., W. Earl (1990). Zero Defections. Quality Comes to Service. Harvard Business Review, pp. 105-111.

Reinartz, Werner; Krafft, Manfred; Hoyer, Wayne D. (2004). The Customer Relationship Management Process. Its Measurement and Impact on Performance. Journal of Marketing Research, 41 (3), pp. 293-305.

ReineCKe, Jost (1999). Interaktionseffekte in Strukturgleichungsmodellen mit der Theorie des geplanten Verhaltens. Multiple Gruppenvergleiche und Produktterme mit Latenten Variablen. ZUMA-Nachrichten, 23 (45), S. 88-114.

Richins, Marsha L.; Bloch, Peter H. (1991). Post-Purchase Product Satisfaction: Incorporating the Effects of Involvement and Time. Journal of Business Research, 24, pp. $145-158$.

Rindskopf, David (1984). Using Phantom and Imaginery Latent Variables to Parameterize Constraints in Linear Structural Models. Psychometrika, 49, pp. 37-47.

Rodríguez, Carlos M.; Wilson, David T. (2002). Relationship Bonding and Trust as a Foundation for Commitment in U.S.-Mexican Strategic Alliances: A Structural Equation Modeling Approach. Journal of International Marketing, 10 (4), pp. 53-76.

Roemer, Ellen (2004). Rigidity versus Flexibility in Buyer-Seller Relationships. unveröffentlichtes manuskript, Marketing Group, School of Management, University of Bradford, Bradford, UK.

Rossiter, John R. (2002). The C-OAR-SE Procedure for Scale Development in Marketing. International Journal of Research in Marketing, 19, pp. 305-335.

Rusbult, Caryl E. (1980). Commitment and Satisfaction in Romantic Associations: A Test of the Investment Model. Journal of Experimental Social Psychology, 16, pp. $172-186$.

Rust, Roland T.; Oliver, Richard L. (1994). Service quality. Insights and Managerial Implications from the Frontier. In: Rust, Roland T.; Oliver, Richard L. (Eds.): Service Quality. New Directions in Theory and Practice. London: Sage, pp. 1-19. 
Rust, Roland T.; Zahorik, Anthony J. (1993). Customer Satisfaction, Customer Retention, and Market Share. Journal of Retailing, 69 (2), pp. 193-215.

Rust, Roland T.; Zahorik, Anthony J.; Kleinigham, Timothy L. (1995). Return of Quality (ROQ). Marketing Service Quality Financially Accountable. Journal of Marketing, 59, pp. 58-70.

Ryan, Michael J. (1978). An Examination of an Alternative Form of the Behavioral Intention Model's Normative Component. In: Hunt, H. KeITH (Ed.): Advances in Consumer Research Vol., pp. 283-289. Ann Arbor, MI: Association for Consumer Research.

Ryan, Michael J.; Bonfield, Edward H. (1975). The Fishbein Extended Model and Consumer Behavior. Journal of Consumer Research, 2, pp. 118-136.

Schlesinger, Harris; Schulenburg, J.-Matthias Graf von Der (1995). Consumer Information and Decisions to Switch Insurers. Journal of Risk and Insurance, 62, pp. 591-615.

Schmalensee, Richard (1978). A Model of Advertising and Product Quality. The Journal of Political Economy, 86 (3), pp. 485-503.

Schmalensee, Richard (1982). Production Differentiation Advantages of Pioneering Brands. American Economic Review, 72, pp. 349-365.

Schmid, John; Leiman, John M. (1957). The development of hierarchical factor solutions. Psychometrika, 22, pp. 53-61.

Schoenheit, Ingo; NiedergesÄss, Ulrike (1999). Kundenbindung bei privaten Haushalten. Energiewirtschafliche Tagesfragen, 49 (7), S. 464-467.

Schütze, Roland (1992). Kundenzufriedenheit. After-Sales-Marketing auf industriellen Märkten. Wiesbaden: Gabler.

SeIferth, Thorsten (1999). Kundenorientierung bei EVU. Begriff. Instrumente. Eignung. Energiewirtschaftliche Tagesfragen, 49 (6), S. 383-388.

Seilheimer, Christian; Herrmann, Andreas; Huber, Frank (2002). Kundenzufriedenheit und Kundenloyalität im Lichte des Regret. Wirtschaftswissenschaftliches Studium, 31 (2), S. 80-84.

Selten, Reinhard (1965). Spieltheoretische Behandlung eines Oligopolmodells mit Nachfrageträgheit. Zeitschrift für die Gesamte Staatswissenschaft, 121 (4), S. 301-324 und 667-689.

Shaffer, Terry Root; Sherrell, Daniel L. (1997). Consumer Satisfaction with Health-Care Services: The Influence of Involvement. Psychology \& Marketing, 14 (3), pp. 261-285.

Sharma, Neeru; Patterson, Paul G. (2000). Switching Costs, Alternative Attractiveness and Experience as Moderators of Relationship Commitment in Professional Consumer Services. International Journal of Service Industry Management, 11 (5), pp. 470-490.

Sheppard, Blair H.; Hartwick, Jon; Warshaw, Paul R. (1988). The Theory of Reasoned Action. A Meta-Analysis of Past-Research with Recommendations for Modifications and Future Research. Journal of Consumer Research, 15 (December), pp. $325-343$. 
Sherif, Muzafer; Cantril, Hadley (1947). The Psychology of Ego-involvement. New York: John Wiley \& Sons.

Sheth, Jagdish N.; Parvatiyar, Atul (1995). Relationship Marketing in Consumer Markets. Antecedents and Consequences. Journal of the Academy of Marketing Science, 23, pp. 255-271.

SILZER, ERICH (1959). Die Problematik der westdeutschen Elektrizitätswerke unter besondererer Berücksichtigung der Stromversorgung und Strompreisgestaltung für industrielle Sonderabnehmer. Dissertation, Universität Nürnberg, Nürnberg.

Simon, Hermann; Homburg, Christian (1995). Marktbarrieren. In: Tietz, B.; KöHleR, R.; Zentes, J. (Hrsg.): Handwörterbuch des Marketing. Stuttgart: SchäfferPoeschel, 2. Auflage, S. 1744-1756.

Simon, Hermann; Homburg, Christian (1997). Kundenzufriedenheit als strategischer Erfolgsfaktor. Einführende Überlegungen. In: Simon, Hermann; HomburG, Christian (Hrsg.): Kundenzufriedenheit. Konzepte, Methoden, Erfahrungen. Wiesbaden: Gabler, 2. Auflage, S. 17-29.

Singh, JAGDIP (1991). Understanding the Structure of Consumers' Satisfaction Evaluations of Service Delivery. Journal of the Academy of Marketing Science, 19, pp. 223-244.

Snyder, Mark (1982). When Believing Means Doing: Creating Links between Attitudes and Behavior. In: Zanna, Mark P.; Higgins, E. Tory; Herman, C. Peter (Eds.): Consistency in Social Behavior. Hillsdale, NJ: Lawrence Erlbaum.

Söllner, Albrecht (1993). Commitment in Geschäftsbeziehungen. Das Beispiel Lean Production. Wiesbaden: Gabler.

Sorce, Patricia; Edwards, Kimberly (2004). Defining Business-to-Consumer Relationships: The Consumer's Perspective. Journal of Database Marketing \& Customer Strategy Management, 11 (3), pp. 255-267.

Spence, Mark T.; Brucks, Merrie (1997). The Moderating Effects of Problem Characteristics on Experts' an Novices' Judgments. Journal of Marketing Research, 34, pp. 233-247.

Stango, Victor (2002). Pricing with Consumer Switching Costs. Evidence from the Credit Card Market. The Journal of Industrial Economics, 50 (4), pp. 475-492.

Stanley, Scott M.; Markman, Howard J. (1992). Assessing Commitment in Personal Relationships. Journal of Marriage and the Family, 54, pp. 595-608.

Stauss, Bernd (1999). Kundenzufriedenheit. Marketing · Zeitschrift für Forschung und Praxis, 21 (1), S. 5-24.

Stauss, Bernd; Hentschel, Bert (1992). Messung von Kundenzufriedenheit. Merkmals- und ereignisorientierte Beurteilung von Dienstleistungsqualität. Marktforschung und Management, 36, S. 115-122.

Stauss, Bernd; Neuhaus, Patricia (1995). Das Qualitative Zufriedenheitsmodell (QZM). Diskussionsbeiträge 66, Wirtschaftswissenschaftliche Fakultät Ingolstadt.

Steenkamp, Jan-Benedict E. M. (1990). Conceptual Model of the Quality Perception Process. Journal of Business Research, 21, pp. 309-333.

Stewart, Kate (1998). An Exploration of Customer Exit in Retail Banking. International Journal of Bank Management, 16 (1), pp. 6-14. 
Stewart, Thomas A. (1997). A Satisfied Customer Isn't Enough. Fortune, 136, pp. $112-113$.

Stigler, George J. (1961). The Economics of Information. The Journal of Political Economy, 69, pp. 213-225.

Stone, Robert N.; Mason, Barry J. (1995). Attitude and Risk: Exploring the Relationship. Psychology \& Marketing, 12 (2), pp. 135-153.

Strandvik, Tore; Liljander, Veronica (1994). A comparison of episode performance and relationship performance for a discrete service. working paper, Paper presented at the $3^{\text {rd }}$ Service Marketing Workshop, Berlin, Germany.

Sturm, David; Thiry, Alain (1991). Building Customer Loyalty. Training \& Development Journal, pp. 34-36.

Sutton, John (1980). A Model of Stochastic Equilibrium in a Quasi-Competitive Industry. Review of Economic Studies, 47, pp. 705-722.

Swan, John E.; Trawick, Frederick I. (1979). Testing an extended concept of consumer satisfaction. In: DAY, RALPh L.; Hunt, H. KeITH (Eds.): New dimensions of consumer satisfaction and complaining behavior. Bloomington, IN: University of Indiana School of Business, pp. 56-61.

Szymanski, David M.; Henard, David H. (2001). Customer Satisfaction. A MetaAnalysis of the Empirical Evidence. Journal of the Academy of Marketing Science, 29 (1), pp. 16-35.

Taylor, Dalmas A.; Altman, Irwin (1973). Social Penetration: The Development of Interpersonal Relationships. New York: Holt, Rinehart \& Winston.

Taylor, Steven A.; Baker, Thomas L. (1994). An Assessment of the Relationship Between Service Quality and Customer Satisfaction in the Formation of Consumers' Purchase Intentions. Journal of Retailing, 70 (2), pp. 163-178.

Teas, Kenneth R. (1993). Expectations, Performance Evaluation and Consumers' Perceptions of Quality. Journal of Marketing, 57, pp. 18-34.

Thau, Stefan; Bennett, Rebecca J.; Stahlberg, Dagmar; Werner, Jon M. (2004). Why Should I Be Generous When I Have Valued and Accessible Alternatives? Alternative Exchange Partners and OCB. Journal of Organizational Behavior, 25, pp. 607-626.

Thibaut, John W.; Kelley, Harold H. (1959). The Social Psychology of Groups. New York/London/Sydney: Wiley.

TÖPfER, ARMin (2001). Organisierte Interessenvertretung der Tarifkunden/Verbraucher. In: Kahmann, Martin; KÖNIG, Siegfried (Hrsg.): Wettbewerb im liberalisierten Strommarkt. Regeln und Techniken. Berlin et al.: Springer, S. 191-204.

Töpfer, Armin; Wieder, Martin (1996). Effiziente Kundenbindungsprogramme. In: TöPfER, Armin (Hrsg.): Kundenzufriedenheit messen und steigern. Neuwied/Kriftel/ Berlin: Luchterhand, S. 303-342.

Triandis, Harry C. (1980). Values, Attitudes, and Interpersonal Behavior. In: Howe, Jr., Herbert E.; PAge, Monte M. (Eds.): Beliefs, Attitudes, and Values. Nebraska Symposium on Motivation 1979, 2\%. Lincoln/London: University of Nebraska Press, pp. 195-259.

Ungern-Sternberg, T. R. von (1982). Equilibrium Prices in a Model with Differen- 
tiated Goods and Search. Journal of Institutional and Theoretical Economics- Zeitschrift für die gesamte Staatswissenschaft, 138, S. 22-35.

Ungern-Sternberg, Thomas R. von (1984). Zur Analyse von Märkten mit unvollständiger Nachfrageinformation. Berlin/Heidelberg/New York: Springer.

Urbany, Joel E. (1986). An Experimental Examination of the Economics of Information. Journal of Consumer Research, 13, pp. 257-271.

VDEW (2000). Kundenzufriedenheit bei Haushaltskunden. Ergebnisbericht des VDEWKundenfokus Haushalte 2000. Frankfurt am Main/Heidelberg: VWEW-Energieverlag.

VDEW (2001). Kundenzufriedenheit bei Haushaltskunden. Ergebnisbericht des VDEWKundenfokus Haushalte 2001. Frankfurt am Main/Heidelberg: VWEW-Energieverlag.

VDEW (2002a). Kundenzufriedenheit bei Haushaltskunden. Ergebnisbericht des VDEWKundenfokus Haushalte 2002. Frankfurt am Main/Heidelberg: VWEW-Energieverlag.

VDEW (2002b). Stromkunden nutzen neue Angebote. Meldungen aus Politik und Wirtschaft vom 28.01.02, http://www. strom.de.

VDEW (2003). Kundenzufriedenheit bei Haushaltskunden. Ergebnisbericht des VDEWKundenfokus Haushalte 2003. Frankfurt am Main/Heidelberg: VWEW-Energieverlag.

Viard, V. Brian (2002). Do Switching Costs Make Markets More or Less Competitive? The Case of 800-Number Portability. Stanford, CA: Stanford University.

Vollmer, InGRID (2002). Die Loyalität unzufriedener Kunden. Determinanten und Implikationen. Wiesbaden: Deutscher Universitäts-Verlag.

Wagner, Oliver; Kristof, Kora (2002). Energienahe, ökoeffiziente Dienstleistungen kommunaler Energieversorger im wettbewerblichen Umfeld. In: BosCH, GERHARD; Hennicke, Peter; Hilbert, Josef; Kristof, Kora; Scherhorn, Gerhard (Hrsg.): Die Zukunft von Dienstleistungen. Auswirkungen auf Arbeit, Umwelt und Lebensqualität. Frankfurt/New York: Campus, S. 422-452.

Warshaw, Paul R. (1980a). A New Model for Predicting Behavioral Intentions. An Alternative to Fishbein. Journal of Marketing Research, 17 (2), pp. 153-72.

Warshaw, Paul R. (1980b). Predicting Purchase and Other Behaviors from General and Contextually Specific Intentions. Journal of Marketing Research, 17 (1), pp. 26-33.

Wathne, Kenneth H.; Biong, Harald; Heide, Jan B. (2001). Choice of Supplier in Embedded Markets: Relationship and Marketing Program Effects. Journal of Marketing, 65 (2), pp. 54-66.

Weiber, Rolf; Adler, Jost (2003). Der Wechsel von Geschäftsbeziehungen beim Kauf von Nuzungsgütern. Das Beispiel Telekommunikation. In: Rese, Mario; Söllner, Albrecht; Utzig, B. Peter (Hrsg.): Relationship Marketing. Standortbestimmung und Perspektiven. Berlin/Heidelberg: Springer, S. 71-103.

Weinberg, Peter (1977). Die Produkttreue der Konsumenten. Wiesbaden: Gabler.

Weinberg, Peter (1987). Markentreue und Markenwechsel. In: Hoyos, CARL Graf von; Kroeber-Riel, Werner; Rosenstiel, Lutz von (Hrsg.): Wirtschaftspsychologie in Grundbegriffen. Gesamtwirtschaft - Markt - Organisation - Arbeit. München/Weinheim: Psychologie-Verlags-Union, S. 162-169.

Weiss, Allen M.; Anderson, Erin (1992). Converting From Independent to Employee Salesforces. The Role of Perceived Switching Costs. Journal of Marketing Research, 29, pp. 101-115. 
Westbrook, Robert A.; Reilly, Michael D. (1983). Value Percept Disparity. An Alternative to the Disconfirmation of Expectations Theory of Consumer Satisfaction. In: Bagozzi, Richard P.; Tybout, Alice M. (Eds.): Advances in Consumer Research. Ann Arbor: MI: Association for Consumer Research, pp. 256-261.

Wied-NebBeling, Susanne (1993). Markt- und Preistheorie. Berlin/Heidelberg/New York: Springer.

Wiedmann, Klaus-Peter; Walsh, Gianfranco (2003). Der Einfluss der Zufriedenheit auf die Wechselbereitschaft von privaten Gaskunden. Empirische Analyse und Implikationen. Zeitschrift für Energiewirtschaft, 27 (3), S. 225-233.

Wilkie, William L. (1994). Consumer Behavior. New York et al.: John Wiley \& Sons, $3^{\text {rd }}$ edition.

Wold, Herman (1966). Non-linear Estimation by Iterative Least Squares Procedures. In: David, F. N. (Ed.): Research papers in statistics. London/New York/Sydney: John Wiley \& Sons, pp. 411-444.

Wold, Herman (1969). Nonlinear Iterative Partial Least Squares (NIPALS) Estimation Procedures. Bulletin of the International Statistical Institute, 43, pp. 29-51.

Wold, Herman (1973). Nonlinear Iterative Partial Least Squares (NIPALS) Modelling. Some Current Developments. In: Krishnaiah, Paruchuri R. (Ed.): Proceedings of the $3^{\text {rd }}$ International Symposium on Multivariate Analysis, pp. 383-407. Dayton, OH.

Wold, Herman (1980). Model Construction and Evaluation When Theoretical Klnowledge is Scarce. Theory and Application of Partial Least Squares. In: KMEnTA, JAN; Ramsey, James B. (Eds.): Evaluation of Econometric Models. New York: Academic Press, pp. 47-74.

Wold, Herman (1982). Soft Modeling. The Basic Design and Some Extensions. In: Jöreskog, Karl G.; Wold, Herman (Eds.): Systems under Indirect Observation. Causality, Structure, Prediction. Part I. Amsterdam/New York/Oxford: NorthHolland, pp. 1-54.

Woodside, Arch G.; Frey, Lisa L.; Daly, Robert T. (1989). Linking Service Quality, Customer Satisfaction, and Behavioral Intention. Journal of Health Care Marketing, 9 (4), pp. 5-17.

Yang, Zhilin; Peterson, Robin T. (2004). Customer Perceived Value, Satisfaction, and Loyalty. The Role of Switching Costs. Psychology \& Marketing, 21 (10), pp. 799-822.

Yi, Yougae (1990). A Critical Review of Consumer Satisfaction. In: Zeithaml, VAlerie A. (Ed.): Review of Marketing. Chicago: American Marketing Association, pp. $68-123$.

Zaichkowsky, Judith L. (1985). Measuring the Involvement Construct. Journal of Consumer Research, 12 (September), pp. 341-352.

Zaichkowsky, Judith L.; Sood, James H. (1988). A Global Look at Consumer Involvement and Use of Products. International Marketing Review, 6 (1), pp. 20-34.

Zeithaml, Valerie A. (2000). Service Quality, Profitability, and the Economic Worth of Customers. What we Know and what we need to Learn. Journal of the Academy of Marketing Science, 28, pp. 67-85.

Zeithaml, Valerie A.; Berry, Leonard L.; Parasuraman, Anantharanthan 
(1991). The Nature and Determinants of Customer Expectations of Service. working paper MSI Report No. 91-113, Marketing Science Institute, Cambridge, MA.

Zeithaml, Valerie A.; Berry, Leonard L.; Parasuraman, Anantharanthan (1996). The Behavioral Consequences of Service Quality. Journal of Marketing, 60, pp. 31-46.

Zezelu, Gordana (2000). Customer-Lifetime-Value-Management. In: Hofmann, Markus; Mertiens, Markus (Hrsg.): Customer-Lifetime-Value-Management. Kundenwert schaffen und erhöhen. Konzepte, Strategien, Praxisbeispiele. Wiesbaden: Gabler, S. 31-42.

Zinnbauer, Markus (2001). Cross-Selling-Potenziale bei Energieversorgungsunternehmen durch Bundling. Zeitschrift für Energiewirtschaft, 25 (4), S. 243-252. 


\section{Anhang}

\section{A.1 Fragebogen}

\section{Einleitungstext}

Guten Tag, mein Name ist [Name] $]^{1}$. Ich bin Student/in an der Universität Kaiserslautern. Wir führen gemeinsam mit [Anbieter $]^{2}$ eine Umfrage zum Thema Stromversorgung durch. Ihre Meinung dazu ist uns sehr wichtig. Hätten Sie ein paar Minuten Zeit? ${ }^{3}$ Sind Sie in Ihrem Haushalt an der Entscheidung über die Wahl des Stromversorgers beteiligt?4

\section{Zustimmungsfragen}

Ich lese Ihnen jetzt einige allgemeine Aussagen zum Strommarkt vor. Vergeben Sie bitte Punkte zwischen 1 und 5. Je mehr Punkte sie vergeben, desto eher stimmen sie der Aussage zu. 5 bedeutet „stimme voll und ganz zu“, 1 bedeutet „stimme überhaupt nicht zu“ und 3 wäre dementsprechend „teils teils“. Haben Sie noch Fragen zum Ablauf? Dann geht's los.

- Ich interessiere mich für die verschiedenen Angebote im Strommarkt.

- Die Informationen von meinem Versorger sind glaubwürdig.

- Seitdem man seinen Stromanbieter frei wählen kann, achte ich auch verstärkt auf die Leistungen meines eigenen Versorgers.

- Bei einem Umzug in eine andere Gegend würde ich wohl wieder zum dort ansässigen Versorger gehen, da mir die Suche nach Alternativen zu mühsam ist.

- Mein Stromversorger hält seine Versprechen ein und kommt seinen Verpflichtungen nach.

- Ich denke, dass man aus reiner Gewohnheit bei seinem Versorger bleibt.

- Ich interessiere mich für Preisvergleiche zwischen verschiedenen Stromanbietern.

- Sollte mich jemand zu Stromanbietern um Rat fragen, so könnte ich ihm Auskunft geben.

- Ich halte meinen Stromversorger für vertrauenswürdig.

1 An dieser Stelle fügte jeder Interviewer seinen Namen ein

2 Hier wurde der jeweilige regionale Stromanbieter eingesetzt.

3 Auf Nachfrage wurde als Zeitrahmen 10-15 Minuten genannt.

4 Das Interview wurde bei Verneinung dieser Frage abgebrochen 
- Die Wahl des Stromversorgers ist eine folgenschwere Entscheidung, die man sich gut überlegen sollte.

- Beim Wechsel zu einem neuen Stromversorger wäre ich immer ein wenig unsicher.

- Es ist viel Arbeit, sich über Stromtarife zu informieren.

- Die etwaige Unsicherheit mancher Kunden nutzt mein Versorger zu seinem Vorteil aus.

- Solange ich meinen Strom problemlos bekomme, gibt es für mich keinen Anlass, über einen neuen Anbieter nachzudenken.

- Bei der Auswahl eines neuen Stromversorgers kann man sich leicht irren.

- Die Vielzahl von Anbietern und Tarifen macht es schwer, einen besseren Anbieter zu finden.

- Bei der Wahl des falschen Stromanbieters hat man sehr viel zu verlieren.

- Um den Stromanbieter zu wechseln, müsste man sich vorher genau erkundigen.

- Ich glaube, man ist einfach zu träge, um den Stromanbieter zu wechseln.

- Den günstigsten Stromanbieter oder -tarif für sich herauszufinden kostet sehr viel Zeit.

- Mein Anbieter ist imstande, alle Probleme bei der Stromversorgung zuverlässig zu beheben.

- Ich konnte mich bislang einfach noch nicht dazu durchringen, mich mit der Auswahl eines neuen Stromanbieters zu beschäftigen.

- Im Vergleich mit anderen Stromversorgern habe ich bei meinem Anbieter geringere Kosten.

- Die neuen Anbieter im Strommarkt wecken meine Aufmerksamkeit.

- Ein Anbieterwechsel verursacht mehr Mühe als man zunächst annimmt.

- Da mir sehr viel an meinem jetzigen Stromversorger liegt, kommt ein Wechsel aus rein preislichen Gründen für mich nicht in Frage.

- Verglichen mit anderen Stromversorgern bietet mein Anbieter besonders gute Leistungen.

- Dadurch dass man Teil einer großen Versorgungsgemeinschaft ist, fühlt man sich geborgen.

- Ich stehe zu meinem Anbieter, da er ein Teil unserer Region ist.

- Da sich mein aktueller Stromversorger stets um mich als Kunden bemüht hat, wäre es nicht fair, ihn nur des Geldes wegen gegen einen anderen Anbieter auszutauschen.

- Ich fühle mich meinem Anbieter gegenüber sehr verbunden, da er für die Sicherheit meiner Grundversorgung steht.

- Ich glaube, dass ich bei anderen Stromanbietern mehr für mein Geld erhalte. 
- Ich glaube, dass man einfach zu bequem ist, sich um Alternativen für die Stromversorgung zu kümmern.

- Mein Stromversorger bietet im Vergleich mit anderen Anbietern ein gutes PreisLeistungs-Verhältnis.

- Ein Anbieterwechsel ist mit einem großen Aufwand verbunden.

- Die Wahl eines Stromanbieters ist schon eine langfristige Sache.

- Andere Stromversorger wären mir von größerem Nutzen als mein derzeitiger Anbieter.

- Den Stromanbieter zu wechseln ist sehr umständlich.

- Insgesamt gesehen ist ein Stromanbieterwechsel eine anstrengende Sache.

- Mein Versorger ist auf jeden Fall in der Lage, meine Stromversorgung auch in Zukunft sicherzustellen.

- Über andere Stromanbieter und -tarife sollte man sich dann informieren, wenn man einmal so richtig viel Zeit hat.

- Ich habe die feste Absicht, meinen Stromanbieter zu wechseln.

[Es schließen sich einige proprietäre Fragen des Anbieters an, die nicht abgedruckt sind.]

\section{Zufriedenheitsfragen}

Bei den nächsten Fragen geht es um Ihre Zufriedenheit. Vergeben Sie bitte Punkte zwischen 1 und 5. Bitte vergeben Sie umso mehr Punkte, je zufriedener Sie sind. 5 bedeutet „sehr zufrieden“, 1 bedeutet „,sehr unzufrieden“ und 3 wäre dementsprechend „teils teils".

- Wie zufrieden sind Sie insgesamt mit [Anbieter]?

- Wie zufrieden sind Sie damit, wie [Anbieter] Ihre Erwartungen erfüllen?

- Stellen Sie sich nun bitte einen idealen Stromanbieter vor, der allen ihren Wünschen entspricht. Wie zufrieden sind Sie dann noch mit [Anbieter]?

[Filterfrage:] Sind Sie zu einem anderen Stromanbieter gewechselt?

[Falls ja, stelle die folgenden drei Fragen:]

Bitte vergeben Sie nun Punkte für die Zufriedenheit mit Ihrem anderen Anbieter.

- Wie zufrieden sind Sie insgesamt mit Ihrem anderen Stromanbieter?

- Wie zufrieden sind Sie damit, wie Ihr anderer Versorger Ihre Erwartungen erfüllt?

- Stellen Sie sich nun bitte einen idealen Stromanbieter vor, der allen ihren Wünschen entspricht. Wie zufrieden sind Sie dann noch mit Ihrem anderen Anbieter?

\section{Wechselwahrscheinlichkeitsfrage}

Wie wahrscheinlich ist es, dass Sie Ihrem aktuellen Stromanbieter in absehbarer Zeit (d. h. in den kommenden 6-12 Monaten bzw. zum Vertragsende) kündigen werden? [Antwortoptionen: „unter keinen Umständen“, „sehr unwahrscheinlich“, „vielleicht“, „gut möglich“, „sehr wahrscheinlich, „sicher", „kann ich nicht beurteilen“]

[Es schließen sich einige proprietäre Fragen des Anbieters an, die nicht abgedruckt sind.] 


\section{Soziodemographische Fragen}

Zum Schluss benötigen wir noch einige statistische Angaben zu Ihrer Person.

In welchem Altersbereich befinden Sie sich?

[Antwortoptionen: „0-24“, „25-34“, ,35-44“, ,45-54“, ,55-64“, „>64“, ,keine Angabe“]

Wie viele Personen leben in Ihrem Haushalt?

[Antwortoptionen: „1“, ,2“, ,3“, ,4“, ,5“, ,>5“, ,keine Angabe“]

Wohnen Sie in Miete oder Eigentum?

[Antwortoptionen: „Miete“, „Eigentum“, „keine Angabe“]

Sind Sie Mitglied in einem Sportverein?

[Antwortoptionen: „Ja, nämlich: [offen]“, „Nein“, „Weiß nicht/keine Angabe“]

Inwieweit sind sie berufstätig?

[Antwortoptionen: „in Ausbildung (z.B. Schule, Lehre, Uni)“, „Angestellter“, „Beamter“, „Selbständig/Freiberufler“, „Arbeiter/Facharbeiter“, „Rentner/Pensionär“, „Hausfrau/Hausmann“, „nicht berufstätig“, „keine Angabe“]

In welchem der folgenden Bereiche liegt Ihr monatliches Haushaltsnettoeinkommen?

[Antwortoptionen: „unter 500€“, „500 bis unter 1000€“, „1000 bis unter $1500 € “, ~ „ 1500$ bis unter $2000 € “$, „2000 bis unter $2500 € “, ~ „ 2500$ bis unter $3000 € “,$, ,3000 bis unter 3500€“, „3500€und darüber", „keine Angabe“]

\section{Ende der Befragung}

Wir sind nun am Ende der Befragung angekommen. Herzlichen Dank für Ihre Bereitschaft, bei dieser Befragung teilzunehmen!

[Platz für Bemerkungen]

[Geschlecht des Befragten:]

[Interviewer:] 


\section{A.2 Korrelationsmatrix}

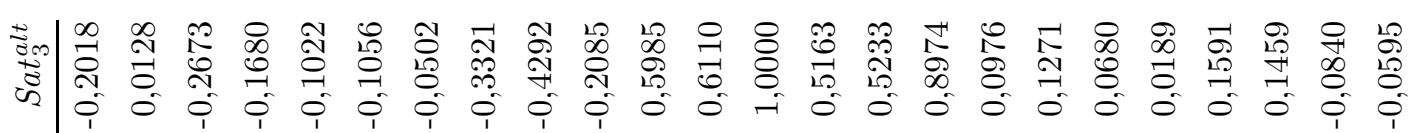

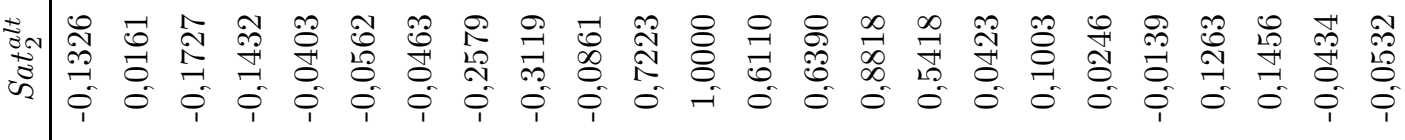

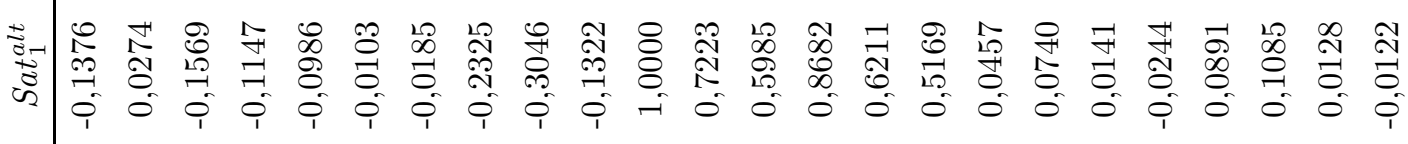

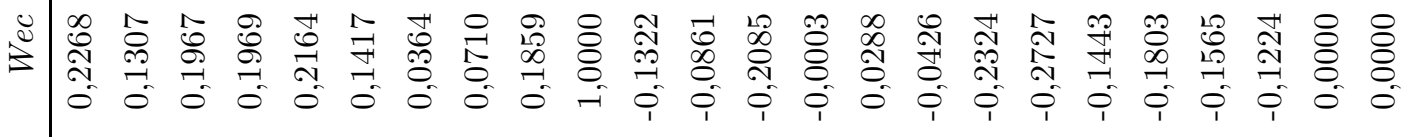

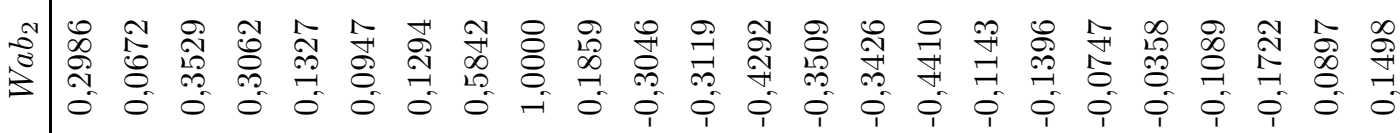

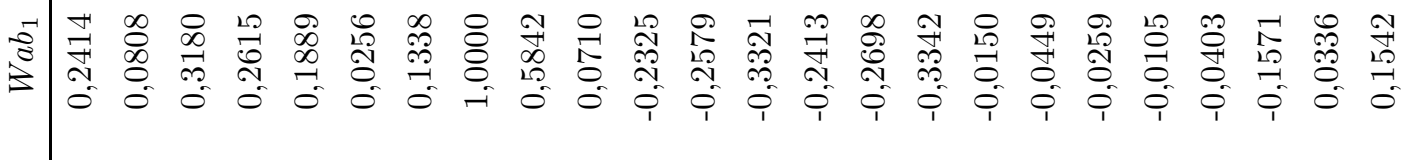

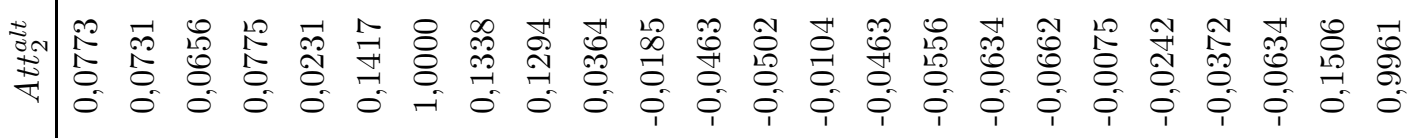

节

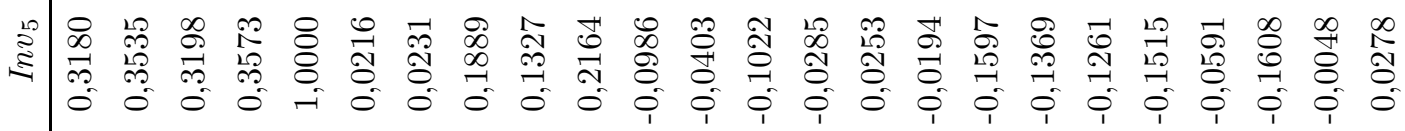

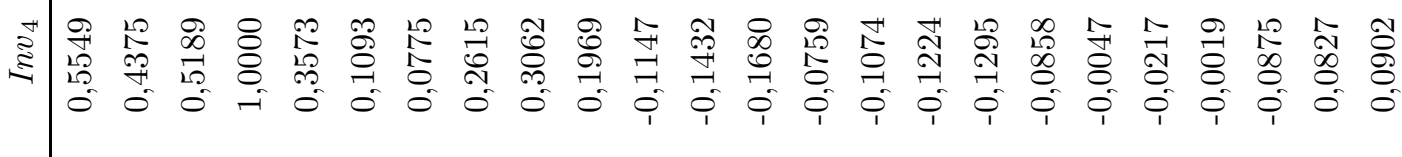

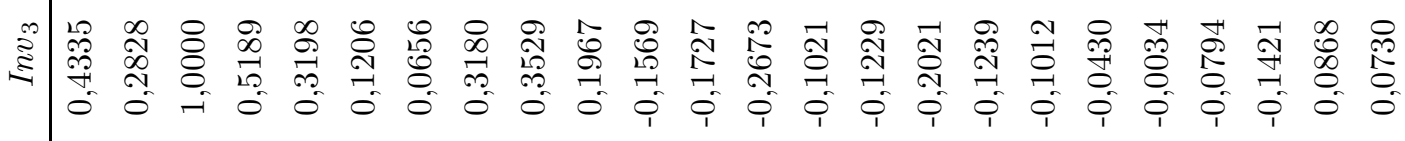

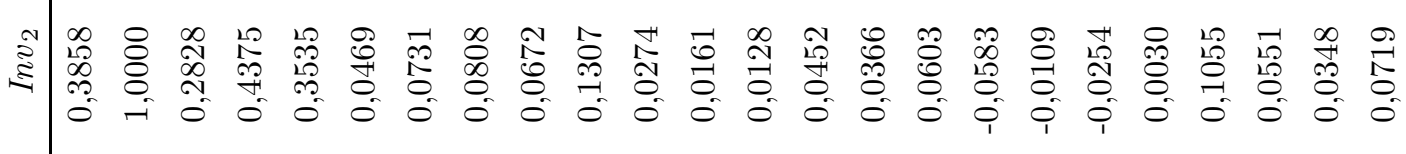

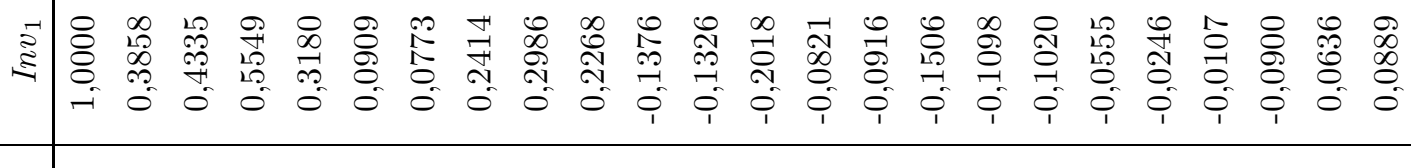

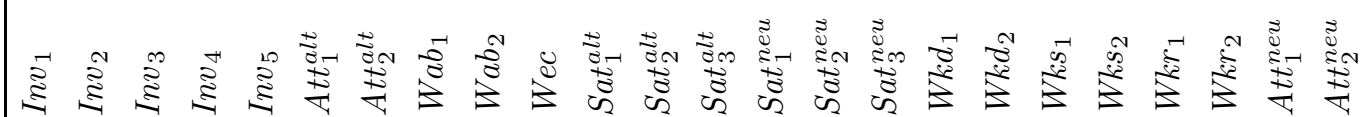


Korrelationsmatrix (Fortsetzung)

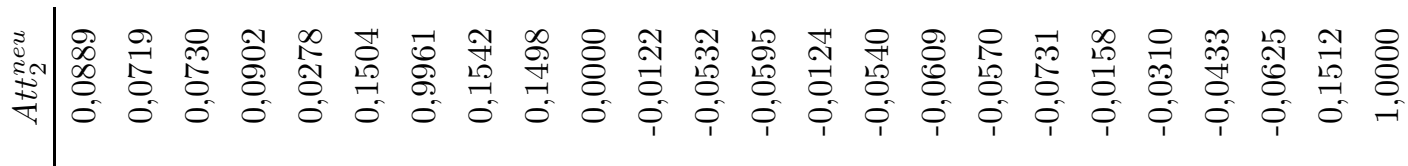

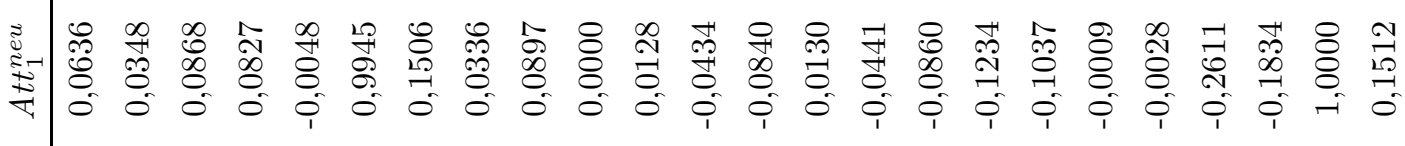

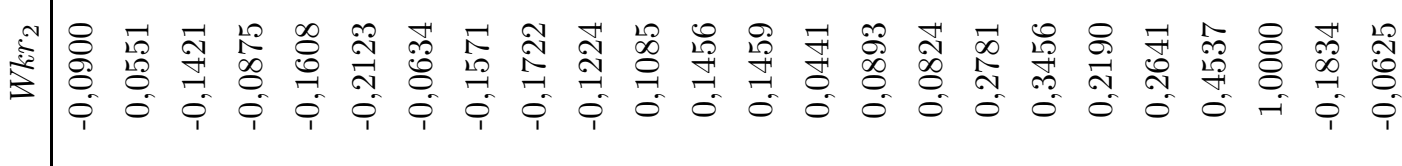

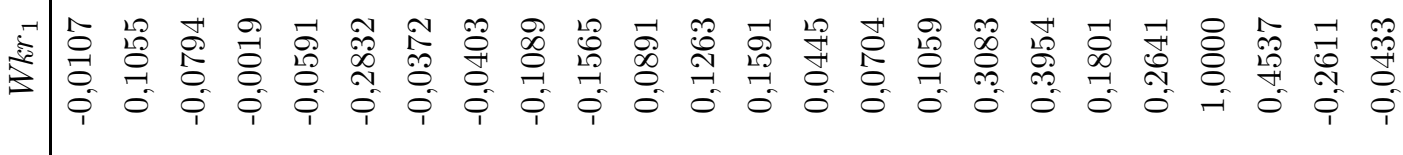

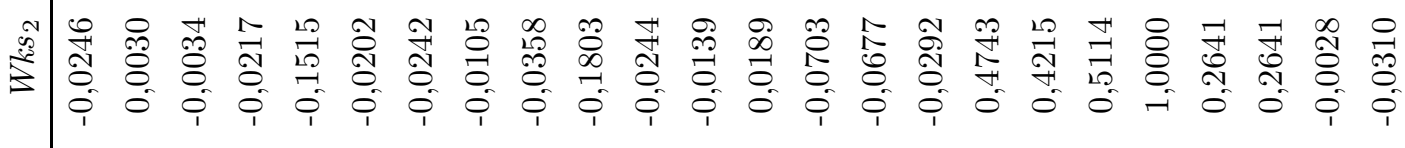

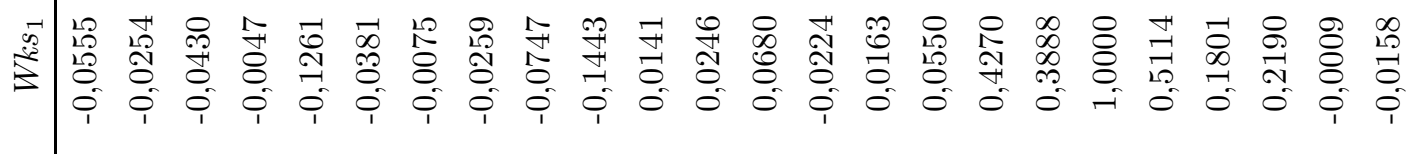

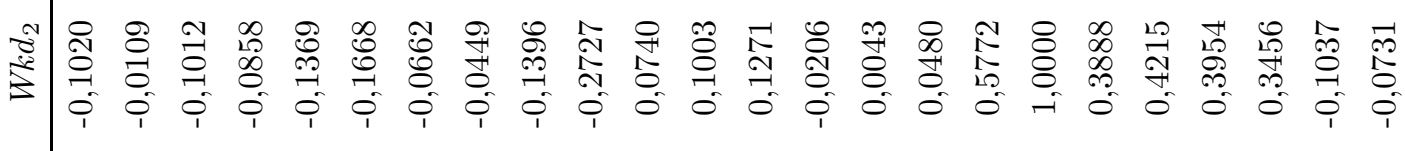

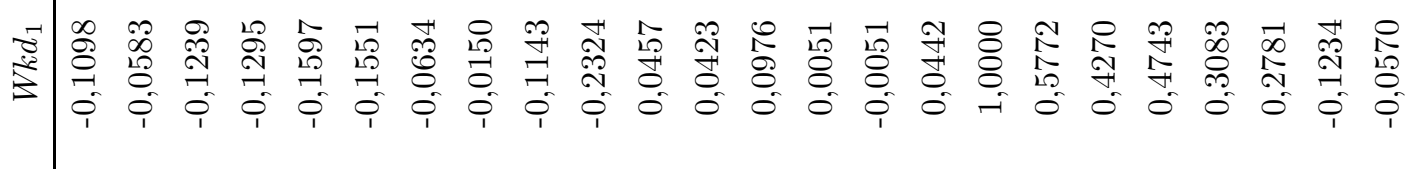

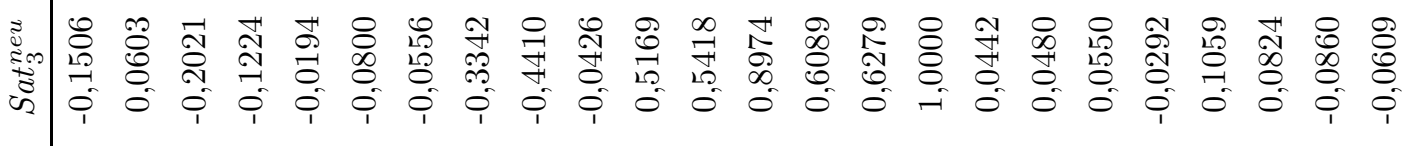

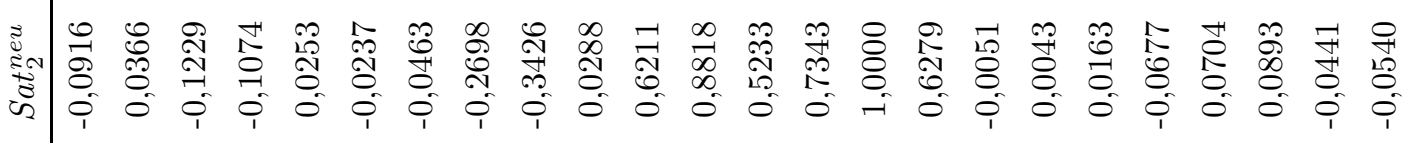

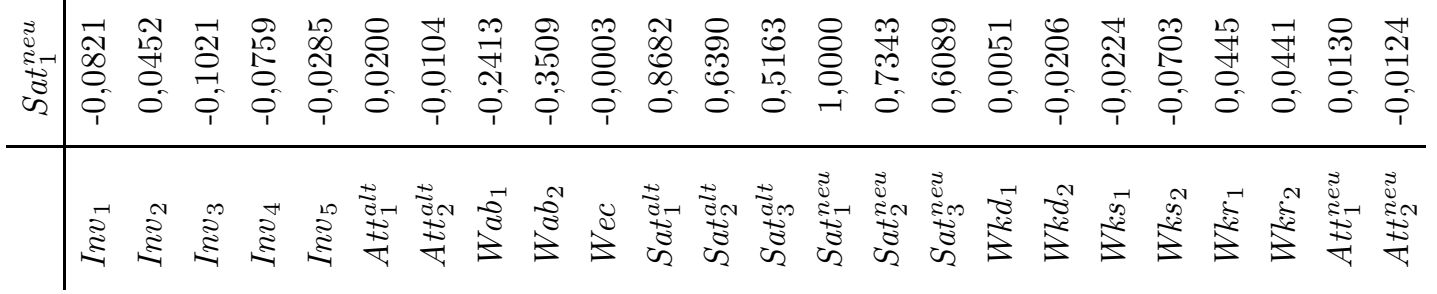




\section{A.3 Textquellen zur Trägheit}

Die folgenden auszugsweise abgedruckten Textquellen belegen, dass verschiedene Interessengruppen die Trägheit der Konsumenten als Erklärungsansatz für das gering ausgeprägte Wechselverhalten im deutschen Strommarkt ansehen. Das Dokument in Unterabschnitt A.3.1 entstammt dem Kreis der etablierten Anbieter. Das Dokument in Unterabschnitt A.3.2 ist den neuen Anbietern zuzurechnen. Das Dokument in Unterabschnitt A.3.3 lässt sich mit Verbraucherverbänden in Verbindung bringen.

\section{A.3.1 Einfluss von Trägheit aus Sicht etablierter Anbieter}

Der folgende Textauszug entstammt einem Vortragsmanuskript von Dr. Eberhard Meller, dem Hauptgeschäftsführer des VDEW. Der Vortrag mit dem Titel „Erfahrungen mit dem Wechselverhalten der Kunden im liberalisierten deutschen Strommarkt" wurde am 29. November 2002 im Rahmen des 3. NaturEnergie-Symposiums in Grenzach-Wyhlen gehalten. Der Text wurde am 10. Januar 2005 von der Internetseite des VDEW abgerufen. $[\ldots]$

Der Stromkunde ist aber kein homo oeconomicus, genauso wenig wie es die Kunden anderer Branchen sind. Mangelnde Informationen, Trägheit oder Desinteresse stehen der Auseinandersetzung der Kunden mit dem Thema Stromversorgung und mit neuen Anbietern entgegen. Dies ist nicht verwunderlich, wenn man die geringe Bedeutung der Stromkosten für die Finanzen eines Haushalts sieht. Der Anteil der Ausgaben für Energie, das heißt hier Strom und Heizung, am gesamten Budget eines durchschnittlichen Vier-PersonenHaushalts beträgt nur knapp vier Prozent. Strom allein macht etwa 1,5 Prozent aus. Für viele Haushalte ist das Thema Stromversorgung auch aus finanzieller Sicht schlicht nicht relevant.

$[\ldots]$

\section{A.3.2 Einfluss von Trägheit aus Sicht neuer Anbieter}

Der folgende Textausschnitt wurde am 10. Januar 2005 von der Internetseite des Bundesverbands Windenergie e.V. (http://www.wind-energie.de/) abgerufen. Den Text verfasste Oliver Lönker unter dem Titel „Neue Energie - März 2004“.

$[\ldots]$

„Geiz ist geil!" Am neuen Credo der Bundesbürger kommt keiner mehr vorbei - auch nicht die Ökostrom-Versorger. Spätestens beim Gedanken an den eigenen Geldbeutel scheint die 
Bereitschaft zu schwinden, für saubere Energie ein paar Euro draufzulegen. Die Wechselbilanz im Jahre sechs nach Liberalisierung der Strommärkte ist bescheiden: Nur 1,3 Prozent der deutschen Haushalte bezogen Ende 2003 ihren Strom von einem grünen Anbieter, schätzen die Autoren einer noch unveröffentlichten Studie des Instituts für Wirtschaft und Ökologie an der Universität Sankt Gallen (IWÖ-HSG). Sparsamkeit und Trägheit machen Deutschlands Stromkunden zu Wechselmuffeln. Die meisten Haushalte bleiben ihrem Regionalversorger selbst dann treu, wenn grüne Energie günstiger zu haben ist als Atom- und Kohlestrom. Als Konsequenz, kommentierte unlängst die Berliner tageszeitung, nähmen die alt eingesessenen Anbieter die Privatkunden als mündige Marktteilnehmer kaum noch ernst. Ist das Projekt „Stromwechsel für die Energiewende" gescheitert? „Die Entwicklung des Ökostrom-Marktes ist besser als sein Ruf", entgegnet der Chef von Greenpeace Energy, Robert Werner. Sein Unternehmen konnte im Jahr 2003 wie die meisten anderen grünen Anbieter ein Kundenwachstum im deutlich zweistelligen Bereich vermelden. 20.000 Abnehmer haben die Regenbogenkämpfer bis heute akquiriert und 2004 sollen es bis zu 30 Prozent mehr werden. Insgesamt haben bis Ende vergangenen Jahres 490.000 Kunden zu einem Alternativstrom-Anbieter gewechselt, schätzt das IWÖ-HSG. Tendenz: steigend.

$[\ldots]$

\section{A.3.3 Einfluss von Trägheit aus Verbrauchersicht}

Der folgende Textausschnitt entstammt einem Artikel aus der Welt am Sonntag vom 11. Februar 2001 mit dem Titel „,Schaulauf der Stromanbieter. Rund 40 private Firmen kämpfen inzwischen gegen den Hamburger Energie-Riesen HEW um Marktanteile." Verfasst wurde der Artikel von Cornelis Rattmann. Der Textausschnitt wurde am 10. Januar 2005 von der Internetseite des Ökostrom-Anbieters LichtBlick abgerufen.

$[\ldots]$

Dass so wenige Hamburger den Stromanbieter wechseln, führt Helmut Gumtau, EnergieExperte der Verbraucherzentrale, auf die "Trägheit und Bequemlichkeit vieler Bürger" zurück. „Es ist eben nicht so einfach wie beim Telefonieren, wo ich nur eine andere Vorwahl wählen muss, um einen günstigeren Tarif zu bekommen."

$[\ldots]$

Der folgende Textausschnitt stammt aus einem Kommentar von Bernward Janzing mit dem Titel "Der faule Verbraucher"“. Der Kommentar wurde in der taz Nr. 7252 vom 8. Januar 2004 auf Seite 11 abgedruckt. Er wird im Internet sowohl bei der Verbraucherzen- 
trale Hamburg (http://www.vzhh.de/ als auch beim Bund der Energieverbraucher e.V. (http://www.energienetz.de/) gehostet (jeweils abgerufen am 10. Januar 2005).

$[\ldots]$

[..] reden wir heute einmal über die Stromkunden. Über die privaten Verbraucher. Über diejenigen, die in Umfragen immer ihre Vorliebe für die erneuerbaren Energien bekunden, die zugleich aber zu träge sind, ihren Atomstrom zu kündigen. Und auch über diejenigen, die sich - gerade zum Jahreswechsel wieder - über steigende Strompreise ihres Exmonopolisten beklagen, es aber gleichwohl nicht schaffen, zu einem anderen Anbieter zu wechseln.

In Zahlen: Wir reden über 96 Prozent der deutschen Bevölkerung. Denn fünfeinhalb Jahre nach der Liberalisierung des Strommarktes machen erst 4 Prozent der Haushalte von der Freiheit Gebrauch, sich den Produzenten ihres Stroms selbst auszusuchen. Und die Zahl stagniert. Anders ausgedrückt: Unter 25 privaten Stromkunden ist statistisch nur einer, der sich bisher aufraffen konnte, seinen Strom dort einzukaufen, wo er ökologischer erzeugt und/oder billiger angeboten wird als beim angestammten Exmonopolisten.

Die Konsequenzen dieser Trägheit werden zunehmend deutlich: Die Privatkunden werden von den alteingesessenen Anbietern als mündige Marktteilnehmer kaum noch ernst genommen. Fast ist man wieder in jener Welt angelangt, in der Stromkunden noch Abnehmer hießen; in der die Monopolisten gegenüber den Verbrauchern behördengleich auftraten und Stromrechnungen verschickten im Outfit von Steuerbescheiden.

Die Verbraucher haben es sich selbst zuzuschreiben. Denn in einem Markt, dem sich 96 Prozent der Kunden verweigern, kann unmöglich ein Wettbewerb entstehen. Und das nutzen - was nur logisch ist - die alteingesessenen Anbieter aus. Sie erhöhen die Preise, weil sie wissen: Kaum ein Kunde wird deswegen zu einem Mitbewerber wechseln. Unbekümmert bieten sie zudem ihren Dreckmix von Atom- und Kohlestrom an, weil sie gleichermaßen darauf zählen können, dass die Bürger viel zu träge sind, sich eine Alternative zu suchen. $[\ldots]$

Der Wechsel des Stromanbieters ist inzwischen zu einer durchaus durchschaubaren Angelegenheit geworden. Man trägt seine Adresse in ein Formular ein, gibt seine Kontonummer an und schickt das Ganze unterschrieben mit einer Kopie der letzten Stromrechnung an den Lieferanten seiner Wahl. Mehr will der gar nicht wissen - und regelt dann den Rest. Wo also ist das Problem? Es liegt einzig und allein in den Köpfen und heißt schlicht Desinteresse. Denn der Strom ist so selbstverständlich, dass kaum jemand über ihn nachdenkt. Er ist einfach da und kommt aus der Steckdose.

Er ist, wie es im Marketingdeutsch so schön heißt, ein „Low-Interest-Produkt“. Und zwar, weil er ein „Low-Cost-Produkt" ist. Denn Strom war in Deutschland nie so günstig zu 
haben wie in den vergangenen Jahren. Daran ändert auch der jüngste Preisanstieg nichts. Zweiflern sei gesagt: Gemessen am Einkommen war der Haushaltsstrom in Deutschland vor zwanzig Jahren noch doppelt so teuer wie heute. In den Sechzigerjahren kostete er sogar noch das Vierfache.

$[\ldots]$ 